JOINT TRANSPORTATION RESEARCH PROGRAM

FHWA/IN/JTRP-2010/29

Final Report

PORTLAND CEMENT CONCRETE PAVEMENT PERMEABILITY PERFORMANCE

Javier Castro

Robert Spragg

Phil Compare

William Jason Weiss

November 2010 


\section{TECHNICAL Summary}

INDOT Research

Technology Transfer and Project Implementation Information

TRB Subject Code:

Publication No.:FHWA/IN/JTRP-2010/29, SPR-3093

November 2010

Final Report

\section{Portland Cement Concrete Pavement Permeability Performance}

\section{Introduction}

The main objective of this study was to evaluate the fluid transport properties of concrete pavements constructed in the state of Indiana.

The scope of the research included characterization of fluid transport using two primary tests that included water absorption and electrical conductivity.

Specifically this work evaluated the absorption of water and/or deicing solutions, and electrical conductivity. A series of concrete paving mixtures were tested to provide a range of values that were typical for the state of Indiana. While similar mixture proportions were used for the mixtures in Indiana differences in the magnitude of water absorbed occurred. A series of mortars were tested to illustrate the effect of curing conditions, water to cement ratio, and paste volume.

In addition to the measurement of transport properties, the relative humidity was assessed for concrete slabs exposed to different exposure conditions. The samples considered in this investigation included a sample stored at $50 \%$ relative humidity, covered concrete, a concrete with an exposed vertical surface, a concrete on a drainable base, a concrete on a non-drainable base, and concrete that was submerged.

\section{Findings}

The following conclusions can be drawn :

- While similar mixture proportions were used for paving mixtures in Indiana differences in the magnitude of water absorbed occurred.

- Samples dried to a lower relative humidity showed a greater volume of water absorbed.

- Drying water absorption samples at $105 \mathrm{C}$ resulted in substantial anomalies in water absorption, as such this method is not recommended.

- Absorption samples should account for the volume of paste in the sample when this varies

- $\quad$ Samples were tested using deicing solutions as the absorbing fluid. In addition, samples were tested that had previously been exposed to deicing solutions. In both cases the presence of salts altered the viscosity and

surface tension resulting in differences in the water absorption.

- The influence water addition to a concrete mixture was able to be determined using electrical conductivity.

- Pore solution conductivity was observed to be approximately linearly related to the degree of hydration.

- A correction must be applied to electrical conductivity or resistivity samples tested at different temperatures.

- Practical field samples the relative humidity in the concrete was always above $80 \%$ for the fall winter and spring. The samples that were exposed to precipitation events demonstrated higher relative humidities. 


\section{Implementation}

The results of this investigation indicate that fluid transport can vary significantly even when the similar mixture proportions are used. This work will be combined with results of a national pooled fund project that can promote the development of a new testing procedure that can rapidly assess the fluid transport properties of concrete. This work has laid the foundation for using water absorption and electrical conductivity.

The results demonstrate the importance of the fluid being absorbed as well as the sample conditioning in obtaining meaningful results. It was demonstrated that even when samples are previously exposed to deicing salts which can alter the rate of fluid absorption. This needs to be considered in the evaluation of field samples.

Tests for rapid conditioning of absorption samples are being evaluated as part of the pooled fund study and will be used as a rapid test is developed. Similarly, INDOT is currently involved in the evaluation of electrical conductivity testing for the potential development of a new standard testing procedure. The results of this work can be combined with the results of that study for the development of a new testing procedure to rapidly assess the quality of concrete.

\section{Contact}

For more information:

\section{Prof. Jason Weiss}

Principal Investigator

School of Civil Engineering

Purdue University

West Lafayette, IN 47907

Phone: (765) 494-2215

Fax: (765) 496-1364
Indiana Department of Transportation

Division of Research

1205 Montgomery Street

P.O. Box 2279

West Lafayette, IN 47906

Phone: (765) 463-1521

Fax: (765) 497-1665

\section{Purdue University}

Joint Transportation Research Program

School of Civil Engineering

West Lafayette, IN 47907-1284

Phone: (765) 494-9310

Fax: (765) 496-1105 
Final Report

FHWA/IN/JTRP-2010/29

\title{
Portland Cement Concrete Pavement Permeability Performance
}

\author{
By \\ Javier Castro \\ Graduate Research Assistant \\ Robert Spragg \\ Undergraduate Research Assistant \\ Phil Kompare \\ Graduate Research Assistant \\ William Jason Weiss \\ Principal Investigator \\ School of Civil Engineering \\ Purdue University \\ Joint Transportation Research Program \\ Project Number: C-36-61R \\ File Number: 05-14-18
}

SPR-3093

Prepared in Cooperation with the Indiana Department of Transportation

The contents of this report reflect the views of the authors, who are responsible for the facts and the accuracy of the data presented herein. The contents do not necessarily reflect the official views and policies of the Indiana Department of Transportation. The report does not constitute a standard, specification or regulation. 


\begin{tabular}{|c|c|c|}
\hline $\begin{array}{l}\text { 1. Report No. } \\
\text { FHWA/IN/JTRP-2010/29 }\end{array}$ & 2. Government Accession No. & 3. Recipient's Catalog No. \\
\hline \multicolumn{2}{|c|}{$\begin{array}{l}\text { 4. Title and Subtitle } \\
\text { Portland Cement Concrete Pavement Permeability Performance }\end{array}$} & $\begin{array}{l}\text { 5. } \quad \text { Report Date } \\
\text { November } 2010\end{array}$ \\
\hline \multicolumn{2}{|c|}{$\begin{array}{l}\text { 7. Author(s) } \\
\text { Javier Castro, Robert Spragg, Phil Kompare and W. Jason Weiss }\end{array}$} & $\begin{array}{l}\text { 8. Performing Organization Report No. } \\
\text { FHWA/IN/JTRP-2010/29 }\end{array}$ \\
\hline \multirow{2}{*}{\multicolumn{2}{|c|}{$\begin{array}{l}\text { 9. Performing Organization Name and Address } \\
\text { Joint Transportation Research Program } \\
\text { Purdue University } \\
550 \text { Stadium Mall Drive } \\
\text { West Lafayette, IN 47907-2051 }\end{array}$}} & 10. Work Unit No. \\
\hline & & $\begin{array}{l}\text { 11. Contract or Grant No. } \\
\text { SPR-3093 }\end{array}$ \\
\hline \multirow{2}{*}{\multicolumn{2}{|c|}{$\begin{array}{l}\text { 12. Sponsoring Agency Name and Address } \\
\text { Indiana Department of Transportation } \\
\text { State Office Building } \\
100 \text { North Senate Avenue } \\
\text { Indianapolis, IN } 46204\end{array}$}} & $\begin{array}{c}\text { 13. Type of Report and Period Covered } \\
\text { Final Report }\end{array}$ \\
\hline & & 14. Sponsoring Agency Code \\
\hline \multicolumn{3}{|l|}{ 15. Supplementary Notes } \\
\hline
\end{tabular}

\section{Abstract}

The objective of this project was to evaluate the transport properties of concrete pavement in the state of Indiana using common testing procedures. Specifically this work evaluated the absorption of water, the absorption of deicing solutions, and electrical conductivity. A series of concrete paving mixtures were tested to provide a range of values that were typical for the state of Indiana. While similar mixture proportions were used for the mixtures in Indiana differences in the magnitude of water absorbed occurred. A series of mortars were tested to illustrate the effect of curing conditions, water to cement ratio, and paste volume. It was observed that a long duration of drying was needed to obtain equilibrium. Samples dried to a lower relative humidity showed a greater volume of water absorbed. It was observed that drying at $105 \mathrm{C}$ resulted in substantial anomalies in water absorption, as such this method is not recommended. It was observed that when samples were tested using deicing solutions or samples were tested that were previously exposed to deicing solutions the water absorption could be influenced. The electrical conductivity work was performed as a potential method to develop the understanding of rapid test techniques for quality control. The research used a modified parallel law to relate the electrical conductivity to the pore volume, pore solution conductivity and the tortuosity through the pore network. The influence water addition was able to be determined using electrical conductivity. In addition, the pore solution was observed to be approximately linearly related to the degree of hydration. It is critical that a correction be applied to samples tested at different temperatures. An activation energy of conduction was observed that was approximately $10 \mathrm{~kL} / \mathrm{mol}$ irrespective of water to cement ratio. In addition to the measurement of transport properties, the relative humidity was assessed for concrete exposed to different exposure conditions. The samples considered in this investigation included a sample stored at $50 \%$ relative humidity, covered concrete, a concrete with an exposed vertical surface, a concrete on a drainable base, a concrete on a non-drainable base, and concrete that was submerged. The samples showed that for practical field samples the relative humidity in the concrete was always above $80 \%$ for the samples tested. The samples that were exposed to precipitation events demonstrated higher relative humidities.

\begin{tabular}{|c|c|c|c|c|}
\hline \multicolumn{2}{|c|}{$\begin{array}{l}\text { 17. Key Words } \\
\text { Absorption, concrete, deicer solutions, electrical resistivity, } \\
\text { electrical conductivity, permeability, relative humidity, } \\
\text { transport }\end{array}$} & \multicolumn{3}{|c|}{$\begin{array}{l}\text { 18. Distribution Statement } \\
\text { No restrictions. This document is available to the public through the } \\
\text { National Technical Information Service, Springfield, VA } 22161\end{array}$} \\
\hline 19. Security Classif. (of this report) & \multicolumn{2}{|c|}{ 20. Security Classif. (of this page) } & 21. No. of Pag & 22. Price \\
\hline Unclassified & \multicolumn{2}{|c|}{ Unclassified } & 249 & \\
\hline
\end{tabular}


2

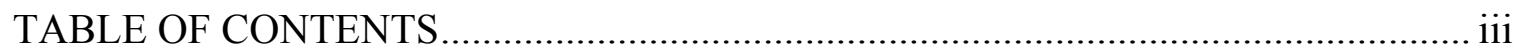

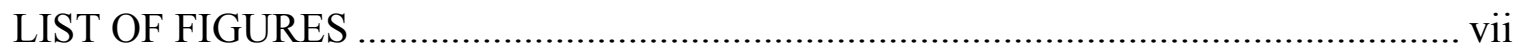

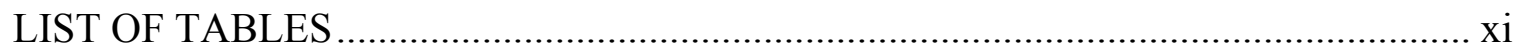

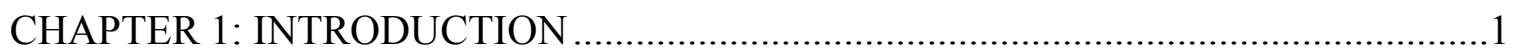

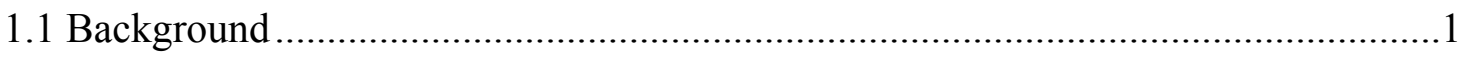

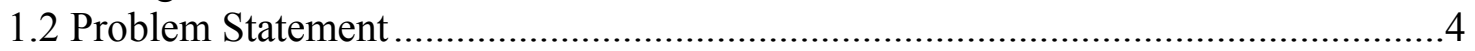

1.3 Research Objective and Scope of Project ...........................................................

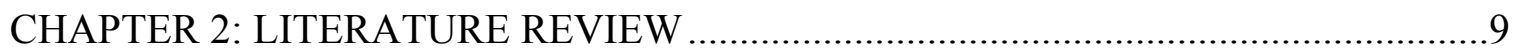

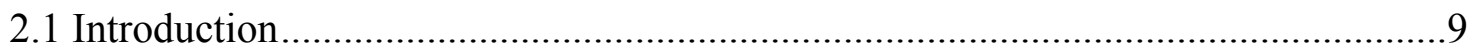

2.2 Review of Theory of Fluid Transport in Concrete................................................

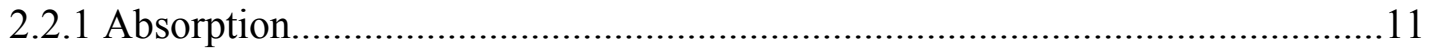

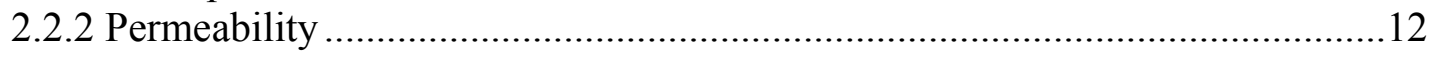

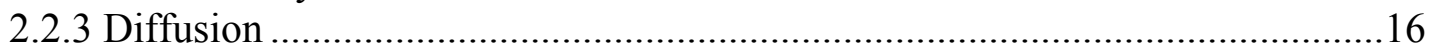

2.2.4 Effect of Mixture Proportions and Curing on Transport Properties ..................19

2.2.5 Effect of Samples Preparation on Tests Results ................................................22

2.3 Methods to Measure Gas and Water Permeability in Concrete................................25

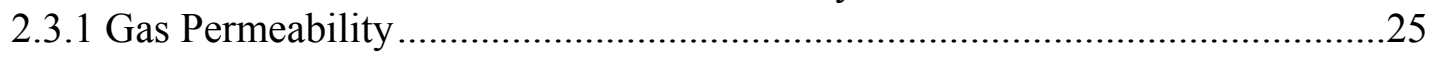

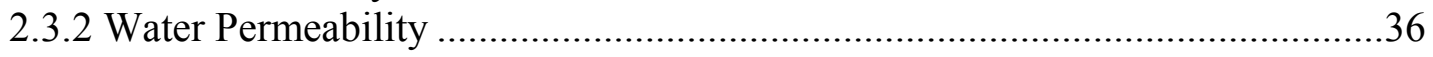

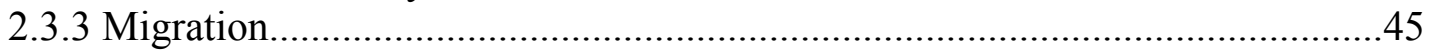

2.4 Summary and Conclusions ……………………….........................................56

CHAPTER 3: TRANSPORT PROPERTIES OF SAMPLES OBTAINED FROM THE

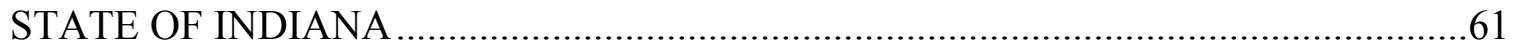

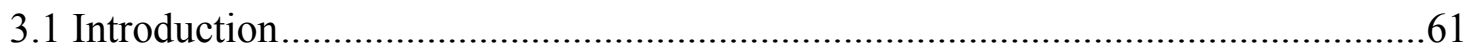

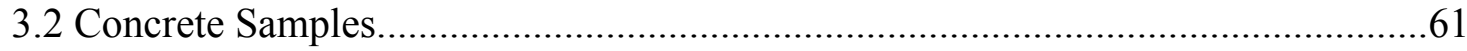

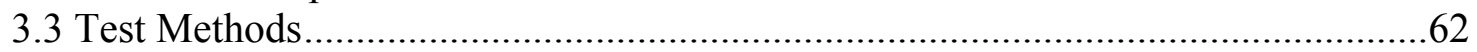

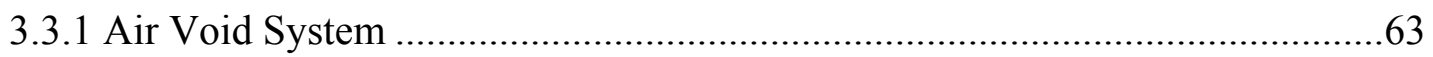

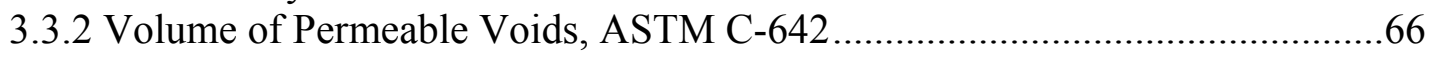

3.3.3 Water Absorption of the Concrete, ASTM C-1585 ………………..................68

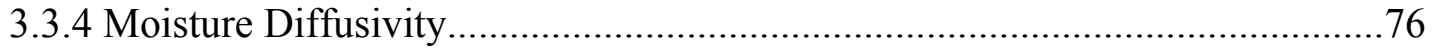

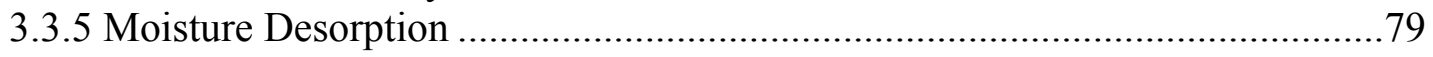

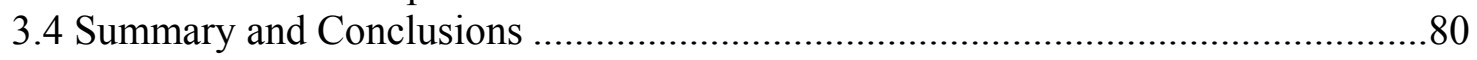




\section{CHAPTER 4: EFFECT OF SAMPLE CONDITIONING ON THE WATER}

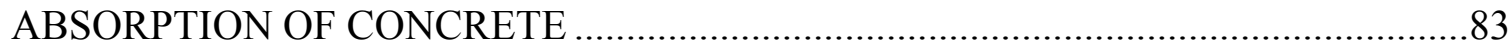

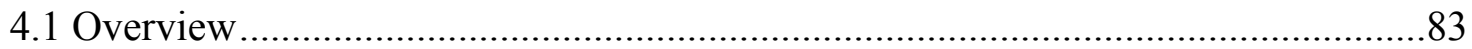

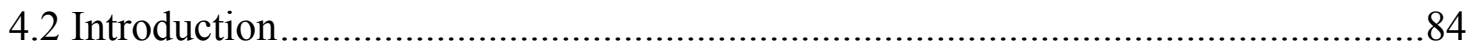

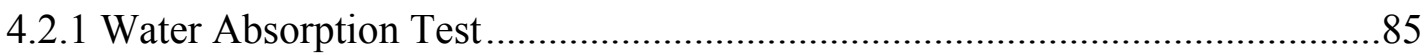

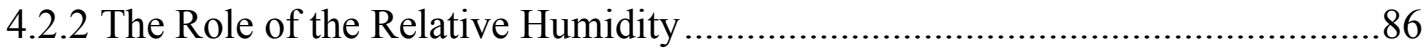

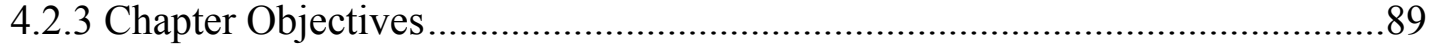

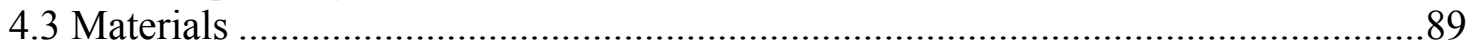

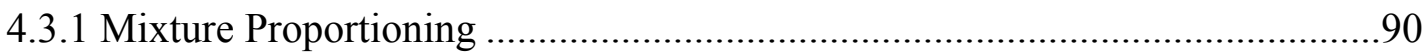

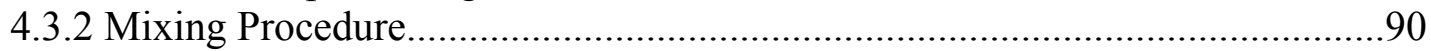

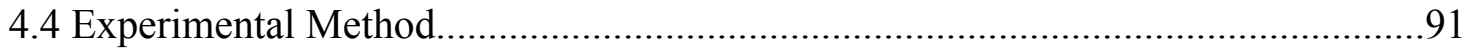

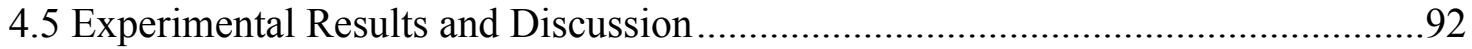

4.5.1 Desorption Isotherms ............................................................................92

4.5.2 Effect of Initial Conditioning on Water Absorption Tests................................93

4.5.3 Effects of Relative Humidity on the Amount of Absorbed Water after 8

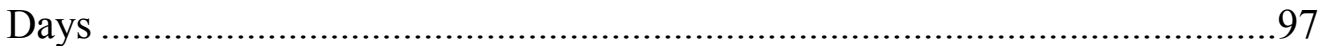

4.5.4 Effects of Relative Humidity on Initial Sorptivity ............................................99

4.5.5 Effects of Relative Humidity on Secondary Sorptivity ...................................100

4.6 Effects of Initial Moisture of Samples on ASTM C1585 Conditioning Method...101

4.7 Effects of Volume of Aggregate on Sorption Test .................................................102

4.8 Summary and Conclusions ...............................................................................106

\section{CHAPTER 5: WETTING AND DRYING OF CONCRETE IN THE PRESENCE OF}

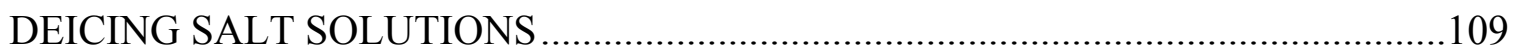

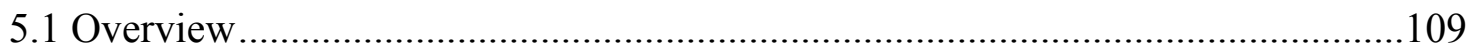

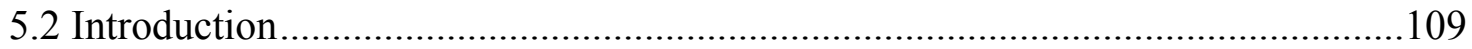

5.3 Fluid Absorption in Porous Materials............................................................110

5.4 Wetting and Drying for Concrete with Deicing Solutions.....................................113

5.4.1 Experimental Program of Wetting and Drying of Concrete with Deicing

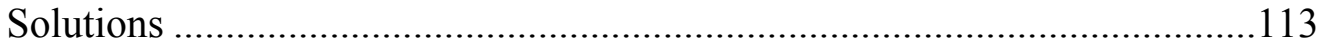

5.4.2 Experimental Results from Wetting with Different Conditioning Methods...115

5.4.3 Experimental Results from Wetting and Drying with Deicing Solutions.......116

5.4.4 Experimental Results from Wetting Previously Exposed to Deicing

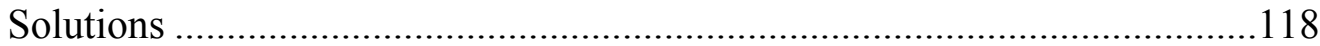

5.4.5 Drying of Mortars Saturated with Different Deicing Salts .............................120

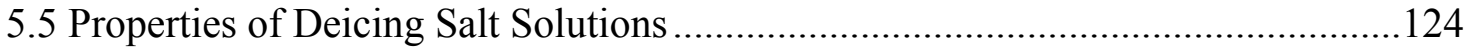

5.5.1 Surface Tension of Deicing Salt Solutions ....................................................124

5.5.2 Viscosity of Deicing Salt Solutions .............................................................125

5.5.3 Relative Humidity of Deicing Salt Solutions.................................................127

5.5.4 Specific Gravity of Deicing Salt Solutions ...................................................127

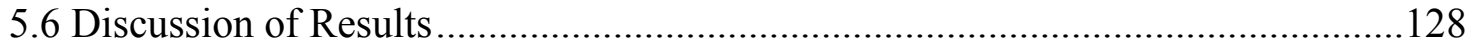

5.6.1 Aqueous Solution Absorption Behavior as a Function of Surface Tension

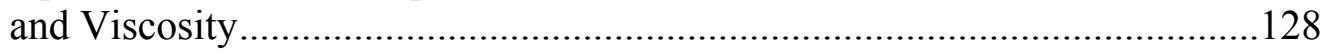

5.6.2 Drying Time Versus Wetting Time …………............................................129 


\section{CHAPTER 6: SORPTION TESTING IN CEMENTITIOUS MATERIALS: A} DISCUSSION OF AUTOMATING TEST PROCEDURES .

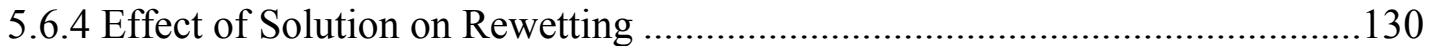

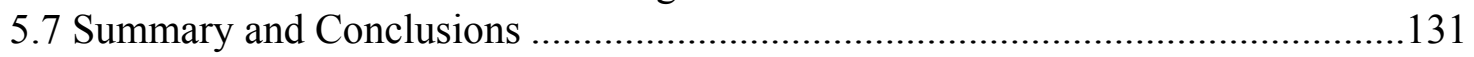

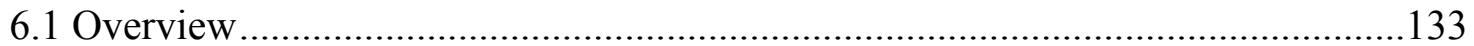

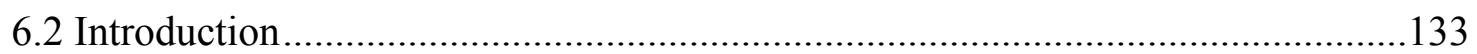

6.3 Standard ASTM C-1585 Procedure ...................................................................135

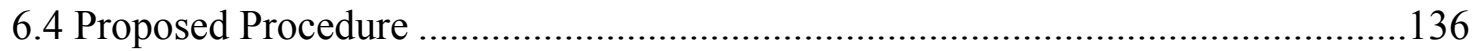

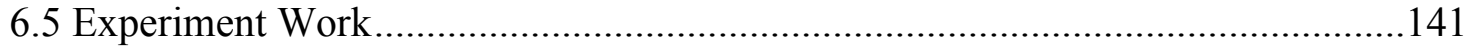

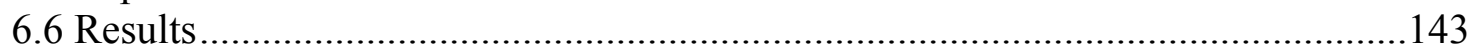

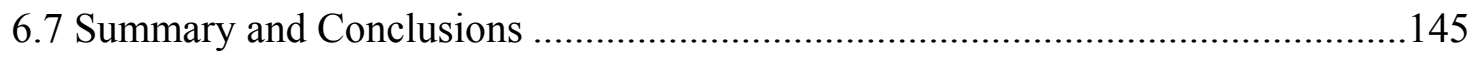

CHAPTER 7: ELECTRICAL IMPEDANCE SPECTROSCOPY AND RELATED

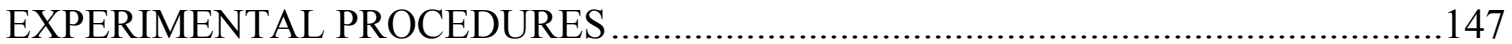

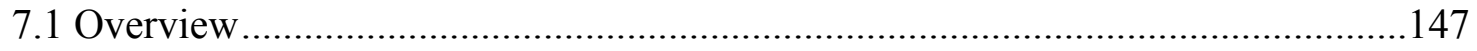

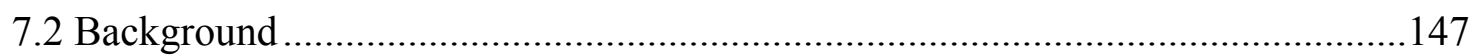

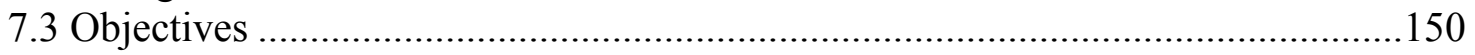

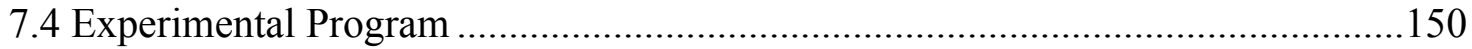

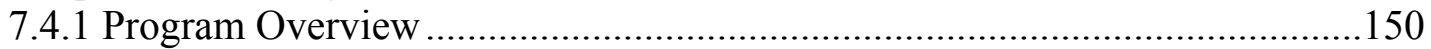

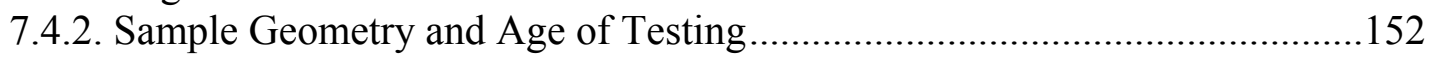

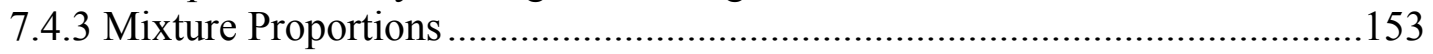

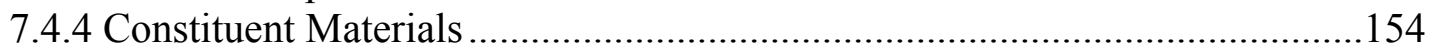

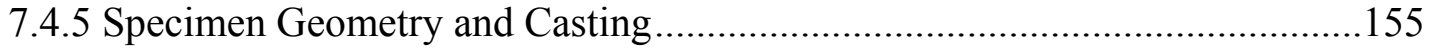

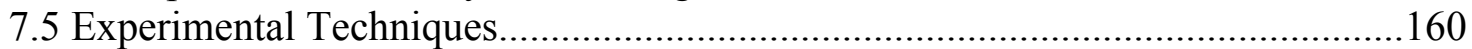

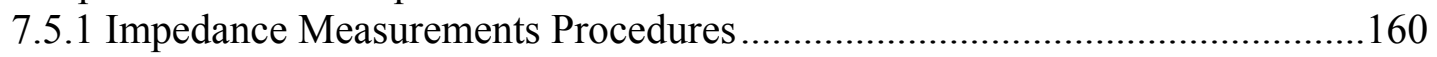

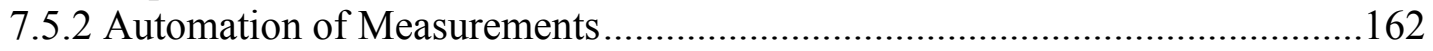

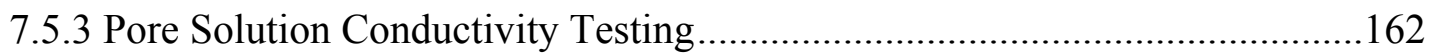

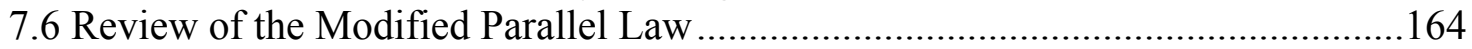

7.6.1 Powers Model for Computation for Hydration.............................................166

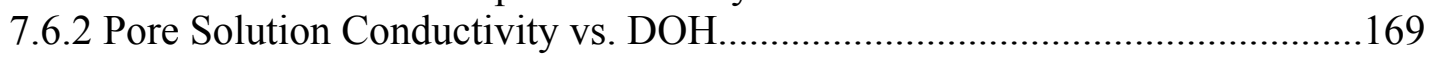

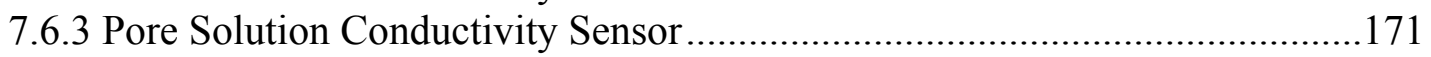

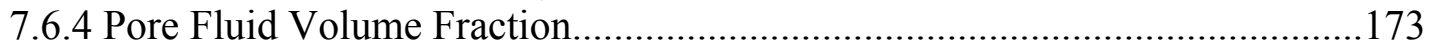

7.6.5 Effect of Temperature on Pore Solution and Concrete Conductivity .............175

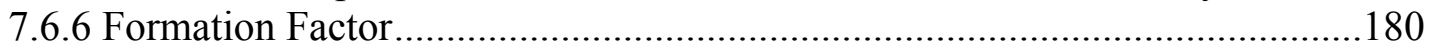

7.7 The Effect of Mixture Design on the Conductivity of Concrete ..........................182

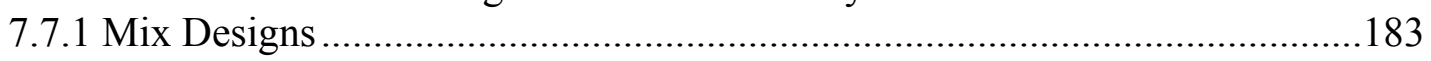

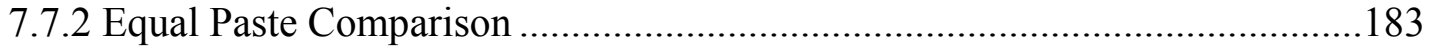

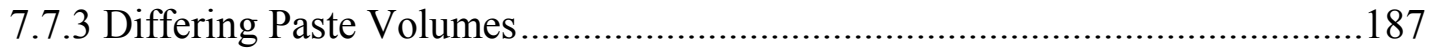

7.7.4 Increased Water Content............................................................................ 190

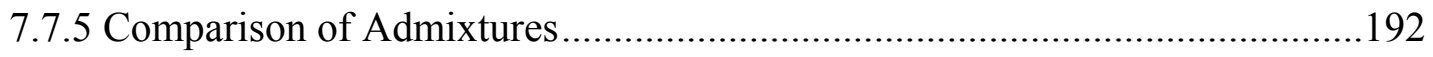

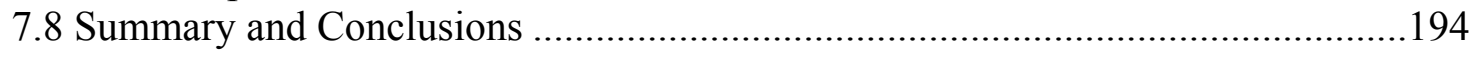




\section{CHAPTER 8: MONITORING INTERNAL RELATIVE HUMIDITY IN EXPOSED} CONCRETE SLABS WITH VARIOUS EXPOSURE CONDITIONS........................197

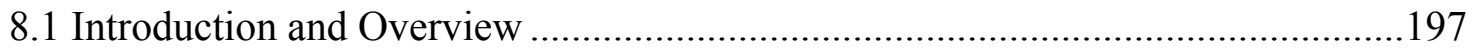

$4 \quad 8.2$ Boundary Conditions ............................................................................... 198

$5 \quad 8.3$ Evaluation of the Relative Humidity Sensors .................................................206

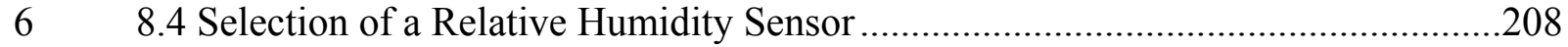

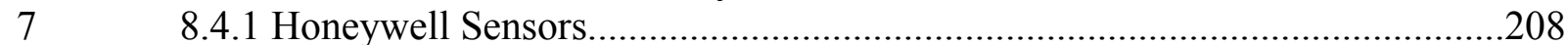

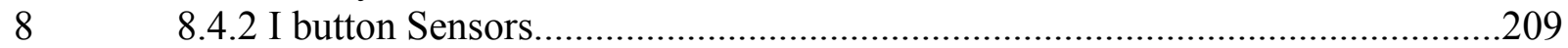

98.5 Design of the Slab Specimens....................................................................2 212

$10 \quad 8.6$ Design of the Relative Humidity Sensor Connection .......................................217

118.7 Placement of the Relative Humidity Sensor in the Cylindrical Hole ...................218

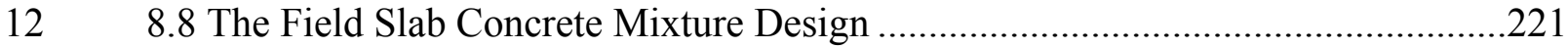

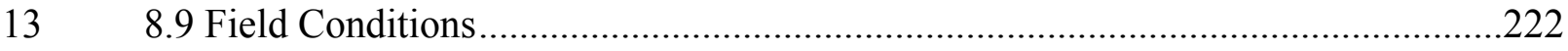

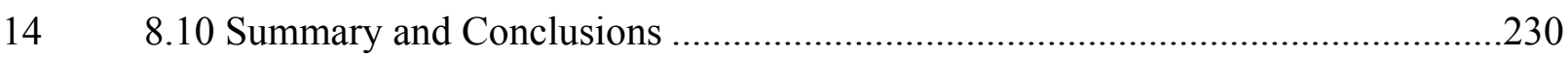

CHAPTER 9: SUMMARY, CONCLUSIONS AND RECOMMENDATIONS ............231

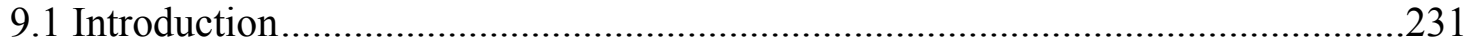

189.2 Summary and Conclusions from Experimental Studies ..................................231

19 9.2.1 Transport Properties on Concrete Pavement from the State of Indiana.........231

$20 \quad 9.2 .2$ Water absorption on Mortar Samples Cast on Laboratory ...........................232

219.2 .3 Fluid Absorption on Concrete Samples Using Deicing Solutions.................233

229.2 .4 Automated Water Absorption Test ......................................................234

$23 \quad 9.2 .5$ Electrical Conductivity on Concrete .....................................................234

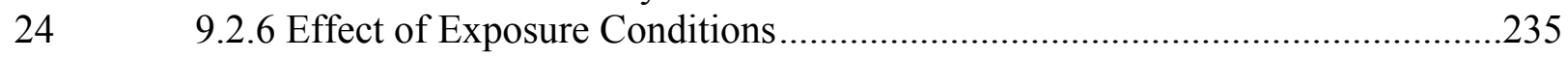

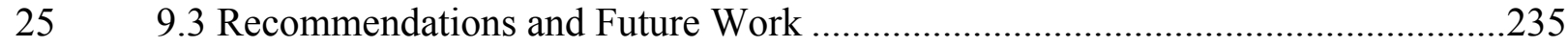




\section{LIST OF FIGURES}

Figure 1.1: An Example of the Relationship Between the Permeability Properties of a Concrete to its Service Life when Exposed to Freezing and Thawing Cycles.............................................................................................

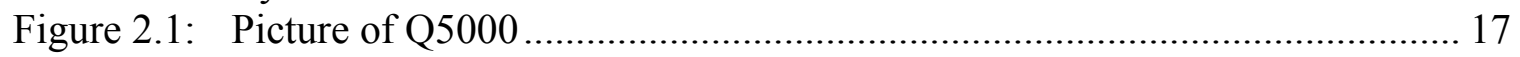

Figure 2.2: A Typical Mass Change versus Time Plot for LWA prewetted with $1 \mathrm{~h}$ Synthetic Pore Solution as Example of the Technique............................. 18

Figure 2.3: Schematic View of the Sch nlin Method ................................................ 26

Figure 2.4: Schematic of Surface Airflow Test .......................................................... 28

Figure 2.5: Autoclam Apparatus for Permeability Tests............................................ 29

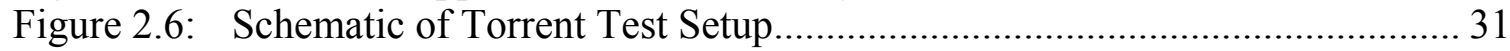

Figure 2.7: Schematic of the TUD Method ................................................................ 33

Figure 2.8: Schematic View of Hong-Parrott Method ............................................... 34

Figure 2.9: Schematic View of the Germann Gas Permeation Test Method ................. 35

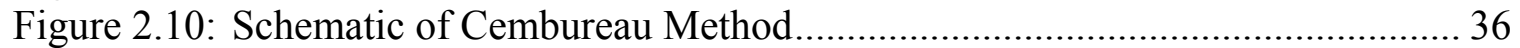

Figure 2.11: Schematic View of the ISAT Method....................................................... 38

Figure 2.12: Schematic Setup of Autoclam Test for Water Permeability Measurement . 40

Figure 2.13: Schematic Illustration of the GWT Setup............................................ 41

Figure 2.14: Schematic Illustration of the Figg-Poroscope Method ............................. 43

Figure 2.15: Schematic View of the Florida Test Setup .............................................. 44

Figure 2.16: Schematic Illustration of the 4 Point (Wenner) Method for Resistivity Measurement.................................................................................... 46

Figure 2.17: Test Setup of the Galvanostatic Pulse Technique..................................... 47

Figure 2.18: Potential Response of the Galvanostatic Pulse Technique ......................... 48

Figure 2.19: Nyquist Plot Obtained from and EIS Test .............................................. 49

Figure 2.20: Schematic of Rapid Chloride Permeability Test....................................... 50

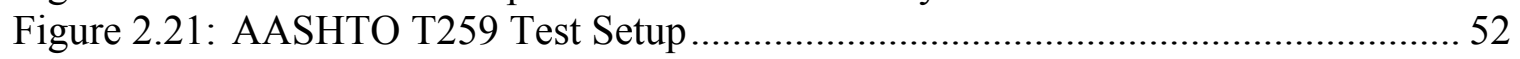

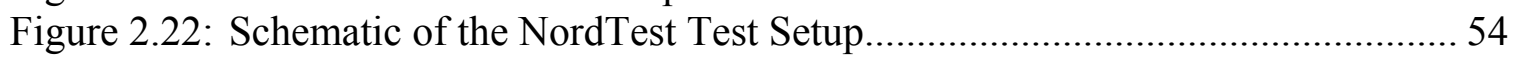

Figure 2.23: Tang and Nilsson Migration Cell............................................................ 55

Figure 3.1: Prepared Samples for Air Void Count, Automated Method ...................... 65

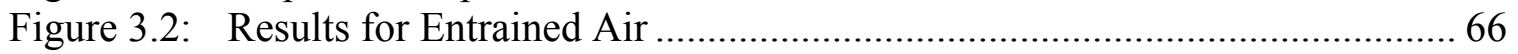

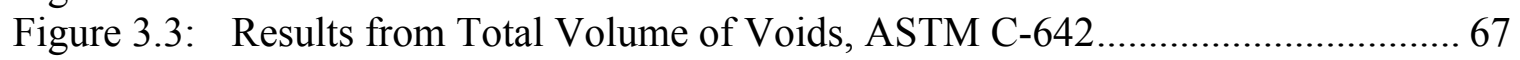

Figure 3.4: Water Absorption Test, Concretes \#1 and \#2: Plain Cement with Standard Aggregate........................................................................ 71

Figure 3.5: Water Absorption Test, Concretes \#3 and \#4: Plain Cement with Slag Aggregate ................................................................................. 71

Figure 3.6: Water Absorption Test, Concretes \#5 to \#9: Cement plus Fly Ash with Standard Aggregate ...................................................................... 72

Figure 3.7: Water Absorption Test, Concretes \#10 and \#11 Cement plus Fly Ash with Slag Aggregate............................................................................ 73

Figure 3.8: Water Absorption Test, Concretes \#12 and \#13 Cement plus Fly Ash and Silica Fume with Standard Aggregate.............................................. 73

Figure 3.9: Water Absorption Test, Concretes \#14, Cement plus Slag with Slag Aggregate. 
1 Figure 3.10: Normalized Mass Loss of Cement Paste Sample at Different RH .............. 77

2 Figure 3.11: Diffusivity of Cement Paste Sample as a Function of RH........................... 78

3 Figure 3.12: Desorption Curve on 18 Months Old Mortar Sample................................... 80

4 Figure 4.1: Relation Between Relative Humidity and Partially Empty Pores in

Figure 4.2: Desorption Curve on 18 Months Old Mortar Samples

Figure 4.3: Absorbed Water in Mortars as a Function of Relative Humidity: a) Mixture 55/0.35, b) Mixture 55/0.40, c) Mixture 55/0.45, d) Mixture $55 / 0.50$

Figure 4.4: Degree of Saturation as a Function of Time During the Water Absorption Test: a) Mixture 55/0.35, b) Mixture 55/0.40, c) Mixture $55 / 0.45$, d) Mixture 55/0.50.

Figure 4.5: Degree of Saturation at 90 Days in Contact with Water as a Function of the $\mathrm{w} / \mathrm{c}$

Figure 4.6: Cumulative Absorption at 8 Days for Mortars Versus: a) w/c, b) Relative

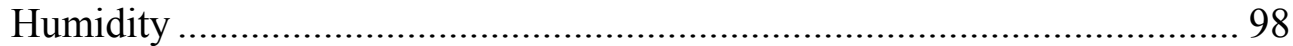

Figure 4.7: Cumulative Absorption at 8 Days Versus w/c and Relative Humidity: a) Normalized to Absorption of Mixture 55/0.35, b) Normalized to Absorption at $50 \% \mathrm{RH}$

Figure 4.8: Initial Absorption on Mortars Conditioned at Different RH Function of:

a) w/c, b) Relative Humidity.

Figure 4.9: Secondary Absorption on Mortars Conditioned at Different RH Function of: a) w/c, b) Relative humidity. Solid Lines are Provided to Show a General Tendency in the Data.

Figure 4.10: Effect of Initial Moisture on the Conditioned Procedure Established in ASTM C1585-04: a) Mixture 55/0.35, b) Mixture 55/0.40, c) Mixture 55/0.45, d) Mixture 55/0.50

Figure 4.11: Initial and Secondary Sorptivity on Mortars with Different Initial Moisture Content, Conditioned with the Procedure Established in ASTM C1585-04

Figure 4.12: Water Absorption in Mortars Containing Different Volume of Aggregates: a) Normalized by Surface in Contact with Water, (b) Normalized by Volume of Paste.....

Figure 4.13: Water Absorption at 8 Days Normalized by Volume of Paste, Corrected by Different Values of Aggregate Absorption............................................. 105

Figure 4.14: Desorption Isotherm for the Sand used in this Study ............................... 106

Figure 5.1: Water Absorption on Samples Subjected to Different Conditioning

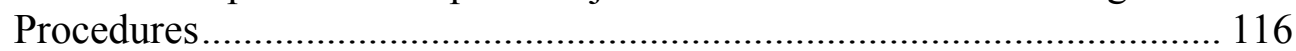

Figure 5.2: Volume of Deicing Solutions Absorbed by Concrete as a Function of

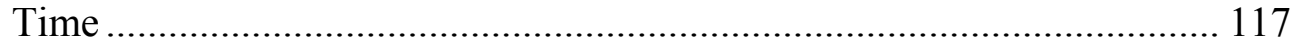

Figure 5.3: Drying of Concrete Prewetted with Different Salt Solutions as a Function of Time.

Figure 5.4: Volume of De-ionized Water Absorbed by Concrete as a Function of Time in the Second Fluid Absorption Test

Figure 5.5: Change at Decreasing RH for Samples Containing De-ionized Water ..... 121 
1 Figure 5.6: Mass Change for Samples Submerged in Aqueous Solutions Containing

Deicing Salts: (a) $\mathrm{NaCl} 23 \%$ (b) $\mathrm{CaCl} 232 \%$ and (c) $\mathrm{MgCl} 230 \%$ 123

Figure 5.7: Properties of Deicing Salts at $23-25^{\circ} \mathrm{C}$ : (a) Surface Tension (b) Viscosity (c) Relative Humidty (d) Specific Gravity ............................. 126

Figure 5.8: Relative Sorptivity for Deicing Solutions............................................. 129

Figure 6.1: Automated Test Procedure: a) Photo of an Actual Test, and b)

Representation of Specimen in Support Device 137

Figure 6.2: Schematic Representation of Setup of Proposed Procedure 139

Figure 6.3: Total Water Loss During a Test of an Impermeable Plastic Sample and Total Evaporated Water from Reservoir of Water Covered with Paraffin Oil

Figure 6.4: Preparation of Specimen for Automates Procedure a) Representation of Sample Preparation b) Photo of the Sample Ready to be Tested .............. 143

Figure 6.5: Comparison of Absorbed Water of Samples Conditioned at 50\%RH: a) vs time, b) vs Square Root of Time 144

Figure 6.6: Comparison of Absorbed Water of Samples Conditioned at 65\%RH: a) vs. Time, b) vs. Square Root of Time .................................................... 144

Figure 7.1: Nyquist Plot and Bode Plot ................................................................... 149

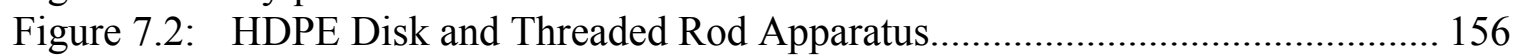

Figure 7.3: Schematic of Mold Design.................................................................... 157

Figure 7.4: Finished Specimen with Rods and Lid in Place...................................... 158

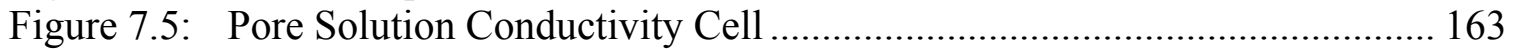

Figure 7.6: Geometry Corrected EIS Conductivity Data .......................................... 164

Figure 7.7: Total Energy Evolved from Calorimeter .............................................. 167

Figure 7.8: Degree of Hydration for 0.45 Paste .......................................................... 168

Figure 7.9: 0.45 w/c Pore Solution Conductivity vs. Time ........................................ 170

Figure 7.10: Pore Solution Conductivity vs. DOH for a Paste with a w/c of $0.45 \ldots \ldots \ldots 170$

Figure 7.11: Front and Side View of the Rajabipour Developed Pore Solution Sensor 172

Figure 7.12: Sensor Conductivity vs. Pore Solution Conductivity ............................. 173

Figure 7.13 Pore Fluid Fraction vs. Degree of Hydration for 0.42 w/c Paste.............. 174

Figure 7.14 Pore Fluid Volume Fraction vs. DOH for a 65.7\% Agg. Fraction 0.42 w/c Concrete .................................................................................... 175

Figure 7.15 Natural Log of Pore Solution Conductiviy vs. the Inverse of the

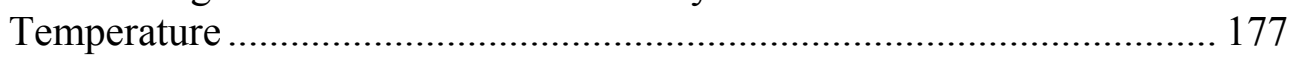

Figure 7.16 0.45 w/c 27.7\% Raw EIS Data............................................................. 179

Figure 7.170 .45 w/c Concrete (27.7\% Paste Volume) - Early Age Data ................... 179

Figure 7.180 .45 w/c (27.7\% Paste Volume) - Time and Temperature Corrected Conductivity Data - Early Age ....................................................... 180

Figure 7.19 Formation Factor vs. Degree of Hydration for a 0.45 w/c 27.7\% Paste Volume Concrete ................................................................................ 181

Figure 7.20: Beta Factor vs. Degree of Hydration for a 0.45 w/c 27.7\% Paste Volume Concrete.

Figure 7.21: Temperature Corrected Conductivity vs. Equivalent Time - Early Age... 184

Figure 7.22: Temperature Corrected Conductivity vs. Equivalent Time - Late Age .... 185

Figure 7.23: Equal Paste Comparison - Normalized Conductivity 186 
1 Figure 7.24: Equal Paste Comparsion - Beta Factor...

Figure 7.25: Conductivity vs. Degree of Hydration for Equal W/C Concretes with Differing Paste Volumes

Figure 7.26: Percent Greater Conductivity of the Increased Paste Mixture Compared to the Standard Mixture

Figure 7.27: Conductivity of the System / Pore Solution Conductivity vs. Degree of Hydration for Equal W/C Concretes with Differing Paste Volumes

Figure 7.28: Connectivity Factor vs. Degree of Hydration Comparison of Equal w/c Concretes with Different Paste Volumes.

Figure 7.29: Temperature Corrected Conductivity vs. Degree of Hydration Over an Increase in Water Content

Figure 7.30: 1 / Formation Factor vs. Degree of Hydration Over an Increase in Water Content.

Figure 7.31: Connectivity Factor vs. Degree of Hydration over an Increase in Water Content

Figure 7.32: Comparison of Conductivity of Samples Containing Admixtures

Figure 7.33: Connectivity of the System vs. DOH for the Admixture Comparison Samples

Figure 7.34: Connectivity of the System vs. DOH for the Admixture Comparison Samples

Figure 8.1: Exposure Samples at the INDOT Research Test Facility

Figure 8.2: Weather Station at the INDOT Research Test Facility..... 200

Figure 8.3: Covered Sample at the INDOT Research Test .......................................... 201

Figure 8.4: Exposed Vertical Surface Sample at the INDOT Research Test Facility.. 202

Figure 8.5: A Horizontal Surface on a Drainable Base at the INDOT Research Test Facility

Figure 8.6: A Horizontal Surface on A Non-Drainable Base at the INDOT Research Test Facility .....

Figure 8.7: A Submerged Concrete Sample at the INDOT Research Test Facility ..... 205

Figure 8.8: A Sample Exposed to a Constant Drying Environment.............................. 206

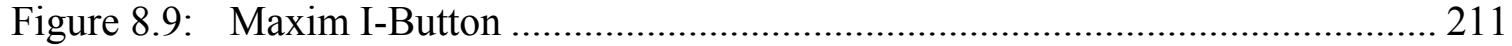

Figure 8.10: Test Slab Dimensions ......................................................................... 214

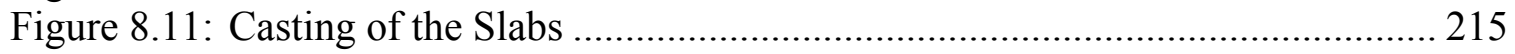

Figure 8.12: Removing PVC Pipes from Slab Using UTM ………………………... 216

Figure 8.13: I-button Telephone Line Setup ............................................................ 218

Figure 8.14: Temperature Response from the Weather Station s................................... 223

Figure 8.15: Rainfall Response from the Weather Station............................................ 223

Figure 8.16: Relative Humidity Response from the Weather Station ............................. 224

Figure 8.17: Wind Speed from the Weather Station .................................................... 224

Figure 8.18: Relative Humidity Measured in the Concrete in the $50 \%$ Environment .. 227

41 Figure 8.19: Relative Humidity Measured in the Concrete in the Covered Slab .......... 227

42 Figure 8.20: RH Measured in the Concrete in the Exposed Vertical Slab .................... 228

43 Figure 8.21: Relative Humidity in the Concrete in the Slab on a Drainable................. 228

44 Figure 8.22: Relative Humidity in the Concrete in the Slab on an Undrainable Base ... 229

45 Figure 8.23: Relative Humidity Measured in the Concrete in the Submerged Slab ...... 229 
2 Table 2.5: Summary of test methods described in this literature review ...................... 57

3 Table 3.1: Characteristics of Concrete Sample Collected from the State of Indiana....... 62

4 Table 4.1: Mixture Proportions in Saturated Surface Dry (SSD) Conditions................. 90

5 Table 5.1: Mixture Proportions Assuming Saturated Surface Dry (SSD) Conditions... 113

6 Table 6.1: Mixture Proportions, SSD Condition........................................................... 141

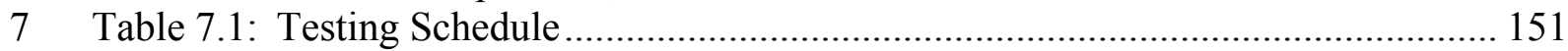

8 Table 7.2: Equal Paste Content Testing Breakdown............................................... 151

9 Table 7.3: Equal Water to Cement Ratio Testing Breakdown.................................... 152

10 Table 7.4: Increased Water Content Testing Breakdown .......................................... 152

11 Table 7.5: Increased Admixture Testing Breakdown ............................................. 152

12 Table 7.6: Mixture Proportion for Electrical conductivity Testing, in SSD Condition. 154

13 Table 7.7: Activation Energy of Conduction for Different w/c Mixtures .................... 177

14 Table 8.1: Salt Solution Calibration of Humidity Sensor \#28 .................................... 207

15 Table 8.2: Salt Solution Calibration of Humidity Sensor \#21 .................................... 207

16 Table 8.3: Field Slab Mixture Proportions by Mass in SSD Condition....................... 221

17 Table 8.4: Field Slab Mixture Proportions by Volume............................................... 222

18 Table 8.5: Recalibration of an I-button after the Test was Finished ............................ 225 


\section{$\underline{1.1 \text { Background }}$}

3 Several recent studies have indicated the importance of fluid transport on the

4 durability of concrete pavements. For example, a recent survey by the Iowa Department

5 of Transportation (Nantung and Byers 2006) indicated a correlation between permeability

6 of a concrete pavement and its durability. The Iowa Department of Transportation

7 measured the permeability of cores taken from concrete pavements that have been in

8 service for over eighty years. The results of these scores showed that the low

9 permeability of this concrete aides its ability to withstand environmental loading. Similar

10 anecdotal observations have been made in Michigan on sections of I-94 outside of Detroit

11 that are performing well after fifty years of service (Nantung 2006). Indiana has similar

12 experience with their concrete pavements. For example Yang et al. (2005) demonstrated

13 a relationship between rate of water absorption and the level of damage that exists in

14 concrete pavements. Cores from around the state of Indiana demonstrated that

15 pavements with high rate of water absorption tended to be performing more poorly in the

16 field. It should be noted that Yang research measured the properties of the field concrete

17 long after the concrete was placed so it is difficult to completely separate the initial

18 material properties from the degradation that came over time due to loading and cracking. 
Several recent studies have shown that a relationship exist between the transport

2 properties of concrete and performance. The Virginia Department of Transportation,

3 VDOT (Lane 2006) has recently performed two studies related to the permeability of

4 concrete. The first VDOT study (Ozylindrim 1998) investigated the fabrication and

5 testing of low permeability concrete while the second VDOT study compared different

6 methods of assessing permeability (Lane 2006). VDOT has proposed the development of

7 a database of permeability properties for pavements used in their state (Lane 2006). This

8 would enable permeability properties to be measured and used in quality control

9 procedures and service life prediction. It is anticipated that other states will follow this

10 lead and build their own databases. Once such a database is developed for a state, the

11 state highway engineer can become familiar with the transport properties that they can

12 expect to obtain with their current proportioning and casting processes. Contractors and

13 agencies can then become more familiar with how changes in mixture proportions and

14 new materials lead to either improved or more detrimental behavior. This would enable

15 agencies like INDOT to begin to develop concretes with lower transport properties (e.g.

16 permeability) through mixture proportioning, admixtures, or surface coatings that are

17 specifically aimed at improving long-term performance. This can lead to improved

18 construction practices that lead to improved long-term performance in concrete

19 pavements that would benefit the citizens of the state of Indiana.

20 In addition to providing a database of the materials being used in the state of

21 Indiana, the measurement of permeability (transport) can be used in the development of

22 service life prediction or end result specification models. Recent work in SPR 2941

23 (Barde 2006) has used an approach modeled after the one developed by Fagerlund (1999) 
1 and Bentz et al. (2001) to estimate the service life of concrete pavements in Indiana as a

2 function of air content and secondary sorptivity as shown in Figure 1.1. This model is

3 based on the concept that a pavement which has a lower rate of water absorption would

4 require a longer time to saturate and would therefore be more resistant to freezing and

5 thawing damage resulting in a longer service life. This model also accounts for the role

6 of air content in extending the time required for the concrete to reach a critical saturation

7 level, thereby also extending the service life. The work performed in SPR 2941 was

8 based on literature review and modeling concepts that followed the approach of

$9 \quad$ Fagerlund (1999) and Bentz et al. (2001). As such, paving concrete mixtures and field

10 documented performance were not used to develop these predictions for Indiana. In

11 addition, the work from SPR 2941 used approximations for the degree of saturation that

12 can be expected in concrete using rainfall data (Bentz et al. 2001) rather than measuring

13 saturation directly from the concrete. It is expected that both additional measurement of

14 the permeability properties as well as direct measurements of the degree of saturation in

15 the field can improve these models and their predictions. This work is currently being

16 conducted for INDOT pavement materials as a part of SPR 3200. 


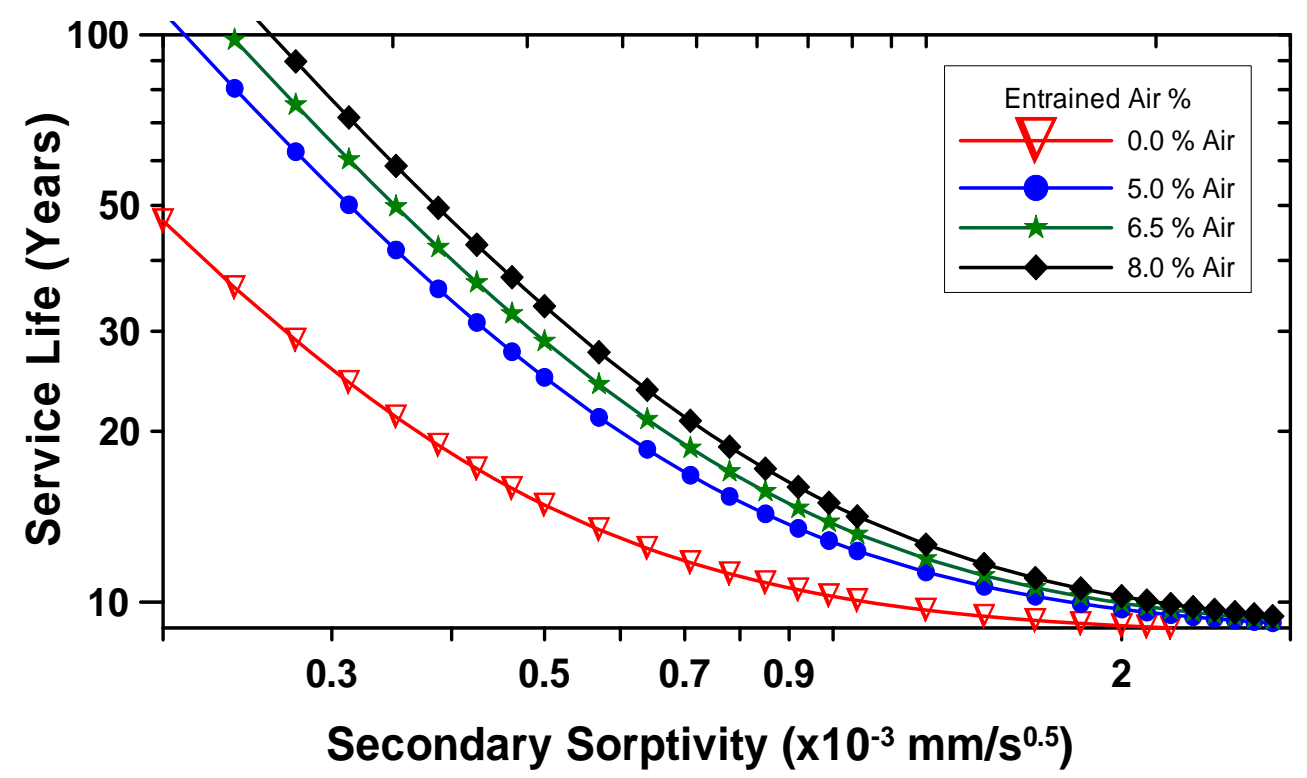

2 Figure 1.1: An Example of the Relationship between the Permeability Properties of a

3 Concrete to its Service Life when Exposed to Freezing and Thawing Cycles (Barde 2006)

While it is generally believed that the transport properties of concrete are related

5 to its long-term durability and performance, there are no common tests or transport

6 specifications used by Indiana for the specification, acceptance, or evaluation of concrete

7 pavements. As such, a need exists to implement a transport test that can be used to

8 develop a database of transport properties of the mixtures used in the state of Indiana.

9 Further, research is needed to determine the current level of permeability (transport) that

10 is achieved and to relate this property with long-term performance.

\section{$\underline{1.2 \text { Problem Statement }}$}

12 This project evaluates the fluid transport properties of concrete pavements

13 currently being placed in the field. Specifically, this work use transport tests for to asses

14 several concrete pavements in Indiana. These tests measured the transport properties of 
1 concrete pavements that are typical for the state of Indiana. These transport properties can

2 eventually be combined with exposure conditions and computer modeling concepts for

3 the purpose of developing performance prediction models. However, at the current time

4 INDOT was performing the study they are only beginning to develop the database of 5 transport properties.

8 greater detail.

9 The second chapter of the report describes a review of literature pertaining to the

10 transport properties of concrete. The main objectives of this chapter are to:

11 1) Assemble a listing of test methods commonly used to measure transport

Chapter 3 reviews experimental results from fourteen different sites/paving 
1 characterize the transport properties of concrete. Originally samples will be tested

2 according to the following procedures:

1) ASTM C-457 to assess the amount of air void in the concrete system,

2) ASTM C-642 to assess the volume of permeable voids,

3) ASTM C-1585 to assess the water absorption of the concrete,

4) Rapid electrical property measurement (Rajabipour 2006),

5) ISO 12572 to assess moisture diffusivity, and

6) Assess moisture absorption and desorption (using the procedure commonly used at Purdue which will be performed on pastes and thin sections due to the time required by this test).

However, after the literature review some of the test methods were updated. ASTM C-457 was updated to an automated method developed after the work of Peterson (2008). ISO 12572 was updated to a more accurate method using a vapor sorption analyzer able to hold relative humidity from $98 \%$ to $0 \%$ at constant temperature. In practical term, this updated method allows to have information for the entire desorption spectra compared with the 2 points spectra provided by ISO 12572 .

Chapter 4 reviews experimental results from mortars prepared in laboratory using different $w / c(0.35,0.40,0.45$ and 0.50$)$. These samples were included in this report because allow a better control of the mixture proportion and the moisture history, which 
1 allows a better assessment of the transport properties. Water absorption based on ASTM

2 C-1585 procedure was used to evaluate these samples.

3 Chapter 5 reports series of wetting and drying tests performed on concrete using

4 different aqueous solutions containing deicing salts. Samples were subjected to different

5 conditioning regimes to evaluate its effect on the test results. This information can be

6 used to better understand water absorption results on samples obtained from the field.

7 Chapter 6 describes a comparison of the ASTM C-1585 testing procedure with a

8 modified version of the ISAT (Initial Surface Absorption Test) for which the data

9 collection can be automated. A comparison of the two methods is presented and

10 statistical significance and repeatability is discussed for each test procedure. This

11 automated test provides additional information compared with the standard ASTM C-

12 1585. This additional data can provide information for further analysis of the absorption

13 behavior of the samples. This test method can also be used to decreases the influence of

14 the operator, and is less time consuming after the test started.

15 Chapter 7 describes the use of electrical properties to assess transport properties.

16 In addition to testing the field samples, a laboratory study was performed to illustrate the

17 influence of minor variations in water and air content on the measured transport

18 properties. Specifically the research illustrates the differences that can be measured in

19 transport properties with 1) production variation, 2) the addition of water as opposed to a

20 water-reducing admixture to maintain slump, and 3) the use of inaccurate daily aggregate

21 moisture contents. This information will be used to developing testing and production

22 variation statements. In addition this will be used for the development of educational

23 materials that will be developed for the concrete paving contractors. 
Chapter 8 reports results from samples exposed to the environment to assess

2 relative humidity and temperature that can be expected in the field. An exposure site was

3 developed in West Lafayette that used embedded sensors. The sample geometry was

4 held constant for the samples on the exposure site using a paving mixture from Indiana

5 with a $w / c$ of 0.42 . Five different exposure conditions were considered to better

6 understand the effects of boundary conditions on the level of saturation that develops

7 inside the pavement. These boundary conditions will include: 1) exposed vertical surface

8 - covered, 2) exposed vertical surface, 3) horizontal surface on a drainable base, 4)

9 horizontal surface on a non draining base, and 5) submerged. In addition, a specimen

10 was exposed to $50 \%$ relative humidity.

11 Chapter 9 discusses the recommendations generated after this study. The

12 information from this report provides the beginning of documentable database for

13 transport properties in the state of Indiana. These results will be combined with results

14 from an ongoing pool funded study to develop testing protocols that can be used in

15 Indiana.

16 


\subsection{Introduction}

3 A literature review on fluid transport was performed. The theory of fluid transport

4 in concrete will be presented first, including a description of the equations used to

5 characterize absorption and permeability. This is followed with a description of the most

6 common tests. The effect of samples preparation is discussed.

8 The ability of concrete to absorb fluid has an influence on its durability.

9 Deterioration processes such as freezing and thawing, sulfate attack, reinforcement

10 corrosion are influenced by the ability of concrete to resist ingress of fluid (Weiss 1999,

11 Nokken and Hooton 2004).

12 Three mechanisms can be used to describe transport of fluid in cementitious

13 systems: (1) permeability, (2) absorption and (3) diffusion. Permeability is the measure of

14 the flow of water under a pressure gradient while diffusion is the movement of ions due

15 to a concentration gradient. Absorption can be described as the materials ability to take in

16 water by means of capillary suction.

17 Transport proprieties of concrete are controlled by the characteristics of its pore

18 network. Total porosity, pore size, pore connectivity, and pore saturation all influence the 
1 measured transport coefficients (Garboczi 1990; Bentz et al. 1999). The pore structure of

2 concrete is complex, spanning six or more orders of magnitude (Bentz et al. 1995). In

3 ordinary portland cement concrete, the largest pores are typically contained in the

4 interfacial transition zones present between cement and aggregate particles (Winslow and 5 Liu 1990).

6 At equilibrium, the saturation of a concrete is determined by the pore structure 7 (size and volume) and the local relative humidity. Many of the pores in concrete are 8 sufficiently small such that condensation will occur at RH values much less than $100 \%$.

9 The relationship between the smallest pore radius which remain water filled $r_{p}$, and $R H$ 10 (for RH greater than $50 \%$ ) is described by the Kelvin-Laplace equation (Equation 2.1) 11 (Bentz et al 1995):

$12 \ln \left(\frac{R H}{100}\right)=-\frac{2 V_{m} \sigma}{r_{p} R T}$

13 where $V_{m}$ is the molar volume of water, $\sigma$ is the surface tension of the liquid, $R$ is the 14 universal gas constant, $\mathrm{T}$ is the absolute temperature in degree Kelvin.

15 The validity of this equation for RH lower than $50 \%$ is questionable due the fact 16 that the water meniscus would be composed of only about ten molecules. A considerable 17 hysteresis exists in the adsorption/desorption curves, due to ink-bottle and pore topology 18 effects. Thus, simply knowing the RH of a concrete is insufficient for making an accurate 19 estimate of its moisture content (Martys 1995, Rajabipour and Weiss 2006). 
3 into contact with a wetting liquid phase, the liquid will invade due to the capillary forces

4 present in each pore. The local capillary force is inversely proportional to the pore

5 diameter, with smaller pores exerting a large capillary force (although the rate of ingress

6 into a smaller pore will actually be less than that into a large one).

$7 \quad$ For the case of one-dimensional flow, absorption of concrete is generally defined

8 by its sorptivity, S, determined using Equation 2.2 (Martys 1995):

$9(t)=\rho A S \sqrt{t}$

$$
M(t)=\rho A S \sqrt{t}
$$

10 where $M(t)$ represent the mass of liquid gained by the specimen at time $t, \rho$ is the density

11 of the invading liquid, $\mathrm{A}$ is the surface area of the specimen exposed to the liquid, and $\mathrm{S}$

12 is the sorptivity in units of length/time ${ }^{0.5}$. For the field concrete, the square root of time

13 dependency may not always be followed, as the exponent in the power law function may

14 vary between 0.2 and 0.5 (Sosoro 1998).

15 In practice, it is often observed that there is a rapid initial absorption so the

16 Equation 2.2 is modified to Equation 2.3 (Parrot 1994):

17

$$
M(t)=M_{o}+\rho A S \sqrt{t}
$$


1 where Mo represents the initial mass gain.

2 Deviation from the square root of time behavior at longer times has been observed

3 in numerous experiments (Sosoro 1998). It is generally attributed to interactions of water

4 with the concrete. Hall and Yau (1987), and Martys and Ferraris (1997) have proposed

5 modifications to Equation 2.3 to account for these long time deviations. The proposed

6 equation of Hall and Yau (Equation 2.4), has the form:

7

$$
M(t)=M_{o}+\rho A\left(S \sqrt{t}-C_{H} t\right)
$$

8 The proposed equation of Martys and Ferraris (Equation 2.5) has the form:

9

$$
M(t)=M_{o}+\rho A\left[S_{g} \sqrt{t}+C_{M}\left(1-\exp \left(\frac{-S \sqrt{t}}{C_{M}}\right)\right)\right]
$$

10 where $\mathrm{C}_{\mathrm{H}}$ and $\mathrm{C}_{\mathrm{M}}$ are constants obtained from fitting the experimental data, and $\mathrm{S}_{\mathrm{g}}$

11 describes the sorptivity in the smaller pores or the effects of moisture diffusion.

13 Permeability of concrete plays an important role in durability because it controls

14 the movement and the rate of entry of water, which may contain aggressive chemical 
1 (Bamforth 1987). Permeability is characterized by the permeability coefficient k. It can 2 be measured as gas permeability and liquid permeability.

\section{$3 \quad$ 2.2.2.1 Gas Permeability}

4 To measure gas permeability in the most direct manner, a pressure is applied 5 across a concrete specimen and the flow rate at steady is measured. With this setup, the 6 gas permeability, $\mathrm{kg}_{\mathrm{g}}$, is given Equation 2.6 (Bamforth 1987):

$7 \quad k_{g}=\frac{2 Q x \eta P_{f}}{A\left(P_{1}^{2}-P_{2}^{2}\right)}$

8 where $\mathrm{P}_{1}$ and $\mathrm{P}_{2}$ are the upstream and downstream pressures respectively, $\mathrm{Q}$ is the

9 volumetric flow rate in $\mathrm{m}^{3} / \mathrm{s}, \mathrm{x}$ is the specimen thickness in $\mathrm{m}, \mathrm{A}$ is the area for flow in

$10 \mathrm{~m}^{2}$, and $\mathrm{kg}$ is the permeability coefficient in units of $\mathrm{m}^{2} . \mathrm{P}_{\mathrm{f}}$ correspond to the pressure at

11 which the flow rate is measured, either $\mathrm{P}_{1}$ or $\mathrm{P}_{2}$, depending on the experimental setup.

12 Gas permeability can be also calculated using the modified Darcy's equation

13 (Equation 2.7), as proposed by Grube and Lawrence (Grube and Lawrence 1984):

$14 \quad \mathrm{~K}_{\mathrm{o}}=\frac{2 \mu \mathrm{p}_{\text {out }} \mathrm{QL}}{\mathrm{A}\left(\mathrm{p}_{\text {in }}^{2}-\mathrm{p}_{\text {out }}^{2}\right)}$ 
1 where $\mathrm{Q}$ is volume flow rate $\left(\mathrm{m}^{3} / \mathrm{s}\right), \mathrm{L}$ is sample thickness $(\mathrm{m}), \mathrm{p}_{\text {in }}$ is pressure at inlet

2 (bar), $p_{\text {out }}$ is pressure at outlet (bar), and A is cross-sectional area of sample $\left(\mathrm{m}^{2}\right)$.

\section{$3 \quad$ 2.2.2.2 Liquid Permeability}

$4 \quad$ There exist a wider set of proposed equation for measurement of liquid

5 permeability. According with Bamforth (1987), the governing equation for determining

6 intrinsic permeability is (Equation 2.8):

$7 \quad k_{l}=\frac{Q \times \eta}{A\left(P_{1}-P_{2}\right)}$

8 where all terms were previously defined.

9 According with Shafiq and Cabrera (2004), the intrinsic coefficient of water

10 permeability can be calculates using the Equation 2.9:

$11 \quad \mathrm{~K}_{\mathrm{w}}=\frac{\mathrm{d}^{2} v}{2 \mathrm{th}}\left(\frac{\mu}{\rho g}\right)$

12 where $d$ is the depth of water penetration $(\mathrm{m}), \mathrm{t}$ is the time of penetration $(\mathrm{s}), \mathrm{h}$ is the 13 applied pressure (m), $v$ is the total porosity (fraction), $\rho$ is the density of water $\left(\mathrm{kg} / \mathrm{m}^{3}\right), \mu$

14 is the viscosity of water $\left(\mathrm{Ns} / \mathrm{m}^{2}\right)$, and $\mathrm{g}$ is the acceleration due to gravity $\left(\mathrm{m} / \mathrm{s}^{2}\right)$.

15 Katz and Thompson (1986) proposed the relationship show in Equation 2.10: 
$1 \quad \mathrm{~K}=\mathrm{c} \cdot \mathrm{d}_{\mathrm{c}}^{2} \cdot\left(\frac{\sigma}{\sigma_{o}}\right)$

2 where $\mathrm{K}$ is the permeability $\left(\mathrm{m}^{2}\right), \mathrm{d}_{\mathrm{c}}$ is the critical pore diameter $(\mathrm{m}), \sigma$ is the electrical 3 conductivity of the sample $(\Omega-\mathrm{cm})^{-1}, \sigma_{\mathrm{o}}$ is the conductivity of the pore fluid $(\Omega-\mathrm{cm})^{-1}$, and $4 \mathrm{c}$ is a constant.

5 To predict the permeability of a material using the Katz-Thompson equation, the

6 critical pore diameter $\left(d_{c}\right)$ and the normalized conductivity $\left(\sigma / \sigma_{0}\right)$ must be measured. Katz

7 and Thompson related the critical pore diameter to the inflection point in mercury

8 intrusion curves. The physical interpretation of the critical diameter is that it is the

9 smallest continuous pore size that percolates through the sample. The normalized

10 conductivity is determined using electrical methods.

11 According to Shane et al. (1997), the main advantage of the Katz-Thompson

12 relation is that the time required to make these measurements is much shorter and much

13 less labor intensive than is required to make permeability measurements. Also, unlike

14 with permeameters, there is not a limit of permeability that can be predicted, once set has

15 occurred, and $d_{c}$ can be measured. This allows specimens of any age, or composition, to

16 be evaluated.

\section{$17 \quad$ 2.2.2.3 Gas Permeability vs. Liquid Permeability}

18 When comparing gas permeability to liquid permeability, it is necessary to correct

19 for the slippage of the gas, commonly referred as the Klinkenberg correction (Nokken 
1 and Hooton 2004). By measuring the gas permeability under a variety of pressure

2 gradients, the intrinsic permeability (permeability at infinite pressure) can be derived by

3 fitting the data to an equation of the form (Equation 2.11):

$4 \quad k_{l}=\frac{k_{g}}{1+\frac{b}{P_{m}}}$

5 where $k_{1}$ is the intrinsic permeability, $P_{m}$ is the mean pressure of the inlet and outlet

6 streams, and $\mathrm{b}$ is a constant for a given gas and a given concrete.

\subsubsection{Diffusion}

8 The present section provides a method to calculate diffusivity, the coefficient of

9 moisture diffusion, from weight loss of a sample exposed to controlled conditions (i.e.,

10 relative humidity and temperature).

\section{$11 \quad$ 2.2.3.1 Moisture Desorption Technique}

12 Moisture desorption is an established technique for evaluating the effect of

13 moisture loss at a given humidity for a material. A TA Q5000 SA moisture sorption

14 analyzer was used in this investigation (Figure 2.1). The system enables the desorption

15 behavior to be evaluated under carefully controlled conditions of temperature and

16 humidity (Castro et al 2010a). 


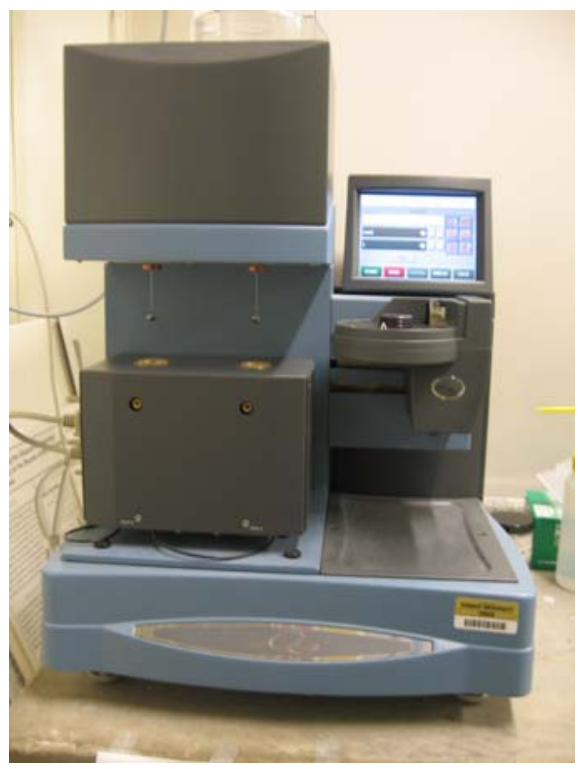

Figure 2.2 shows an example of the results that can be obtained from moisture

4 desorption. As the relative humidity is changed, the sample undergoes a rapid change in

5 mass. The mass change decreases as the sample approaches equilibrium. It can be seen

6 that this general behavior is observed at each change in the relative humidity, however,

7 the magnitude of the mass change is different at each relative humidity and would be

8 consistent with the volume of pores from which water is being lost at each step. 
7 since the change in $\mathrm{RH}$ value is small. The change in mass of sample, $\mathrm{M}_{\mathrm{t}}$, as a function of time, t, can be represented using Equation 2.12 (Crank 1980):

$9 \quad \frac{M_{4}}{M_{x}}=\frac{4}{\pi}\left(\frac{\partial E}{L^{2}}\right)^{2 / 2}$

10 where $M_{\infty}$ is the mass change at equilibrium, $D$ is is the diffusivity and $L$ is the thickness

11 of the sample. Equation (2.12) can be used to obtain the $D$ at each step by calculating the

12 slop of a curve that is obtained by plotting $\frac{M_{C}}{M_{\infty}}$ verses square root of time. 


\section{$2 \quad$ 2.2.4.1 Water Content}

3 The water to cement ratio is generally considered to be the governing parameter,

4 which affects the strength and durability of concrete. When a high water to cement ratio

5 is used in concrete it will generate a higher initial porosity. However the threshold values

6 are very dependent of the curing regime will lead premature deterioration of concrete

$7 \quad$ structures has created (Figg 1973).

\section{$8 \quad$ 2.2.4.2 Supplementary Material}

$9 \quad$ a) Silica Fume

10 According to Bentz (2000), silica fume influences concrete diffusivity in several

11 ways: (1) by densifying the microstructure if the interfacial transition zone, (2) by

12 reducing the overall capillary porosity for a fixed degree of cement hydration, and (3) by

13 production a pozzolanic $\mathrm{C}-\mathrm{S}-\mathrm{H}$ gel with a relative diffusivity about 25 times less than that

14 of the C-S-H gel produced from conventional cement hydration. This beneficial effect is

15 observed regardless of the temperature of the curing regime (Cabrera 1996).

16 b) Slag

17 Because of their low heat of hydration, slag cements help to prevent cracks in 18 mass concrete structures resulting from temperatures stresses at early ages (Geiseler et al. 
1 1995). For this reason, slag replacement offers stronger and more durable concrete in hot

2 climates. According to Bleszynski et al. (2002), the incorporation of blast-furnace slag

3 into silica fume concrete reduces the water demand. It can lead also to a lower initial

4 porosity of the concrete with its advantages to reduce durability problems.

$5 \quad$ c) Fly Ash

6 Fly ash, a by-product of the combustion of pulverized coal in thermal power

7 plants, is commonly used in concrete as a partial replacement for Portland cement. Fly

8 ash is capable of reacting with the calcium hydroxide $(\mathrm{CH})$ produced during cement

9 hydration to form calcium silicate hydrate (CSH) which fills large capillary voids and

10 disrupts their continuity. Class $\mathrm{F}$ fly ash reduces permeability even at relatively low

11 levels of cement substitution (10\% by weight). In the case of Class C fly ash however,

12 relatively high cement substitution level (20 to $30 \%$ by weight) may be required to

13 produce any significant reduction in permeability (Alhozaimy et al. 1996).

\section{$14 \quad$ 2.2.4.3 Effect of Curing}

15 Several researchers have studied the relationship between permeability and pore

16 size distribution of cement paste or mortar. They concluded that the flow of a fluid

17 through concrete is associated with the larger capillary pores rather than total porosity.

18 Pore volume above a certain size pore (in the region of $880-1500 \mathrm{~A}$ ) is thought to be

19 closely related to permeability (Gowripalan et al. 1990). 
1 The rate of chloride ingress through the concrete cover will be influenced by the

2 type and extend of curing. A lower degree of hydration of the cementing materials close

3 to the surface will result in higher porosity and a more connected capillary pore system

4 and, therefore, diffusion coefficients through the cover depth will be spatially dependent.

5 As periods of moist curing are extended, the depth-dependent effect on diffusion

6 coefficient is reduced (Hooton et al. 2002).

7 According to Wang et al. (2006) regardless of the application of curing

8 compound, properties of the near-surface layer concrete, such as moisture content,

9 permeability, degree of hydration, and sorptivity, differ from those of the internal

10 concrete. However, application of a curing compound significantly increase degree of

11 cement hydration and decreases sorptivity of the near-surface layer concrete, which

12 reduces the difference in concrete properties between the near-surface layer and internal

13 concrete.

14 The effectiveness of initial curing becomes more important when mineral

15 admixtures like fly ash are used as partial substitution for cement concrete (Shafiq and

16 Cabrera 2004).

$18 \quad$ 2.2.5.1 Degree of Saturation and Relative Humidity

19 Both sorptivity and gas permeability are strongly influenced by the water content

20 of the concrete being evaluated (Abbas et al. 1999). As the water content is increased, 
1 more and more of the pores are filled with liquid water, which significantly alters both

2 the sorption liquid water and the transport gas. Thus, even in laboratory testing, the pre-

3 conditioning of the specimens is of paramount importance (Boddy et al. 1999). For liquid

4 permeability, the samples are normally vacuum saturated. Details of sample preparation

5 will not be discussed in this section.

$6 \quad$ Many different samples preparation methods had been used for sorptivity and gas

7 permeability. During the late 80 's and early 90 's drying at $105{ }^{\circ} \mathrm{C}$ was used as the

8 conditioning method (Dhir et al. 1989). However, it is now well recognized that this

9 method produces a moisture content that is atypical with respect to field conditions

10 (Boddy et al 1999). Additionally, the elevated temperature will lead to severe drying of

11 the cement gel that will alter the microstructure of the cementitious binder resulting in

12 elevated values for sorptivity (Parrot 1994).

13 The Cembureau method (Kollek 1986) recommended that one of the following

14 two pre-conditioning regimes be employed: 1) storage in a laboratory atmosphere at 20

$15{ }^{\circ} \mathrm{C}$ and $65 \% \mathrm{RH}$ for 28 days or 2) drying in a ventilated oven at $105{ }^{\circ} \mathrm{C}$ for 7 days

16 followed by storage in a desiccator for 3 days at $20{ }^{\circ} \mathrm{C}$. However, it is well recognized

17 that it may take several months to obtain moisture equilibrium in specimen only a few

18 centimeters thick

In 1994 Parrot (1994) conducted a series of experiments to analyze conditioning

20 procedures and assess the moisture distribution of concrete samples. The Parrot (1994)

21 work used $100 \mathrm{~mm}$ cubes of concrete with a cast-in cylindrical cavity. These samples

22 were used to measure the differences in relative humidity between the inside and the

23 outside of the sample. That research reported that drying for 4 days at $50{ }^{\circ} \mathrm{C}$ followed by 
13 days of sealed storage at $50{ }^{\circ} \mathrm{C}$ caused a small relative humidity gradient in the concrete

2 cube that gradually diminished when the specimens were placed in a sealed container at

$320^{\circ} \mathrm{C}$ for 25 days.

4 The sample conditioning established by ASTM C-1585 (2004) consist of placing

5 the specimens in an environmental chamber at a temperature of $50 \pm 2{ }^{\circ} \mathrm{C}$ and $\mathrm{RH}$ of $80 \pm$

$63 \%$ for 3 days. Alternatively, the specimens can be placed in a desiccator inside an oven

7 at temperature of $50 \pm 2{ }^{\circ} \mathrm{C}$ for 3 days. If the desiccator is used, the $\mathrm{RH}$ is controlled

8 using a saturated solution of potassium bromide. After 3 days, the specimens are placed

9 inside a sealable container for at least 15 days at $23 \pm 2{ }^{\circ} \mathrm{C}$. The total drying period of 18

10 days recommended in the standard is reasonable and certainly more efficient than the

11 several month required for the equilibrium of moisture when conditioning chambers are

12 used.

13 When the standard recommendation is followed, specimens should obtain a 14 humidity between $50 \%$ and $70 \%$. However, Nokken and Hooton (2002) established

15 experimentally that the rate of absorption is highly dependent of the actual degree of

16 saturation. Then, if a sorption test is performed on twin specimens conditioned at RH of

$1750 \%$ and $70 \%$, the rate of absorption will be different.

\section{$18 \quad$ 2.2.5.2 Temperature}

19 Temperature influences sorptivity through its effect on the surface tension $(\sigma)$ and

20 the dynamic viscosity of the fluid $(\eta)$. Hall indicates that absorption should scale as

$21(\sigma / \eta)^{0.5}$ (Hall 1994). Using this relationship, measurements made at different temperatures

22 can be normalized to a common standard temperature for comparative purposes. 
While this scaling relationship holds well for the absorption of organic fluids by

2 concrete, significant deviations from the theory are observed when water is used as the

3 sorbing fluid (Sosoro 1998). This could be due to the changing solubility of calcium

4 hydroxide with temperature and the complex interactions of water with the concrete 5 matrix (Boddy et al. 1999).

\section{$6 \quad$ 2.2.5.3 Critical Analysis of Effect of Samples Preparation}

7 It appears clear that sample preparation could be an important issue when sorption 8 test are used to characterize different mixtures. Following the standard procedure, the 9 sample preparation is reported to result in a relative humidity between $50 \%$ and $70 \%$, 10 however this is a wide range.

11 The main factor that will affect the final moisture content in samples after 12 standard preparation will be the initial moisture content. However, not work was found 13 about this topic. It will be a very interesting area for future research.

14 With respect to temperature not too much research had been performed on the 15 absorption at low temperatures. A consensus appears to exist to report sorptivity results at $1623{ }^{\circ} \mathrm{C}$. However it could be important develop a better understanding of what happens in 17 terms of absorption and desorption of fluids when temperatures are close to freezing 18 point. 
3 harmful materials into the concrete. Consequently, durability can be improved 4 significantly by producing concrete that is highly resistant to the ingress of external

5 harmful substances as Mehta said: "impermeability of concrete should be the first line of

6 defense against any of the physiochemical deterioration processes" (Mehta 1994).

7 Therefore, the evaluation of the permeability in a concrete structure is an essential

8 and important step for the definition of its durability, performance and service life. This

9 report is intended to be brief description of some of the most commonly used methods

10 and their advantages and disadvantages. One general point that must be borne in mind

11 when discussing these tests is that few of them measure absolute values of fundamental

12 transport properties; what they do provide is an index or relative value and this is useful

13 for comparing one concrete with another.

15 Measuring the gas permeability of concrete is relatively quick and enables

16 specimens conditioned at any age to be tested without any time delay. In addition, the

17 conditions of the specimen do not change during the testing. For these reasons these tests

18 have become quite common in order to measure the permeability of concrete. Testing

19 concrete for gas permeability can be carried out by either keeping the head constant and

20 measuring the flow or by monitoring the pressure decay over a specified time interval

21 (Basheer 2001). 


\subsubsection{Sch nlin Method}

The method consists of placing a cell, equipped with a rubber gasket, on the

3 concrete surface and creating vacuum. Figure 2.3 shows a schematic illustration of this

4 method. Once a certain vacuum has been reached, the stopcock is closed and the time

5 elapsed for the absolute pressure to rise from 50 to $300 \mathrm{mbar}$ is recorded. These

6 measurements allow the calculation of a permeability index, which is defined as the

7 airflow into the chamber divided by the average chamber depression. The method is very

8 fast ( $\sim 3$ minutes) and easy to perform and it is entirely non-destructive.

9 This test is considerably influenced by the moisture condition of the concrete. To

10 eliminate this problem, Schönlin and Hilsdorf (1987) suggested that the concrete surface

11 should be dried with hot air for 5 min before the start of a test.

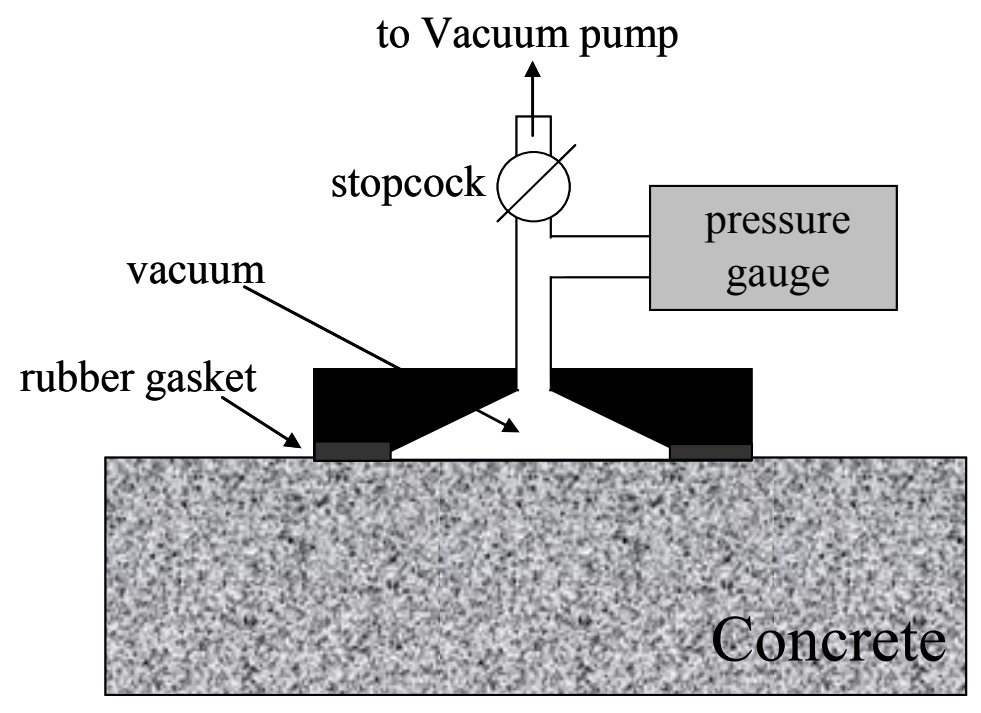

12

Figure 2.3: Schematic View of the Sch nlin Method 


\section{$1 \quad$ 2.3.1.2 Surface Air Flow Test}

3 industry to determine the permeability of rock cores (Whiting and Cady 1992). A

4 vacuum plate is placed on the concrete surface. Valves A, B, and C (Figure 2.4) are

5 closed. The vacuum pump is started and allowed to stabilize at a vacuum pressure of

6 approximately $-83 \mathrm{kPa}$. Valve $\mathrm{A}$ is then opened allowing the vacuum to be created inside

7 the vacuum plate. After the vacuum has been created, valve A is closed and valve B is

8 opened. To maintain the vacuum pressure, the air that has permeated into the vacuum

9 chamber through the concrete is drawn out through the flow meter. After a period of

10 approximately 15 seconds, to allow the system to stabilize, the flow rate of the air is

11 measured by a flowmeter. This flow rate is used as a measure of the surface air

12 permeability. Similar to the Schönlin test, if the surface layer has high moisture content,

13 the surface should be dried with hot air. 
10 portable and easy to use on site. Non-skilled personnel can carry out the tests. The tests

11 can be carried out without leaving any mark on the test surface (Figure 2.5).

12 The total duration of each test is less than 30 minutes, which includes instrument 13 setting up time.

Up to 12 tests can be carried out using the battery pack available within the 15 instrument or run continually during the day if an external power supply is available. The 
1 data from each test is stored in the controller of the instrument and can either be recorded

2 manually or transferred to a PC for further analysis (Amphora NDT Limited 2009).

3 After the body of the apparatus is placed on the based ring, the pressure in the test

4 area is increased to $1.5 \mathrm{bar}$, using the syringe attached the priming valve. The pressure

5 decay is monitored for 15 minutes (or until the pressure reaches zero) with 1 minute

6 interval. The slope of the pressure-time curve between 5 th and 15 th minute is used as the

7 air permeability index. The test should be not carried out if the internal relative humidity

8 of the covercrete is greater that $80 \%$. This should be considered in light of the results

9 presented in Chapter 8.

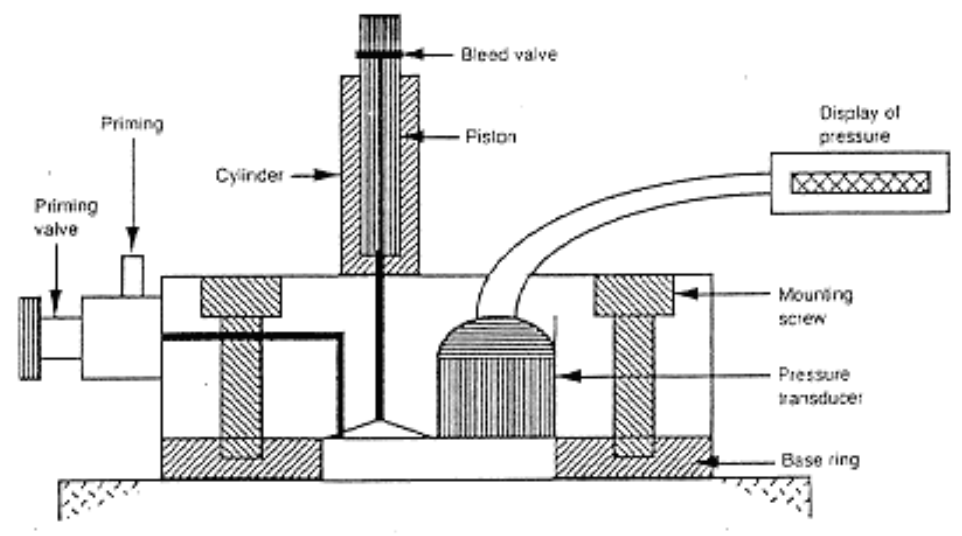

10

11 Figure 2.5: Autoclam Apparatus for Permeability Tests (Zia et al. 1994)

$12 \quad$ 2.3.1.4 Torrent Permeability Tester (Torrent 1992; Mastrad Limited 2009; Proceq 2009)

13 The TORRENT permeability tester is based on investigations which were carried

14 out by the research center of "Holderbank Management and Consulting Ltd.",

15 Switzerland (Figure 2.6). 
The particular features of the Torrent method are a two-chamber vacuum cell and

2 a pressure regulator. This ensures that an air flow at right angles to the surface is directed

3 towards the inner chamber. The cell is placed on the concrete surface and a vacuum is

4 created in both chambers with pump. Due to the external atmospheric pressure and the

5 rubber rings, the cell is pressed against the surface and thus both chambers are sealed.

6 After 1 minute, the inner chamber is insulated. From this moment, the pressure in the

7 inner chamber starts to increase, as air is drawn from the underlying concrete. The rate of

8 pressure raise, which is directly related to the permeability of concrete, is recorded.

9 Meanwhile, the vacuum pump continues to operate on the outer chamber to keep the

10 pressure equal in both chambers. The permeability coefficient can then be calculated

11 (Table 2.1). 


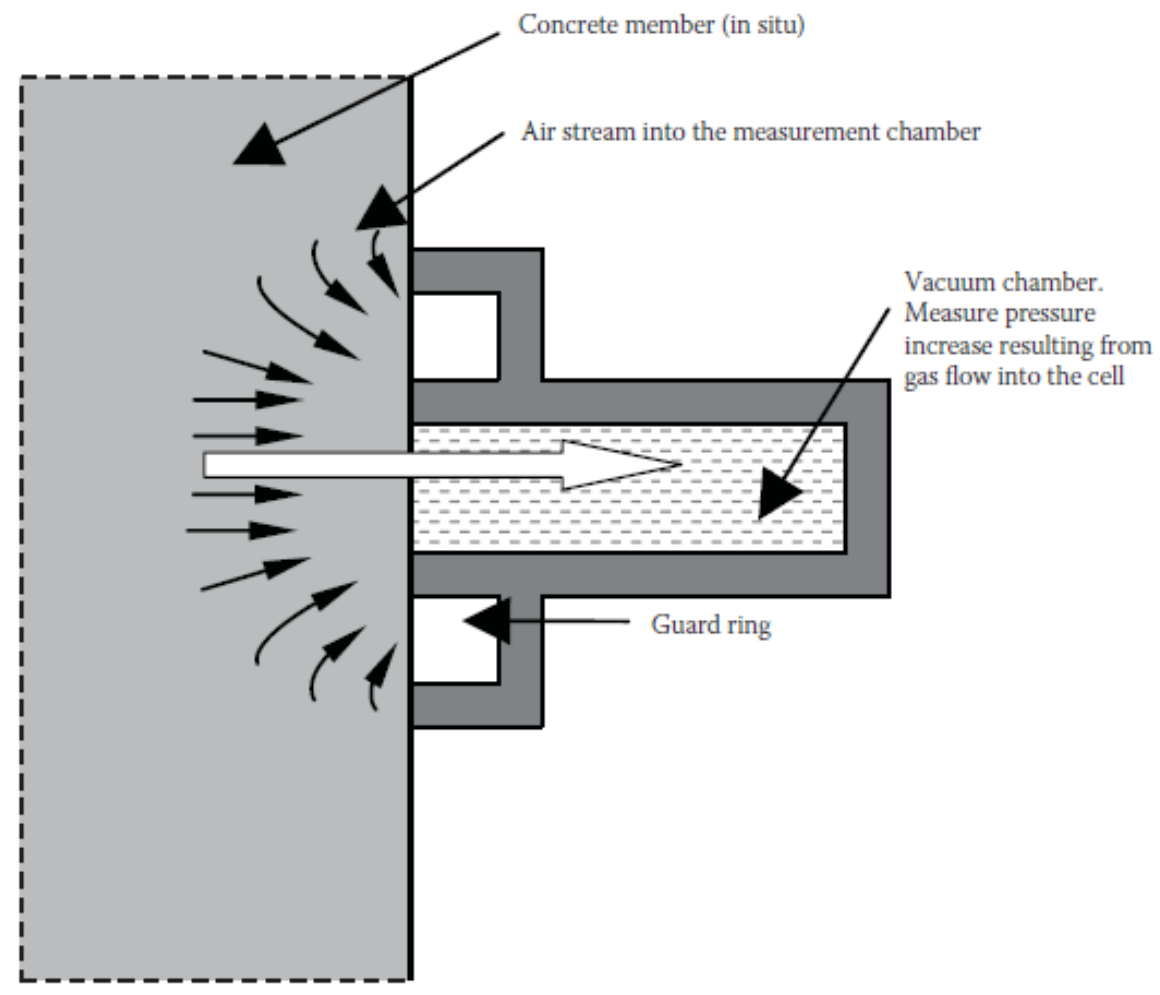

Figure 2.6: Schematic of Torrent Test Setup

Table 2.1: Concrete Categories Based on Torrent Permeability Coefficient

\begin{tabular}{|c|c|c|}
\hline Concrete category & Covercrete quality & $\begin{array}{c}\text { Torrent permeability coefficient }(\mathrm{kT}) \\
\text { after } 28 \text { days }\left(\times 10^{-16} \mathrm{~m}^{2}\right)\end{array}$ \\
\hline 1 & excellent & $\mathrm{kT}<0.01$ \\
\hline 2 & good & $0.01<\mathrm{kT}<0.1$ \\
\hline 3 & fair & $0.1<\mathrm{kT}<1$ \\
\hline 4 & not very good & $1<\mathrm{kT}<10$ \\
\hline 5 & poor & $\mathrm{kT}>10$ \\
\hline
\end{tabular}


The measurement takes 2-12 minutes, depending on the permeability of the

2 concrete. In the case of dry concrete, the quality class of the concrete cover can be read

3 from a table using the $\mathrm{kT}$ value. In the case of moist concrete, $\mathrm{kT}$ is combined with the

4 electrical concrete resistance $\mathrm{R}(\mathrm{Ohm})$ and the quality class is determined from a

5 nomogram. The test is completely non-destructive, very easy to handle

$6 \quad$ 2.3.1.5 TUD Method (Reinhardt-Mijnsbergen) (Torrent et al. 2007)

7 The method is consisted of drilling a small hole with diameter of $10 \mathrm{~mm}$ and the

8 depth of $40 \mathrm{~mm}$ on the surface of the concrete. A cylindrical hollow probe is introduced

9 to the hole. After sealing the hole the chamber the nitrogen gas at the pressure of 10 to

1010.5 bar is introduced through the probe. After, shutting the gas flow, the time required

11 for a pressure decay in the chamber from 10 to 9.5 bar is recorded. Time for test

12 including setting up time is about 20 minutes. This method is slightly destructive (Figure

13 2.7). 


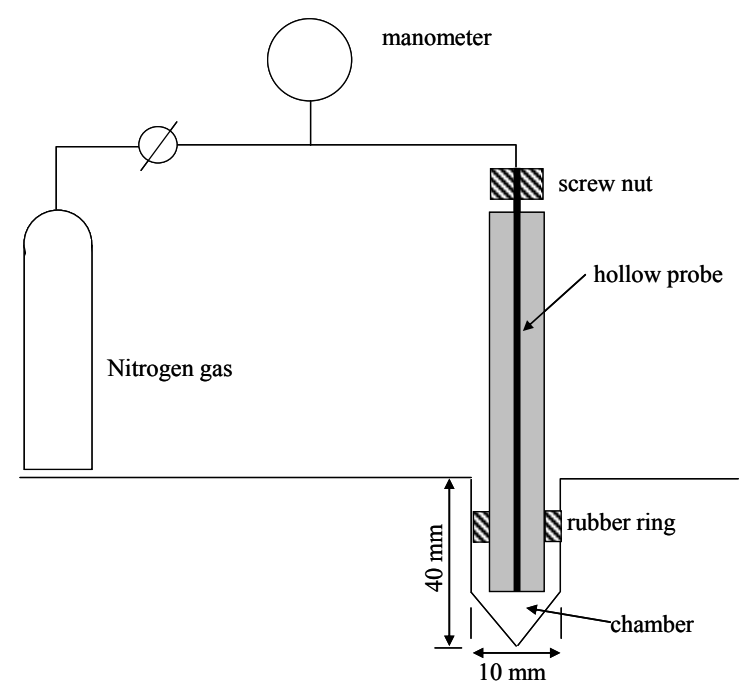

Figure 2.7: Schematic of the TUD Method

$3 \quad$ 2.3.1.6 Hong-Parrott Method (Parrot and Hong 1991, Torrent et al. 2007)

$4 \quad$ It consists in drilling a blind hole, with the depth of $35 \mathrm{~mm}$ and diameter of 20

$5 \mathrm{~mm}$, into the concrete surface. The hole is sealed with stainless steel and silicon rubber

6 plug. A pressure transducer and a digital indicator are connected to the plug. The hole is

7 pressurized with air slightly above 1 atmosphere, and the time taken for the relative

8 pressure to drop from 50 to $35 \mathrm{kPa}$ is measured. Soap solution is brushed on the concrete

9 surface around the hole which produces fine bubbles as airflows across the surrounding

10 concrete. If the concrete is cracked or poorly compacted, large bubbles can be seen. The

11 measured time is converted into an apparent permeability, as function of the radius

12 affected by the test (revealed by bubbles in the soap solution). Time for test including

13 setting up time is about 40 minutes. This method is slightly destructive (Figure 2.8). 


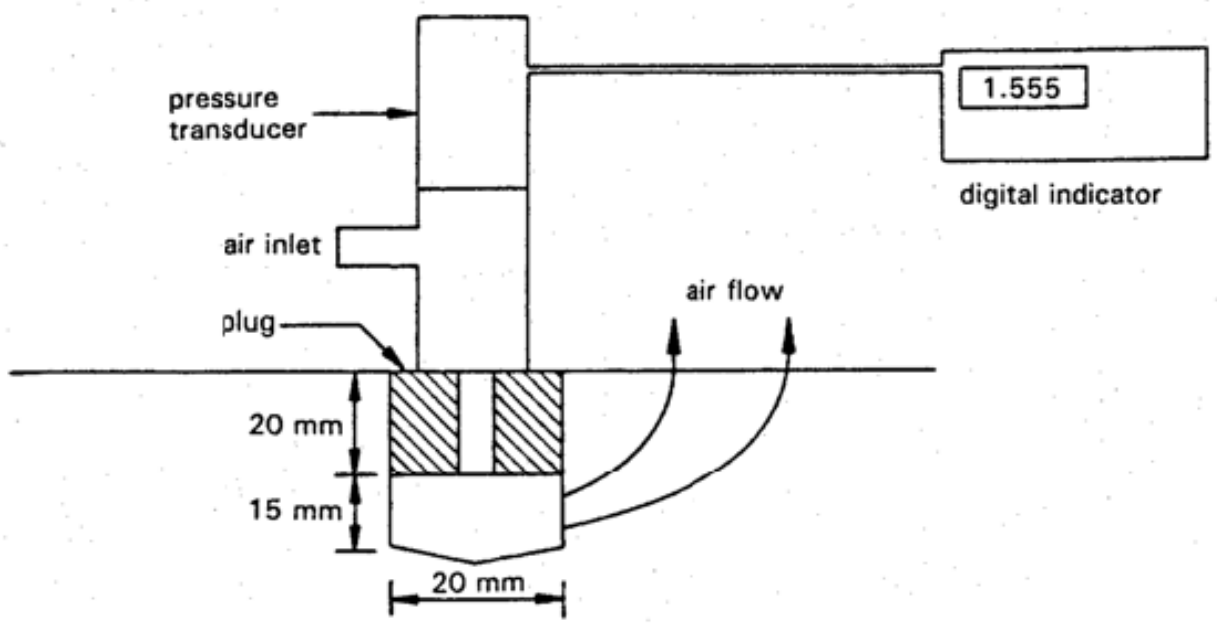

Figure 2.8: Schematic View of Hong-Parrott Method

$3 \quad$ 2.3.1.7 Germann's Gas Permeation Test (Hanson et al. 1984; Germann Inst. Inc. 2009)

4 A hole is drilled into the surface at an angle of 45 degrees and a pressure sensor is

5 installed at in the hole. $\mathrm{CO}_{2}$ gas is pressurized to the surface and the sensor pressure

6 recorded over time. The permeation relative to the porosity of the concrete is determined

7 by recorded graphs. The test rig illustrated is secured to the surface by means of two

8 adjustable, anchored clamping pliers. The rig contains a gasket being pressurized against

9 the surface. If needed, the gasket may be glued to the face. Coring of the sensor hole

10 takes place with a water-cooled diamond drill bit at a given distance from the gasket (15,

1120 or $25 \mathrm{~mm})$. The sensor is inserted in the cored hole, expanded and checked for air-

12 tightness. The $\mathrm{CO}_{2}$ surface pressure is 1,2 or 3 bar and the time of testing is normally 2,

135 or 10 minutes. Alternatively, oxygen can be used (Figure 2.9). 
6 specimen (typically $150 \mathrm{~mm}$ diameter by $50 \mathrm{~mm}$ thick) is caused by a constant absolute

7 pressure difference of the test gas between the two surfaces. The testing should be

8 carried out in a laboratory with $\mathrm{T}=20 \pm 2{ }^{\circ} \mathrm{C}$ and $\mathrm{RH}=65 \pm 5 \%$. It is recommended to

9 determine the pressure of five absolute inlet pressure stages as: 1.5, 2, 2.5, 3 and 3.5 bar.

10 At each stage, the flow rate should be allowed to stabilize, which normally achieved

11 within 5-30 minutes. The mean specific coefficient of permeability is evaluated from the

12 five permeability coefficient values obtained for the five pressure stages. The gas flow

13 depends on the pressure difference, testing area, specimen thickness and open porosity,

14 and the viscosity of the test gas (Figure 2.10). 


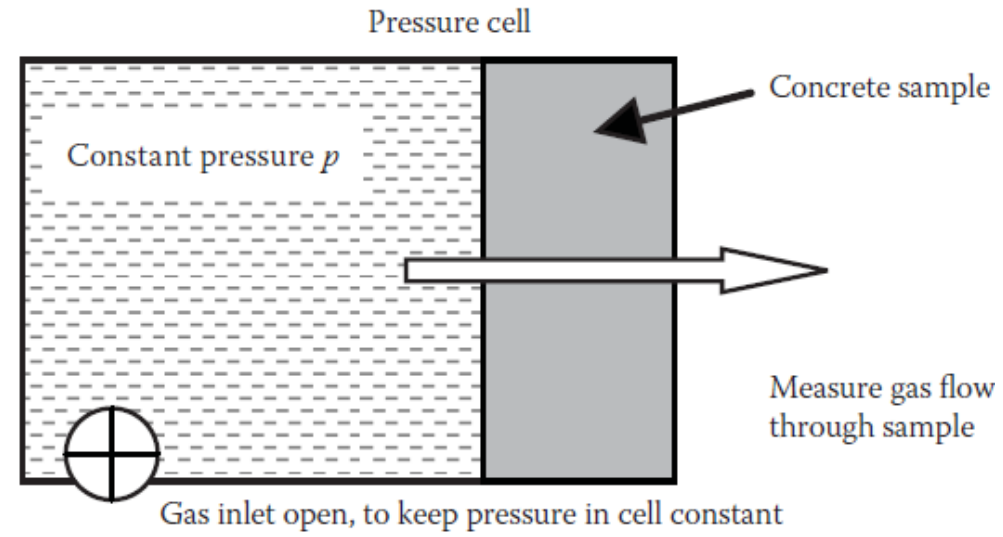

Figure 2.10: Schematic of Cembureau Method

5 absorption tests and permeation tests.

7 Under perfect conditions, the magnitude of capillary rise follows a linear relationship 8 with the square root of time elapsed, and the constant of proportionality is called the

9 sorptivity and ideally, this is the property that should be measured in an absorption test

10 (Basheer 1993). However, there are always practical limitations such as: difficulty in 11 achieving a unidirectional penetration of water, problems of determining the water

12 penetration depth without actually splitting the concrete specimen, and etc. Therefore, 13 the absorption characteristics of concrete are usually measured indirectly by one of the 14 following types of test.

15 Permeability is the property of concrete that describes resistance to a fluid 16 penetration under the action of a pressure gradient (The Concrete Society 1988; Perraton 
1 and Aitcin 1992). Sometimes, permeability is confused with porosity, and vice-versa.

2 Porosity is simply a measure of the proportion of the total volume occupied by pores,

3 usually expressed as a percentage (Neville 1995).

\section{$4 \quad$ 2.3.2.1 ISAT (Initial Surface Absorption Test) (Basheer 2001)}

5 The ISAT was originally developed by Glanville in 1931 and modified by Levitt

6 in 1970s (Levitte 1971). The test starts with placing an acrylic cap onto the concrete

7 surface with a minimum water contact area of $5000 \mathrm{~mm}^{2}$ and making it water-tight by

8 clamping it (Figure 2.11). A pressure head of $200 \mathrm{~mm}(\sim 0.02$ bar) is set by mean of

9 water reservoir. With the inlet tap opened, water flows to fill the gap and then through

10 the outlet it climbs into the calibrated horizontal capillary tube. After $10 \mathrm{~min}$, the tap is

11 closed and the rate of water suction by the concrete is monitored by following the

12 retraction of the meniscus in the capillary tube. The absorption values are determined in

13 this manner at 10, 30, 60 and 120 minutes from the start of the test. The inlet tap is

14 opened after each measurement in order to allow the head of the water to be maintained

15 at $200 \mathrm{~mm}$. ISA-value is given by Equation 2.13 .

$16 \quad \mathrm{ISA}=0.6 \mathrm{D} / \delta \mathrm{t}$

17 where ISA $=$ rate of water suction $\left(\mathrm{ml} / \mathrm{m}^{2} / \mathrm{s}\right)$ and $\mathrm{D}=$ Number of scale the meniscus

18 moves during time $\delta$ t. The test results are evaluated according Table 2.2. A setup of the

19 test is shown in Figure 2.11. 
Table 2.2: Typical results of ISAT tests on well cured and oven dried concrete

\begin{tabular}{|c|c|c|c|c|}
\hline \multirow{2}{*}{} & \multicolumn{3}{|c|}{ ISA (ml/m $/ \mathrm{s})$} \\
& \multicolumn{4}{|c|}{ Time after starting the test } \\
\hline & $10 \mathrm{~min}$ & $30 \mathrm{~min}$ & $1 \mathrm{~h}$ & $2 \mathrm{~h}$ \\
\hline High & $>0.50$ & $>0.35$ & $>0.20$ & $>0.15$ \\
\hline Average & $0.25-0.50$ & $0.17-0.35$ & $0.10-0.20$ & $0.07-0.15$ \\
\hline Low & $<0.25$ & $<0.17$ & $<0.10$ & $<0.07$ \\
\hline
\end{tabular}

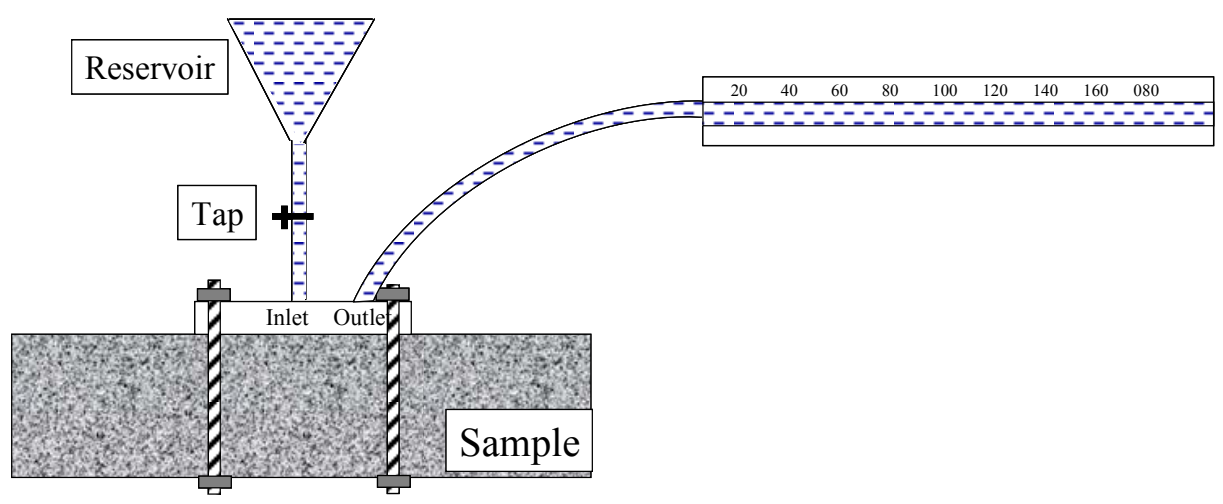

Figure 2.11: Schematic View of the ISAT Method

5 simple nondestructive in situ test method that can be used to measure water penetration

6 into a concrete surface. The difficulty of ensuring a watertight seal is probably one of the

7 greatest limitations of this test because of the problems achieving this in practice.

8 Another limitation is that the measured property is greatly affected by the moisture

9 condition of the concrete. The method cannot be applied to the underside of the concrete 


\section{$1 \quad \underline{2.3 .2 .2 ~ A u t o c l a m}$}

As mentioned before, this method was developed by Basheer et al. (1993). In this

3 system, water goes into the test area through a priming pump with air escaping through

4 the bleed pipe and a pressure transducer measure the water pressure. When the test

5 chamber is completely filled with water the priming pump automatically switches off and

6 the micro pump pressurizes the test area to 0.5 bar above atmospheric, at the stage the test

7 starts. At this pressure the transport of water into capillary pores is considered to be due

8 to absorption rather than by pressure induced flow. As water absorbed by capillary

9 action, the pressure inside would tend to decrease, hence it is maintained constant by

10 pump and the control system. The volume of water delivered is measured and recorded

11 every minute for a test duration of 15 minutes. The quantity of water flowing into the

12 material is plotted against the square root of time. The slope of this graph (which is

13 linear between 5 and 15 minutes) is used to specify a water permeability index with unit

14 of $\mathrm{m}^{3} / \mathrm{min}^{1 / 2}$. Figure 2.12 shows the schematic setup of the test. 


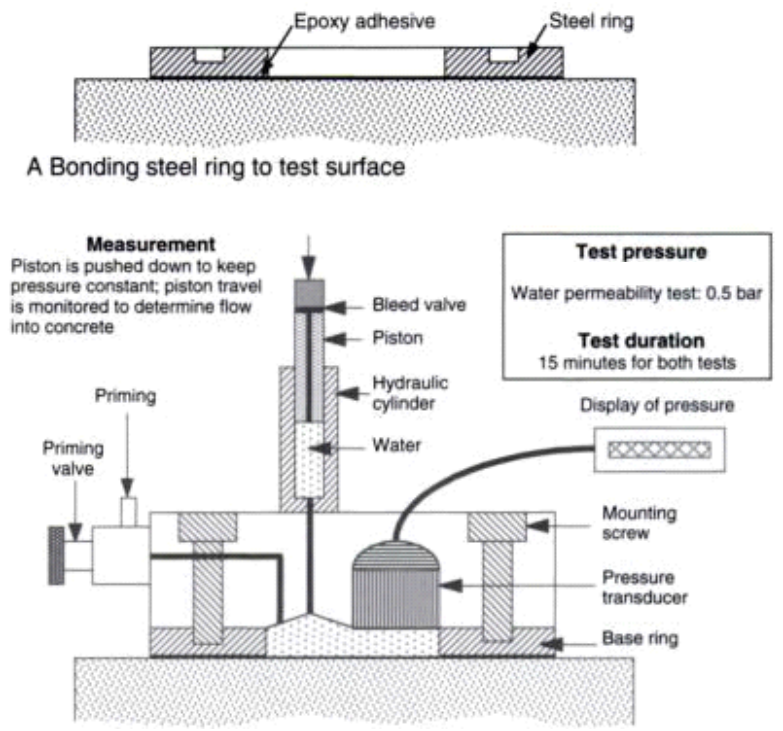

2 Figure 2.12: Schematic Setup of Autoclam Test for Water Permeability Measurement

(Basheer et al. 1993)

\section{$4 \quad$ 2.3.2.3 Germann Water Permeability Test (GWT - 4000)}

The GWT (Germann Water permeability Test) is used for on-site evaluation of:

6 the water permeation of the skin-concrete in finished structure, the water permeation of

7 masonry panels, the water tightness of construction joints and sealed control joints and

8 effectiveness of water proofing membranes (Germann Instruments Inc. 2009).

9 In this method, a pressure chamber containing a watertight gasket is secured

10 tightly to the surface by two anchored clamping pliers or by means of suction plate, (or

11 glue). The chamber is filled with water and the valves closed.

12 The top lid of the chamber is turned until a desired water pressure is achieved.

13 The pressure selected is maintained by means of a micrometer gauge pressing a piston

14 into the chamber, submitting the water penetrating into the material. The travel of the 
1 piston over time is used for characterizing the permeation of the surface tested. Time for

2 test including setting up time is about 40 minutes.

3 The method is somewhat destructive (holes for fixing). Figure 2.13 shows the 4 schematic setup of the test.

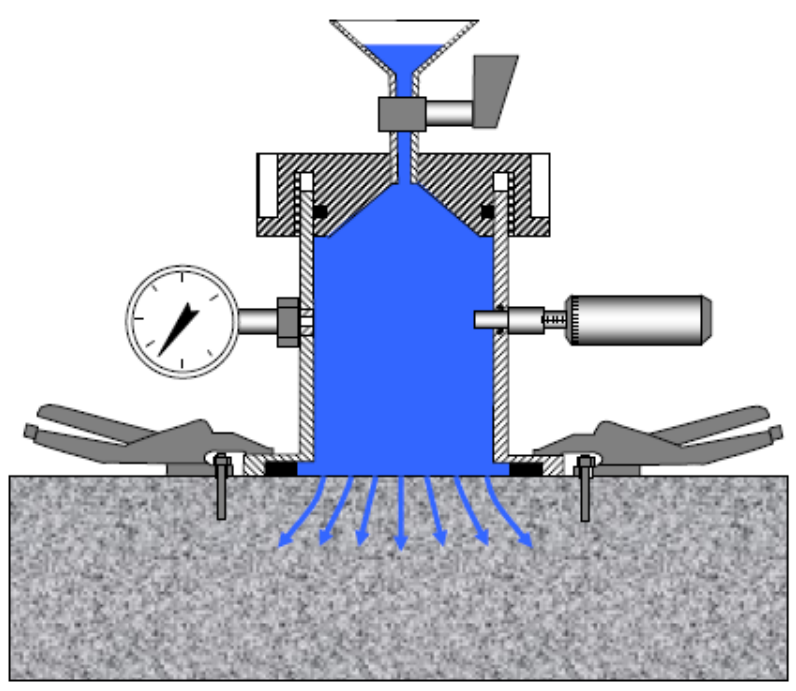

6 Figure 2.13: Schematic Illustration of the GWT Setup (Germann Instruments Inc. 2009)

\section{$7 \quad$ 2.3.2.4 Figg-Poroscope Method}

The development of this method was first reported by J. W. Figg (1973) in

9 England. A schematic showing the apparatus and test setup is presented in Figure 2.14.

10 First, a hole with a diameter of $10 \mathrm{~mm}$ and a depth of $40 \mathrm{~mm}$ is drilled

11 perpendicularly to the surface of the concrete. After clearing, the hole is made airtight by

12 inserting a special silicon rubber plug, which leaves $20 \mathrm{~mm}$ long cylindrical chamber. A

13 hypodermic needle with two concentric tubes is pierced through the rubber after 
1 hardening, and a water head of $100 \mathrm{~mm}$ is applied. The chamber and the capillary tube

2 are filled with water by means of the syringe. After about 1 minute of water contacting

3 the concrete, the stop-cock is closed and the rate of water suction by concrete is

4 monitored by movement of a meniscus in a horizontal capillary. The time taken for

5 concrete to absorb $0.01 \mathrm{ml}$ of water is recorded and reported as the result of the test; and,

6 the results are evaluated based on Table 2.3.

Table 2.3 Concrete Categories Based on the Figg Method

\begin{tabular}{|c|c|c|c|c|c|}
\hline $\begin{array}{c}\text { Concrete } \\
\text { Category }\end{array}$ & 0 & 1 & 2 & 3 & 4 \\
\hline $\begin{array}{c}\text { Protective } \\
\text { quality }\end{array}$ & Bad & Fair & Medium & Good & Very Good \\
\hline $\begin{array}{c}\text { Poroscope } \\
\text { Absorption } \\
\text { Time (s) }\end{array}$ & $<20$ & $20-50$ & $50-100$ & $100-500$ & $>500$ \\
\hline
\end{tabular}

8 Time for test including setting up time is about 40 minutes. The main advantage

9 of this method is that it is simple and easy test. On the other hand, drilling the hole might

10 change the microstructure of the concrete. 


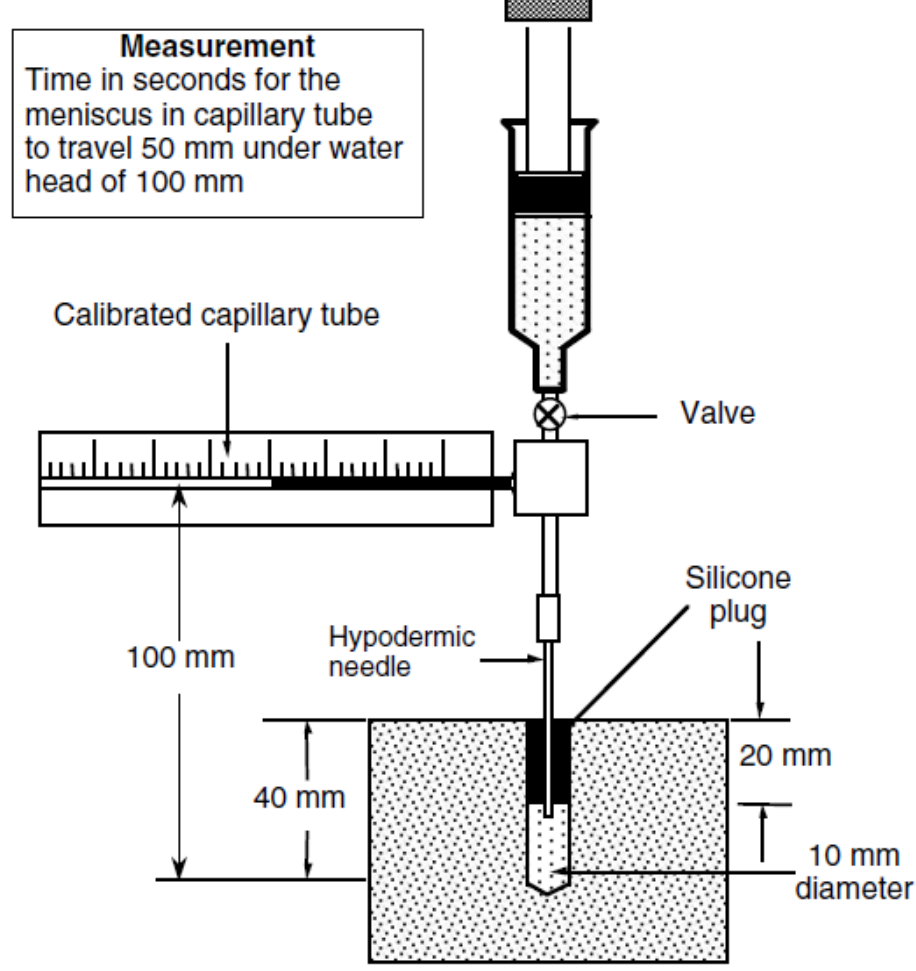

$3 \quad$ 2.3.2.5 Field Permeability Test (Florida Test) - FPT

4 This method is developed by Developed by Meletiou, Tia and Bloomquist in

5 1990s (Meletiou et al. 1992; Meletiou et al. 1993). It starts with drilling a hole (23 mm in

6 diameter and $152 \mathrm{~mm}$ deep into the concrete. Then a cylindrical probe is introduced into

7 the hole. Tightening the top nut causes the two neoprene packers to extend and seal a

8 central chamber. A full vacuum is applied to the chamber for 5 to 10 minutes. The probe

9 is then connected to the instrumentation unit. Water, pressurized by nitrogen bottle, is

10 introduced into the chamber through the hollow probe, at the pressure within the range of

1110 to 35 bar (normally 17 bar) and follows radically into the surrounding concrete. After

12 steady-state flow is achieved, the flow rate of the water is recorded (mean value pf the 5 
1 measurements) and the coefficient of water permeability is calculated according to

2 Darcy's law. Time for the test including the setting up time is about 40 minutes and it is

3 slightly destructive due to drilling the hole Figure 2.15 shows the schematic setup of the

4 test.

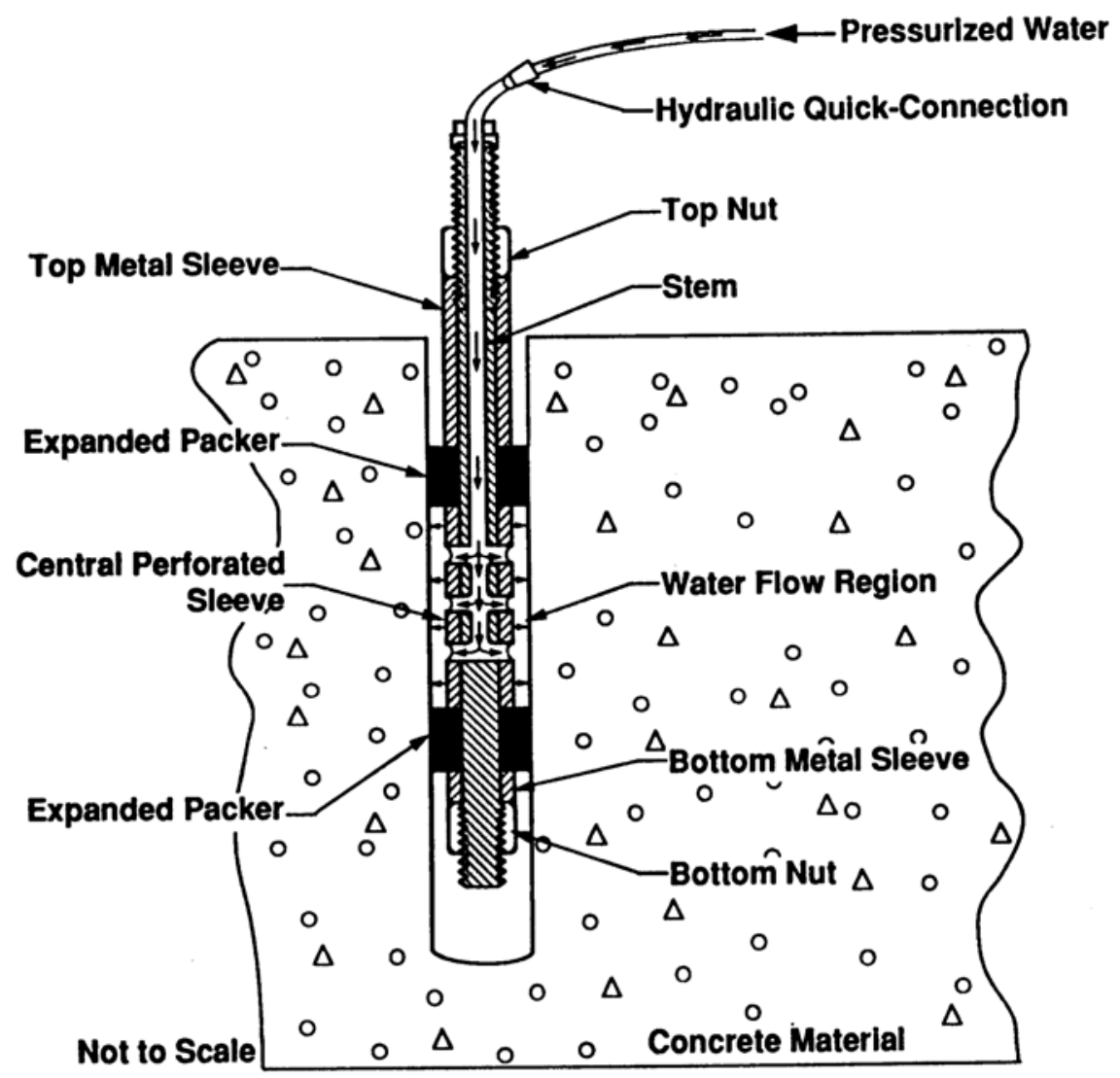

Figure 2.15: Schematic View of the Florida Test Setup 


\subsubsection{Migration}

2 Ion migration in concrete is a complex process which involves: diffusion, 3 capillary suction, convective flow with flowing water and electrical field. Followings are

4 some of the methods for monitoring/measuring the ion migration in concrete.

\section{$5 \quad$ 2.3.3.1 Electrical Resistivity Methods}

6 In concrete, electrical current is carried out by ions dissolved in the pore solution.

7 More pore solution, as well as more and larger pores with higher degree of connectivity

8 cause lower resistivity and this can be used as the indication of concrete permeability.

$9 \quad$ a) Resistivity - 4 point (Wenner) Method

10 The four-probe method is one of the most widely used technique for field 11 measurement of concrete resistivity. This method was originally developed by F. Wenner 12 (1996) to measure the resistivity of soil. In this technique, four electrodes are equally

13 spaced (As shown in Figure 2.16) and a small alternating current is applied between the

14 outer electrodes while potential is measured between the inner electrodes. The resistivity 15 is then calculated by using the Equation 2.14:

$16 \rho=\frac{2 \pi a V}{I}$ 
1 where $\rho$ is the resistivity $(\Omega . \mathrm{cm})$, a is the distance between inner electrodes $(\mathrm{cm})$ and $\mathrm{V}$

2 and I are maximum values of voltage (volts) and current (amps), respectively. Factors

3 that may affect the results of four probe technique measurement are:

4 1. geometrical constraints,

5 2. surface contact,

6

3. concrete non-homogeneity,

4. the presence of steel reinforcing bars,

5. surface layers having different resistivity from the bulk of the concrete, and

6. ambient temperature.

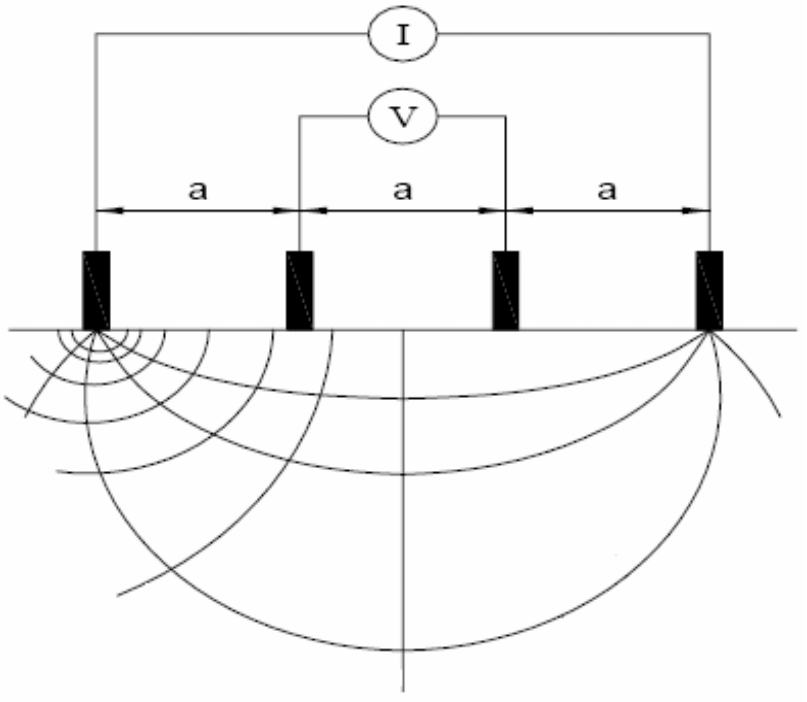

Figure 2.16: Schematic Illustration of the 4 Point (Wenner) Method for Resistivity Measurement 
b) Resistivity - Disk Method

2 It consists in placing one metal electrode on the concrete surface and measuring

3 the resistance between this electrode and the reinforcement or placing concrete between

4 two metal electrodes and measuring the concrete resistance (shown in Figure 2.17). This

5 technique was introduced in 1988 for filed application (Newton and Sykes 1988). In this

6 method, a short-time anodic current pulse is applied galvanostatically between metal

7 electrode placed on the concrete surface and the rebar (or between two metal electrodes).

8 The applied current is usually in the range of 10 to $100 \mu \mathrm{A}$ and the typical pulse duration

9 is between 5 to 30 seconds. The plot of resultant potential difference versus time can be

10 used to determine the concrete resistance as shown in Figure 2.18.
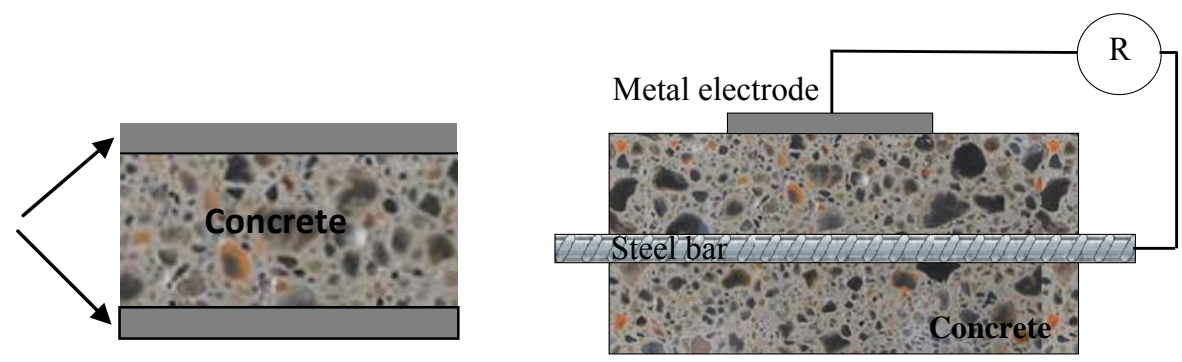


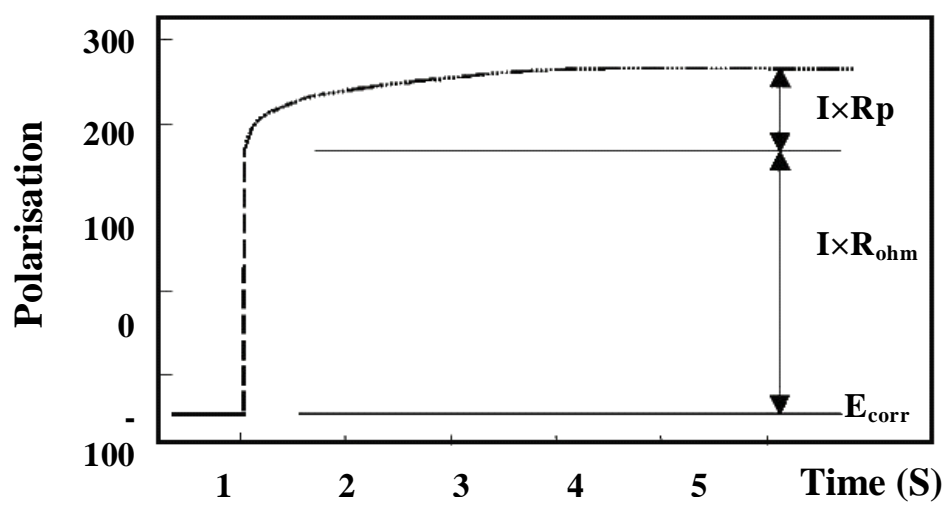

2 Figure 2.18: Potential Response of the Galvanostatic Pulse Technique. $R_{\mathrm{ohm}}$, Represents 3 Concrete Resistance,

c) Electrical Impedance Spectroscopy (EIS)

5 EIS studies the system response to the application of a small amplitude alternating

6 potential signal at different frequencies. If, at each excitation frequency, the real part is

7 plotted on the $\mathrm{x}$-axis and the imaginary part is plotted on the $\mathrm{y}$-axis of a chart, a "Nyquist

8 plot" is formed. From this plot, the resistance of the bulk material can be determined

9 (Figure 2.19). 


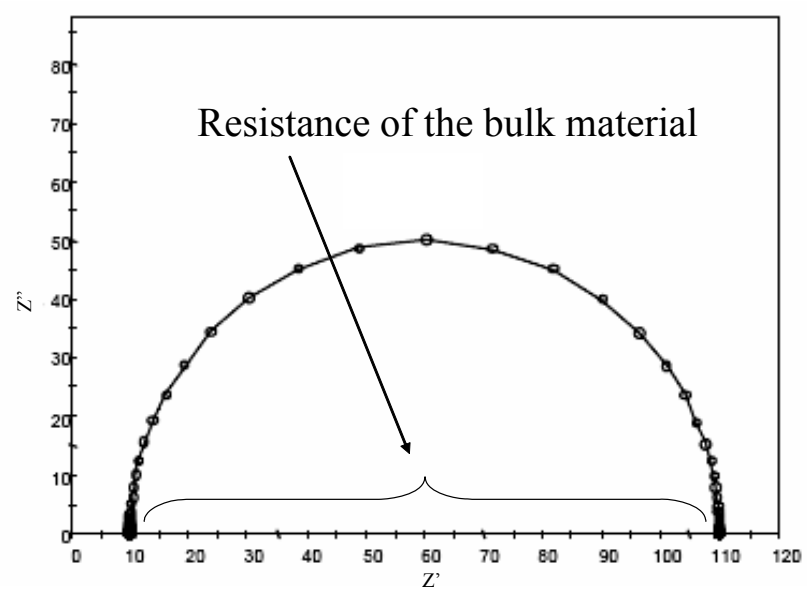

5 solution, by applying electrical potential between the source of ion and the concrete. The

6 movement of ions, due to the action of the electrical field is called migration. By

7 applying electrical field to the concrete through two electrodes, several processes 8 develop:

- In both anode and cathode, gases may be generated.

- The ions move through the pore solution in the direction of the electrode of opposite charge. 
e) ASTM C1202 and AASHTO T277

The test method involves obtaining a $100 \mathrm{~mm}$ diameter core or cylinder sample

3 from the concrete being tested (Figure 2.20). A $50 \mathrm{~mm}$ specimen is cut from the sample.

4 The side of the cylindrical specimen is coated with epoxy, and after the epoxy is dried, it

5 is put in a vacuum chamber for 3 hours. The specimen is vacuum saturated for 1 hour

6 and allowed to soak for 18 hours. It is then placed in the test device. The left-hand side

7 (-) of the test cell is filled with a $3 \% \mathrm{NaCl}$ solution and the right-hand side $(+)$ of the test

8 cell is filled with $0.3 \mathrm{~N} \mathrm{NaOH}$ solution. The system is then connected and a 60 -volt

9 potential is applied for 6 hours. Readings are taken every 30 minutes. At the end of 6

10 hours the sample is removed from the cell and the amount of coulombs passed through

11 the specimen is calculated and the results are interpreted based on Table 2.4.

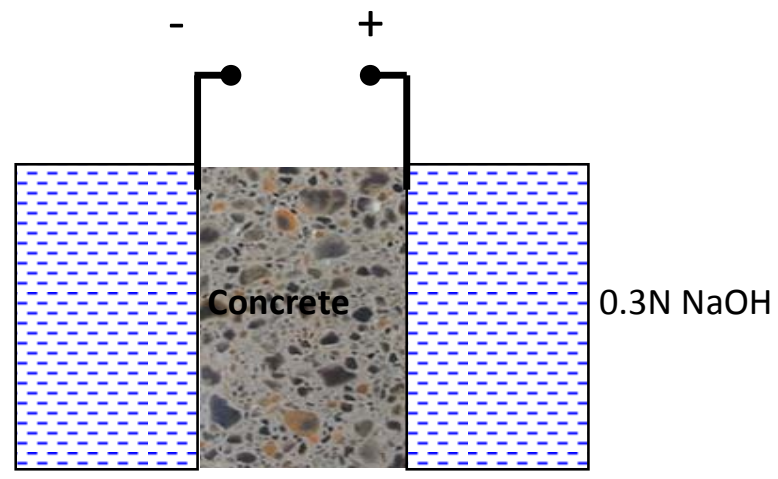


Table 2.4: Interpretation of the ASTM C1202 Test (RCP)

\begin{tabular}{|c|c|c|}
\hline $\begin{array}{c}\text { Charge Passed } \\
\text { (Coulombs) }\end{array}$ & Chloride Permeability & Typical of \\
\hline$>4000$ & High & $\begin{array}{c}\text { High W/C ratio }(>0.60) \\
\text { conventional PCC }\end{array}$ \\
\hline $2000-4000$ & Moderate & $\begin{array}{c}\text { Moderate W/C ratio }(0.40-0.50) \\
\text { conventional PCC }\end{array}$ \\
\hline $1000-2000$ & Low & $\begin{array}{c}\text { Low W/C ratio }(<0.40) \\
\text { conventional PCC }\end{array}$ \\
\hline $100-1000$ & Very low & $\begin{array}{c}\text { Latex-modified concrete or } \\
\text { internally-sealed concrete }\end{array}$ \\
\hline
\end{tabular}

There are many factors that may affect the accuracy of the test procedure. The

3 age and curing of the test specimen affects the results dramatically. In general, the older

4 the specimen, the lower the coulombs, assuming that the sample has been cured properly.

5 Research has also indicated that the presence in the concrete of admixtures containing

6 ionic salts may affect the results obtained. Cement factor, air content, water/cement ratio,

7 curing of the test sample, aggregate source or type are the other factors that can affect the 8 test results.

9 This test method does not replicate actual conditions that concrete would

10 experience in the field. There is no condition where concrete is exposed to a 60 -volt

11 potential. This test method does not measure concrete permeability. What it does

12 measure is concrete resistance to electrical current. Resistance is calculated as volts

13 divided by current. It has been shown that there is a fair correlation between concrete

14 resistivity and concrete permeability. 
f) AASHTO T259

The test requires three slabs at least $75 \mathrm{~mm}$ thick and having a surface area of 300

$3 \mathrm{~mm}$ square. These slabs are moist cured for 14 days then stored in a drying room at 50

4 percent relative humidity for 28 days. The sides of the slabs are sealed but the bottom

5 and top face are not (Figure 2.21). After the conditioning period, a $3 \% \mathrm{NaCl}$ solution is

6 ponded on the top surface for 90 days, while the bottom face is left exposed to the drying

7 environment. At the end of this time the slabs are removed from the drying environment

8 and the chloride concentration of 0.5 -inch thick slices is then determined.

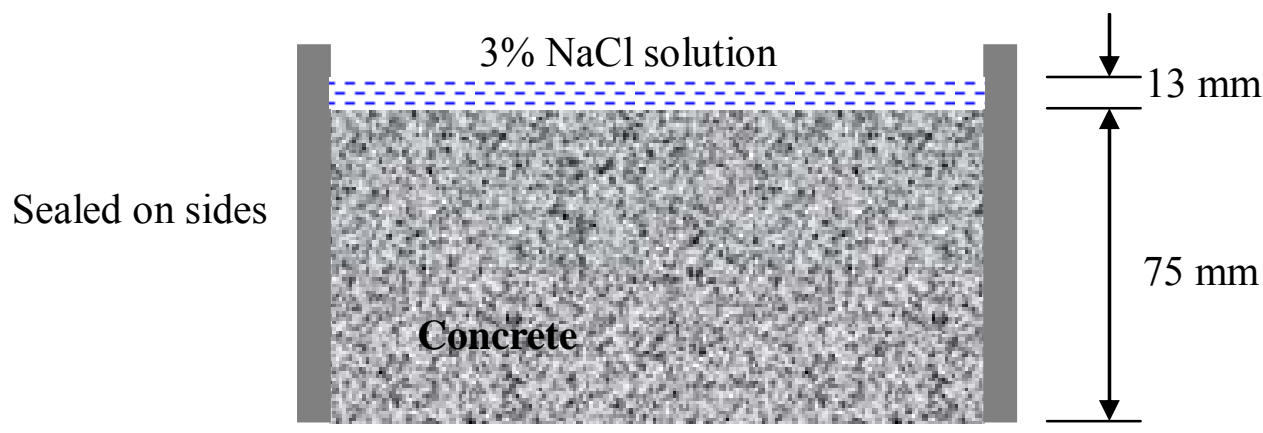

$50 \% \mathrm{RH}$

9

10

11

Limitations of this method can be summarized as:

Figure 2.21: AASHTO T259 Test Setup

- Little information is being gathered about the chloride profile.

- The salt ponding test does provide a crude one-dimensional chloride ingress profile, but this profile is not just a function of chloride diffusion. Since the 
9 the salt ponding test (AASHTO T257) to measure diffusion. In this method, the test

10 specimen is saturated with limewater. This prevents any initial sorption effects. Also,

11 instead of coating just the sides of the sample and leaving one face exposed to air, the

12 only face left uncovered is the one exposed to a $2.8 \mathrm{M} \mathrm{NaCl}$ solution. It is left this way

13 for a minimum of 35 days before evaluation. To evaluate the sample, the chloride profile

14 of the concrete is determined at depth increments on the order of $0.5 \mathrm{~mm}$. The chloride

15 content of the powder is then determined according to AASHTO T260 (Figure 2.22). 
4 still a long-term test. For low quality concretes, the minimum exposure period is 35 days.

5 For higher quality concretes, however, this period must be extended to 90 days or longer, 6 just as for the salt ponding test.

10 voltage of $30 \mathrm{~V}$. The experiment proceeds as usual for an electrical migration test, except

11 that the chloride concentration of the downstream solution is not monitored. Instead,

12 after a specified duration ( $\sim 8 \mathrm{hrs}$.) the samples are removed and split, and the depth of

13 chloride penetration is determined in one half of the specimen using a $0.1 \mathrm{~N}$ silver nitrate

14 solution as a colorimetric indicator. The color change border corresponds to the location

15 of a soluble chloride concentration of $0.15 \%$ by weight of cement (Otsuki et al. 1992). 


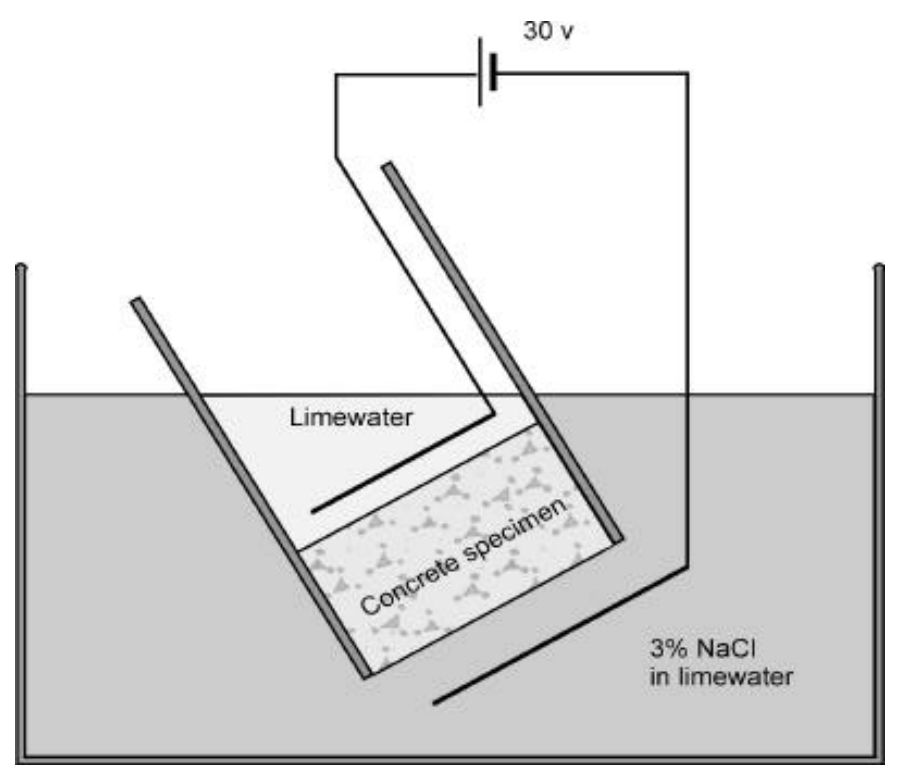

\section{$3 \quad$ 2.3.3.2 TDR (Time-domain Reflectometry) (Cerny 2009)}

4 The TDR method is a dielectric method, based on analysis of the behavior of

5 dielectrics in time-varying electric field and consists in the measurement of permittivity

6 of moist porous media. The determination of moisture content using the permittivity

7 measurements is based on the fact the static relative permittivity of pure water is equal to

$8 \sim 80$ at $20^{\circ} \mathrm{C}$, while for most dry building materials it ranges from 2 to 6 . The principle

9 of TDR device consists in launching of electromagnetic waves together with the time

10 intervals between launching the waves and detecting the reflections. A TDR system for

11 field measurement generally includes a TDR device, which consists of pulse generator

12 and sampler, a connection cable, and a measurement probe. The pulse generator sends

13 out pulse (which generally is a fast rising step pulse) and the sampler records the

14 response from the system. 
2 A literature review of transport properties affecting durability of concrete and a 3 revision of the test methods available to measure permeability had been performed in this

4 chapter. The main equations that describe the theory of fluid transport were presented.

5 The effects of water to cement ratio, curing, and supplementary materials such as silica

6 fume, slag, and fly ash were also described. It was shown that the effect of sample

7 preparation is a paramount issue when absorption and gas permeability need to be

8 measured. Samples prepared under different conditions will have different relative

9 humidity or different moisture content. This can lead to a misleading test result, perhaps

10 more reflecting sample preparation method than the actual porosity network.

11 An exhaustive revision of the available test methods to measure permeability was

12 performed. Table 2.5 shows a summary of the techniques described in this chapter. 


\begin{tabular}{|c|c|c|c|c|c|c|c|c|c|}
\hline 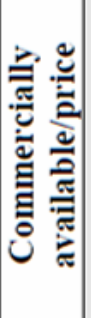 & & ஜ̊ & 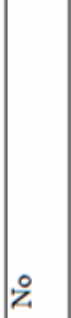 & 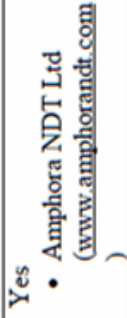 & 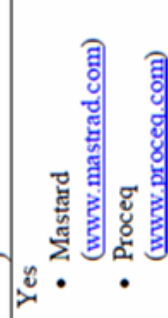 & $\frac{1}{2}$ & $\stackrel{2}{z}$ & 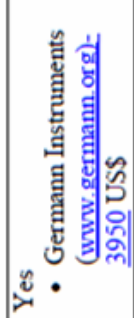 & 을 \\
\hline 总 & & 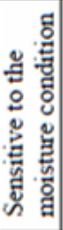 & 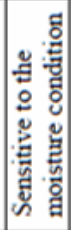 & 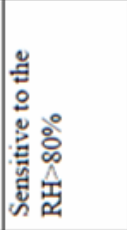 & 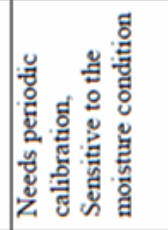 & & & 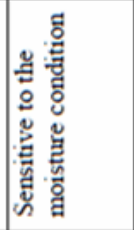 & \\
\hline 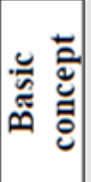 & & 悬 & 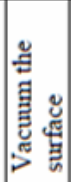 & 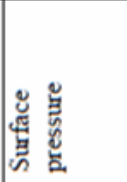 & 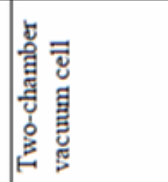 & 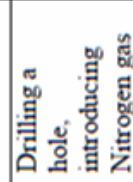 & 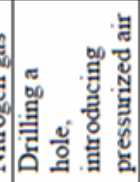 & 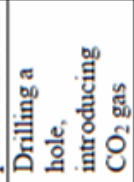 & 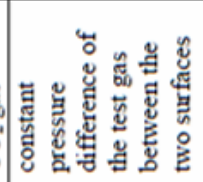 \\
\hline 窝 & & $N$ & $N$ & - & - & $N$ & $N$ & $\sim$ & $N$ \\
\hline : & & 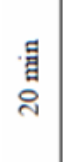 & . & 見 & $\begin{array}{l}\text { 見 } \\
\text { 尺े }\end{array}$ & $\begin{array}{l}\text { 見 } \\
8\end{array}$ & $\begin{array}{l}\overrightarrow{1} \\
\text { 自焉 } \\
8\end{array}$ & $\begin{array}{l}\text { 見 } \\
\text { 古 }\end{array}$ & 笶 \\
\hline 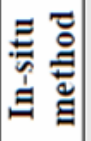 & & $x$ & $x$ & $x$ & $x$ & $x$ & $x$ & $x$ & \\
\hline 을 & & $x$ & $x$ & $x$ & $x$ & $x$ & $x$ & $x$ & $x$ \\
\hline 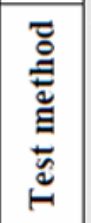 & 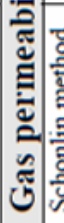 & 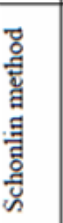 & 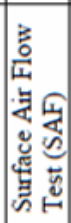 & & 总 & 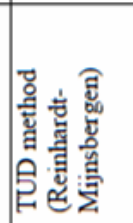 & 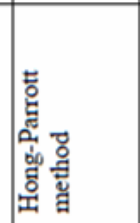 & 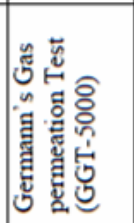 & 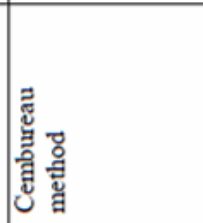 \\
\hline
\end{tabular}




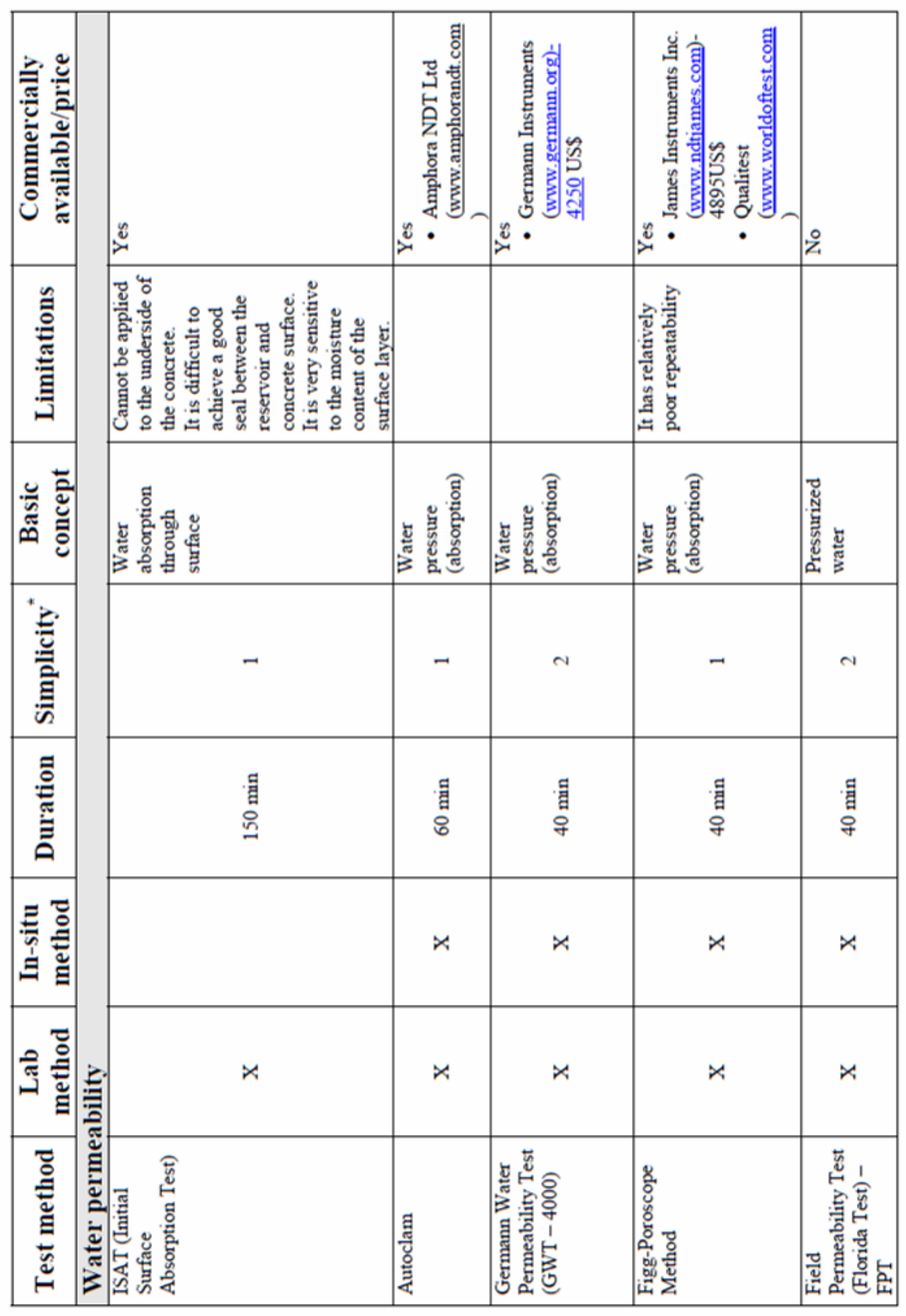




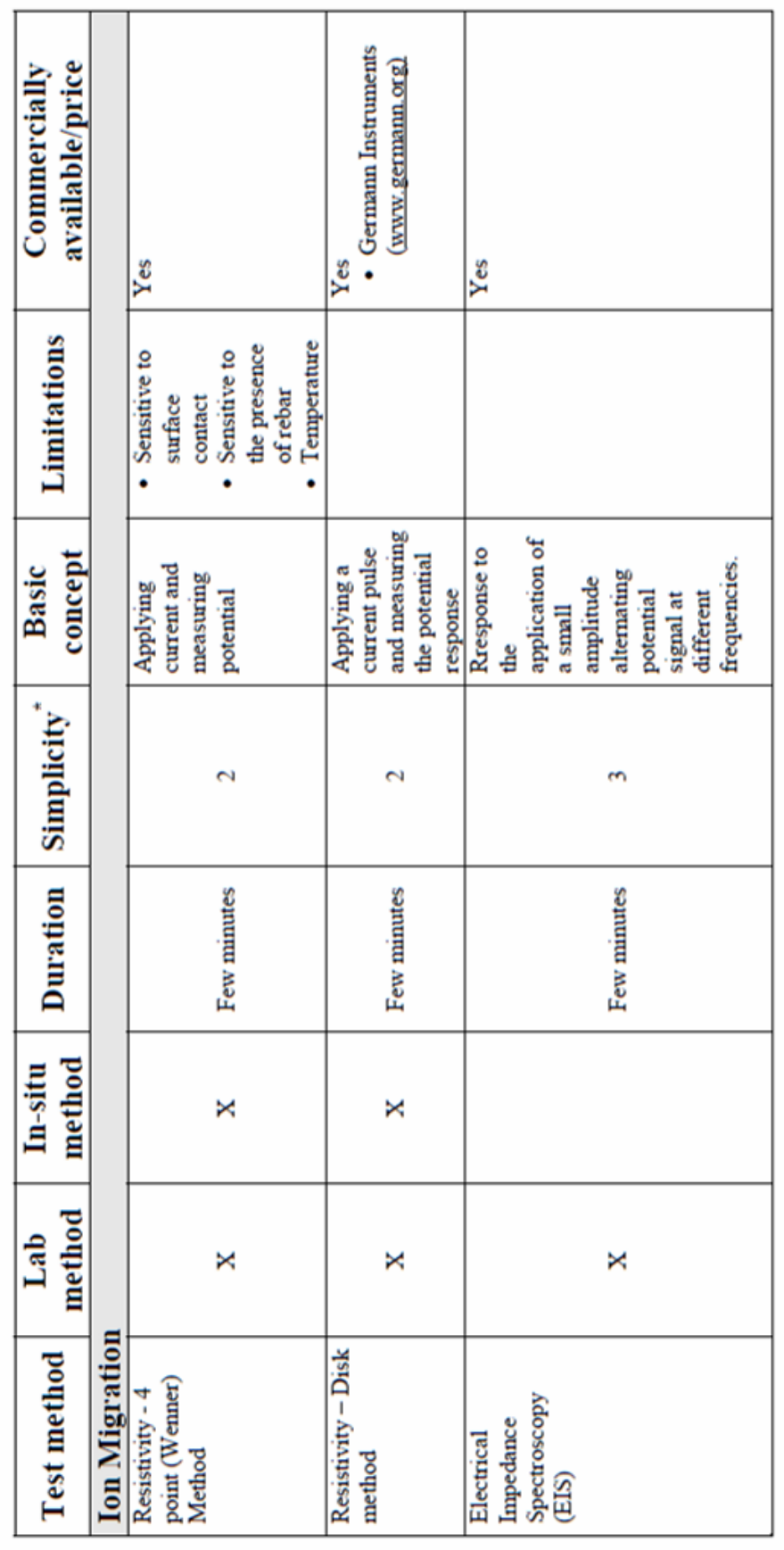




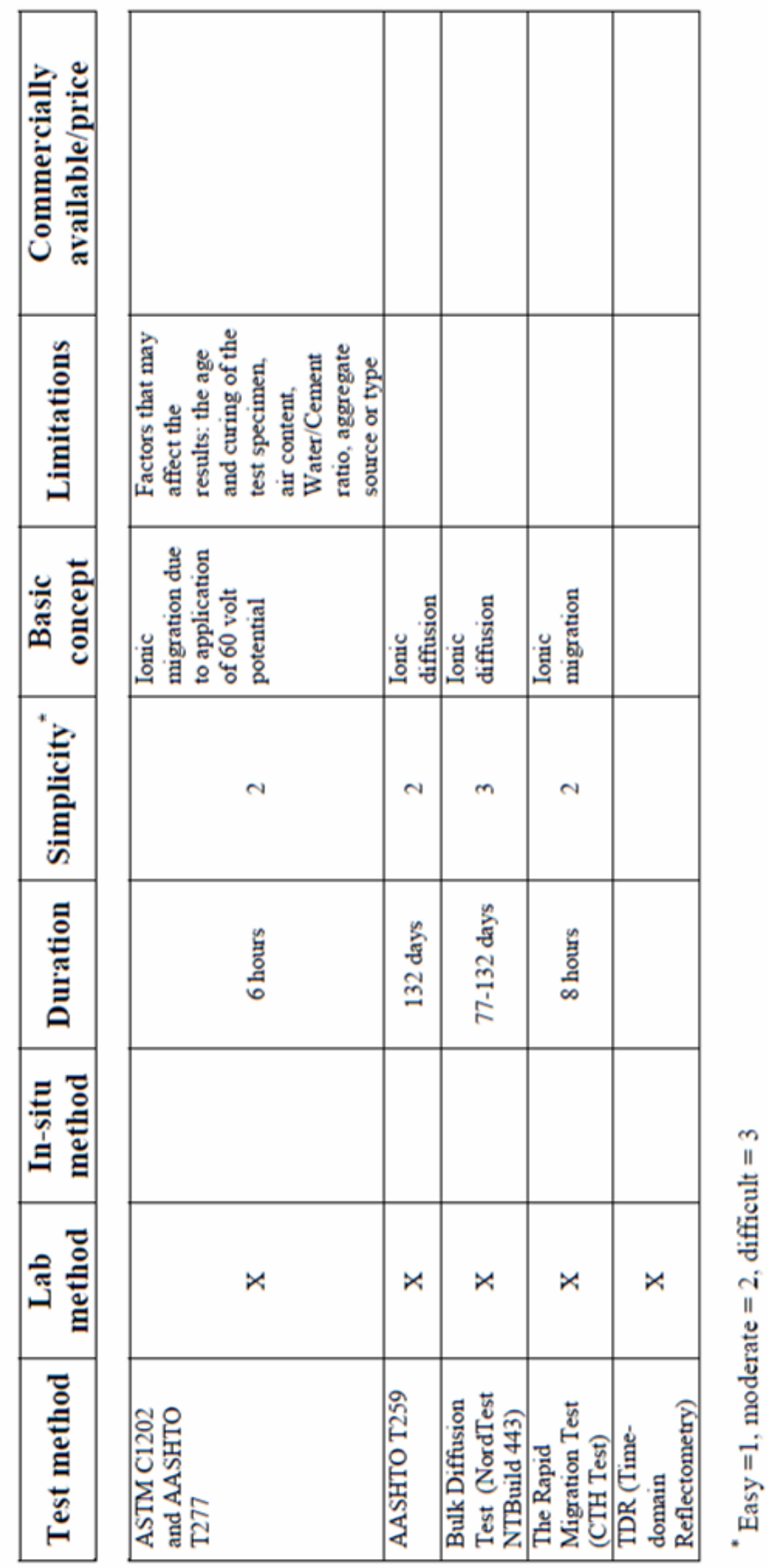




\section{$\underline{3.1 \text { Introduction }}$}

4 This chapter describes the main transport characteristics of the samples collected

5 from the field. Originally four concrete mixtures were considered to be evaluated,

6 however a total of fourteen different concrete were analyzed, representing a range of

7 mixtures proportions that are typical across the state.

$9 \quad$ Fourteen different mixtures were collected from the field. Table 3.1 shows a detail

10 of the project selected by the SAC. Table 3.1 includes mixture proportions and the age of

11 the concrete at the time they were received for preparation. 
Table 3.1 Characteristics of Concrete Sample Collected from the State of Indiana

\begin{tabular}{|c|c|c|c|c|c|c|c|c|c|c|c|}
\hline Designation & Project & $w / c$ & $\begin{array}{c}\text { Cement } \\
\mathrm{lb} / \mathrm{ft}^{3}\end{array}$ & $\begin{array}{l}\text { Fly Ash } \\
\mathrm{lb} / \mathrm{ft}^{3}\end{array}$ & $\begin{array}{c}\text { S. Fume } \\
\mathrm{lb} / \mathrm{ft}^{3}\end{array}$ & $\begin{array}{l}\text { Slag } \\
\mathrm{lb} / \mathrm{ft}^{3}\end{array}$ & $\begin{array}{l}\text { Total } \\
\mathrm{lb} / \mathrm{ft}^{3}\end{array}$ & $\begin{array}{c}\text { Paste Vol } \\
\% \\
\end{array}$ & \begin{tabular}{|c|} 
Air Content \\
$\%$ \\
\end{tabular} & Agg & Age of Samples \\
\hline Concrete \#1 & E, LaPorte & 0.40 & 539 & - & - & - & 539 & $29.5 \%$ & NA & Satndard & 2 yr. (core) \\
\hline Concrete \#2 & F, Seymour & 0.39 & 540 & - & - & - & 540 & $29.2 \%$ & NA & Satndard & 2 yr. (core) \\
\hline Concrete \#3 & LaPorte & 0.43 & 590 & - & - & - & 590 & $32.8 \%$ & NA & Slag & 2.5 months \\
\hline Concrete \#4 & C, LaPorte & 0.39 & 590 & - & - & - & 590 & $31.3 \%$ & 7.2 & Slag & 4 yr. (core) \\
\hline Concrete \#5 & Rockville Road & 0.42 & 400 & 110 & - & - & 510 & $29.2 \%$ & 7.0 & Satndard & $2 \mathrm{mo}$. \\
\hline Concrete \#6 & Airport, P-501 & 0.39 & 449 & 149 & - & - & 598 & $31.6 \%$ & 5.5 & Satndard & $1.5 \mathrm{yr}$ \\
\hline Concrete \#7 & IMI Bridge deck & 0.40 & 533 & 101 & - & - & 634 & $34.0 \%$ & NA & Satndard & $1.5 \mathrm{yr}$ \\
\hline Concrete \#8 & SR 267 & 0.42 & 440 & 70 & - & - & 510 & $29.2 \%$ & 5.5 & Satndard & $1.5 \mathrm{yr}$ \\
\hline Concrete \#9 & D, LaPorte & 0.42 & 440 & 100 & - & - & 540 & $30.6 \%$ & NA & Satndard & 2 yr. (core) \\
\hline Concrete \#10 & IB & 0.42 & 490 & 125 & - & - & 615 & $34.0 \%$ & NA & Slag & 2.5 months \\
\hline Concrete \#11 & A, LaPorte & 0.38 & 480 & 110 & - & - & 590 & $31.2 \%$ & 6.4 & Slag & 6 yr. (core) \\
\hline Concrete \#12 & IR-29137 & 0.39 & 445 & 125 & 32 & - & 602 & $32.9 \%$ & 6.5 & Satndard & 1 month (core) \\
\hline Concrete \#13 & IB-29153 & 0.39 & 445 & 125 & 32 & - & 602 & $32.9 \%$ & 6.5 & Satndard & 1 month (core) \\
\hline Concrete \#14 & B, LaPorte & 0.41 & 440 & - & - & 120 & 560 & $30.2 \%$ & 6.2 & Slag & 1 yr. (core) \\
\hline
\end{tabular}

From Table 3.1 samples can be separated in six groups considering the binder

4 material and the type of aggregate (standard or slag aggregate). These groups are:

a) Concrete \#1 and \#2 : Plain cement, with standard aggregate

b) Concrete \#3 and \#4 : Plain cement, with slag aggregate

c) Concrete \#5 to \#9 : Plain cement plus fly ash, with standard aggregate

d) Concrete \#10 and \#11: Plain cement plus fly ash, with slag aggregate

e) Concrete \#12 and \#13: Plain cement plus silica fume, with standard aggregate

1

f) Concrete \#14 : Plain cement plus slag cement, with slag aggregate

13 Samples were tested under several different tests: volume of air voids, volume of

14 permeable voids, water absorption, diffusivity, moisture absorption-desorption, and 
1 electrical conductivity. Tests technique and results are shown separately for each test.

2 Electrical conductivity results are analyzed separately in Chapter 7.

5 susceptibility of the cement paste portion of the concrete to damage by freezing and

6 thawing. ASTM C457 (ASTM 2009) had been commonly used to quantify the air

7 content system. The procedure consists of the determination of the volumetric

8 composition of the concrete by observation of the frequency with which areas of a given

9 component coincide with a regular grid system of points at which stops are made to

10 enable the determinations of composition. The data gathered are the linear distance

11 between stops along the traverse (I), the total number of stops ( $\mathrm{St}$ ), the number of stops in

12 air voids $(\mathrm{Sa})$, the number of stops in paste $(\mathrm{Sp})$, and the number of air voids $(\mathrm{N})$

13 intersected by the line of traverse over which the component data is gathered. From these

14 data the air content and various parameters of the air-void system are calculated. If only

15 the air content is desired, only Sa and St need be determined.

16 Although ASTM C457 does not currently recognize any automated methods, the

17 field of concrete research has long recognized the fact that it is difficult to find people

18 willing to measure large numbers of air-voids, and thus several automated methods have

19 been developed. The earliest automated procedure was described by Chatterji (1976) and

20 relied on a sample preparation contrast enhancement step to make air-voids appear white

21 and to make aggregates and paste appear black. This principle is still widely used today

22 by and a more recently developed method was used in this work (Peterson 2008). 
Sample preparation start by lapping the specimen with successively finer

2 abrasives until it is suitable for microscopical observation. After the samples are polished,

3 black and white contrast may be obtained by painting the surface of the polished sample

4 with a wide tipped black permanent marker. Normally three coats are used, changing the

5 orientation $90^{\circ}$ between coats. After the ink is dry, the voids are filled with white zinc

6 powder (median size smaller that $2 \mu \mathrm{m}$ ). Powder is worked into the samples using the flat

7 face of a glass slide. A razor blade is used to scrape away excess powder, leaving behind

8 powder pressed into voids. Residual powder is removed by wiping with a clean and

9 lightly oiled fingertip. A fine tipped black marker can be used to darken voids in

10 aggregates (Peterson 2008). Figure 3.1 shows pictures of a sample prepared to be

11 scanned. 

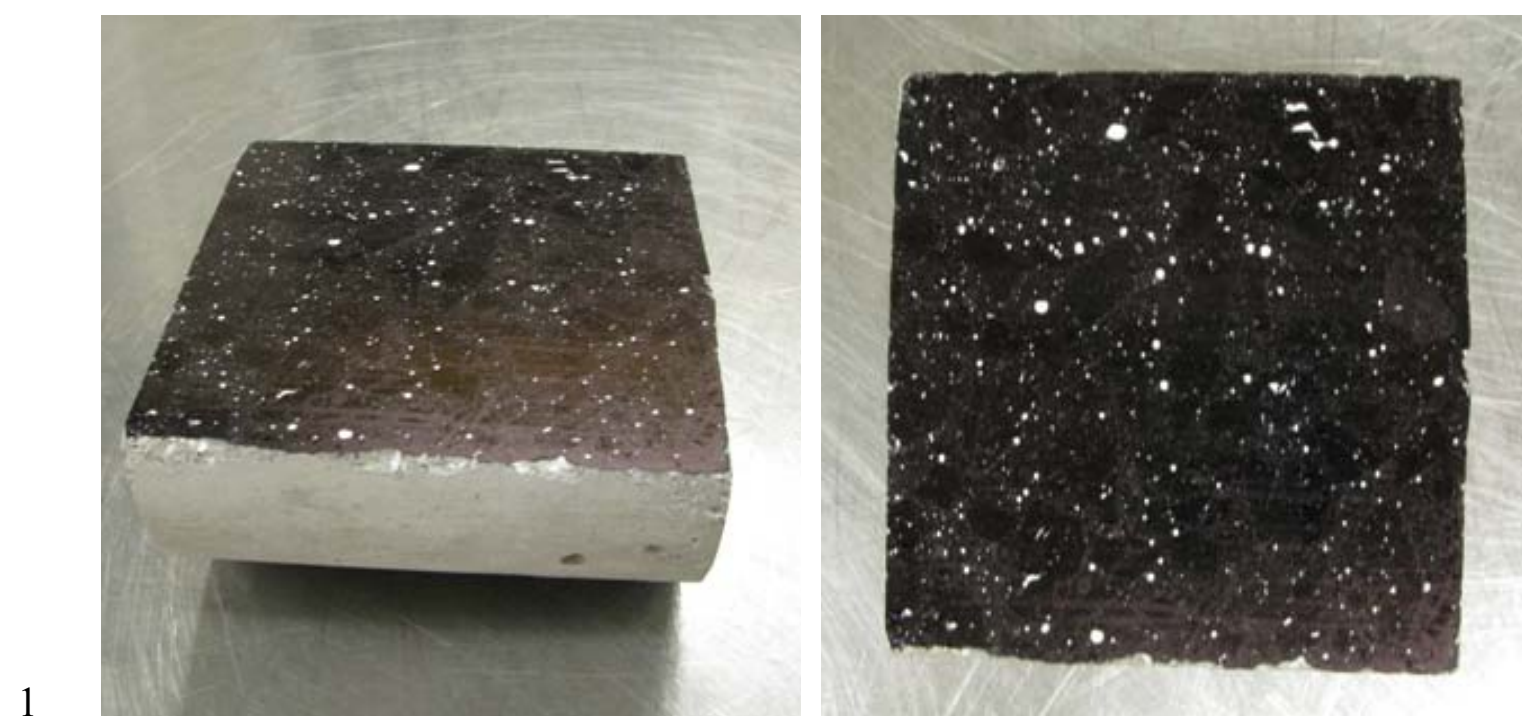

Figure 3.1: Prepared Samples for Air Void Count, Automated Method (Sample Dimension: $4 \mathrm{x} 4$ inches)

5 scanner. The first step in the analysis of a digital image is to classify the pixels that

6 represent the air-voids. Most segmentation procedures based on black and white contrast

7 enhancement begin with a choice of threshold. Pixels in the digital image darker than the

8 threshold level are classified as non-air, pixels brighter than the threshold level are

9 classified as air. Many of these procedures employ additional digital processing to further

10 refine the distinction between air and non-air pixels (Peterson 2008). For this study, the

11 air void content will be quantified using an automated procedure.

12 Figure 3.2a shows the results from air void content, measured using an automated

13 method. Due to difficulties in segmenting voids space in the slag aggregate, only

14 samples containing standard aggregate were considered (total of nine concrete samples).

15 The results show that concrete samples typically used in Indiana had an entrained air

16 volume between $3 \%$ and $5 \%$ of the total concrete volume. It should be noted that has 
1 been recommend (Hover 1977) that a minimum of $18 \%$ of the cement paste was

2 entrained air as a method to prevent damage from the freezing and thawing. This

3 percentage can be calculated from Figure 3.2a dividing the percentage of entrained air by

4 the cement paste proportion (Figure 3.2b). It can be observed that none of these mixtures

5 reach the recommended $18 \%$ of entrained air expressed as a function of the paste

6 volume.

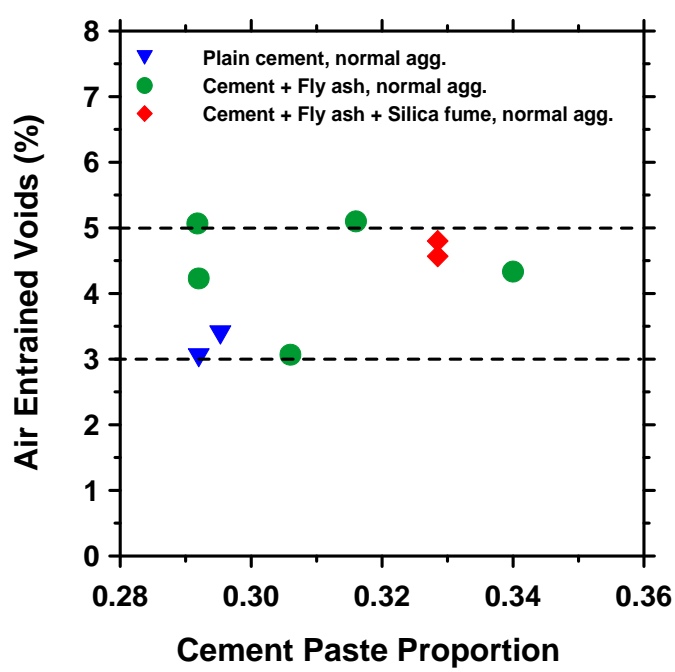

a)

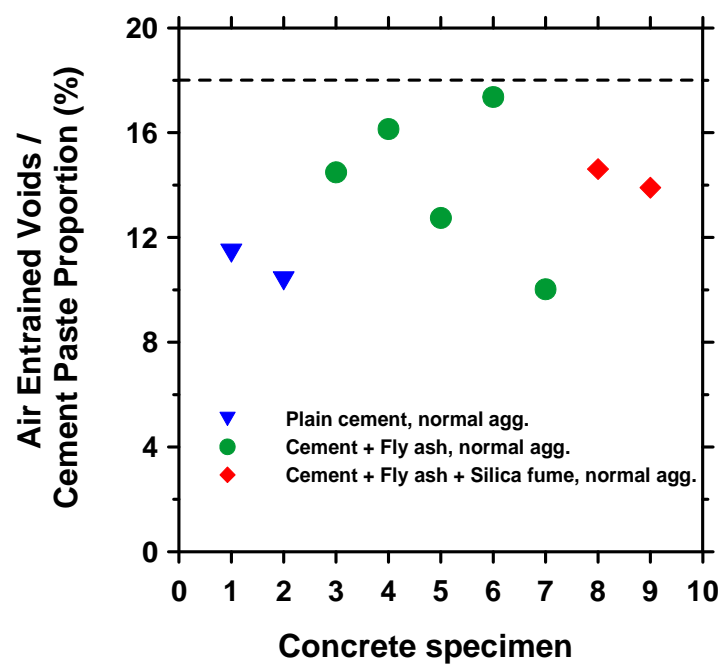

b)

Figure 3.2: Results for Entrained Air

ASTM C-642 is the standard method used to calculate density, percent absorption

10 and total percent voids in hardened concrete. The method consists of determining the

11 total volume of pores comparing the oven dry mass, the saturated mass after immersion,

12 the saturated mass after boiling or vacuum, and the immersed apparent mass. These 
1 parameters allow calculating the volume of the sample and then the total percentage of

2 void on the concrete, including gel pores, capillary pores and air entrained pores.

3 Figure 3.3 shows the total air content for the fourteen mixtures. The results show

4 that standard pavement concrete in Indiana contains a total pore volume in the range of

$511.0 \%$ to $14.5 \%$ of the total concrete volume. This plot also shows the influence of the

6 porosity of the slag aggregate on the test. Sample containing just standard aggregate have

7 total porosity in the range of $11 \%$ to $12 \%$.

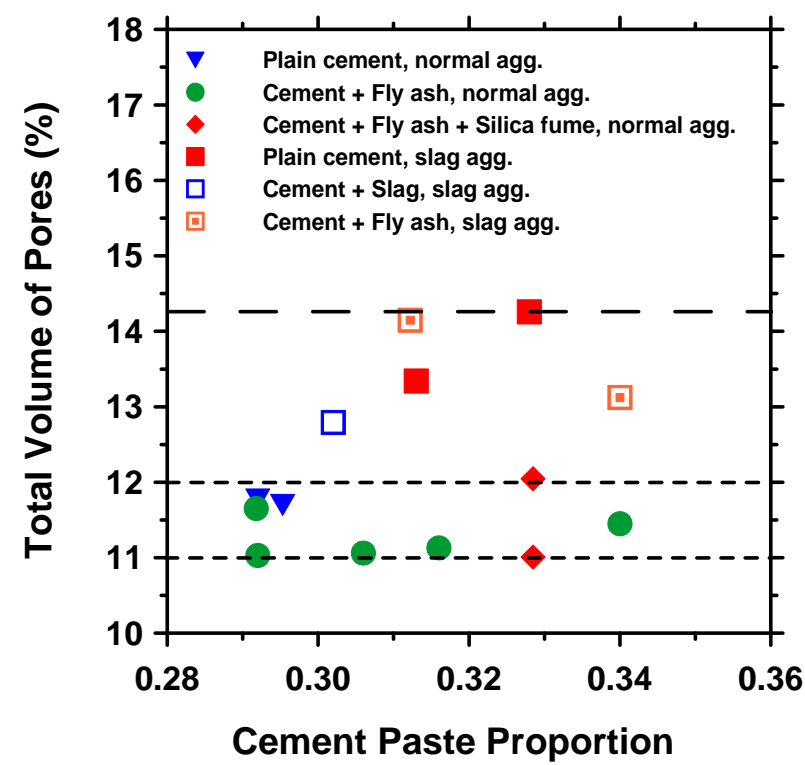


The durability of concrete subjected to aggressive environments depends largely

4 on the penetrability of the pore system (ASTM 2004, Sabir et al. 1998, Maltais et al.

5 2004, Hooton et al. 1993, Parrot 1992, Fagerlund 1996). Three mechanisms can be used

6 to describe transport in cementitious systems: permeability, diffusion and absorption.

7 Permeability is the measure of the flow of water under a pressure gradient, while

8 diffusion is the movement of ions due to a concentration gradient. Absorption can be

9 described as the materials ability to take in water by means of capillary suction. Water

10 absorption is an important factor for quantifying the durability of cementitious systems

11 (Parrot 1992, Hooton et al. 1993, Fagerlund 1996, Bentz et al. 2001, Yang 2006,

12 Henkensiefken et al. 2009) and is the primary focus in this section.

13 ASTM C1585 (ASTM 2004) is commonly used to determine the absorption and

14 rate of absorption (sorptivity) of water in unsaturated hydraulic cement concretes. This

15 test method, based on work reviewed by Hall (1989), consists of preconditioning samples

16 to a known water content, then exposing the bottom surface of the sample to liquid water

17 and measuring the increase in mass resulting from water absorption. According to the

18 standard conditioning procedure, the samples are conditioned for 18 days. The samples

19 are first placed in a $50{ }^{\circ} \mathrm{C}$ and $80 \%$ relative humidity (RH) environment for three days.

20 Then, the samples are removed from the oven and placed in individually sealed

21 containers where they remain for 15 days, to allow internal moisture to spatially

22 equilibrate before the test begins. 
1 The absorption test involves recording incremental mass change measurements

2 during the first six hours after the sample comes in contact with water and subsequently

3 taking one measurement every day for the next eight days. The amount of absorbed

4 water is normalized by the cross-section area of the specimen exposed to the fluid using

5 Equation 3.1:

$6 \quad I=\frac{m_{t}}{(a \cdot d)}$

7 where: $\mathrm{I}\left(\mathrm{mm}^{3} / \mathrm{mm}^{2}\right)$ is the normalized absorbed water, $\mathrm{m}_{\mathrm{t}}(\mathrm{g})$ is the change in specimen

8 mass at time $\mathrm{t}$; a $\left(\mathrm{mm}^{2}\right)$ is the area of the specimen exposed to water (i.e., that of the

9 bottom face), and $\mathrm{d}\left(\mathrm{g} / \mathrm{mm}^{3}\right)$ is the density of the water (taken to be $0.001 \mathrm{~g} / \mathrm{mm}^{3}$ at 23

$10{ }^{\circ} \mathrm{C}$ ), and the units provided in the ASTM C1585 standard (ASTM 2004) are employed.

11 These absorption measurements are then plotted as a function of the square root

12 of time. The initial sorptivity is determined as the slope of the curve during the first six

13 hours, while secondary sorptivity is determined using the slope of the same

14 measurements between one and eight days, as outlined in ASTM C1585 (ASTM 2004).

15 The initial and secondary sorptivities can be used to evaluate the connectivity of

16 the pore network. Additionally, the secondary sorptivity, combined with exposure

17 conditions, has been used for performing service life predictions (Bentz 2001). 


\section{$1 \quad$ 3.3.3.2 Sample Preparation for Water Absorption Test}

2 Concrete samples were collected using cores, cylinder molds and beams from

3 field mixtures. $100 \mathrm{~mm}$ diameter cylinders samples were collected when cores and

4 cylinder molds were used. When samples were collected on beams, $100 \mathrm{~mm}$ cores were

5 taken from the samples.

6 After cylindrical samples were obtained, $50 \mathrm{~mm} \pm 2 \mathrm{~mm}$ thick samples were cut

7 from the central portion of cylinders with a wet saw. Nine $100 \mathrm{~mm}$ diameter and $50 \mathrm{~mm}$

8 thick samples were prepared for each concrete mixture.

9 After cutting, concrete and mortar samples were conditioned by placing them in

10 environmental chambers at $23 \pm 0.5{ }^{\circ} \mathrm{C}$. Specimens were placed in environmental

11 chambers at three different relative humidities $(50 \pm 1 \%, 65 \pm 1 \%$ and $80 \pm 1 \%)$ until

12 they reached mass equilibrium, which happened after 10 months.

\section{$13 \quad$ 3.3.3.3 Water Absorption Results}

14 Concrete samples were tested during 8 days according with ASTM C 1585.

15 Results are shown on Figure 3.4 to 3.9. 

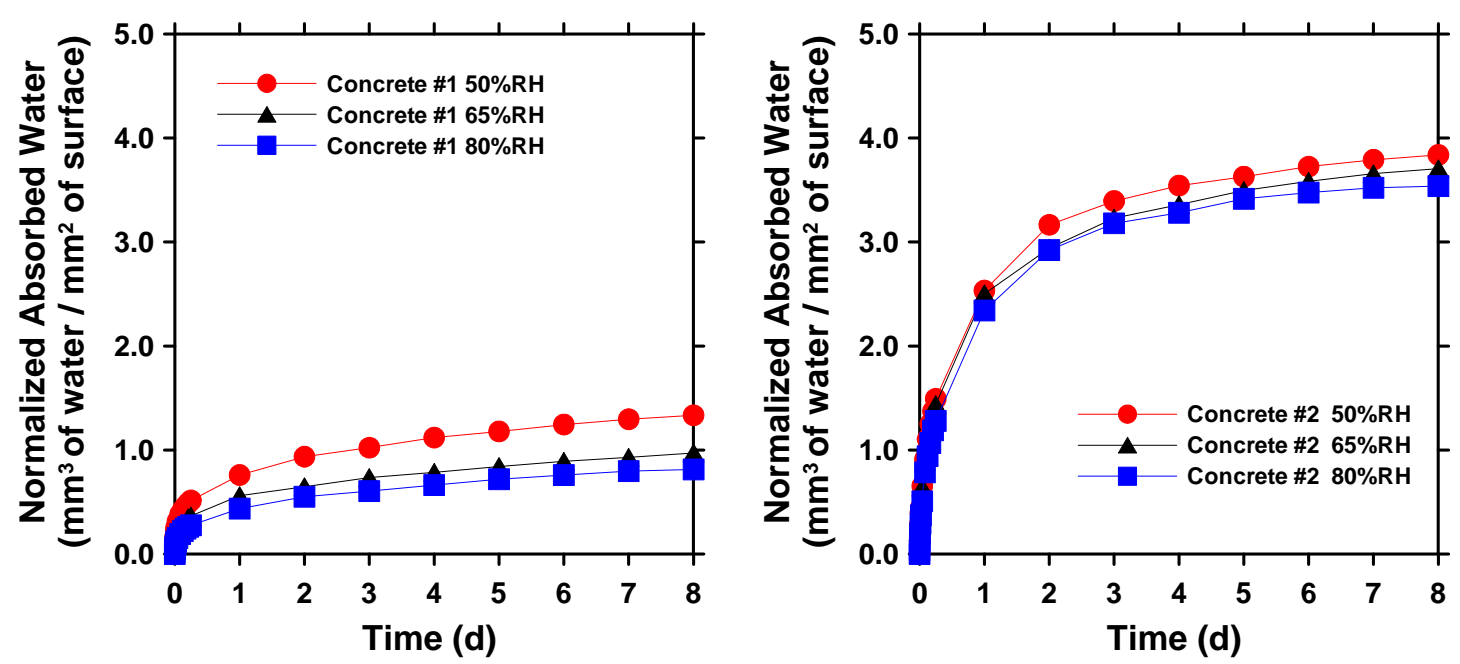

Figure 3.4: Water Absorption Test, Concretes \#1 and \#2: Plain Cement with Standard Aggregate
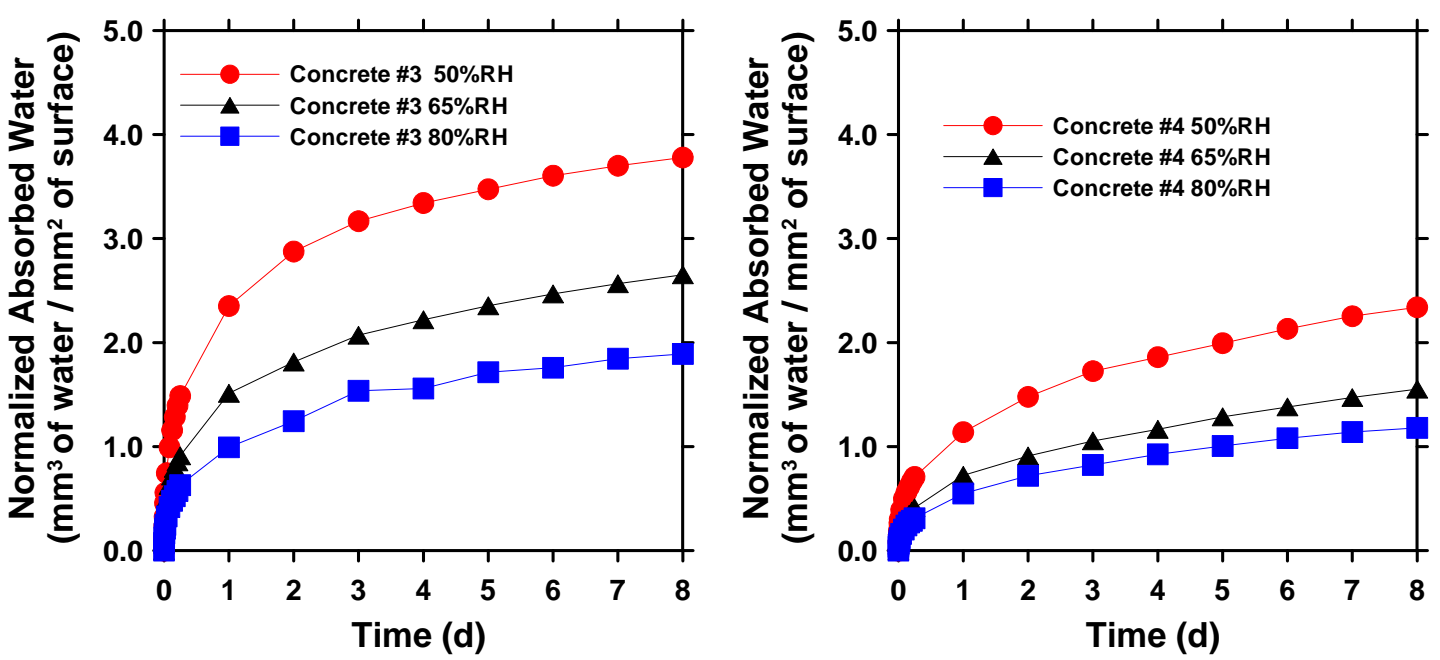

5

Figure 3.5: Water Absorption Test, Concretes \#3 and \#4: Plain Cement with Slag Aggregate 

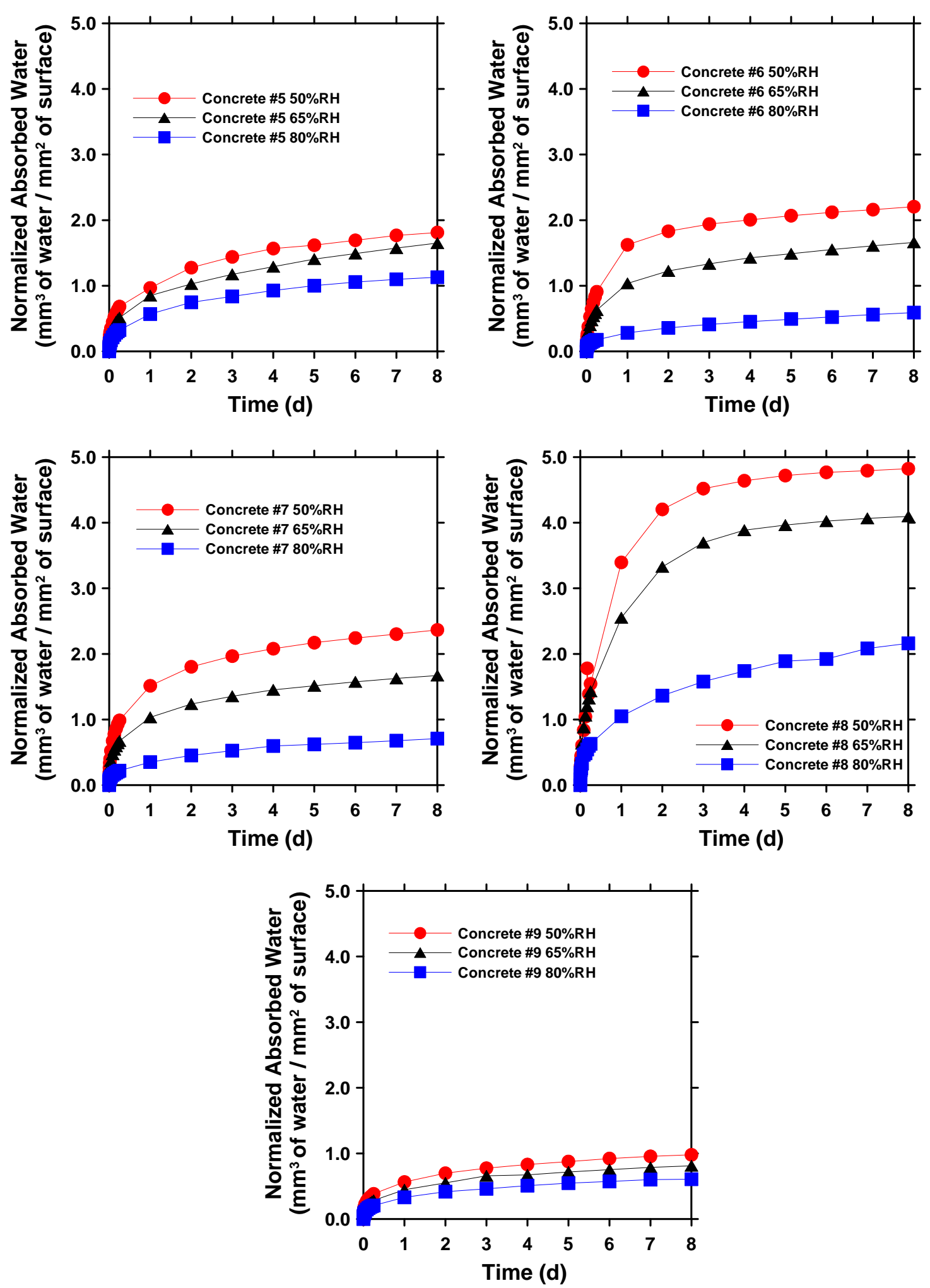

Figure 3.6: Water Absorption Test, Concretes \#5 to \#9: Cement plus Fly Ash with 

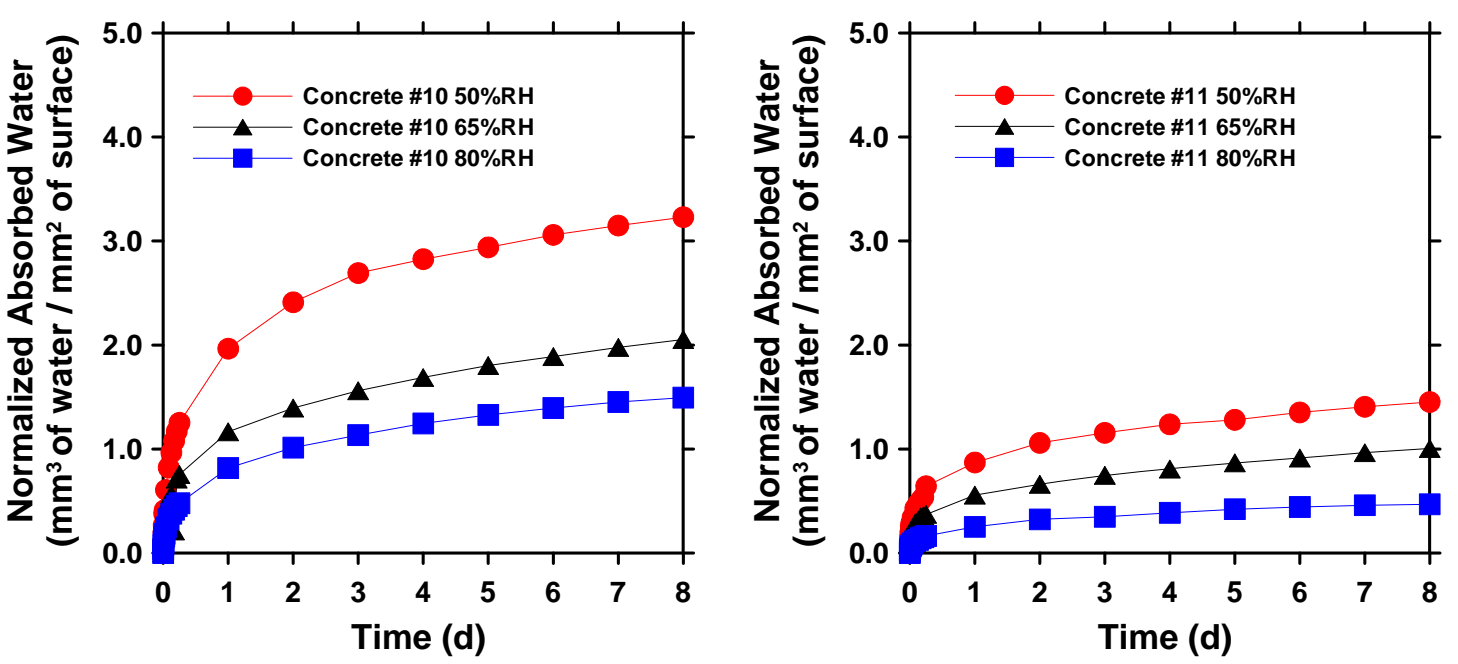

Figure 3.7: Water Absorption Test, Concretes \#10 and \#11 Cement plus Fly Ash with Slag Aggregate
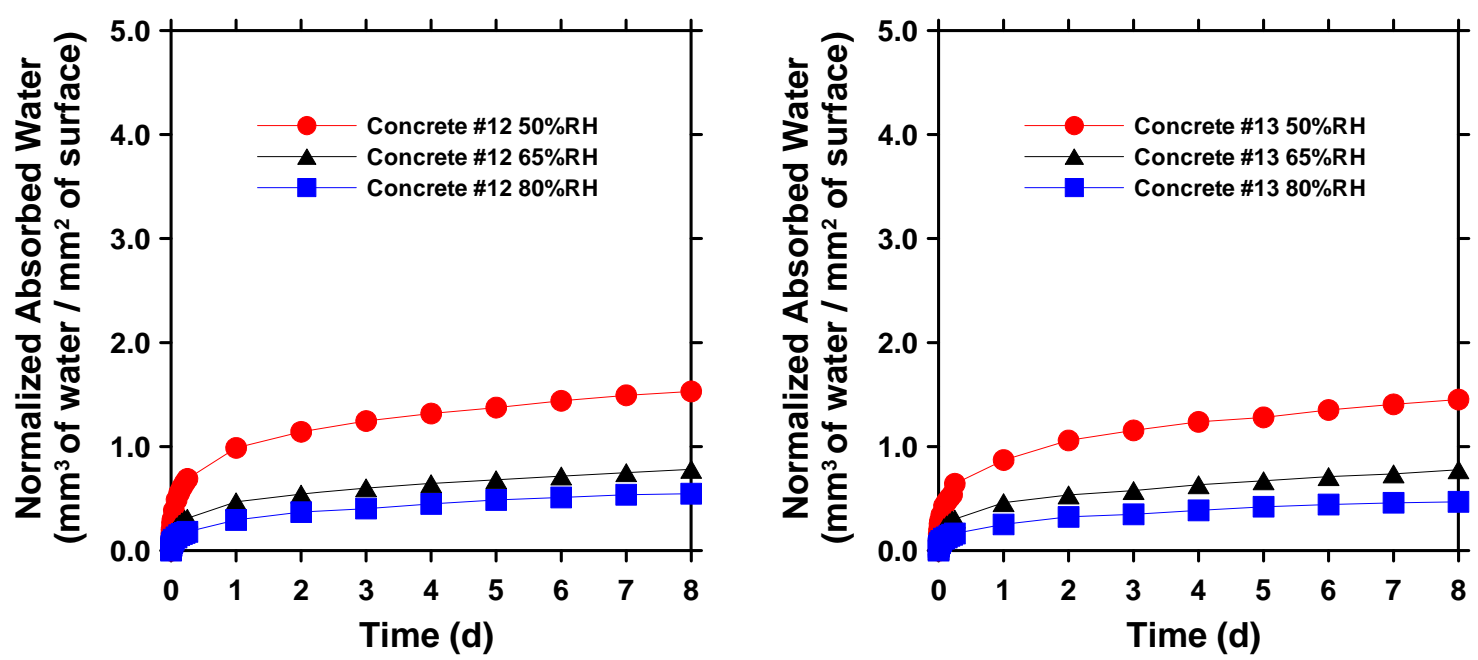

$5 \quad$ Figure 3.8: Water Absorption Test, Concretes \#12 and \#13 Cement plus Fly Ash and 6 Silica Fume with Standard Aggregate 


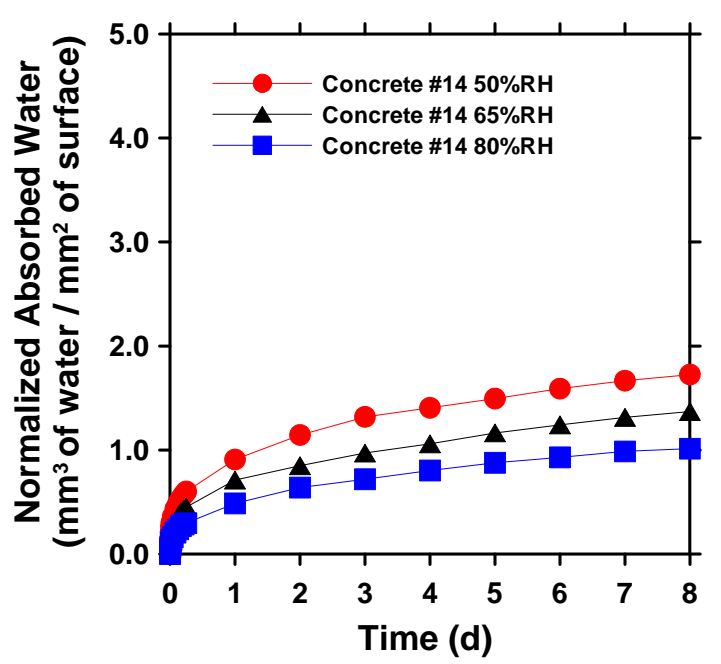

Figure 3.9: Water Absorption Test, Concretes \#14, Cement plus Slag with Slag Aggregate

$4 \quad$ 3.3.3.4 Analysis of the Results

5 The effect of relative humidity on the test results is visible on all tested concretes.

6 The same material conditioned at different relative humidity show different behaviors.

7 The higher the relative humidity (moisture content) the lower the absorption. This is clear

8 example of the importance of the sample preparation for further analysis.

9 Concrete samples containing supplementary materials show in general a lower

10 absorption compared with similar systems containing plain cement.

11 Samples containing slag aggregate tends to absorb more water than concrete

12 samples containing just normal aggregate. This may be explained by the high porosity of

13 the slag aggregate. However, this higher absorption is not an indicator by itself about the

14 overall quality of the concrete.

15 Concrete \#1 and \#2 have similar mixture proportions and are similar age.

16 However after visual inspection, concrete \#2 presents a considerable amount of pores, 
1 evidence of lack of consolidation. This higher porosity of the paste generates a very high

2 absorption value when it is compared with concrete \#1. This is clear evidence of the

3 importance of a good consolidation.

4 Concrete \#3 and \#4 have similar amount of paste and aggregates and both contain

5 slag aggregate in similar proportion, but having a different $w / c$. Concrete $\# 3$ has a $w / c=$

60.43 and $\# 4$ has a $w / c=0.39$. The result shows clearly the effect of a higher w/c on the

7 water absorption at any of the different relative humidities.

8 Concretes \#5 to \#9 represent a widely used mixture proportion for the State of

9 Indiana constituted of binder composed of cement and fly ash. The difference in $w / c$ (in

10 the range of 0.38 to 0.42$)$, the range of cement paste volume (29\% to $34 \%)$, and the

11 different level of fly ash replacement make difficult to compare these results to other

12 mixtures. However, in general results are very similar and comparable with water

13 absorption of concrete containing other supplementary material analyzed in this research.

14 A special note is needed for Concrete \# 8. Visual analysis of this samples shows a

15 very porous structure, what be attributed mainly to a lack of consolidation. This concrete

16 was tested knowing this condition, and the very high water absorption result is consistent

17 with the observed high porosity.

18 Concretes \#10 and \#11 contain similar amounts of cement and fly ash, but

19 concrete \#10 has a higher w/c (0.42 vs. 0.38$)$, a higher amount of cement paste (34.0\%

20 vs. $31.2 \%$, and it is younger than concrete \#11 (2.5 months vs. 6 years). As result,

21 concrete \#10 presents a higher water absorption then concrete \#11.

22 Concretes \#12 and \#13 correspond to two different project having the same

23 mixture proportion: $w / c=0.39,445 \mathrm{lb} / \mathrm{ft}^{3}$ of cement, $125 \mathrm{lb} / \mathrm{ft}^{3}$ of fly ash and $32 \mathrm{lb} / \mathrm{ft}^{3}$ of 
1 silica fume, and a paste volume of $32.9 \%$, and they were pavement with one day of

2 difference. Water absorption test results show very similar results between the projects.

3 This shows a high repeatability of the test under similar conditions.

4 Concrete \# 14 containing slag cement and slag aggregate presents a water 5 absorption comparable with the ones containing fly ash and slag aggregate.

9 relative humidity ( $\mathrm{RH})$ and temperature). The diffusivity is calculated for a cement paste

10 sample with $w / c$ of 0.5 . The same method can be used to obtain the diffusivity of other

11 cement based materials.

\section{$12 \quad$ 3.3.4.1 Desorption Measurements on Cement Paste}

13 The desorption response was measured for a cement paste sample with $5 \mathrm{~mm}$

14 diameter and $1 \mathrm{~mm}$ thickness. The sample was kept seal for 24 hours after casting. After

15 demolding the sample was immediately placed in a tared $180 \mathrm{~mL}$ quartz pan. The pan

16 containing the sample was then suspended from the balance ( $\pm 0.001 \mathrm{mg}$ accuracy) and

17 placed in the relative humidity chamber to equilibrate at $23.0 \pm 0.1{ }^{\circ} \mathrm{C}$ and $97.5 \pm 0.1 \%$

$18 \mathrm{RH}$ for up to $48 \mathrm{~h}$ or until the sample had achieved a stable mass (less than an $0.001 \%$

19 mass change/15 minutes). After the mass of the sample equilibrated (the mass change

20 was less than $0.001 \%$ mass change/15 minutes) the relative humidity was reduced in $5 \%$ 
1 RH steps to $2.5 \% \mathrm{RH}$. The samples were allowed to equilibrate at each humidity. After

2 equilibrating at $2.5 \% \mathrm{RH}$ the samples were dried to $0 \% \mathrm{RH}$.

3 Figure 3.10 illustrates the normalized weight loss of the cement paste sample

4 exposed to the full range of relative humidity (RH). Each step presents the weight loss of

5 the sample at a constant RH which is also plotted on in the graph. All the steps are at

6 constant temperature of $23{ }^{\circ} \mathrm{C}$. The insert in Figure 3.10 is a closer representation of one

7 of the steps.

8

9

10

11 at each step during this experiment the moisture diffusivity can be considered constant

12 since the change in $\mathrm{RH}$ value is small. The change in mass of sample, $\mathrm{M}_{\mathrm{t}}$, as a function of

13 time, t, can be represented using Equation 3.3 (Crank 1980). 
$1 \quad \frac{M_{4}}{M_{n}}=\frac{4}{\pi}\left(\frac{2 t}{\omega^{2}}\right)^{2 / 2}$

2 where $M_{\infty}$ is the mass change at equilibrium, $D$ is is the diffusivity and $L$ is the thickness

3 of the sample. Equation (3.3) can be used to obtain the $D$ at each step by calculating the

4 slop of a curve that is obtained by plotting $\frac{\mathscr{W}_{6}}{W_{\mathrm{s}}}$ verses square root of time.

5 Figure 3.11 illustrates the calculated diffusivity of the cement using Equation

6 (3.3). Diffusivity increases rapidly with RH. The obtained values for diffusivity are in

7 agreement with the reported values in literature (Bazant and Najjar 1972, Bazant 1986,

$8 \quad$ Anderberg and Wadso 2008).

9

10

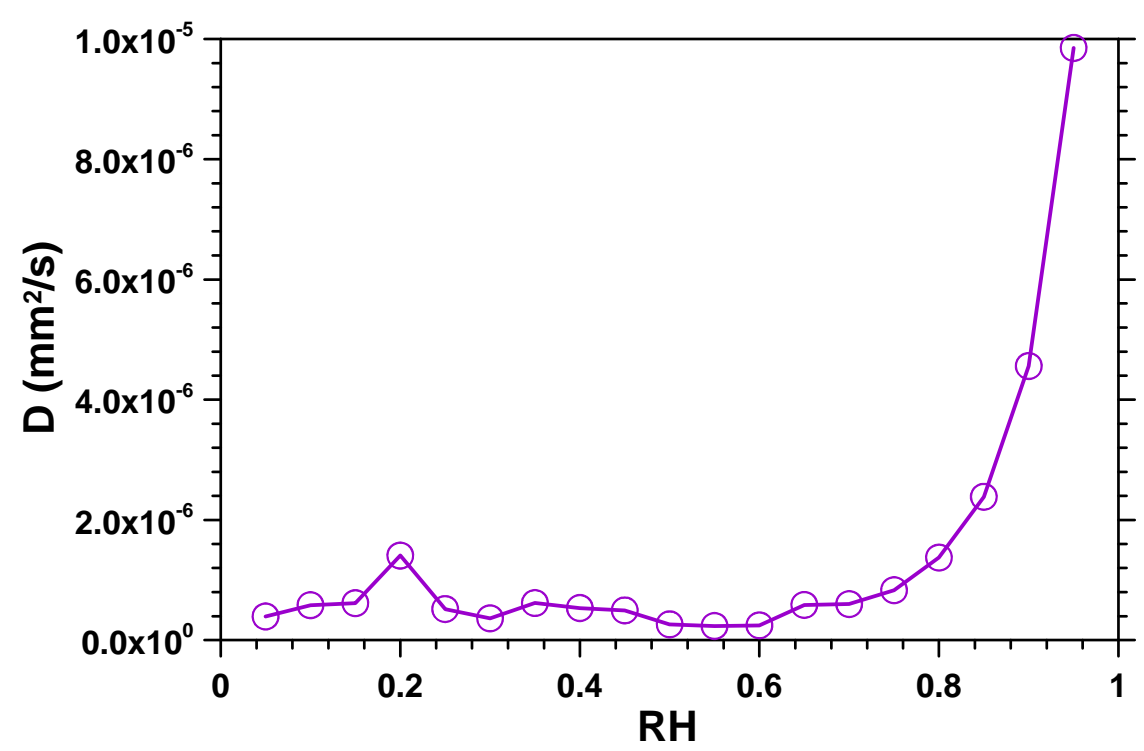

Figure 3.11: Diffusivity of Cement Paste Sample as a Function of RH 
9 day of curing, the samples were demolded and then sealed in double plastic bags for

10 sealed curing. Bags were stored in a room at $23 \pm 1{ }^{\circ} \mathrm{C}$ until samples reached an age of 28

$11 \mathrm{~d}$. After that, cylinders were removed from bags and eighteen $10 \mathrm{~mm} \pm 2 \mathrm{~mm}$ thick

12 samples were cut from the central portion of each cylinder with a wet saw. After cutting,

13 mortar samples were placed in environmental chambers at $23 \pm 0.5{ }^{\circ} \mathrm{C}$ and $50 \pm 1 \%$ for

1418 months.

15 Samples were then vacuum saturated for $24 \mathrm{~h}$. After that, specimens were placed

16 in environmental chambers at six different relative humidities $(93 \pm 1 \%, 87 \pm 1 \%, 75 \pm$

$171 \%, 65 \pm 1 \%$ and $50 \pm 1 \%$ ). Weight was monitored at regular intervals until it reach

18 equilibrium. At the end, all samples were oven dried to express water absorption in term

19 of the dry mass the sample. Figure 3.12 shows the desorption behavior of these mortar 20 samples. 


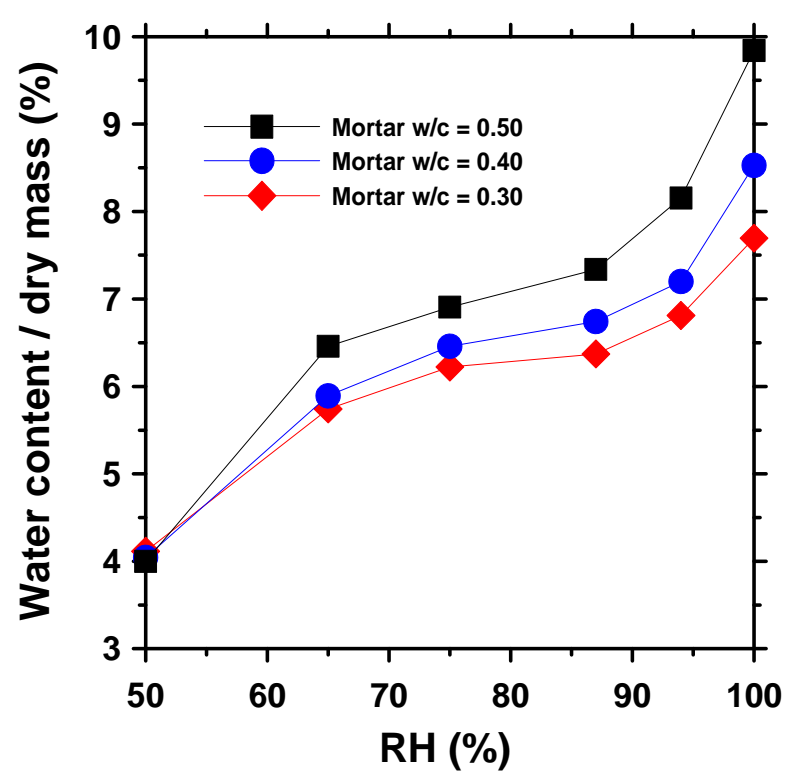

Figure 3.12: Desorption Curve on 18 Months Old Mortar Samples

4 This section has described the absorption behavior of concretes conditioned at

5 different relative humidities. A summary of the general conclusions from the data 6 presented in this chapter are:

- A series of concrete paving mixtures were tested to provide a range of values that were typical for the state of Indiana.

- While similar mixture proportions were used for the mixtures in Indiana differences in the magnitude of water absorbed occurred.

- The standard absorption test ASTM C-1585 is considerably affected by the relative humidity of the samples before starting the test. Comparing samples 
conditioned at different relative humidities can lead to a misunderstanding of the actual absorption behavior. 


\section{$\underline{4.1 \text { Overview }}$}

ASTM C1585 is commonly used to determine the absorption and rate of

5 absorption of water in unsaturated hydraulic cement concretes. ASTM C1585

6 preconditions the samples for a total of 18 days. Unfortunately however, the range of

7 relative humidities that can exist in the samples after this relatively short conditioning

8 period may provide a wide enough variation to considerably influence the results of the

9 test. Three main variables were studied in this program to assess the effect of

10 preconditioning. First, the role of water to cement ratio was investigated by testing

11 mortar samples with $55 \%$ aggregate by volume with four different water-to-cement

$12 \operatorname{ratios}(w / c$ of $0.35,0.40,0.45$ and 0.50$)$. Second, the role of paste volume was

13 investigated by considering samples with $55 \%, 45 \%$, and $35 \%$ aggregate by volume

14 with a $w / c=0.50$. Finally, the effect of conditioning was assessed by exposing all the

15 samples in three different relative humidities $(50 \%, 65 \%$ and $80 \%)$ until they reached

16 mass equilibrium (defined as constant mass over 15 days), taking approximately 14

17 months. Oven dry samples were also prepared and tested for comparison. The results

18 confirm that water absorption testing is considerably influenced by sample preparation.

19 Samples conditioned at $50 \%$ relative humidity can show up to six times greater total 
1 absorption than similar samples conditioned at $80 \%$ relative humidity. Samples that

2 were conditioned in the oven at $105{ }^{\circ} \mathrm{C}$ do not appear to follow a similar trend when

3 compared with specimens conditioned in chambers for the longer duration. The

4 absorption is also influenced by the volume of paste in the samples. The experiments

5 show that a lack of control on moisture content or lack of consideration of the material

6 composition can lead to a misunderstanding of the actual absorption behavior.

11 main mechanisms can be used to describe transport in cementitious systems:

12 permeability, diffusion and absorption. Permeability is the measure of the flow of water

13 under a pressure gradient, while diffusion is the movement of ions due to a concentration

14 gradient. Absorption can be described as the materials ability to take in water by means

15 of capillary suction. All three mechanisms are heavily influenced by the volume of pores

16 as well as the connectivity of the pore network. A large fraction of concrete in service is

17 only partly saturated and the initial ingress of water and dissolved salts is influenced, at

18 least in part, by capillary absorption (Hearn et al., 1994). As such, water absorption has

19 been used as an important factor for quantifying the durability of cementitious systems

20 (Parrot 1992; Hooton et al. 1993; Hearn et al. 1994; Fagerlund 1996; Bentz et al. 2001;

21 Yang 2004; Henkensiefken et al. 2009). Water absorption is the primary focus of this

22 study since it is being increasingly used by specifiers and in forensic studies to provide a 
1 parameter that can describe an aspect of concrete durability. It is also important that these

2 properties be adequately described for use in service life models (Fagerlund 1996; Bentz 3 2001).

\subsubsection{Water Absorption Test}

5 ASTM C1585 (2004) is commonly used to determine the absorption and rate of

6 absorption (commonly referred to as sorptivity) of water in unsaturated hydraulic cement

7 concretes. This test method, based on work reviewed by Hall (1989), consists of

8 preconditioning samples to a known moisture content, then exposing the bottom surface

9 of the sample to liquid water and measuring the increase in mass resulting from water

10 absorption. According to the standard conditioning procedure, samples are conditioned

11 for 18 days. This conditioning period begins by first placing the sample in a $50^{\circ} \mathrm{C}$ and 80

$12 \%$ relative humidity $(\mathrm{RH})$ environment for three days. The samples are then removed

13 from this environment and placed in individually sealed containers where they remain for

1415 days at $23{ }^{\circ} \mathrm{C}$, to allow internal moisture to redistribute throughout the specimens

15 before the test begins.

16 The absorption test involves recording incremental mass change measurements at

17 relatively frequent intervals during the first six hours after the sample comes in contact

18 with water and subsequently taking one measurement every day for the next eight days.

19 The amount of absorbed water is normalized by the cross-section area of the specimen

20 exposed to the fluid using Equation 4.1: 
$1 \quad \mathrm{i}=\frac{\mathrm{m}_{\mathrm{t}}}{(\mathrm{a} \cdot \rho)}$

2 where: $\mathrm{i}\left(\mathrm{mm}^{3} / \mathrm{mm}^{2}\right)$ is the normalized absorbed fluid, $\mathrm{m}_{\mathrm{t}}(\mathrm{g})$ is the change in specimen

3 mass at time $\mathrm{t}$; $\mathrm{a}\left(\mathrm{mm}^{2}\right)$ is the area of the specimen exposed to the fluid (i.e., that of the

4 bottom face), and $\rho\left(\mathrm{g} / \mathrm{mm}^{3}\right)$ is the density of the absorbed fluid (taken to be $1000 \mathrm{~kg} / \mathrm{m}^{3}$ 5 at $\left.23^{\circ} \mathrm{C}\right)$.

6 These absorption measurements are then plotted as a function of the square root of

7 time. The initial sorptivity is determined as the slope of the curve during the first six 8 hours, while secondary sorptivity is determined using the slope of the same

9 measurements between one and eight days, as outlined in ASTM C1585 (2004). It should

10 be noted that these times work well for water though they may not work as well for other

11 fluids with different surface tension and/or viscosity (Spragg et al. 2010).

12 The initial and secondary sorptivities can be used to evaluate the connectivity of

13 the pore network. Additionally, the secondary sorptivity, combined with exposure

14 conditions, has been used for performing service life predictions (Bentz et al. 2001).

16 Water ingress in unsaturated concrete is dominated by capillary suction upon 17 initial contact with water (Martys and Ferraris 1997, Martys 1999, Lockington et al. 18 1999; Hall 2007, Yang et al. 2007). Capillary absorption can be related to the volume of 19 the pores as well as the size (i.e. radius) of the partially empty capillary pores (Figure 
1 4.1). The relation between the equilibrated relative humidity and the radius of the 2 smallest empty pore is given by the Kelvin-Laplace equation (Equation 4.2).

3

$$
\operatorname{Ln}(R H)=\frac{2 \sigma \mathrm{V}_{\mathrm{m}}}{r_{m} R T}
$$

4 where: $R H$ is the relative humidity, $\sigma$ is the surface tension of water (pore solution),

$5 \quad[\mathrm{~N} / \mathrm{m}], V_{m}$ is the molar volume of water, $\left[\mathrm{m}^{3} / \mathrm{mol}\right], r_{m}$ is the average radius of curvature $6[\mathrm{~m}], R$ is the general gas constant, and $T$ is the absolute temperature, $\left[{ }^{\circ} \mathrm{K}\right]$.
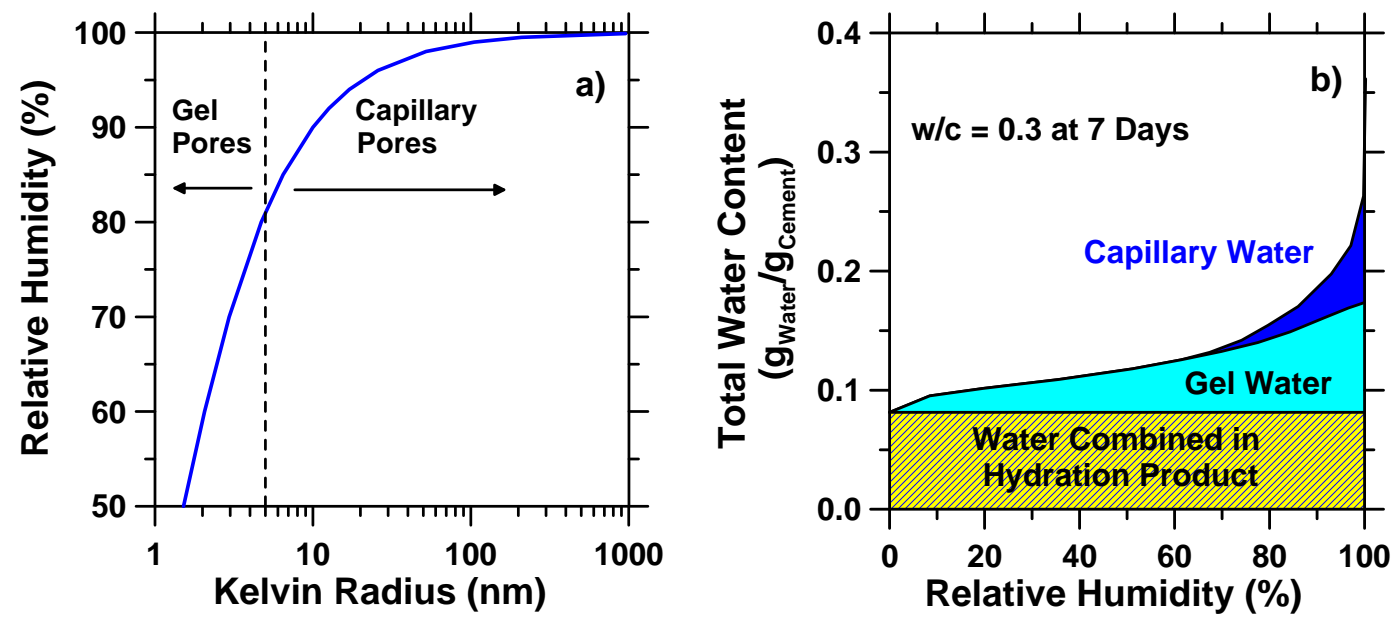

7 Figure 4.1: Relation Between Relative Humidity and Partially Empty Pores in Cement 8 Paste

9 It should be noted that this expression is simplified as it does not consider the 10 effect of water that is absorbed on the walls of the pores. Largely the concrete community 11 has considered two sizes of pores as introduced by Powers (1946). The gel pores are 
1 considered to be small pores $(<10 \mathrm{~nm}$ diameter) that are a part of the hydration products.

2 Capillary pores are larger pores that occur due to excess water. Capillary porosity is

3 particularly of concern in transport, as is the interconnectivity of the capillary pores.

4 Figure 4.1 shows a conceptual illustration after Powers (1946) which used a

5 desorption isotherm to illustrate the volume of water located in the different size pores at

6 different relative humidities.

7 The relative humidity used to condition the sample prior to the sorption test can

8 have a significant impact on the results (ASTM 2004). Previous test results by Parrot

9 (1991, 1994) indicated that the water absorption rate was very sensitive to the moisture

10 content of the concrete, particularly at relative humidities above $60 \%$ which were

11 common for field exposure. Water leaves the largest accessible pores first. It can be seen

12 from Figure 4.1 that capillary pores occupy the range of humidity from approximately 80

$13 \%$ to $100 \% \mathrm{RH}$. As such, initially upon drying water leaves the capillary pores. The

14 lower the relative humidity, the greater the total volume of pores that are empty and

15 available to be filled with water during the sorption test. Further, the lower humidity will

16 empty smaller pores and create a higher suction force in the materials resulting in a

17 greater sorption rate and a larger overall total absorption.

18 According to ASTM C1585, the standardized test conditioning will generally

19 provide an internal relative humidity similar to relative humidities found near the surface

20 in some field concrete structures (DeSouza et al. 1997, DeSouza et al. 1998, ASTM

21 2004). This range of relative humidities can represent what is found in samples in the

22 field; however, it is wide enough to affect considerably the results of the test. 
1 Results presented in Chapter 8 shows that the relative humidity of samples that

2 were kept in the field under different exposure conditions was in the range of $80 \%$ to 100

$3 \%$ depending on the type of exposure, which is somewhat higher than what is mentioned

4 in ASTM C-1585.

7 influence of conditioning relative humidity (oven dry, $50 \%, 65 \%$ and $80 \% \mathrm{RH}$ ) on the

8 results of sorption tests performed on mortars with different $w / c$, containing a fixed

9 volume of aggregate. Second, this research will examine the influence of the volume of

10 aggregate (or equivalently the paste content) on the results of sorption testing. Third, this

11 research will examine the effect of the conditioning method proposed in ASTM C15851204.

\subsubsection{Chapter Objectives}

The objectives of this research are threefold. First, this research will examine the

\section{$\underline{4.3 \text { Materials }}$}

An ASTM C150 Type I ordinary portland cement (OPC) was used in this study, with a Blaine fineness of $370 \mathrm{~m}^{2} / \mathrm{kg}$ and an estimated Bogue composition of $56 \% \mathrm{C}_{3} \mathrm{~S}$, $16 \% \mathrm{C}_{2} \mathrm{~S}, 12 \% \mathrm{C}_{3} \mathrm{~A}, 7 \% \mathrm{C}_{4} \mathrm{AF}$ and a $\mathrm{Na}_{2} \mathrm{O}$ equivalent of $0.68 \%$ by mass.

A polycarboxylate-based high-range water-reducing admixture (HRWRA) was added in varying rates as indicated in Table 4.1 depending on the mixture proportions to maintain similar consistencies (i.e., workability). The sand used was natural river sand 
1 with a fineness modulus of 2.71 , an apparent specific gravity of 2.58 , and a water

2 absorption of $1.8 \%$ by mass.

4 Six different mixtures were prepared in total. Four mixtures were mortars with a

5 single volume fraction of fine aggregate (55\% of the total volume) and different $w / C$

$6(0.35,0.40,0.45$, and 0.50$)$. These mixtures were designated as 55/0.35, 55/0.40, 55/0.45

7 and 55/0.50 with the number on the left representing the volume fraction of fine

8 aggregate and the number on the right representing $w / c$. Additionally, two other mortars

9 were prepared with $w / c$ of 0.50 , but with different volume fractions of fine aggregate (35

$10 \%$ and $45 \%$ of the total volume). They were designated as $35 / 0.50,45 / 0.50$. A list of the

11 mixture proportions can be found in Table 4.1.

12

Table 4.1: Mixture Proportions in Saturated Surface Dry (SSD) Conditions

\begin{tabular}{|c|c|c|c|c|c|c|}
\hline Material & $\mathbf{5 5 / 0 . 3 5}$ & $\mathbf{5 5 / 0 . 4 0}$ & $\mathbf{5 5 / 0 . 4 5}$ & $\mathbf{5 5 / 0 . 5 0}$ & $\mathbf{4 5 / 0 . 5 0}$ & $\mathbf{3 5 / 0 . 5 0}$ \\
\hline Volume fraction of aggregate & $55 \%$ & $55 \%$ & $55 \%$ & $55 \%$ & $45 \%$ & $35 \%$ \\
\hline $\mathbf{w} / \mathbf{c}$ & 0.35 & 0.40 & 0.45 & 0.50 & 0.50 & 0.50 \\
\hline Cement $\mathbf{( k g / \mathbf { m } ^ { 3 } )}$ & 673 & 626 & 585 & 549 & 671 & 793 \\
\hline Water $\mathbf{( k g / \mathbf { m } ^ { 3 } )}$ & 235 & 250 & 263 & 275 & 336 & 397 \\
\hline Fine Aggregate $\mathbf{( k g / \mathbf { m } ^ { 3 } ) , \mathbf { S S D }}$ & 1442 & 1442 & 1442 & 1442 & 1180 & 918 \\
\hline HRWRA (g/ $\mathbf{1 0 0} \mathbf{g}$ cement) & 0.60 & 0.40 & 0.20 & 0.00 & 0.00 & 0.00 \\
\hline
\end{tabular}


1 of water was corrected by the absorption of the aggregate. The water and cement were

2 conditioned for $24 \mathrm{~h}$ at room temperature prior to mixing.

5 curing, the samples were demolded and then sealed in double plastic bags for sealed

6 curing. Bags were stored in a room at $23 \pm 1{ }^{\circ} \mathrm{C}$ until samples reached an age of $28 \mathrm{~d}$.

7 After that, cylinders were removed from bags and three $50 \mathrm{~mm} \pm 2 \mathrm{~mm}$ thick samples

8 were cut from the central portion of each cylinder with a wet saw.

9 After cutting, samples were conditioned by placing them in environmental

10 chambers at $23 \pm 0.5^{\circ} \mathrm{C}$. Specimens from mixtures $55 / 0.35,55 / 0.40,55 / 0.45$ and $55 / 0.50$

11 were placed in environmental chambers at three different relative humidities $(50 \pm 1 \%$,

$1265 \pm 1 \%$ and $80 \pm 1 \%$ ). Specimens from mixtures $35 / 0.50$ and $45 / 0.50$ were placed in an

13 environmental chamber at $50 \pm 1 \%$ relative humidity. Samples were kept in the

14 environmental chamber until they reached mass equilibrium, defined as negligible mass

15 change over a 15 day period. Mixture $55 / 0.35$ placed at $50 \pm 1 \%$ relative humidity

16 required the longest period of time (14 months) to reach mass equilibrium. However, all

17 samples were maintained in the chambers for 14 months to test them all at the same age.

18 Additional specimens from mixtures 55/0.35, 55/0.40, 55/0.45 and 55/0.50 were

19 placed at $50 \pm 1 \% \mathrm{RH}$. After the 14 months, these specimens were dried in an oven at

$20 \quad 105 \pm 2{ }^{\circ} \mathrm{C}$ until they reached mass equilibrium.

21 Once the samples were removed from the chambers or from the oven, the side 22 surface (i.e. outer circumference) was sealed with epoxy and the top surface was covered 
1 with plastic to avoid evaporation from the sample during testing. After the samples were

2 prepared, testing occurred in accordance with ASTM C1585-04 (2004). Specimens from

3 mixtures $55 / 0.35,55 / 0.40,55 / 0.45$ and $55 / 0.50$ were tested over a period of 90 days.

4 Specimens from mixtures $35 / 0.50$ and $45 / 0.50$ were tested over a period of 8 days.

5 Two additional $100 \mathrm{~mm} \times 200 \mathrm{~mm}$ cylinders were cast for each mortar mixture.

6 After one day of curing, the samples were demolded and then sealed in double plastic

7 bags for sealed curing. Bags were stored in a room at $23 \pm 1{ }^{\circ} \mathrm{C}$ until samples reached an

8 age of $28 \mathrm{~d}$. After that, cylinders were removed from bags and $10 \mathrm{~mm} \pm 2 \mathrm{~mm}$ thick

9 samples were cut from the central portion of each cylinder with a wet saw. After cutting,

10 mortar samples were vacuum saturated for $24 \mathrm{~h}$. After that, specimens were placed in

11 environmental chambers at six different relative humidities $(93 \pm 1 \%, 87 \pm 1 \%, 80 \pm 1$

$12 \%, 75 \pm 1 \%, 65 \pm 1 \%$ and $50 \pm 1 \%$ ) to determine their desorption isotherms.

\subsubsection{Desorption Isotherms}

15 Figure 4.2 shows the desorption isotherm curves measured using $10 \mathrm{~mm}$ thick 16 samples. Mass change was monitored at regular intervals until it reached equilibrium,

17 defined as no mass change over a 15 day period. At the end, all samples were oven dried 18 to express water absorption in terms of the dry mass of the sample. 
1 It can be noticed that while the values of the moisture content are similar at $50 \%$

2 and lower RH, as it refers to the small gel pore system (Powers et al. 1946), the capillary

3 pores at high $\mathrm{RH}$ are strongly influenced by the $w / c$.

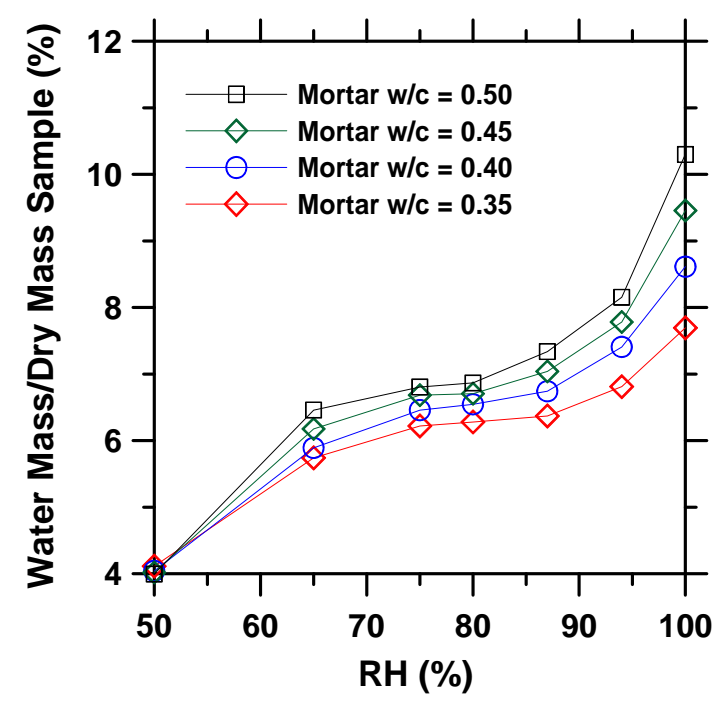

$5 \quad$ Figure 4.2: Desorption Curve on 18 Months Old Mortar Samples (Typical Standard Deviation in the Average of 3 Samples is Lower Than $0.2 \%$ )

\section{$8 \quad$ 4.5.2.1 Effects of Relative Humidity on Sorption Test}

9 Figure 4.3 shows the absorbed water during the 90 days of testing performed on

10 mortars conditioned at different relative humidities (mixtures 55/0.35, 55/0.40, 55/0.45

11 and 55/0.50). It can be noticed that the water absorption is very sensitive to the relative

12 humidity at which the specimens were pre-conditioned before testing. In each case, as the

13 conditioning relative humidity increases, the absorption decreases. 

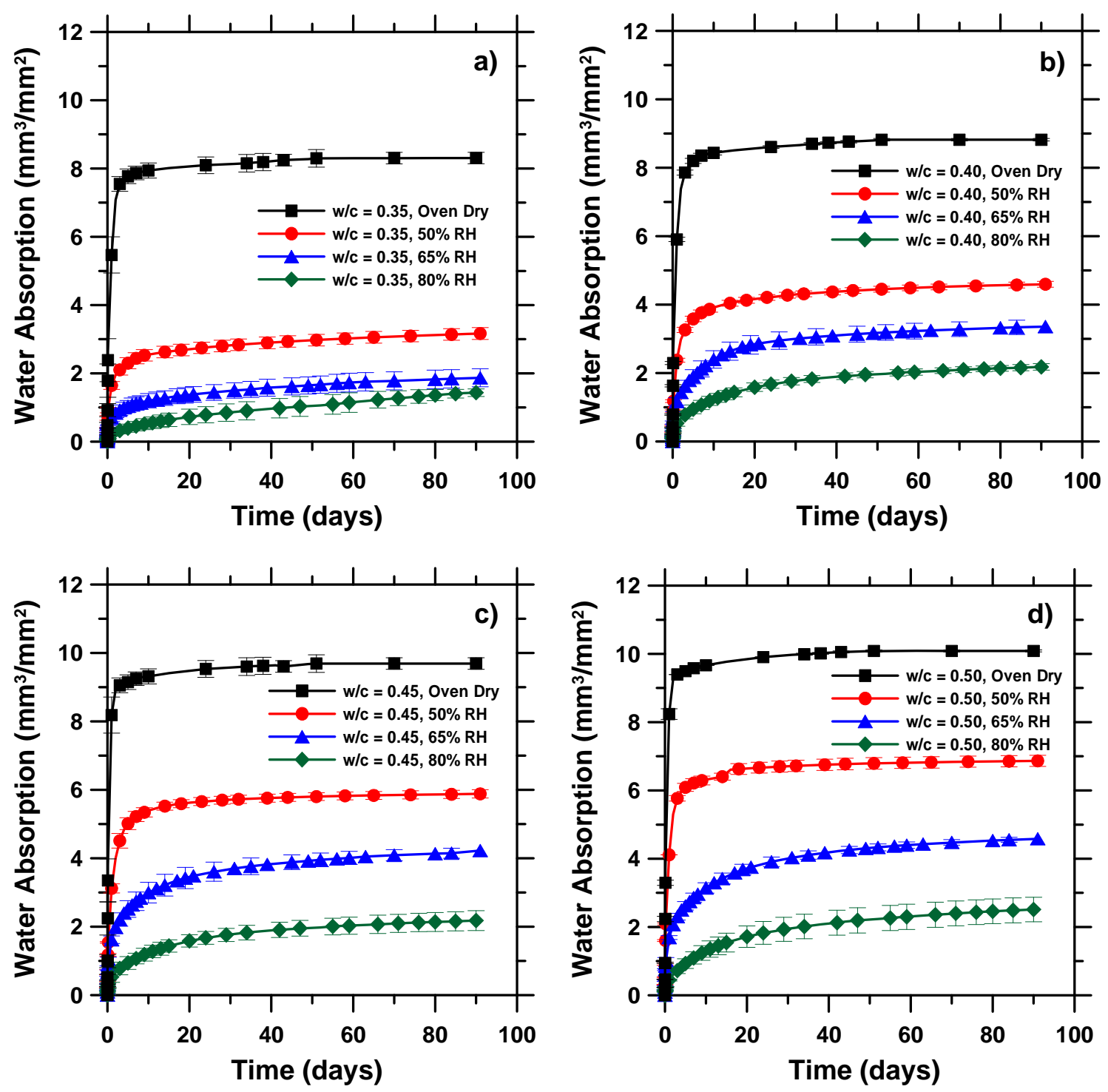

Figure 4.3: Absorbed Water in Mortars as a Function of Relative Humidity: a) Mixture $55 / 0.35$, b) Mixture 55/0.40, c) Mixture 55/0.45, d) Mixture 55/0.50. Error Bars Represent the Standard Deviation for the Average of Three Samples.

These results can be viewed in a slightly different manner if they include the

7 initial amount of water held in the pores before the test. In order to do this, samples were

8 oven dried at the end of the sorption test to calculate the amount of water they held before

9 starting the test. Additional specimens that were kept at $50 \pm 1 \% \mathrm{RH}$ during the 14 
1 months were oven dried and then saturated by the procedure described in ASTM C642-

206 to measure the total amount of interconnected porosity in the systems. Results from

3 Figure 4.3 were then normalized by the total amount of pores in the system, which can be

4 viewed as the degree of saturation of the sample as a function of time. This is presented

5 in Figure 4.4. Figure 4.5 shows the total degree of saturation for the samples after 90 6 days.

Figures 4.4 and 4.5 show that samples prepared at different relative humidities

8 with a low $w / c($ e.g. $w / c=0.35)$ do not reach values near to saturation even after 90 days

9 of being in contact with water. It may be attributed to the refined pore network of this low

$10 \mathrm{w} / \mathrm{c}$ system which makes it difficult for water to move through the sample to fill all the

11 pores. This is commonly referred to as depercolation, which occurs after different

12 hydration times for different $w / c$ (Powers et al. 1959).

13 In contrast after 90 days, samples prepared with a higher $w / c($ e.g. $w / c=0.50)$

14 reach much higher levels of saturation. It can be noted from Figure 4.4 that samples

15 conditioned at $50 \% \mathrm{RH}$ reach values near saturation after about 40 days of testing,

16 similar to what is obtained with oven dry samples. Again this may be attributed to the

17 connectivity of the pore network and the size of these pores. In this case, a more

18 interconnected pore network will facilitate the movement of water to the interior of the

19 specimens and the diffusion of water vapor out of the sample. However, when these

20 samples were conditioned at higher relative humilities (65 and $80 \% \mathrm{RH})$, the amount of

21 initially retained water is high enough to reduce the diffusion of vapor out of the sample.

22 As a result, this may explain why the level of saturation of these specimens is lower. 

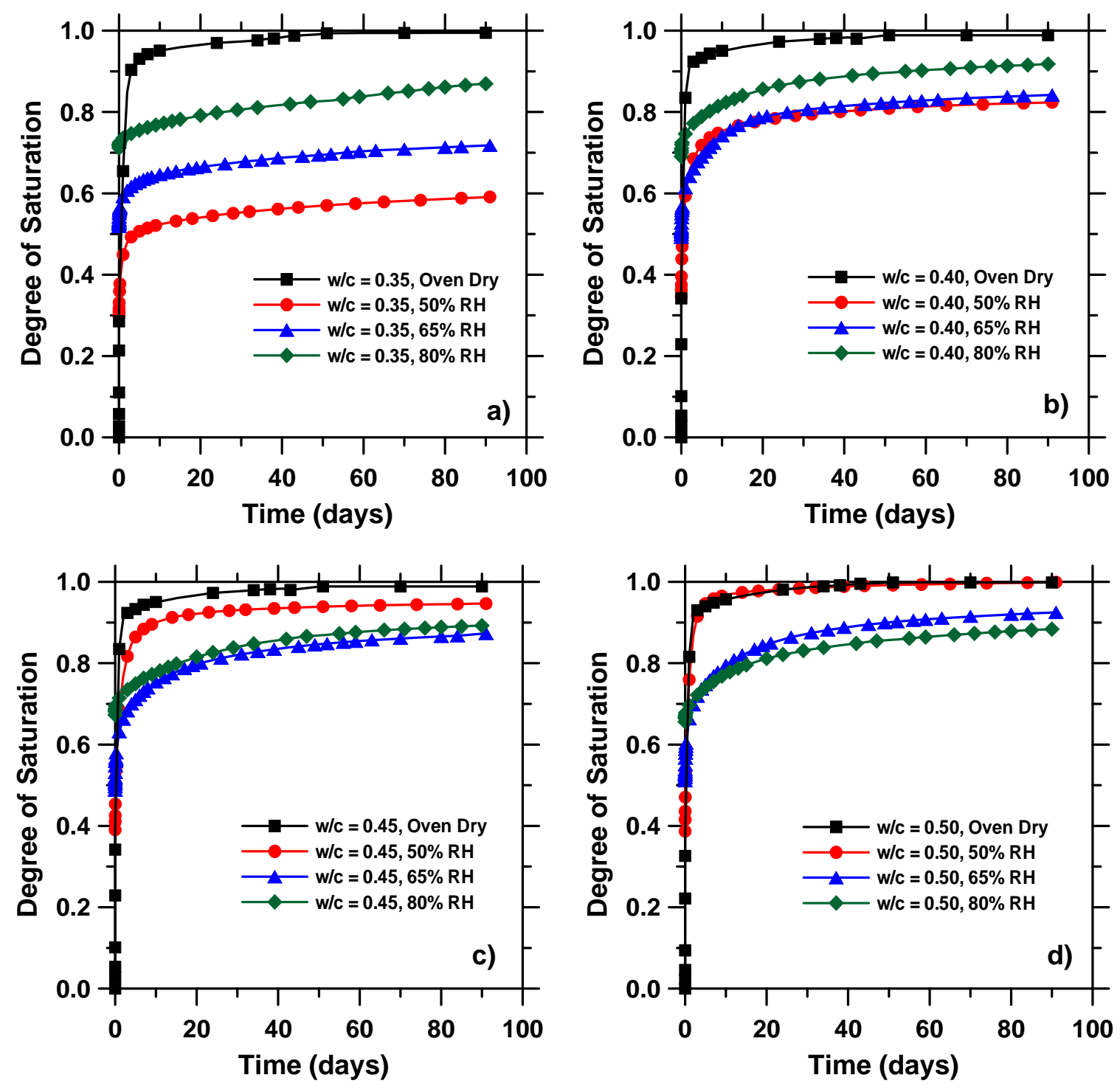

3 Figure 4.4: Degree of Saturation as a Function of Time During the Water Absorption 4 Test: a) Mixture 55/0.35, b) Mixture 55/0.40, c) Mixture 55/0.45, d) Mixture 55/0.50. 5 Typical Standard Deviation of the Average of Three Samples is Lower than 0.02 Points 


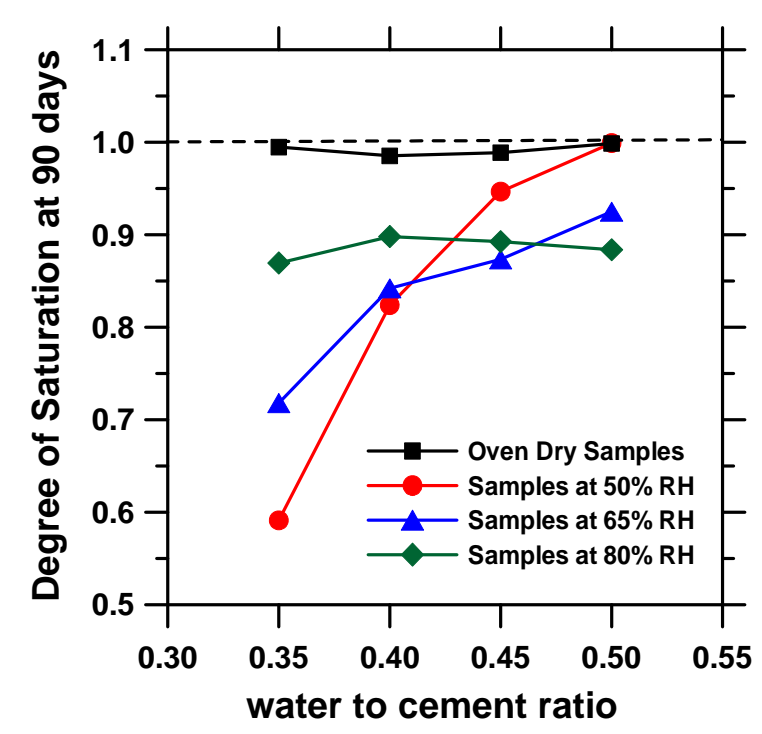

2 Figure 4.5: Degree of Saturation at 90 Days in Contact with Water as a Function of the 3 $w / c$.

$4 \quad$ 4.5.3 Effects of Relative Humidity on the Amount of Absorbed Water after 8 Days

$5 \quad$ Figure 4.6 shows the cumulative water that was absorbed after 8 days of testing

6 performed on mortars conditioned at different relative humidities, expressed as a function

7 of $w / c$ (Figure 4-6a) and as a function of the relative humidity (Figure 4.6b).

$8 \quad$ Figure 4.6 a shows that mixture 55/0.50 can exhibit six times higher absorption

9 when the samples are conditioned at $50 \% \mathrm{RH}$ compared with similar samples 10 conditioned at $80 \% \mathrm{RH}$. 

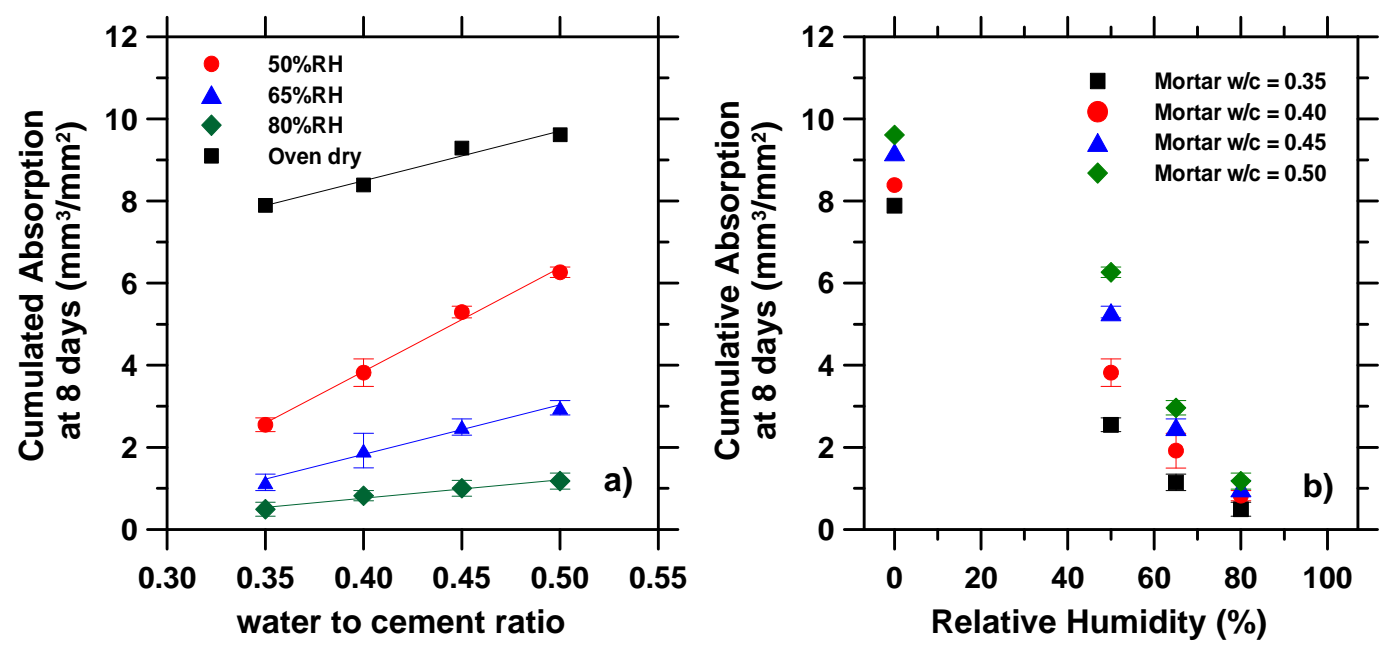

Figure 4.6: Cumulative Absorption at 8 Days for Mortars Versus: a) w/c, b)

3 Relative Humidity. Solid Lines are Provided only to Show a General Tendency in 4 the Data. Error Bars Represent the Standard Deviation on the Average of 3 Samples.
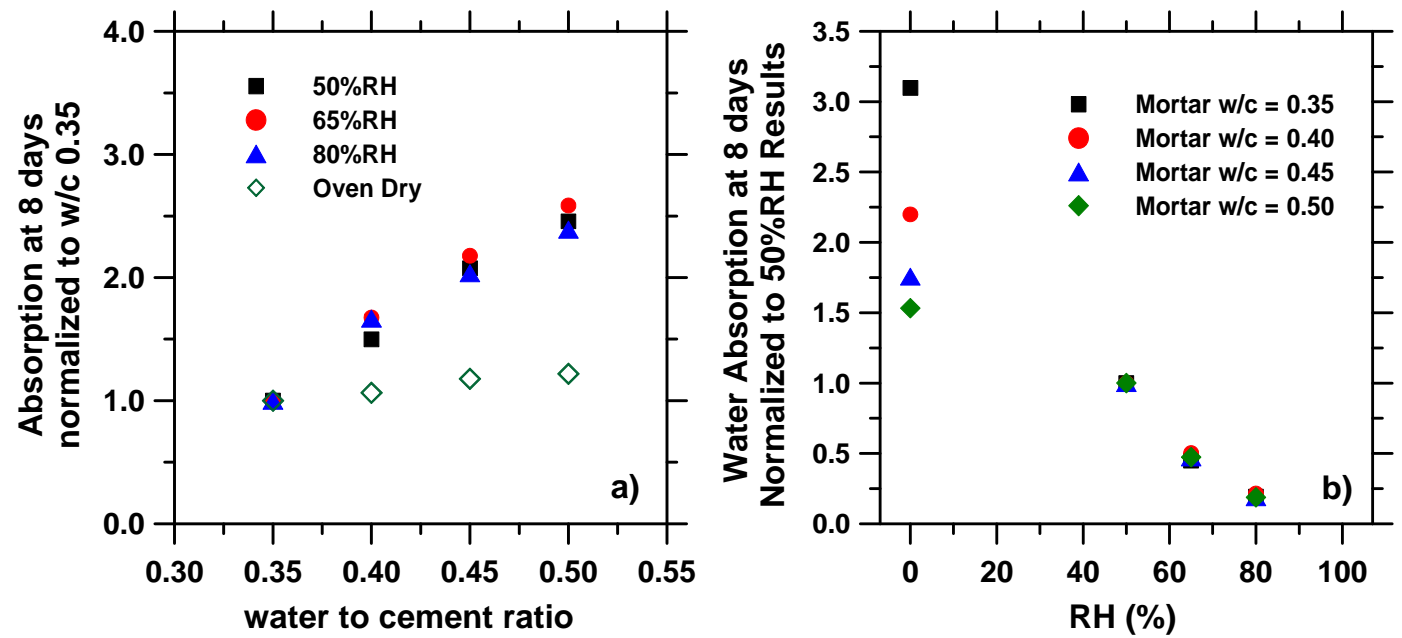

6 Figure 4.7: Cumulative Absorption at 8 Days Versus w/c and Relative Humidity: a)

7 Normalized to Absorption of Mixture 55/0.35, b) Normalized to Absorption at $50 \%$ RH.

Figure 4.7 shows a normalization of the data presented in Figure 4.6. In Figure

$94.7 \mathrm{a}$ the normalization is made with respect to the absorption of samples with $\mathrm{w} / \mathrm{c}=0.35$

10 (mixture 55/0.35). In Figure 4.7b the normalization is made with respect to the absorption 
1 of samples conditioned at $50 \%$ relative humidity. It can be seen that the values follow a

2 consistent trend in each case, except for the oven dry samples.

3

5 the square root of time during the first six hours of test (ASTM 2004).

7 absorption when the samples are conditioned at $50 \% \mathrm{RH}$ compared with similar samples

8 conditioned at $80 \% \mathrm{RH}$.

9 It needs to be noted that the oven dry samples show a much higher initial

10 sorptivity, due to the fact that the gel's capillary pores are empty and possibly to

11 microcracking. While the increase in sorptivity is observed to be linear for the specimens

12 conditioned at $50 \%, 65 \%$ and $80 \%$ relative humidity, this trend appears to break down

13 for the oven dry samples which may be attributed to micro-cracking generated during the

14 sample preparation (Hwang and Young 1984; Chatterji 1976; Bisschop 2002; Samaha

15 and Hoover 1992; Yang et al. 2006). 

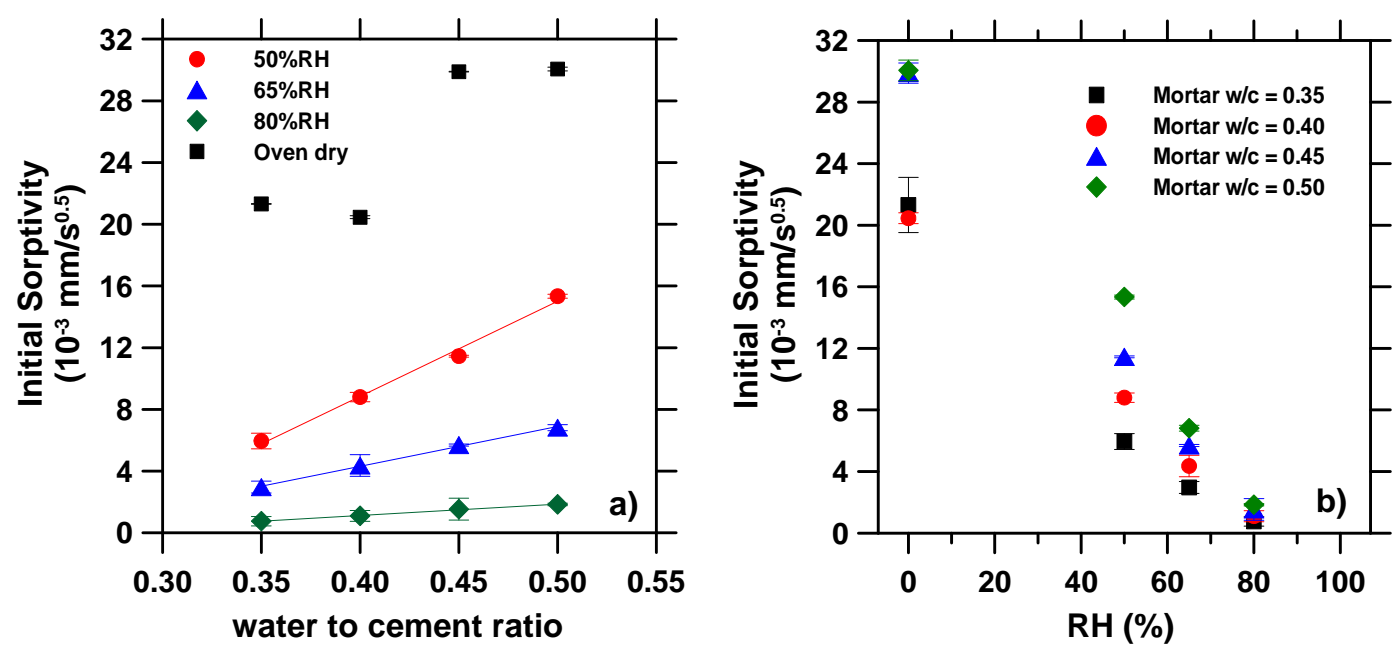

Figure 4.8: Initial Absorption on Mortars Conditioned at Different RH Function of: a) $w / c$, b) Relative Humidity. Solid Lines are Provided to Show a General Tendency in the

Data. Error Bars Represent the Standard Deviation on the Average of 3 Samples.

\subsubsection{Effects of Relative Humidity on Secondary Sorptivity}

Figure 4.9 shows the secondary sorptivity calculated as the slope of the absorption

7 vs. the square root of time between $1 \mathrm{~d}$ and $8 \mathrm{~d}$ of testing. Trends are similar to those

8 observed for the initial sorptivity. However, it needs to be noted that samples that were

9 oven dry prior to the test present a considerably lower secondary absorption with respect

10 to the samples conditioned in environmental chambers. This may be explained by the

11 high initial absorption of the oven dry samples shown in Figure 4-8. During this initial

12 absorption it can be noticed that since a majority of the water was already absorbed in the

13 first hours of the test, the secondary rate of absorption will be much lower. It can also be

14 expected that microcracking enabled a more rapid ingress of water (Yang, 2004). 

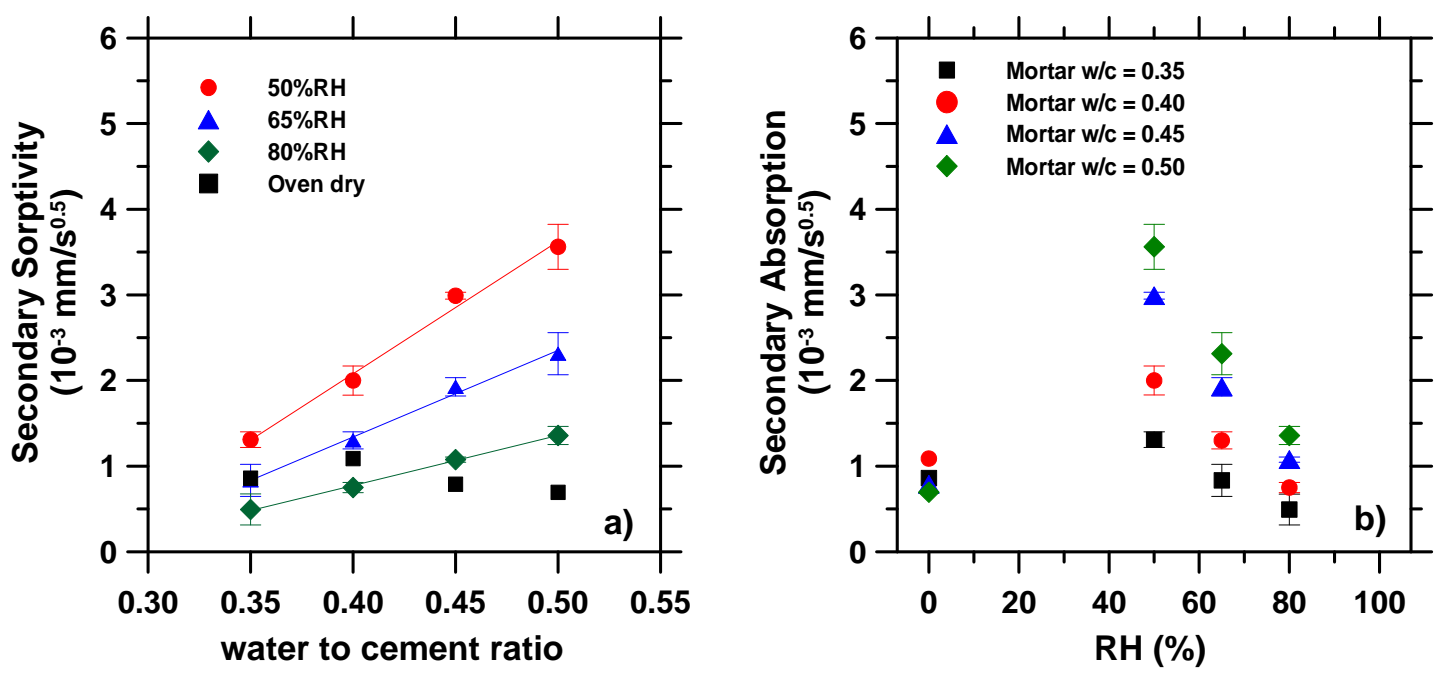

Figure 4.9: Secondary Absorption on Mortars Conditioned at Different RH Function of: a) $w / c$, b) Relative humidity. Solid Lines are Provided to Show a General Tendency in the Data. Error Bars Represent the Standard Deviation on the Average of 3 Samples.

Figure 4.9 shows a similar trend to what was noted in the case of total absorption

6 and initial sorptivity, namely that the secondary sorptivity of samples conditioned in

7 chambers exhibits a consistent trend when the results are plotted against the $\mathrm{w} / \mathrm{c}$ or the

8 relative humidity at which samples were conditioned. However, samples that are

9 conditioned by drying them in an oven at $105^{\circ} \mathrm{C}$ do not follow the same tendency.

10 4.6 Effects of Initial Moisture of Samples on ASTM C1585 Conditioning Method

11 At the age of 24 months, samples from each mixture conditioned at the three

12 different relative humidities were removed from the chambers. The side surface was

13 sealed with epoxy to be then "re-conditioned" using the 18 day procedure described in

14 ASTM C-1585. In addition, three other samples from each mixture were saturated

15 following the procedure described in ASTM C642 (2006), to then be "re-conditioned" 
1 following the same 18 day procedure. After samples were fully prepared, testing was

2 performed in accordance with ASTM C-1585 over a period of 8 days, with results

3 provided in Figure 4.10. In addition, Figure 4.11 shows the calculated initial and

4 secondary sorptivities from these tests. Secondary sorptivity values are not reported when

5 the correlation coefficient is lower than 0.98 .

6 Figure 4.10 and 4.11 show that the 3 days of controlled drying at $50 \pm 2{ }^{\circ} \mathrm{C}$ and 80

$7 \% \mathrm{RH}$ followed by the 15 days for internal moisture equilibration is not capable of

8 eliminating the effects of the "moisture history". These results suggest that the ASTM

9 C1585 preparation method does not prepare all the samples to the same water content

10 before a water absorption test. As such this accelerated method can make a substantial

11 difference in how the data is interpreted. This may be due to moisture hysteresis effect

12 (Nilson 1980). It should be noted that this can be a concern for field samples evaluated

13 using this method, as their as-received relative humidities may easily vary between the

14 extremes examined in this study.

16 Figure 4.12 shows the absorbed water during 8 days of testing performed on

17 mortars containing different volumes of aggregate (mixtures 55/0.50, 45/0.50 and

$1835 / 0.50$ ) conditioned at $50 \%$ relative humidity. In Figure 4.12a the effect of a higher

19 volume of paste is observed as the mixture containing the lower volume of aggregate has

20 the higher absorption. However, when the results are normalized by the volume of paste

21 (volume of the main absorbent material), a reversal in the order of the samples is 
1 observed (Figure 4.12b). The samples with the higher volume of aggregates have a higher 2 absorption.
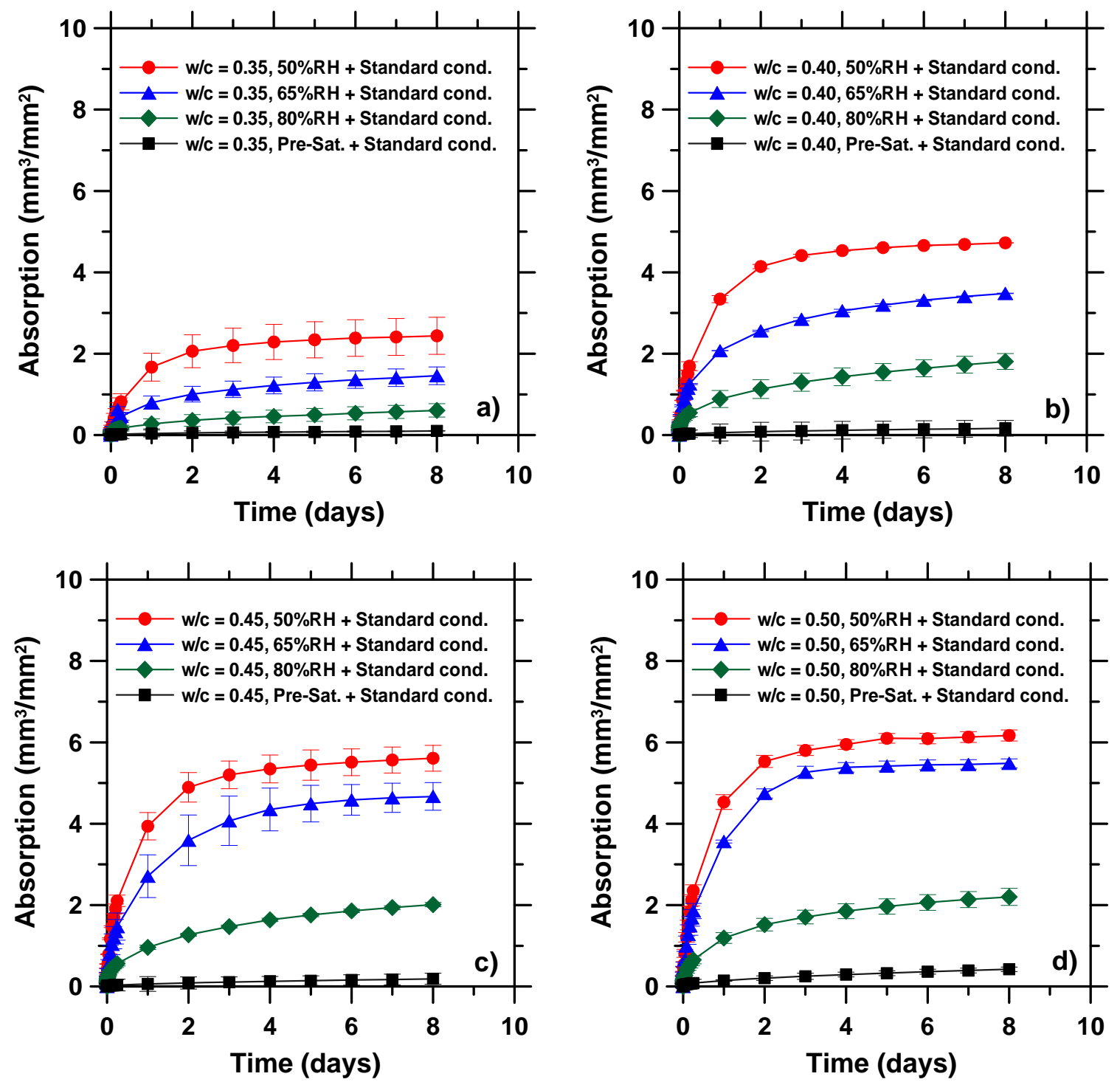

5 Figure 4.10: Effect of Initial Moisture on the Conditioned Procedure Established in 6 ASTM C1585-04: a) Mixture 55/0.35, b) Mixture 55/0.40, c) Mixture 55/0.45, d) Mixture $755 / 0.50$. Error Bars Represent the Standard Deviation for the Average of Three Samples. 

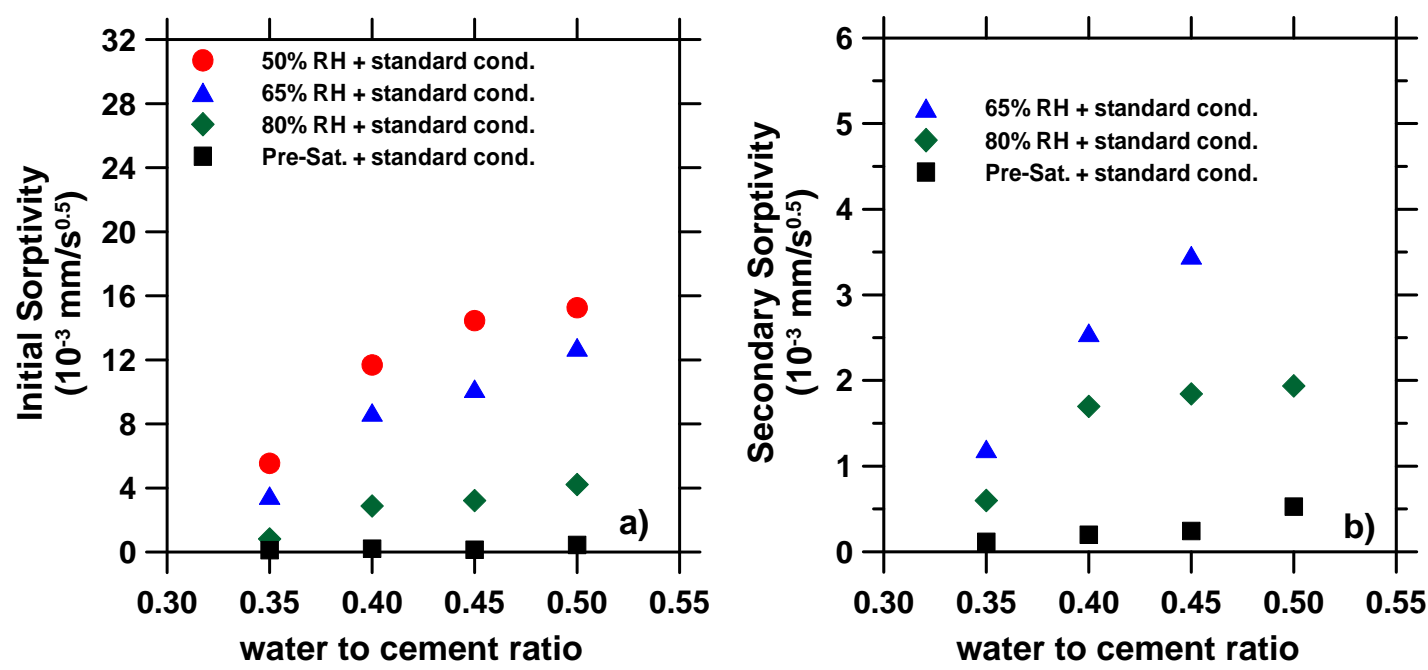

Figure. 4.11: Initial and Secondary Sorptivity on Mortars with Different Initial Moisture Content, Conditioned with the Procedure Established in ASTM C1585-04.

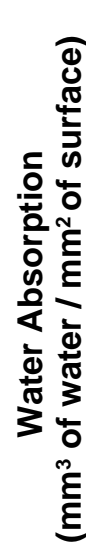

4

8 absorption of the aggregate in the samples. To better understand its effect, Figure 4.13

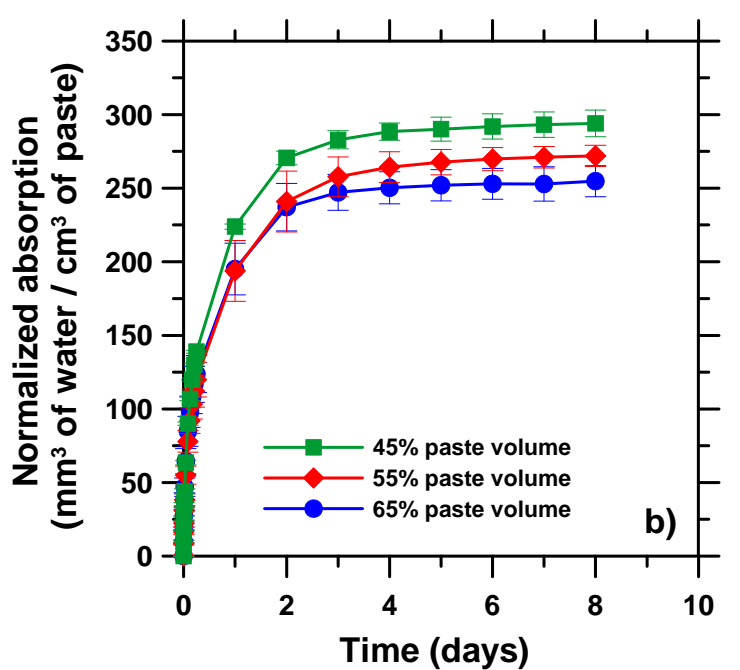

Figure 4.12: Water Absorption in Mortars Containing Different Volume of Aggregates: a) Normalized by Surface in Contact with Water, (b) Normalized by Volume of Paste.

Commonly water absorption is reported without considering the effect of the was calculated assuming five different sand absorptions $(0.0 \%, 0.6 \%, 1.2 \%, 1.8 \%$, and 
$12.4 \%$ ) to then subtract these values from the absorption in Figure $4.12 \mathrm{~b}$. When the sand

2 absorption is assumed to be $0.0 \%$, the resulting absorption at 8 days will be the same as

3 the absorption presented in Figure 4.12b. From Figure 4.13, it can be noticed that for the

4 assumed $1.8 \%$ sand absorption, the normalized water absorbed for the sample is the

5 same after 8 days, independent of the amount of aggregate in the sample.

6 Figure 4.14 shows a desorption isotherm for the sand used in these mixtures. It

7 can be noted that at $50 \%$ RH (humidity at which the samples were conditioned), the

8 amount of water on the sand is about $0.2 \%$. Considering that the aggregate used in this

9 study has a $24 \mathrm{~h}$ absorption of $1.8 \%$, the difference on water absorption of samples

10 containing different amounts of aggregate can be explained mainly by the amount of

11 water absorbed by the aggregates.

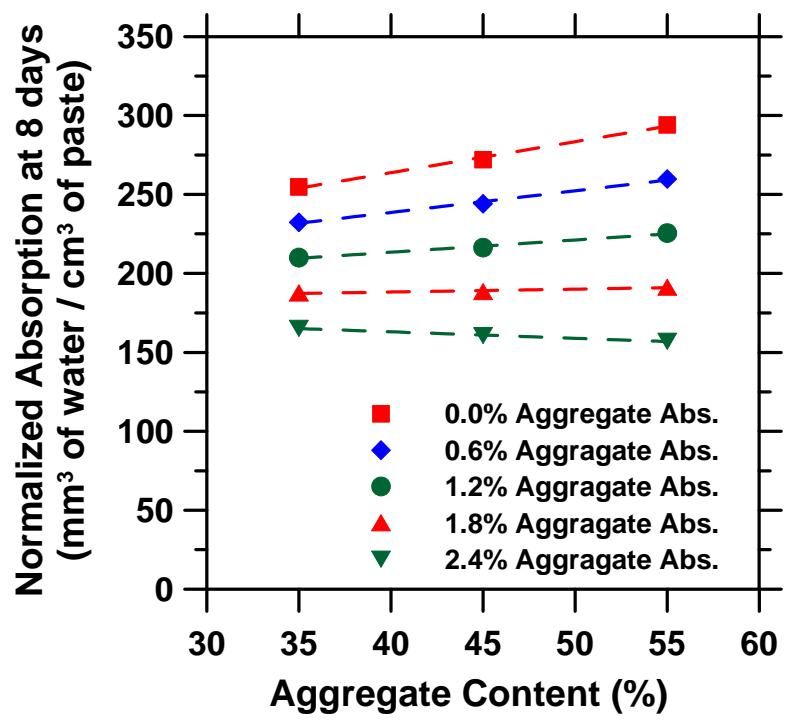

13 Figure 4.13: Water Absorption at 8 Days Normalized by Volume of Paste, Corrected by 14 Different Values of Aggregate Absorption. 


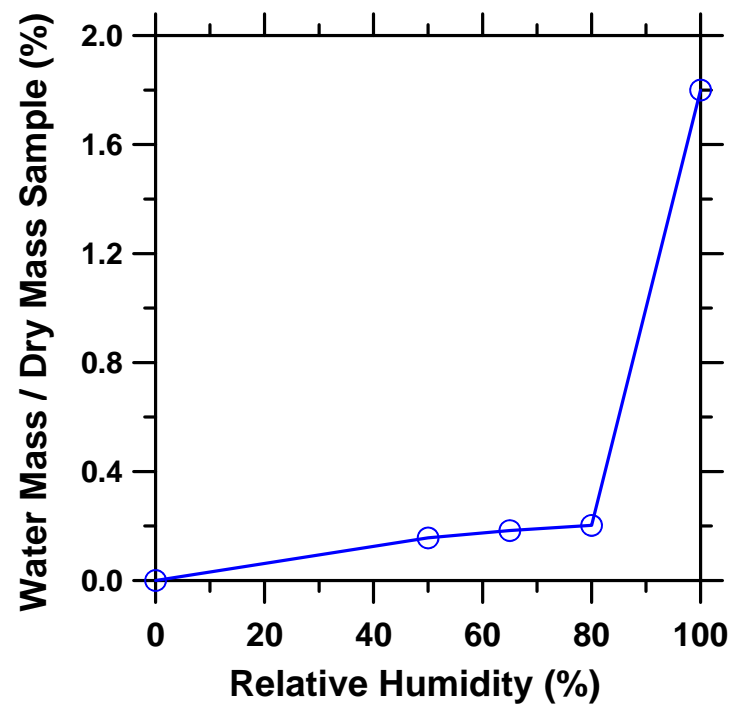

Figure 4.14: Desorption Isotherm for the Sand used in this Study.

4 This paper has described the absorption behavior of mortars conditioned at 5 different relative humidities. A summary of the general conclusions from the data 6 presented in this paper are:

7 - As was shown in previous works by Hall (1989), Hooton et al. (1993) and Martys and Ferraris (1997), the water absorption test is considerably affected by the

9 relative humidity of the samples before starting the test, which if not properly

10 accounted for can lead to a misunderstanding of the actual absorption behavior.

11 - Samples conditioned at $50 \%$ relative humidity can show a total absorption that is 12 approximately six times greater than similar samples conditioned at $80 \%$ relative 13 humidity. This is consistent with what can be expected from the desorption 14 curves. 
1 - Initial sorptivity, secondary sorptivity and total absorption at 8 days for samples

2 conditioned in chambers show a linear trend related to the $w / c$ and the relative

3 humidity at which samples were conditioned.

4 - Samples that are conditioned by drying in an oven at $105^{\circ} \mathrm{C}$ do not follow the 5 same trend as samples conditioned in other approaches. This is attributed to two

6 factors: 1) emptying of a wider range of pores, and 2) the potential for 7 microcracking.

8 - The conditioning procedure described in ASTM C1585-04 is not able to eliminate

9 the "moisture history" of the samples, and thus can lead to a misunderstanding of

10 the water absorption test results, especially in field samples which have obtained a

$11 \quad$ lower relative humidity.

12 - Comparing samples containing different volumes of aggregate can also lead to a 13 misunderstanding of the actual absorption behavior. Samples containing higher

14 volumes of cement paste will absorb more water. When the results are normalized

15 by the volume of cement paste, the sample containing lower volumes of cement

16 paste will absorb more water. However, this difference can be mainly explained

17 by the amount of water absorbed by the aggregates in the sample. 


\section{$\underline{5.1 \text { Overview }}$}

A series of wetting and drying tests were performed on concrete using different

5 aqueous solutions containing deicing salts. The rate of absorption was generally lower for

6 aqueous solutions containing deicing salts. In addition, less fluid was absorbed for

7 aqueous solutions containing deicing salts. The change in the rate of aqueous fluid

8 absorption was proportional to the square root of the ratio of surface tension and viscosity

9 of the absorbed fluid. Concrete that has been exposed to solutions containing deicer salts

10 show less mass loss during drying. Measures of equilibrium relative humidity over the

11 salt solutions are used to interpret drying behavior. Experimental data indicates that

12 concretes that have previously been exposed to deicing solutions can also exhibit reduced

13 rate of absorption, even if water is used as the fluid being absorbed.

\section{$\underline{5.2 \text { Introduction }}$}

15 Some jointed plain portland cement concrete pavements in freezing prone

16 climates have shown premature deterioration at the longitudinal and transverse joints.

17 While some have attributed this damage to a chemical attack, inadequate air entrainment,

18 poor mixture design, inadequate constituent materials, or poor construction practices; it is 
1 the hypothesis of the authors of this paper that this joint deterioration may be attributed,

2 at least in part, to preferential absorption of fluid at joints. This hypothesis was

3 developed based on observations from the field that show these deteriorated locations

4 frequently occurred at low spots in the pavement, where joint sealers were damaged,

5 where water has collected, or where the joint does not appear to have opened thereby

6 trapping water (Weiss and Nantung 2006). Preferential fluid ingress at joints could

7 increase a variety of damage mechanisms including deleterious chemical reactions,

8 crystallization pressure, or freeze thaw damage that may degrade the concrete. To fully

9 evaluate fluid ingress at the joints it is essential that the wetting and drying behavior of

10 concrete is evaluated using aqueous solutions containing deicing salts.

11 This work is limited in scope as it considers only the ingress of aqueous solutions

12 over short time periods and does not explicitly consider any chemical reaction that occurs

13 between the aqueous solution and the concrete or any long-term effects. This information

14 is intended to provide reference for those developing tests to evaluate potential deicer-

15 concrete interactions (Shi et al. 2010), for developing tests on fluid absorption, for

16 evaluating fluid absorption in concrete (Hong and Hooton 1999), for input parameters in

17 computer simulation of fluid ingress at joints (Pour-Ghaz et al. 2009), and for potential

18 approaches to limit joint deterioration like penetrating sealers for possible use in concrete

19 pavements (Coates et al. 2009).

Fluid absorption is a frequently used test to provide an indication of the durability

22 of concrete systems since it is simple to perform. Several standard tests exist for 
1 measuring water absorption including ASTM C 1585-04 (ASTM 2004), BS 1881-99

2 (British Standard Institute 1990), and ASTM D6489-99 (ASTM 2006). While the

3 concept behind these tests is very similar, there are differences in how the samples are

4 conditioned, treated, and tested. In each of these tests water is typically used as the fluid

5 that is being absorbed. Hall (1997) discusses that water can interact with the cement

6 matrix adding complexity to the interpretation of results. To overcome some of these

7 limitations or to indicate how absorption can be reduced by fluid composition other

8 solutions have been tested (Hall et al. 1997, Hall and Hoff 2002, Weiss 1999, Bentz et al.

9 2009, Sant et al. 2010).

10 MacInnis and Nathawad (1980) assessed the absorption of an aqueous solution

11 consisting of a $\mathrm{NaCl}$ deicing salt and reported a decrease in absorption. Sutter et al.

12 (2008) reported that sorptivity decreased from highest to lowest in the order of water,

$13 \mathrm{NaCl}, \mathrm{CaCl}_{2}$ and $\mathrm{MgCl}_{2}$. Similar data has recently been observed by Janusz (2010). As a

14 result, it can be observed that concrete exposed to deicing salt solutions absorb fluid at a

15 slower rate than they would absorb water; however the previous work has not related this

16 behavior to the fluid properties or described the influence of salt concentration or

17 properties of the aqueous solution.

18 The results of one-dimensional fluid absorption tests (assuming negligible

19 gravitational effects) are typically reported as the cumulative water absorbed per surface

20 area (surface from which water is absorbed) versus the square root of wetting time.

21 Equation 5.1 can be used to describe the water absorption (total volume of fluid

22 absorbed) and the sorptivity (related to the rate of absorption). 
$1=S \tau^{1 / 2}$

2 where $i\left[\mathrm{~mm}^{3} / \mathrm{mm}^{2}\right]$ is the cumulative water absorption, $S\left[\mathrm{~mm} / \mathrm{s}^{1 / 2}\right]$ is the sorptivity, and $3 \tau[\mathrm{s}]$ is the elapsed time. Hall (1997) proposed that the diffusion would scale proportionately with the ratio 5 of surface tension $(\gamma)$ and viscosity $(\eta)$ of the fluid. Hall further related this to sorptivity 6 since sorptivity is related to the square root of diffusion. Kelham (1988) derived an 7 expression for fluid absorption (Equation 5.2) that shows the relationship between depth 8 of penetration and the square root of the ratio of surface tension and viscosity.

$$
x(\tau)=\sqrt{\frac{4 k \gamma \cos (\theta) \tau}{p \eta r}}
$$

9 where $x(\tau)[\mathrm{mm}]$ is the penetration depth, $\gamma[\mathrm{N} / \mathrm{mm}]$ is the surface tension, $\theta[\mathrm{rad}]$ is the 10 liquid-solid contact angle, $p[\sim]$ is the porosity of the medium, $r[\mathrm{~mm}]$ is the pore radius, $11 k\left[\mathrm{~mm}^{2}\right]$ is the intrinsic permeability of the material, and $\eta[\mathrm{Pa} . \mathrm{s}]$ is the viscosity of fluid. 12 An expression similar to equation 3 was derived by Scherer and Wheeler (2009) for stone 13 consolidates.

14 Previous research using organic fluids have been noticed to have an absorption 15 rate that scales proportionally with the square root of the ratio of surface tension and 16 viscosity of the fluid $\left((\gamma / \eta)^{1 / 2}\right)$. This work will use this approach to attempt to interpret 17 results from absorption tests that used aqueous solutions containing deicing salts. 
2 5.4.1 Experimental Program of Wetting and Drying of Concrete with Deicing Solutions

3 The concrete mixture that was used for these tests was a typical INDOT class C

4 bridge deck concrete. The mixture proportions of this concrete are shown in Table 5.1.

5 The fresh air content was $5.7 \%$ measured according to ASTM C231-09 (2009). The

6 hardened air content of the concrete was $4.4 \%$ as assessed using an automated optical

7 scanning approach (Castro et al. 2010) based on the approach of Peterson et al. (2001).

8 Table 5.1: Mixture Proportions Assuming Saturated Surface Dry (SSD) Conditions

\begin{tabular}{|c|c|}
\hline Material & Mass \\
\hline Cement $\left(\mathrm{kg} / \mathrm{m}^{3}\right)$ & 316 \\
\hline Class C Fly Ash $(\mathrm{kg} / \mathrm{m} 3)$ & 60 \\
\hline Water $\left(\mathrm{kg} / \mathrm{m}^{3}\right)$ & 150 \\
\hline Fine Aggregate $\left(\mathrm{kg} / \mathrm{m}^{3}\right)$ & 736 \\
\hline Coarse Aggregate $\left(\mathrm{kg} / \mathrm{m}^{3}\right)$ & 1049 \\
\hline Air Entrained Admix. $(\mathrm{ml} / 100 \mathrm{~kg} \mathrm{cem}$. materials) & 20 \\
\hline High Range Water Reducer Admix. $(\mathrm{ml} / \mathbf{1 0 0} \mathrm{kg}$ cem. materials) & 456 \\
\hline Retarder Admixture $(\mathrm{ml} / 100 \mathrm{~kg} \mathrm{cem}$. materials) & 98 \\
\hline
\end{tabular}

10 The concrete was produced in a central mix plant and discharged from a ready

11 mix concrete truck before the concrete was sampled. A series of $100 \mathrm{~mm} \times 200 \mathrm{~mm}$

12 cylinders were cast. After one day of curing, the cylinders were demolded and sealed in

13 double plastic bags at $23 \pm 0.5^{\circ} \mathrm{C}$ until the samples reached an age of $28 \mathrm{~d}$. After 28 days

14 of curing the cylinders were removed from bags and three $50 \mathrm{~mm} \pm 2 \mathrm{~mm}$ thick samples

15 were cut from the central portion of each cylinder with a wet saw. 
Two different series were used to condition the concrete samples. The first series

2 evaluated the role of different conditioning regimes on the absorption of water using

3 three samples for each curing condition. The samples in the first series were conditioned

4 in five different ways (ASTM, oven-dry, $50 \% \mathrm{RH}, 65 \% \mathrm{RH}$ and $80 \% \mathrm{RH}$ ). A portion

5 of the samples were conditioned following the procedure in ASTM C1585-04 (after

6 conditioning the samples for 12 months at $50 \% \mathrm{RH}$ they were vacuum saturated for $24 \mathrm{~h}$

7 and then exposed to ASTM C1585-04 conditions). The remainder of samples were

8 conditioned in four different ways: 1) at $80 \% \mathrm{RH}$ for 12 months, 2) at $65 \%$ for 12

9 months, 3) at $50 \%$ for 12 months, and 4) oven dried. The second series of samples

10 consists of concrete samples that were dried at $50 \pm 2 \% \mathrm{RH}, 23 \pm 0.5{ }^{\circ} \mathrm{C}$ for 36 months

11 and two samples were tested for each curing condition.

12 To prepare the specimens for fluid absorption testing the sides of the sample were

13 sealed with epoxy. After the epoxy had hardened the top surface was covered with

14 plastic to avoid evaporation from the sample during testing.

15 The absorption test involves recording incremental mass change measurements

16 during the first six hours after the sample comes in contact with the fluid and

17 subsequently taking one measurement every day for the next eight days. The amount of

18 absorbed fluid is normalized by the cross-section area of the specimen exposed to the

19 fluid using Equation 5.3. 


$$
\mathrm{i}=\frac{\mathrm{m}_{\mathrm{t}}}{(\mathrm{a} \cdot \rho)}
$$

1 where: $\mathrm{i}\left(\mathrm{mm}^{3} / \mathrm{mm}^{2}\right)$ is the normalized absorbed fluid, $\mathrm{m}_{\mathrm{t}}(\mathrm{g})$ is the change in specimen

2 mass at time $\mathrm{t} ; \mathrm{a}\left(\mathrm{mm}^{2}\right)$ is the area of the specimen exposed to the fluid (i.e., that of the

3 bottom face), and $\rho\left(\mathrm{g} / \mathrm{mm}^{3}\right)$ is the density of the absorbed fluid (this is provided in

4 greater detail later in the paper). These absorption measurements are then plotted as a

5 function of the square root of time. The sorptivity is the slope of this graph.

6 The second series of samples were tested using seven different fluids. Their

7 composition primarily based upon, one of three different industrially available deicing

8 products, either $\mathrm{NaCl}, \mathrm{MgCl}_{2}$ or $\mathrm{CaCl}_{2}$. A low concentration was used for each salt as

9 well as a higher concentration that was selected to be near the eutectic composition for

10 each salt. De-ionized water was also used as a reference fluid.

11 5.4.2 Experimental Results from Wetting with Different Conditioning Methods

12 Figure 5.1 shows the results from water absorption tests performed on the first

13 series of samples that were conditioned with different environmental conditions as

14 mentioned earlier (ASTM C1585-04 accelerated conditioning, $80 \% \mathrm{RH}, 65 \%$ RH, $50 \%$

$15 \mathrm{RH}$ and oven drying). It should be remembered that these samples were conditioned for

1612 months while the remainder of the samples discussed in this paper were conditioned at

$1750 \% \mathrm{RH}$ for a much longer time. Sample preparation has an enormous impact on the 
1 water absorption results as more severe drying enables a greater volume of water to be 2 absorbed during the test.

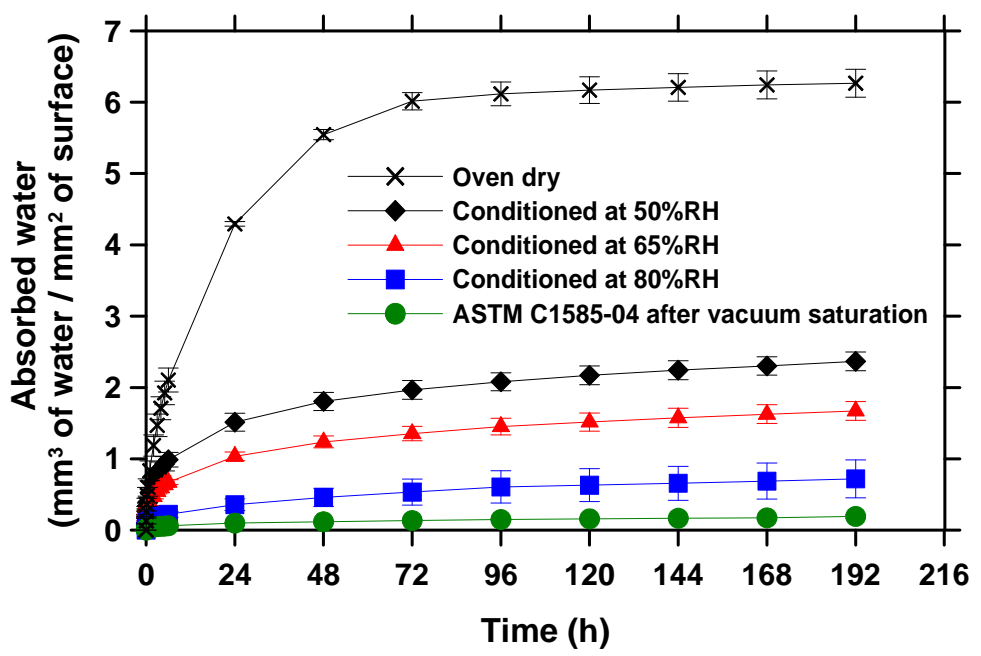

8 (for concrete at $50 \% \mathrm{RH}$ for a longer conditioning time than the samples in Figure 5.1).

9 It can be seen that even though the concrete that is used for all the tests in Figure 5.2 has

10 the same conditioning and exposure conditions, the volume of solution absorbed by each

11 material is dependent on the deicing salt solution and the concentration of the deicing salt

12 solution that was absorbed. The sample with the low concentration of $\mathrm{NaCl}$ showed a

13 slight increase in the rate of absorption (as compared with water) as well as the amount of

14 fluid absorbed. This is consistent with the data reported by MacInnis and Nathawad

15 (1980). The absorption of all the other fluids was reduced when compared with water. 
1 As a result it can be concluded that in general as the salt concentration increased a

2 reduced rate of absorption and reduced total absorption was observed. Further work is

3 needed to examine lower concentrations for $\mathrm{NaCl}$ to ascertain why a slight increase is

4 typically reported.

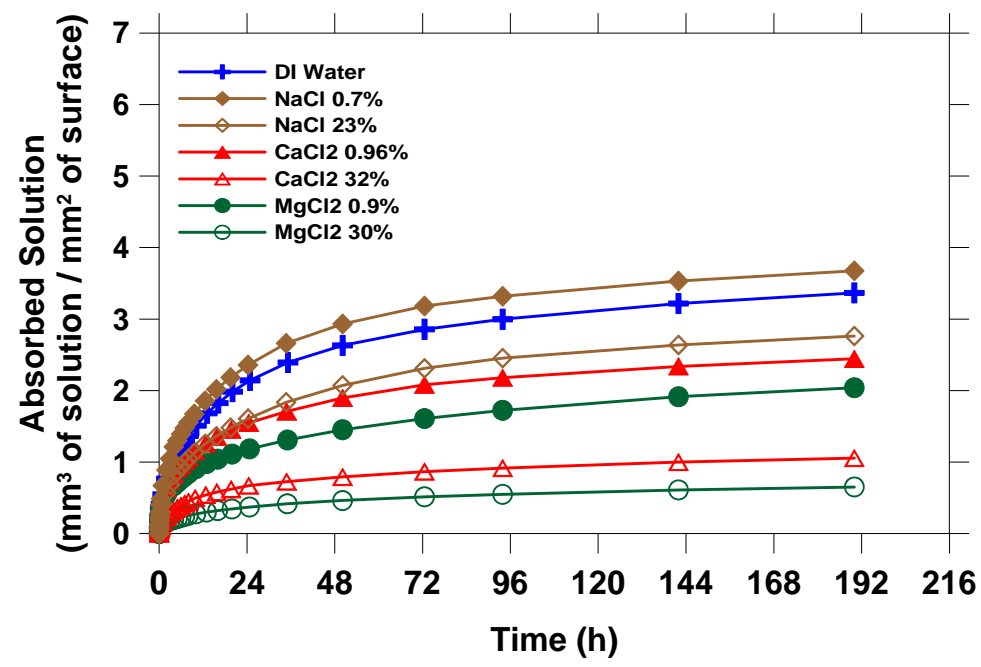

5

6

7

$950 \pm 2 \% \mathrm{RH}, 23 \pm 0.5{ }^{\circ} \mathrm{C}$ for seven days. The samples were kept in the same one-faced

10 exposed condition for the drying test; however, the exposed surface that was facing down

11 in the absorption testing was placed facing up to simulate drying from the top. During the

12 drying test the mass of the samples was recorded at regular intervals.

13 Figure 5.3 shows the volume of water loss during the drying period. It is

14 important to note that the drying test will result in only the water portion of the solution

15 being evaporated from the system leaving the salt to become more concentrated in the 
1 solution before it eventually precipitates out. It can be noticed that as the concentration

2 of deicing solution was increased the mass loss during drying decreased. This was

3 particularly evident in the high concentration solutions which showed nearly no mass loss

4 or even a slight gain during drying.

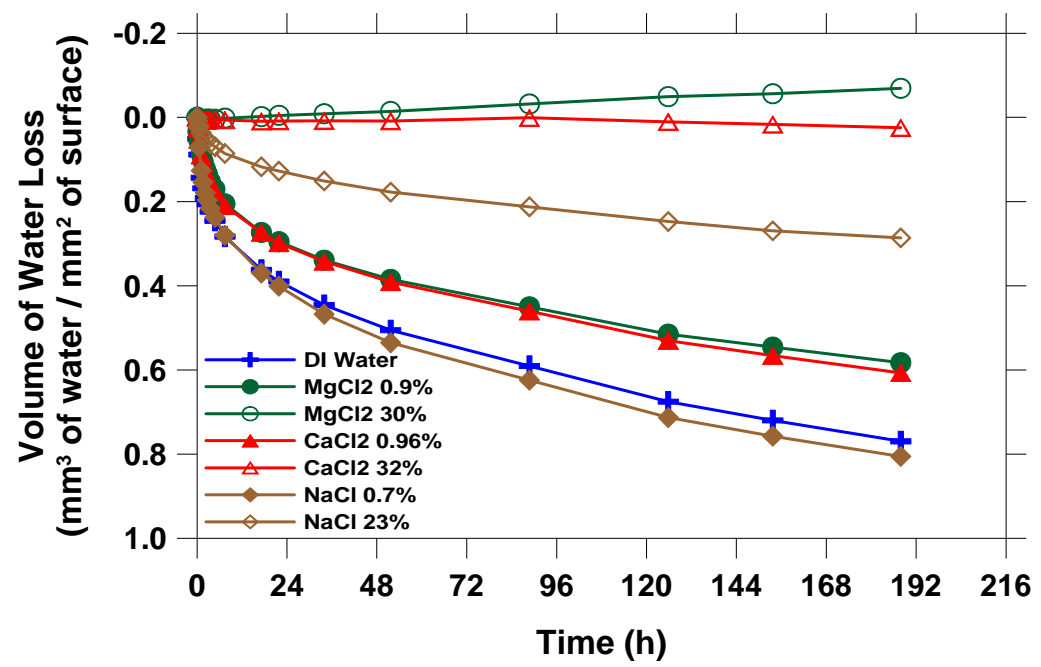

6 Figure 5.3: Drying of Concrete Prewetted with Different Salt Solutions as a Function of $7 \quad$ Time (Typical Standard Deviation less than $0.03 \mathrm{~mm}^{3}$ water $/ \mathrm{mm}^{2}$ )

8 5.4.4 Experimental Results from Wetting Previously Exposed to Deicing Solutions

9 The samples that were first tested for absorption of different aqueous solutions,

10 then tested for drying, were then oven dried so that a second fluid absorption test could

11 be performed. The second fluid absorption test however used only de-ionized water as

12 the fluid that was being absorbed.

13 To prepare the samples for the second wetting test they were placed in an oven at $14105 \pm 2{ }^{\circ} \mathrm{C}$ until the difference between any two $24 \mathrm{~h}$ apart successive mass 15 measurements were less than $0.5 \%$ (i.e., approximately 5 days). It is important to note 
1 that this drying process will evaporate only the water portion of the solution pre-

2 absorbed, leaving salt in the pores. Since these samples were oven dried, their absorption

3 rates and absorbed water are not comparable with any previous tests.

$4 \quad$ Figure 5.4 shows the results for this second absorption test. It can be seen by

5 comparing the results to the results in Figure 5.2 that the behavior of the samples was

6 dependent on the deicing solutions and the concentrations of deicing solutions used in the

7 first wetting test. These results are a clear indication that the history of the samples

8 affects the results of fluid absorption. This suggests when sorption testing is preformed

9 on field concretes some understanding of the admixtures or salts that remain in the pore

10 system is needed to fully interpret the results.

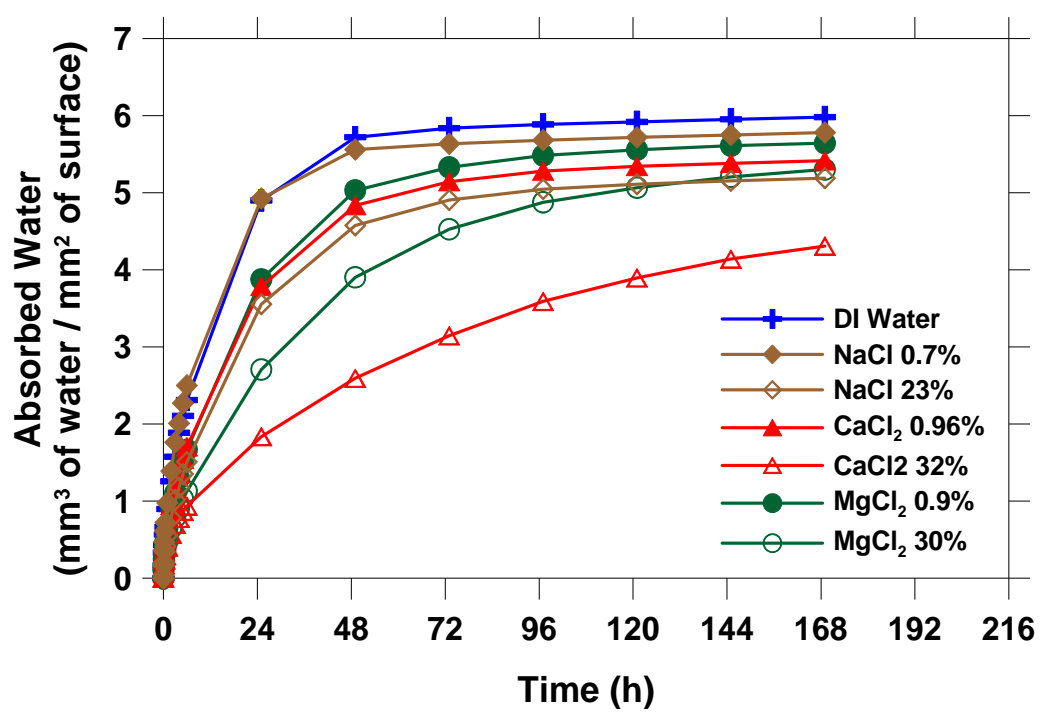

12 Figure 5.4: Volume of De-ionized Water Absorbed by Concrete as a Function of Time in 13 the Second Fluid Absorption Test (Fluid from the Original Test is Shown in the Caption) 


\subsubsection{Drying of Mortars Saturated with Different Deicing Salts}

Moisture desorption is an established technique for evaluating the effect of

3 moisture loss at a given humidity for a material. A TA Q5000 SA moisture sorption

4 analyzer was used to carefully control temperature and humidity. Mortar samples were

5 prepared $(w / c=0.42$ and $55 \%$ aggregate by volume) and cast in a cylindrical mold with

6 a $34 \mathrm{~mm}$ diameter and $50 \mathrm{~mm}$ height. At an age of 28 days the specimens were

7 demolded and $34 \mathrm{~mm}$ diameter $0.8 \pm 0.05 \mathrm{~mm}$ thick slices were taken from the middle of

8 the samples. The samples were dried under controlled conditions (at $23 \pm 0.1{ }^{\circ} \mathrm{C}$ and 50

$9 \pm 1 \% \mathrm{RH})$ in a $\mathrm{CO}_{2}$ free chamber until they reach mass equilibrium. Then, samples were

10 submerged for a minimum of 5 days in aqueous solutions with $23 \% \mathrm{NaCl}, 32 \% \mathrm{CaCl}_{2}$,

11 and $30 \% \mathrm{MgCl}_{2}$ by mass.

12 For the samples submerged in $\mathrm{NaCl}, \mathrm{CaCl}_{2}$, and $\mathrm{MgCl}_{2}$ solution,s a $50 \mathrm{mg}$ to 70

$13 \mathrm{mg}$ piece of sample was placed in a tared quartz pan after a minimum of 5 days of

14 submersion. The pan containing the sample was then suspended from the balance $( \pm$

$150.001 \mathrm{mg}$ accuracy) and placed in the relative humidity chamber to equilibrate at $23.0 \pm$

$160.1{ }^{\circ} \mathrm{C}$ and $97.5 \pm 0.1 \% \mathrm{RH}$ for up to $96 \mathrm{~h}$ or until the sample had achieved a stable mass

17 (less than an $0.001 \%$ mass change/15 minutes).

18 Then, the relative humidity was reduced to reach $95 \%$. After the sample mass

19 equilibrated, the relative humidity in the chamber was changed in $10 \% \mathrm{RH}$ steps to $55 \%$

$20 \mathrm{RH}$, allowing the sample to attempt to equilibrate ( $12 \mathrm{~h}$ or $0.01 \%$ change in mass over 15

21 minutes) at each new humidity. After equilibrating at $55 \% \mathrm{RH}$ the samples were dried to

$220 \% \mathrm{RH}$. For the sample submerged in de-ionized water the procedure was similar, but 
1 the relative humidity was reduced in $5 \%$ steps from $97.5 \%$ to $2.5 \%$, and then reduced to $20 \% \mathrm{RH}$.

3 Figure 5.5 shows the plot of mass change as a function of time for the mortar

4 saturated in de-ionized water. The sample soaked in water can be seen to lose mass with

5 the decrease of RH. For this system, when the environment is below $100 \% \mathrm{RH}$, water

6 will move from the pores to outside of the sample and classical drying behavior is

7 observed. The maximum mass of the sample is $8.5 \%$ higher than the mass of the oven

8 dry sample.

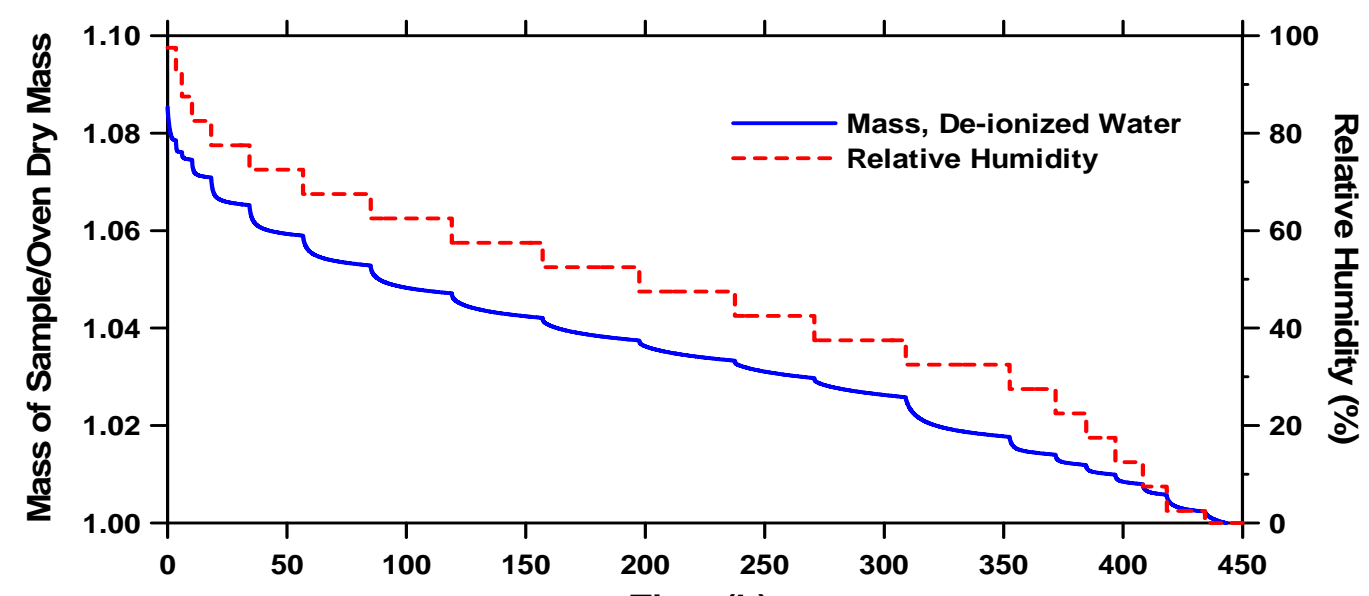

9

10 Figure 5.5: Mass Change at Decreasing RH for Samples Containing De-ionized Water

11 Figure 5.6 shows a plot of mass change for the mortar samples submerged in

12 aqueous solutions of $23 \% \mathrm{NaCl}, 32 \% \mathrm{CaCl}_{2}$, and $30 \% \mathrm{MgCl}_{2}$. It can be observed that

13 initially upon placement in the testing chamber at $97.5 \%$ relative humidity the mass of

14 the sample increases for the first $96 \mathrm{~h}$ until the relative humidity of the chamber is

15 changed. The samples absorb water during this time of preconditioning, with values 
1 much higher than the $8.5 \%$ increase in mass of the sample with de-ionized water as

2 compared with the oven dry sample.

3 The sample loses weight as the relative humidity is decreased however it should

4 be noted that the sample mass does not decrease to below the initial mass obtained from

5 soaking the sample in the deicing solution until relative humidity was decreased below 85

$6 \%, 55 \%$ and $55 \%$ for $\mathrm{NaCl}, \mathrm{CaCl}_{2}$ and $\mathrm{MgCl}_{2}$ respectively. This will be compared with

7 the equilibrium relative humidity of the salt solution later in the paper. 

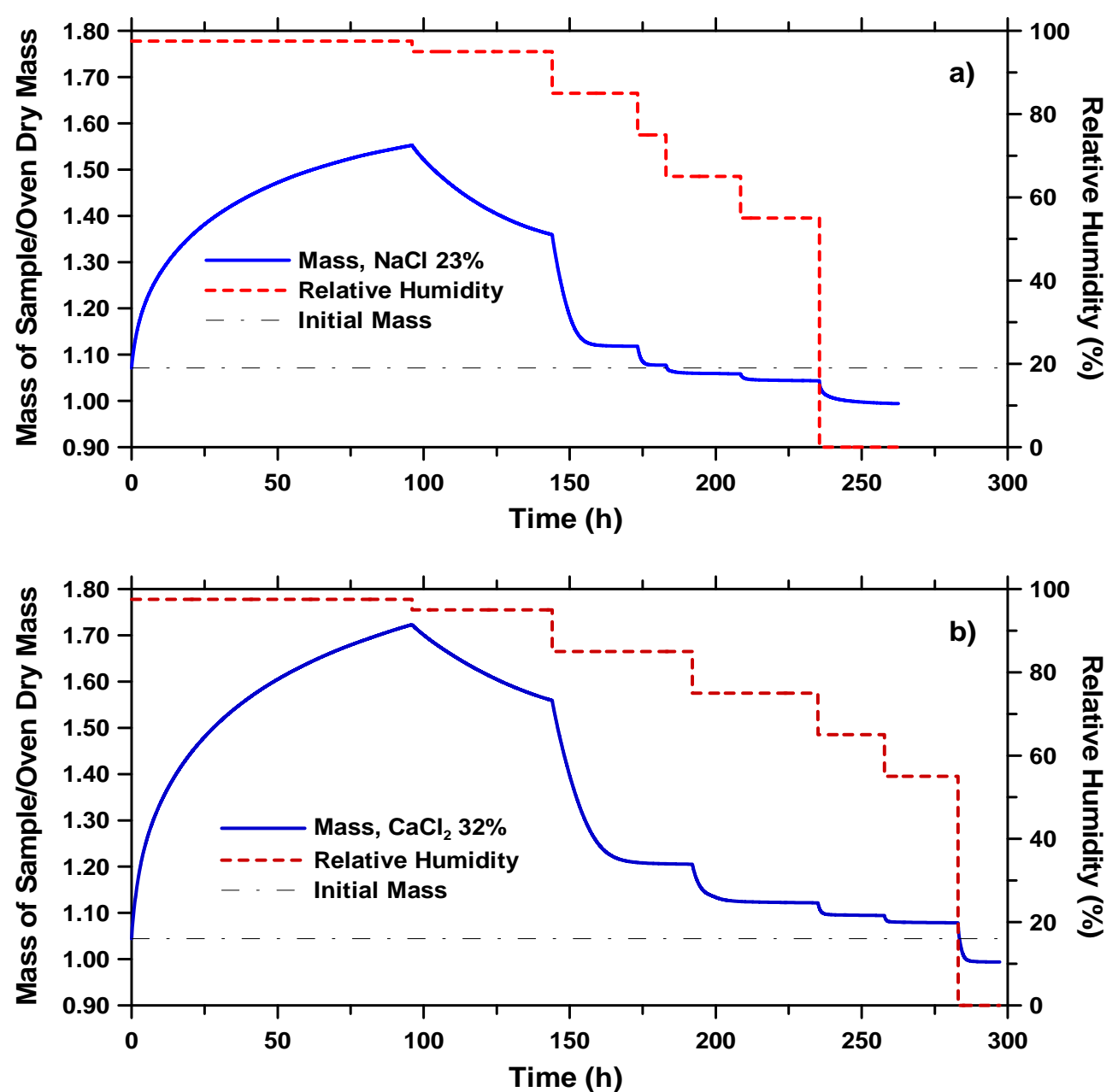

2

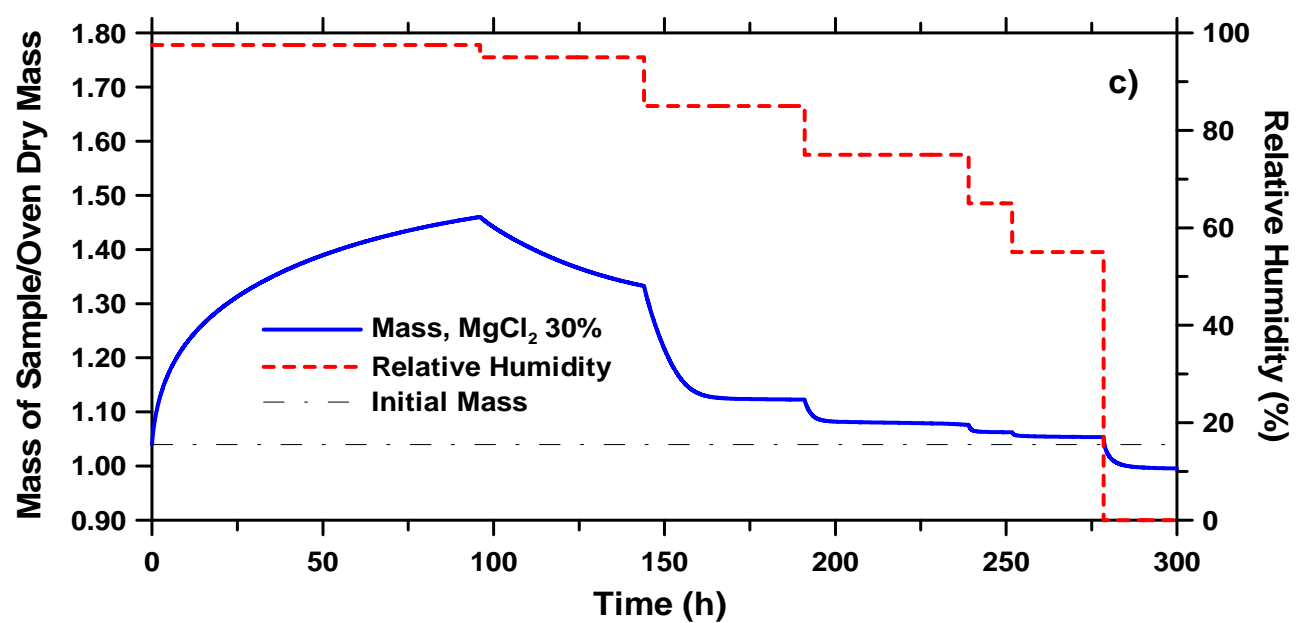

4 Figure 5.6: Mass Change for Samples Submerged in Aqueous Solutions Containing 5 Deicing Salts: (a) $\mathrm{NaCl} 23 \%$ (b) $\mathrm{CaCl}_{2} 32 \%$ and (c) $\mathrm{MgCl}_{2} 30 \%$ 


\subsection{Properties of Deicing Salt Solutions}

Physical properties of pure solutions were gathered from literature and compared

3 with measured values for the industrially available deicing solutions tested in this

4 research, and they are provided here for convenience in one location. The properties of

5 the deicing solutions will be used in interpreting the wetting and drying results. This

6 section is divided into four sections. The first three sections describe the influence of the

7 deicing solutions in terms of surface tension, viscosity, and equilibrium relative humidity

8 over the aqueous solution. The fourth section describes the specific gravity of the

9 solution as a function of concentration as this is used to determine the volume of solution

10 absorbed during the absorption test.

\subsubsection{Surface Tension of Deicing Salt Solutions}

12 Figure 5.7a shows surface tension measurements at different concentrations for

13 the three solutions used in this research: $\mathrm{NaCl}, \mathrm{CaCl}_{2}, \mathrm{MgCl}_{2}$. The surface tension for

$14 \mathrm{NaCl}$ was obtained from (Hall and Hoff, 2002), $\mathrm{CaCl}_{2}$ from (Conde, 2004) and $\mathrm{MgCl}_{2}$

15 from (Phang and Stokes, 1980). A Du Noüy Ring Tensiometer KRÜSS was used with a

16 resolution of $0.1 \mathrm{mN} / \mathrm{m}$ for the industrial deicers tested in this study. The tensiometer

17 was cleaned between measurements following ASTM D971-04 (ASTM, 2004). The

18 tensiometer was first calibrated using de-ionized water, which provided a value of $71.0 \mathrm{x}$

$1910^{-6} \mathrm{~N} / \mathrm{mm}$. A series of three measurements were performed for each solution, with the

20 average reported.

21 The closed points in Figure 5.7a are the values measured for the industrially

22 available solutions. The lines represent values taken from literature for pure salt 
1 solutions at different mass concentrations. While the general trends are consistent,

2 differences between the solutions containing industrial deicing salts and literature values

3 may be due to impurities or other additives however further work is needed to examine

4 this in greater detail.

\subsubsection{Viscosity of Deicing Salt Solutions}

Figure $5.7 \mathrm{~b}$ shows a comparison of the viscosities for the solutions used in this

7 research between pure solutions taken from literature and measurements of the deicing

8 solutions. Viscosity measurements for the industrial deicers were performed on the salt

9 solutions using an Anton-Parr rheometer, model Physica MCR 301. The rheometer kept

10 the solution being tested at $23.0 \pm 0.02{ }^{\circ} \mathrm{C}$ and from the torque applied to the fluid that

11 causes a shear from which the viscosity can be found. Calibration of the device was

12 performed using a reference standard.

13 The dashed lines presented are viscosities at different concentrations and are

14 taken from literature (Hall and Hoff, 2002; Conde, 2004; Phang and Stokes, 1980, Afsai

15 et al., 1989), while the points represent measured viscosities of the industrially available

16 solutions. Again, differences between literature values and those of the solutions

17 measured can be explained by differences in possible additions or chemistries of the 18 industrial deicers. 

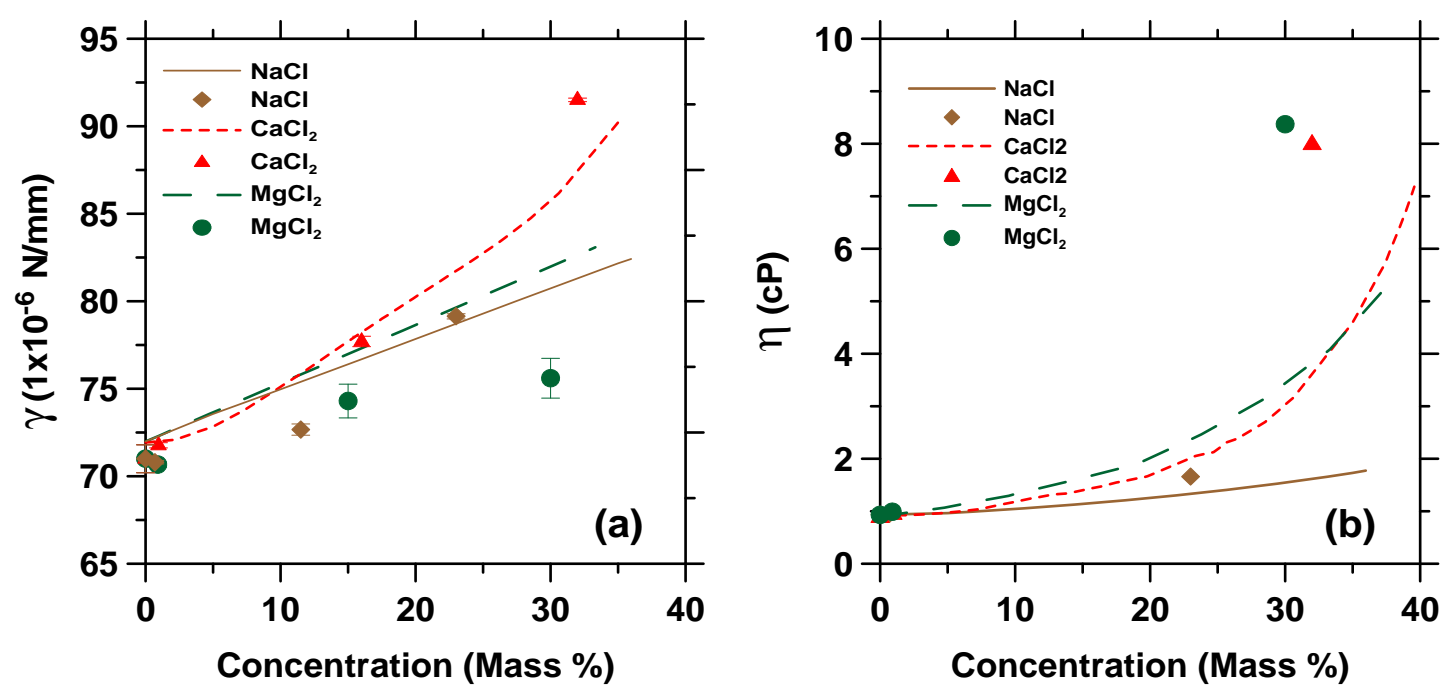

2
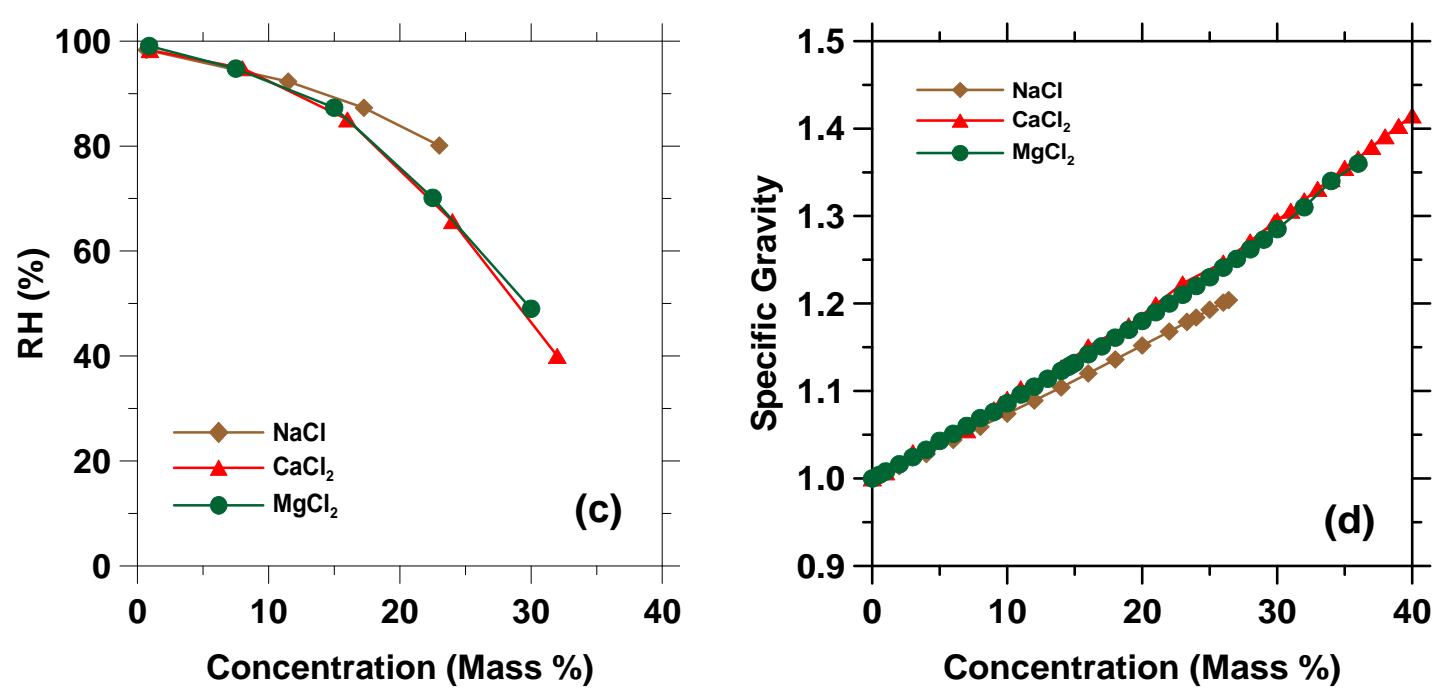

4 Figure 5.7: Properties of Deicing Salts at 23-25 ${ }^{\circ}$ : : (a) Surface Tension (b) Viscosity (c) 5 Relative Humidty (d) Specific Gravity (Rosenburgh 2010, unpublished data) 
11 for $\mathrm{NaCl}$ (Greenspan, 1977), $33.0 \% \mathrm{RH}$ for $\mathrm{MgCl}_{2}$ (Greenspan, 1977) and $22 \%$ for

$12 \mathrm{CaCl}_{2}$ (Conde, 2004).

15 of concentration. The specific gravity of the solution increases with concentration. The

$16 \mathrm{CaCl}_{2}$ and $\mathrm{MgCl}_{2}$ increase at very similar rates with an increase in concentration, while

17 the $\mathrm{NaCl}$ increases slightly less than the $\mathrm{CaCl}_{2}$ and $\mathrm{MgCl}_{2}$ (i.e., $25 \%$ less increase with

18 concentration). This may be attributed to the colligative properties of solutions

19 (Diamond, 2010, Personal Communication). 
Equation 5.3 showed that the rate of absorption was related to the square root of

5 surface tension and viscosity. Figure 5.8 plots the square root of the ratio of surface

6 tension and viscosity versus mass concentration of salt. Pure salt solutions are shown as

7 lines while industrial deicing solutions are presented as solid points, and the open points

8 represent the measured sorption response of concrete (i.e., salt sorptivity/water sorptivity)

9 from Figure 5.2. Figure 5.8 confirms that as the solution concentration increases, the rate

10 of fluid absorption (i.e., sorptivity) decreases. Further, while the properties of pure

11 solutions may not exactly represent the response of industrially available deicing

12 solutions they do provide a comparable trend. Reasonable agreement is seen between the

13 measured sorption and square root of the ratio of surface tension and viscosity the

14 measured properties. Additional work is currently being performed to extend these

15 results to a wide range of temperatures. 


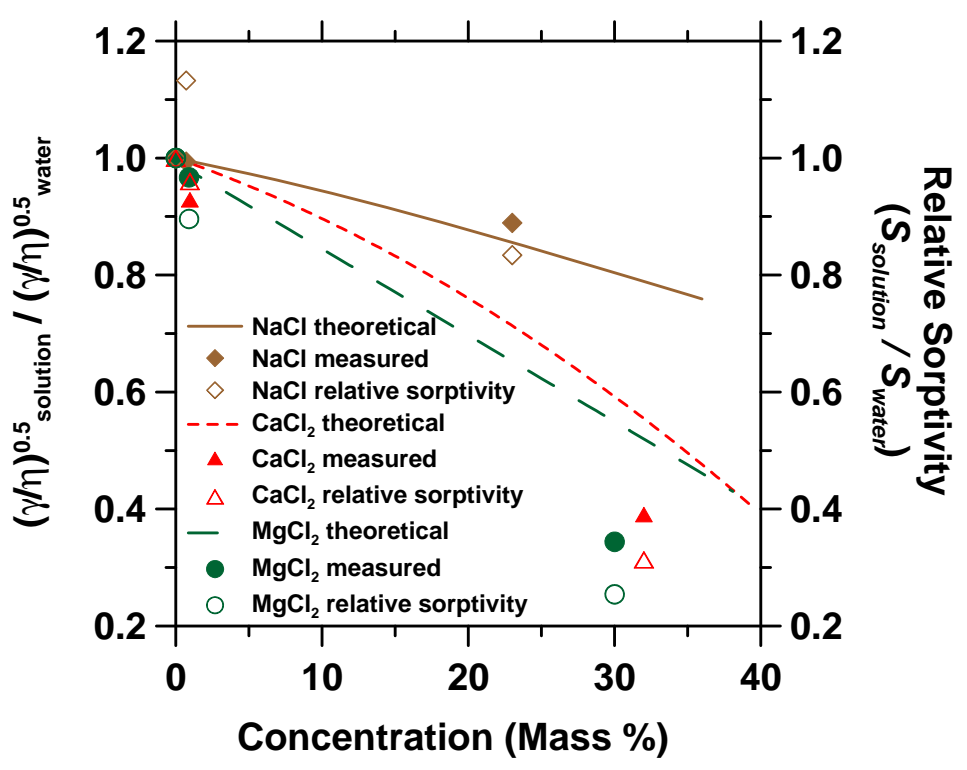

Figure 5.8: Relative Sorptivity for Deicing Solutions

5 drying. When de-ionized water was used as the absorbed fluid, the amount of fluid that

6 was evaporated from the sample after eight days was $0.8 \mathrm{~mm}^{3} / \mathrm{mm}^{2}$. In contrast, it took

7 just two hours for samples to absorb the same amount of fluid. These differences are

8 even larger when salt solutions were used as the absorbed fluid. When $\mathrm{MgCl}_{2}$ solution

9 was used as the absorbed fluid, the amount of fluid that was evaporated from the sample

10 after eight days was $0.07 \mathrm{~mm}^{3} / \mathrm{mm}^{2}$, but it took just ten minutes for the samples to absorb

11 the same amount of fluid.

12 This is important as it suggests that field concrete may be more susceptible to

13 increasing its level of saturation over time rather than drying out. Further, it shows that

14 laboratory tests that use equal times for drying and wetting increase the saturation level of

15 the concrete over time. Researchers (Wang et al. 2005) observed an increase in sample 
1 mass during wetting and drying cycling with deicers which was attributed to

2 microcracking; however an increase in mass would be consistent with the wetting and

3 behavior observed in this paper.

$4 \quad$ 5.6.3 Reduced Drying with Salt Solutions - The Role of Solution Equilibrium Humidity

$5 \quad$ The relative humidity of different salt solutions presented in Figure $5.7 \mathrm{~b}$ help to

6 understand the results from the drying tests. The equilibrium relative humidity for the

$723 \% \mathrm{NaCl}, 32 \% \mathrm{CaCl}_{2}$ and $30 \% \mathrm{MgCl}_{2}$ solutions are $80 \%, 40 \%$ and $50 \%$ respectively.

8 When the samples are placed in an environment with a relative humidity that is greater

9 than or equivalent to the approximate equilibrium relative humidity over the aqueous

10 solution in the pores water will not be lost (Figure 5.3) and the sample can actually gain

11 mass (Figure 5.3 and 5.6). This can be seen by the thinner (dashed lines in Figure 5.6),

12 which show the initial mass of the sample after it has been submerged in a aqueous

13 solution for over 5 days. At relative humidity higher than the equilibrium of the aqueous

14 salt solution the samples will increase in mass. At relative humidities where the

15 environment is less than the equilibrium humidity over the salt solution, the samples will

16 be expected to decrease in mass. The drying behavior of systems containing concentrated

17 aqueous solutions of deicing salts is complex and requires additional research.

\subsubsection{Effect of Solution on Rewetting}

19 When samples of concrete that were previously exposed to deicing solutions were 20 rewet with water they had an absorption and rate of absorption that depended on the 
1 history of the specimens (Figure 5.4). The absorption of water can be $30 \%$ to $50 \%$ less

2 in specimens that were exposed to deicing solutions at some point in their lives. This is

3 an important, yet subtle, factor to understand. This is important since absorption tests of

4 field concrete may be mistakenly interpreted by relating the reduction in sorption to pore

5 filling or delayed sorption microcracking. Both of these observations (lower sorptivity

6 and delayed sorption) are consistent with data here for samples that did not have reduced

7 porosity or differences in sample damage.

\section{$\underline{5.7 \text { Summary and Conclusions }}$}

9 This paper has reported experimental results from aqueous solution absorption

10 measurements and drying measurements for concrete in the presence of deicing solutions.

11 The following observations can be made. First, the absorption of fluid in concrete

12 depends on the drying environment used to condition the samples. Samples stored at a

13 lower RH absorbed a greater volume of fluid. Second, it was observed that the deicing

14 solutions reduce the rate of fluid absorption. This reduction can be related to the square

15 root of the ratio of surface tension and viscosity (Hall and Hoff 2002). Third, the time

16 scale between drying and wetting is different and concrete is more likely to become

17 preferentially increasingly wet over time. Fourth, the drying of concrete containing

18 aqueous solutions (with deicers) differs from that of water. The equilibrium relative

19 humidity of the aqueous solution plays an important role on limiting drying. Finally, the

20 presence of deicing salts in field samples impacts the absorption when field samples are

21 tested in the lab using water. This suggests that care must be taken in analyzing field

22 concrete exposed to deicing salt solutions. 


\section{$\underline{6.1 \text { Overview }}$}

Fluid penetration in concrete has received significant attention from scientists and

5 engineers over the past several decades. To better understand the mechanisms of fluid

6 transport many test configurations have been developed. This chapter will focus on the

7 comparison of the ASTM C-1585 testing procedure with a modified version of the ISAT

8 (Initial Surface Absorption Test) for which the data collection can be automated. A

9 comparison of the two methods is presented.

10 This automated procedure yields results that are similar to the standard ASTM C-

111585 test. The repeatability of the automated procedure is similar or in some cases better

12 than the standard test. Further, the automated test provides additional information that can

13 be used to determine the rate of absorption.

15 The durability of concrete subjected to aggressive environments depends largely

16 on the penetrability of the pore system (Sabir et al. 1998, ASTM 2004). Three

17 mechanisms can be used to describe transport of water in cementitious systems:

18 permeability, diffusion and absorption. Permeability is the measure of the flow of water 
1 under a pressure gradient while diffusion is the movement of ions in a saturated or

2 partially saturated material. Absorption can be described as the materials ability to take in

3 water by means of capillary suction. Water absorption is an important factor for

4 quantifying the durability of cementitious systems (Sabir et al. 1998, Tremblay et al.

5 2005) and is the primary focus in this chapter.

6 The test method currently used to determine the rate of absorption (sorptivity) of

7 water in unsaturated hydraulic cement concretes is ASTM C1585 (2004). This test

8 consists of exposing one surface of a specimen (bottom surface) to water and measuring

9 the increase in mass resulting from absorption. The absorption test involves recording

10 incremental mass change measurements during the first six hours after sample is in

11 contact with water and taking one measurement every day for the next eight days. It is

12 important to note that before the test is conducted, samples are conditioned for 18 days.

13 During the first 3 days, samples are placed in a $50{ }^{\circ} \mathrm{C}$ and $80 \% \mathrm{RH}$ environment. After

14 three days the samples are removed from the chamber and placed in individual sealed

15 container where the samples remain for 15 days to allow internal moisture equilibrium

16 before the test begins.

17 While ASTM C1585 is simple, it can be a time consuming test to conduct. An

18 automated test method which allows for continuous data recording has been

19 implemented. The procedure based on test performed by Tremblay et al. (2005) and

20 Bégué et al. (2004), consists of measuring the decrease of weight of a continuous supply

21 of water connected with the tested specimen, instead of measuring the change in weight

22 of the specimen as in the standard procedure. It should also be noted that the automated

23 test considers both gravity and absorption effects. Previous work by Young (2007) 
1 indicated that capillary effects were similar to the combined effect of gravity and

2 capillary, when there are not cracks or large pores in the sample.

3 The focus of this research is the assessment of a procedure that used automated

4 data collection system for sorption test. The standard procedure (ASTM C1585) and the

5 proposed procedure will be performed on references mortar and concrete cylindrical

6 specimens. By comparing the total amount of absorbed water, and the initial and

7 secondary rate of absorption, the repeatability of both procedures will be evaluated.

\subsection{Standard ASTM C-1585 Procedure}

9 ASTM C-1585 consists of exposing one surface of a specimen (bottom surface) to

10 water and measuring the increase in mass resulting from absorption of the water over

11 time. The mass of the specimen is recorded at 1, 5, 10, 20, 30 and 60 min, then every

12 hour up to $6 \mathrm{~h}$, and then every $24 \mathrm{~h}$ up to $8 \mathrm{~d}$. The amount of absorbed water is

13 normalized by the cross-section area of the specimen exposed to the fluid using Equation

$14 \quad 6.1$ (ASTM, 2004):

$15 \quad \mathrm{I}_{\mathrm{t}}\left(\mathrm{mm}^{3} / \mathrm{mm}^{2}\right)=\frac{\mathrm{m}_{\mathrm{t}}}{(\mathrm{A} \cdot \mathrm{d})}$

16 where " $\mathrm{m}_{\mathrm{t}}$ " is the change in specimen mass in grams at the time $\mathrm{t}$; " $\mathrm{A}$ " is the exposed

17 area of one surface of the specimen, in $\mathrm{mm}^{2}$; and " $\mathrm{d}$ " is the density of the water in $\mathrm{g} / \mathrm{mm}^{3}$

$18\left(0.001 \mathrm{~g} / \mathrm{mm}^{3}\right.$ at $\left.23{ }^{\circ} \mathrm{C}\right)$. 
This normalized value of absorption $\left(\mathrm{I}_{\mathrm{t}}\right)$ is plotted against the square root of time

2 beginning with the addition of water, in order to determine the initial and secondary rate

3 of absorption (ASTM, 2004). The initial rate of water absorption is defined as the slope

4 of the mass of water plotted versus the square root of time considering all the points from

51 minute to 6 hours. The secondary rate of absorption is calculated considering all points

6 from 1 day to 8 days. These periods of time assumes that the fluid that is absorbed by the

7 sample is water. However if other fluids are considered, the period of times considered

8 for initial and secondary rates of absorption may change.

9 ASTM states that the repeatability can be expressed as a coefficient of variation

10 that has been determined to be less than $6.0 \%$ in preliminary measurements for the

11 absorption as measured by this test method for a single laboratory and single operator. It

12 should be noted that if the correlation coefficient is less than 0.98 , the rate of water

13 absorption can not be determined (ASTM 2004).

\subsection{Proposed Procedure}

15 This procedure consists of measuring the decrease of weight of a continuous 16 supply of water connected with the top surface of the tested specimen, instead of

17 measuring the change in mass of the specimen as in the standard procedure. The set-up 18 and procedure proposed is based on test performed Tremblay et al. (2005) and Bégué et 19 al. (2004). Figure 6.1a shows a photo of two samples being tested using the automated 20 procedure. 


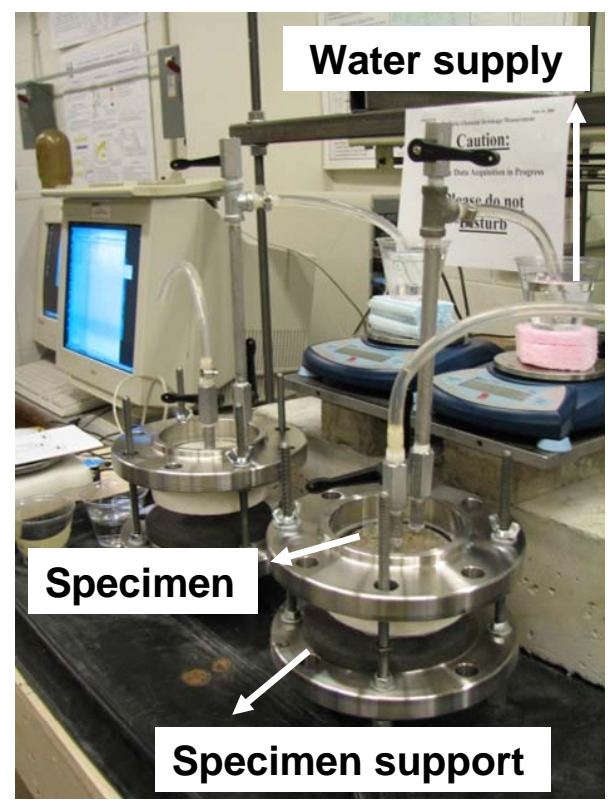

a)

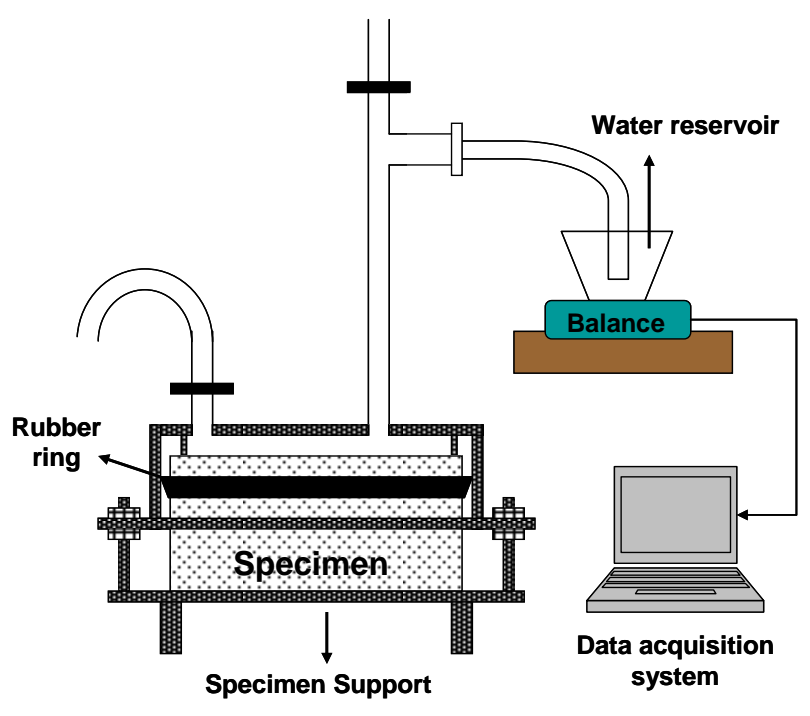

b)
4 of the setup during the test (Figure 6.1). The edge between the rubber ring and the 5 specimen support is sealed by contact at the surfaces.

6 The test starts (i.e., time $=$ zero) when water is introduced in the system using the

7 pipe 1 (Figure 6.2a). The air inside of the system is removed using the pipe 2 . When air is 8 completely removed, the stopcock \#2 is closed (Figure 6.2b). Additional water is

9 introduced using pipe 1. When the water level rises to be over stopcock \#1, it is closed.

10 Then more water is added using pipe 3 until it is complete filled with water.

11 Subsequently, pipe 3 is turn down using a finger over the end of it to avoid the loss of

12 water from the pipe. The pipe is then introduced in the water reservoir and it is placed on

13 a digital balance connected to a computer with an automated data collection system 
1 (Figure 6.2c). Paraffin oil is added carefully on the top of the water reservoir to minimize

2 evaporation. Finally, the automated data collection system is activated recording mass 3 and time.

4 The time elapsed between the moment that water is in contact with the specimen 5 and the moment that the data collection system is approximately 4 to 5 minutes. The 6 initial rate and secondary rate of absorption will be not affected if the absorption during 7 the initial 5 minutes is not known. However the total absorbed mass will change. In order 8 to know the total absorbed water, the specimen weight is recorded before and after the 9 test. Comparing this change of mass with the data recorded by the automatic collection 10 system, is possible to determine the absorption during the initial minutes. 


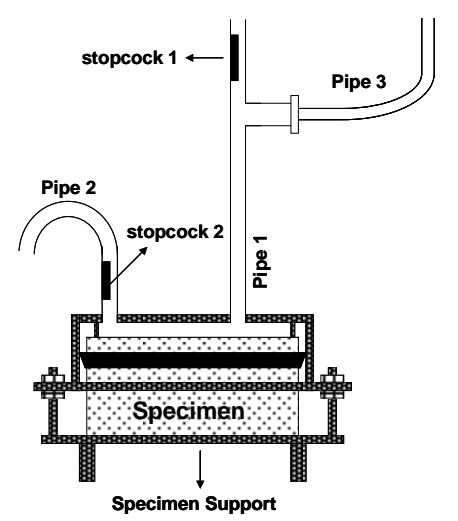

a) Step 1: Introduce specimen

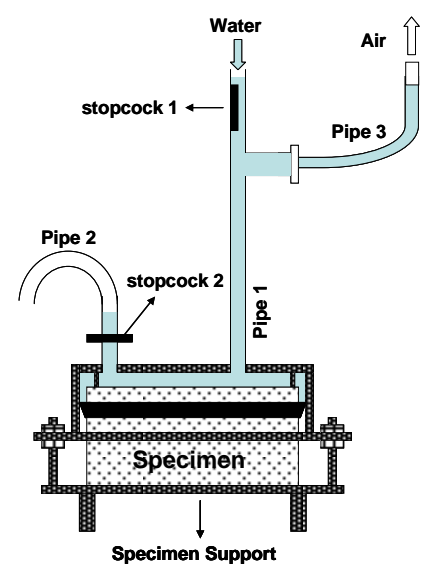

c) Step 3: Close stopcock 2, fill pipe 1

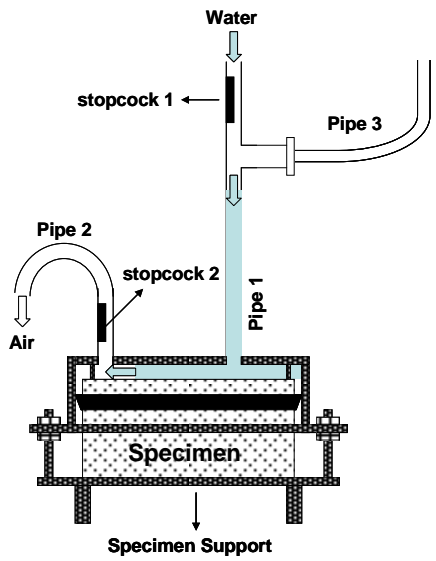

b) Step 2: Water in, Air out

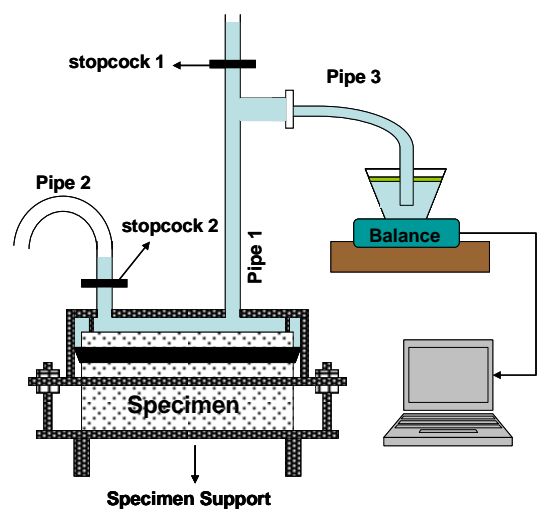

d) Step 4: Close stopcock 1, fill pipe 3, connect pipe 3 with water supply

3 were performed. The first test has for objective to measure the lost of mass from the

4 water reservoir because of evaporation. Three different water reservoirs were filled with

5 approximately 150 grams of water and covered with 30 grams of paraffin oil. Then, they

6 were placed in an environment similar to the one where the actual sorption tests are 
1 performed $\left(23 \pm 2{ }^{\circ} \mathrm{C}\right.$ and $50 \pm 3 \% \mathrm{RH}$ environment). The change of mass was monitored

2 during 8 days. The results show that the average evaporation from the water reservoir is

30.15 grams over 8 days with a low variability (Figure 6.3).

4 The second test consisted of performing a regular test, but a plastic (impermeable)

5 sample was used instead a real concrete sample with the objective to simulate a test on a

6 low permeability material. The results of these tests, that include the effect of evaporation

7 from the source of water, show that the setup is not completely impermeable, but it is

8 controllable under the environment in the laboratory (Figure 6.3). Total measured

9 evaporation was $0.4 \mathrm{~g}$ of water, while the total change in mass from a sorption test is

10 approximately $25 \mathrm{~g}$. This would result in an error of less than $2 \%$ generated by the loss of

11 water from the reservoir of water covered with paraffin oil.

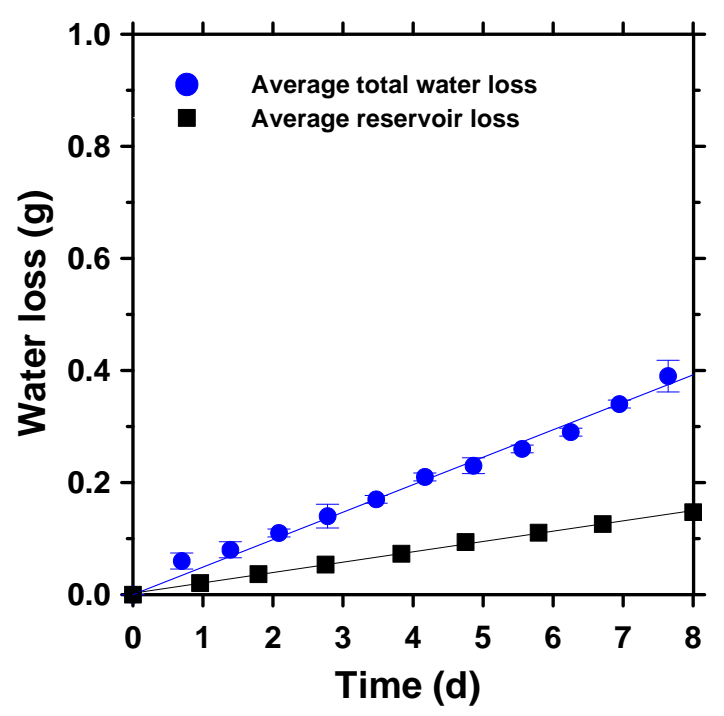

13 Figure 6.3: Total Water Loss During a Test of an Impermeable Plastic Sample and Total 14 Evaporated Water from Reservoir of Water Covered with Paraffin Oil 
1 Once evaporation value is known, it is necessary considering it to correct

2 measurements on actual samples. According with the experimental results, 0.4 gram of

3 water will be subtracted from the total amount of absorbed water in a regular test of 8

4 days. Assuming linear behavior of total evaporated water will simplify the procedure and

5 it will not affect significantly the results (Figure 6.3).

6

11 C-40 with the number on the left representing $w / c$. A list of the mixture proportions can

12 be found in Table 6.1

\subsection{Experiment Work}

For this study four different mixtures were prepared in total. Three mixtures were mortars with a single volume fraction of fine aggregate $(55 \%)$ and different $w / c(w / c$ of $0.40,0.45$ and 0.50$)$. These mixtures were designated as M-40, M-45 and M-50, with the numbers representing the different $w / c$. The fourth mixture was concrete, designated as

be found in Table 6.1

Table 6.1: Mixture Proportions, SSD Condition

\begin{tabular}{lcccc}
\hline Constituents & $\mathrm{M}-40$ & $\mathrm{M}-45$ & $\mathrm{M}-50$ & $\mathrm{C}-40$ \\
\hline Cement type I $\left(\mathrm{kg} / \mathrm{m}^{3}\right)$ & 625.7 & 584.9 & 549.2 & 316.0 \\
Pozzolan & 0 & 0 & 0 & 60.0 \\
Water $\left(\mathrm{kg} / \mathrm{m}^{3}\right)$ & 250.8 & 263.7 & 275.1 & 150.4 \\
Fine aggregate $\left(\mathrm{kg} / \mathrm{m}^{3}\right)$ & 1389.6 & 1389.6 & 1389.6 & 736.3 \\
Coarse aggregate $\left(\mathrm{kg} / \mathrm{m}^{3}\right)$ & 0 & 0 & 0 & 1049.2 \\
\hline w/c or w/b & 0.40 & 0.45 & 0.50 & 0.40 \\
\hline Aggregate volume & $55 \%$ & $55 \%$ & $55 \%$ & $60 \%$ \\
\hline
\end{tabular}

14 Cylinders (101.6 $\mathrm{mm}$ or 4 inches and $203.2 \mathrm{~mm}$ or 8 inches diameter) were cast

15 for each mixture. The specimens were cured for 28 days in double sealed bags at 23 
$1 \pm 1^{\circ} \mathrm{C}$. After curing, three $50.8 \mathrm{~mm}(2$ inches) slices thick were extracted from central

2 portion of each cylinder. The remaining outer portions were discarded.

3 Slices were conditioned in three different environmental chambers at three

4 different relative humidities, $50 \pm 2 \%, 65 \pm 2 \%$ and $80 \pm 2 \%$ until they reached mass

5 equilibrium. The temperature in all three chambers was fixed at $23 \pm 1{ }^{\circ} \mathrm{C}$. After

6 specimens reached mass equilibrium (approximately 12 months), the mass and

7 dimensions of each sample was recorded. The outer edge of each slice was coated with a

8 thin film of epoxy to prevent the moisture exchange from the lateral surface during test.

9 Additionally, the specimens to be use in the proposed procedure were coated in

10 the bottom surface with epoxy to avoid evaporation during the test. If the bottom surface

11 is not sealed, unquantifiable amount water will be evaporated through that surface

12 affecting the measured absorption. A rubber ring is placed on the specimens to avoid

13 leaking between the specimen and the specimen's support during the test. The edge

14 between the sample and the ring is sealed using caulking silicone (Figure 6.4). When the

15 silicone is hardened, the total mass specimen with epoxy and ring is recorded. 


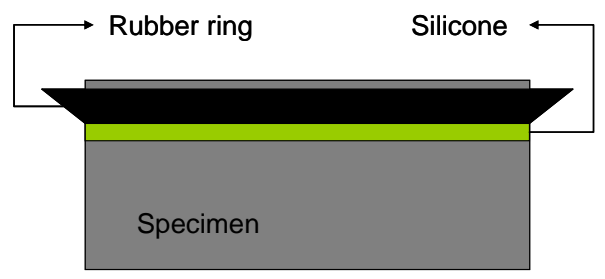

(a)

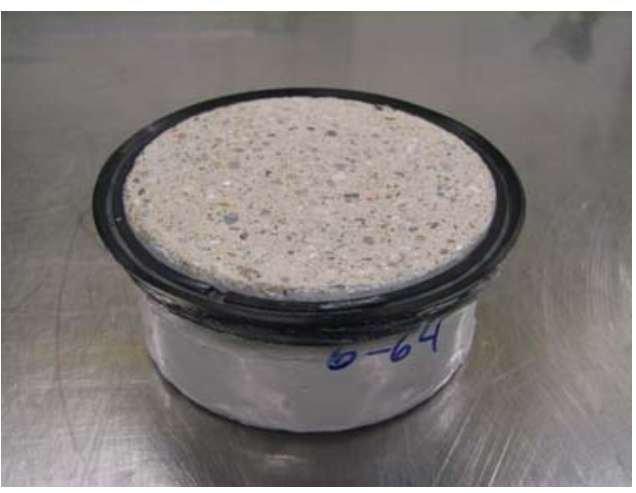

(b)

$1 \quad$ Figure 6.4: Preparation of Specimen for Automates Procedure a) Representation of Sample Preparation b) Photo of the Sample Ready to be Tested

\section{$\underline{6.6 \text { Results }}$} The standard and the automated procedures were performed on the specimens.

5 Four samples from each specimen were tested (two for each procedure). Figure 6.5 and

66.6 shows a comparison between the average results of absorption of the standard and the

7 proposed procedure for the four different specimens. In the plots, the dashed lines 8 represent the results from the automated procedure. 

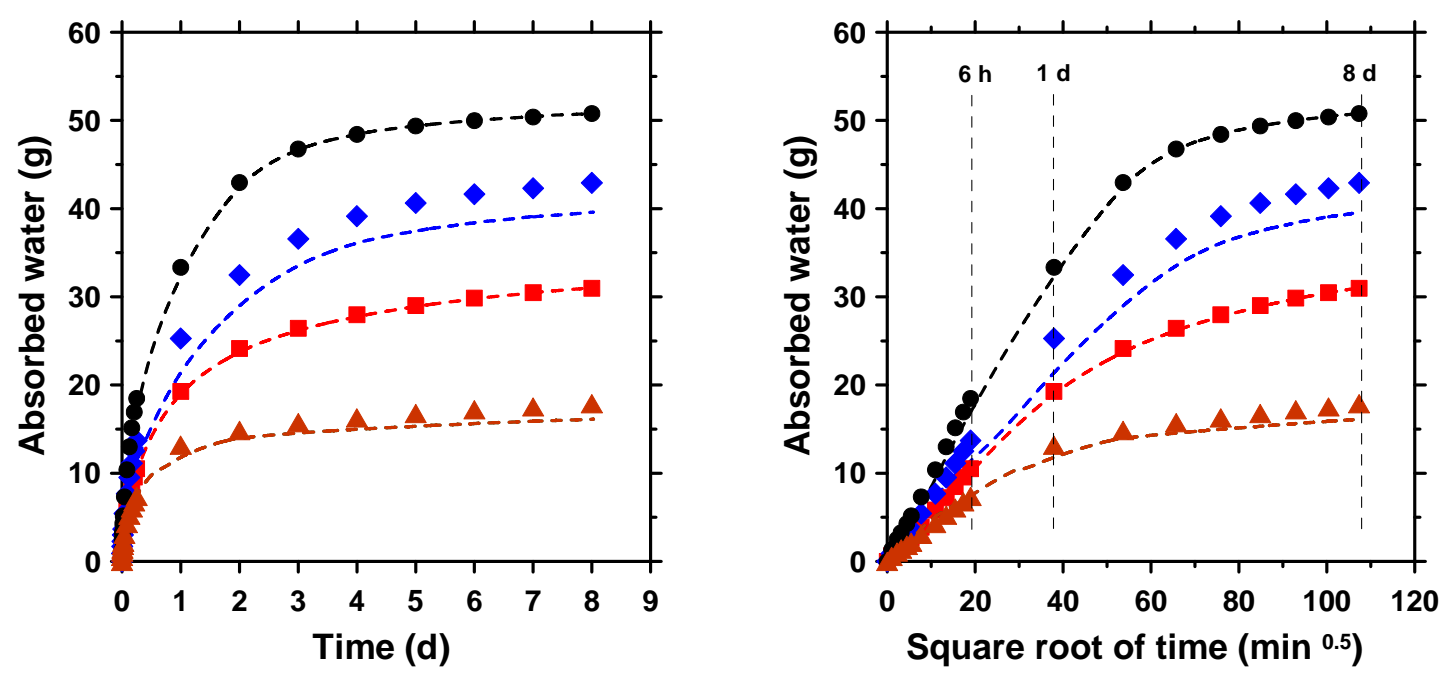

a)

b)

3 Figure 6.5: Comparison of Absorbed Water of Samples Conditioned at 50\%RH: a) vs time, b) vs Square Root of Time
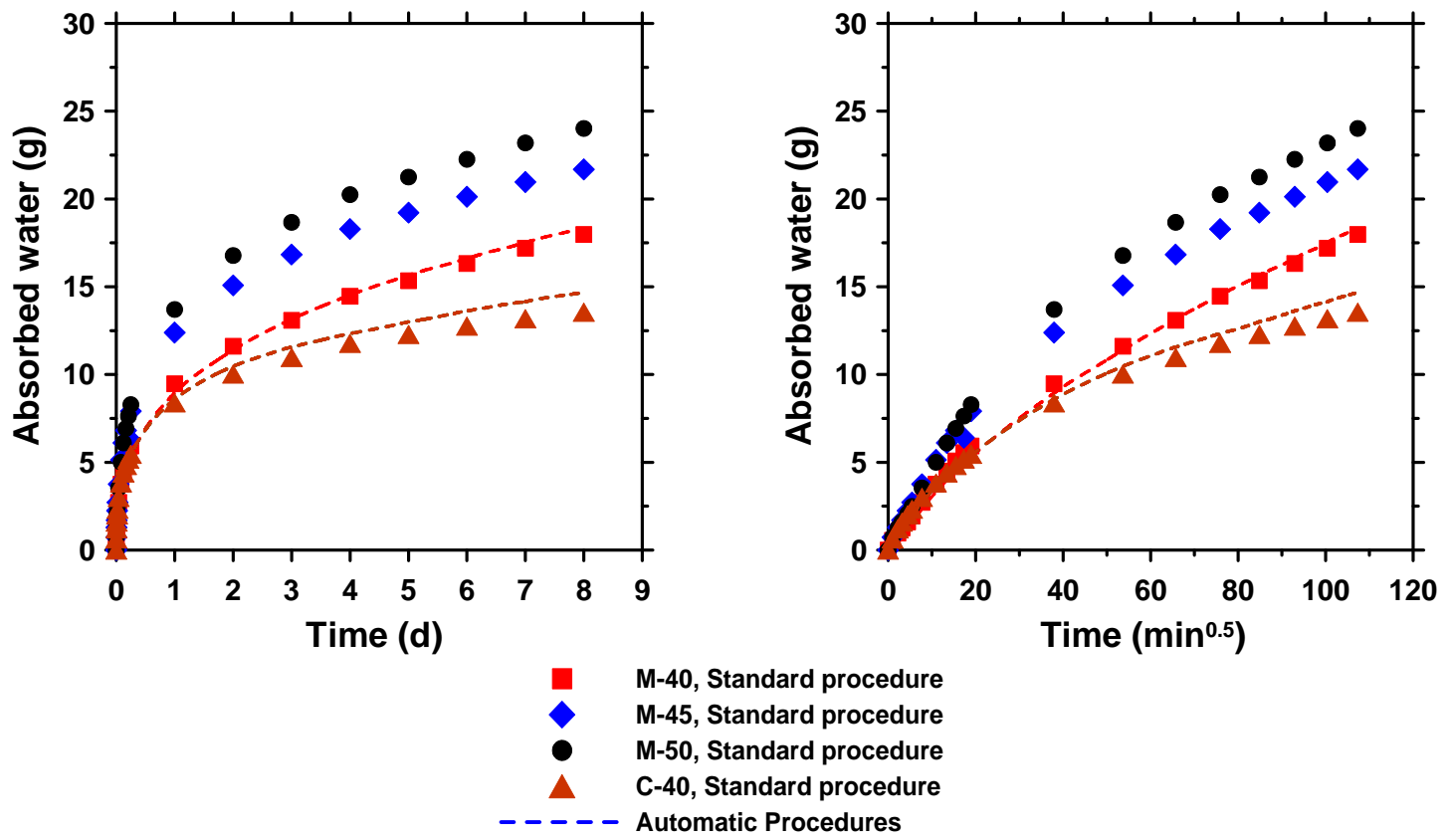

a)

b)

7 Figure 6.6: Comparison of Absorbed Water of Samples Conditioned at 65\%RH: a) vs. 


\section{$\underline{6.7 \text { Summary and Conclusions }}$}

2 ASTM C1585 is currently used to determine the rate of water absorption by

3 hydraulic cement concretes. ASTM C1585 consists of exposing one surface of a 4 specimen to water and measuring the increase in mass due to water absorption. While

5 this test is easy to perform, it can be time consuming and can require the timing of data

6 collection to be altered if fluids other than water are used.

7 A procedure is used here, which allows for continuous and automated data

8 acquisition. This automated method consists of measuring the decrease in mass of a

9 continuous supply of water (or other fluid) connected with the tested specimen, instead of

10 measuring the change in mass of the specimen directly. The supply of water is placed on

11 a digital balance and the balance is connected to a computer with an automated data

12 collection system. This enables data to be collected at a much more frequent rate.

13 Both the standard and automated procedures were performed on two different

14 specimens. The proposed procedure yields reproducible values of rates of absorption that

15 are similar to those of the ASTM standard test.

16 In addition, the proposed procedure decreases the influence of the operator, and is

17 less time consuming after the test started. This additional data can provide information

18 for further analysis of the absorption behavior of the samples. 
5 concrete. The electrical conductivity, or resistivity, of concrete is frequently used as a

6 surrogate test method for assessing a concrete's fluid transport properties. This work

7 indicates benefits of using the continuous monitoring of electrical conductivity from the

8 time of casting and also discusses rapid conductivity measurements. Both of these

9 methodologies could provide a valuable alternative to rapid chloride permeability (RCPT,

10 ASTM C1202). This work highlights the need to carefully consider the effects of

11 temperature, hydration, sample geometry, and pore solution concentration when

12 interpreting the electrical measurements using the modified parallel model. The work

13 describes the electrical conductivity measurements from a series of concretes that are cast

14 with different water to cement ratios, aggregate volumes, and admixtures.

17 electrical impedance of a concrete by applying a difference in potential across the 18 concrete (Rajabipour 2006). The resulting current is measured, and an impedance and 
1 phase base can be determined. The impedance can be broken into two parts: real and

2 imaginary. By measuring the electrical impedance of concrete over time, information can

3 be obtained about the fluid transport properties that will influence service life predictions

4 for concrete structures (McCarter and Curran 1984, Christensen et al. 1994, Ford and 5 Mason 1996).

6 The EIS described in this paper is performed by applying an alternating current 7 (AC) to the concrete. AC is preferred as compared to direct current (DC) for a few 8 reasons. First, DC causes the ions in the concrete to become "polarized" meaning certain 9 ions become preferentially attracted to one electrode depending on their charge (Bockris 10 and Reddy 1970, McCarter and Curran 1984, McCarter and Brousseau 1990, Gu and 11 Beeaudoin 1996). This crowding of ions around an electrode makes it hard for some 12 oppositely charged ions to migrate through the system, yielding spurious results. Second, 13 the voltage used for DC measurements is typically much greater than that of AC 14 measurements. This increased voltage means increased power needed to perform the test.

15 The increased power in the sample can input a large amount of energy into the system 16 causing an increase in the temperature or this energy can result in damage to the 17 microstructure of the concrete. The primary mode of conduction in concrete is through 18 the pore solution. Since the electrical properties of the liquid phase of a concrete (the 19 pore solution) is dependent on temperature, a change in the temperature of the sample can 20 give inconsistent results if this is not accounted for.

21 As stated previously, EIS measures the resistance of a material to an applied

22 current. In EIS the resistance of this current is measured at multiple frequencies. Two 23 graphs are frequently used to assess data from EIS measurements: the Nyquist plot and 
1 the Bode plot. The Nyquist plot places the real impedance (commonly thought of as

2 resistance) on the ordinate axis and the corresponding imaginary impedance (commonly

3 thought of as capacitance) on the abscissa. The bulk resistance of the material is

4 determined when the imaginary component is at a minimum. The advantage of the

5 Nyquist plot is that the resistance value can be easily determined through visual

6 recognition. The Bode plot contains the frequency of the electrical current on the

7 ordinate and the impedance on the abscissa. The influence of frequency is more easily

8 realized on the typical Bode plot (Figure 7.1).

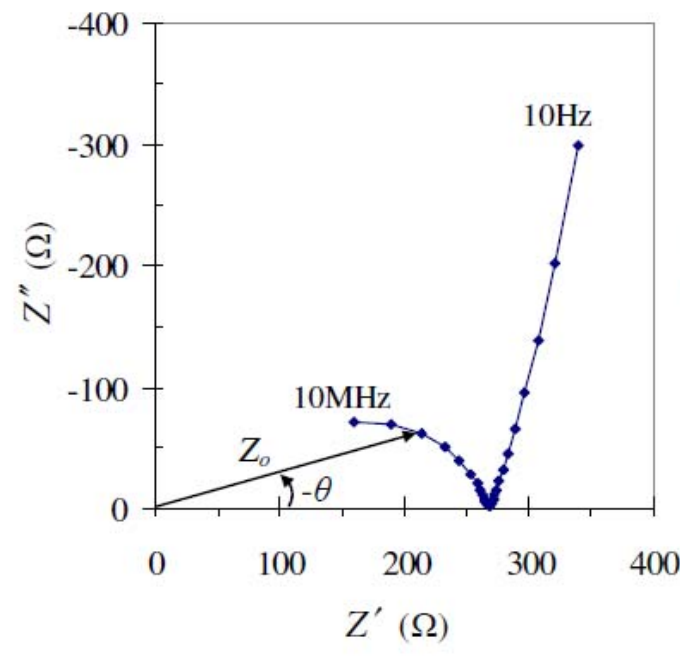

9

Figure 7.1: Nyquist Plot and Bode Plot (after Macdonald and William 1987)

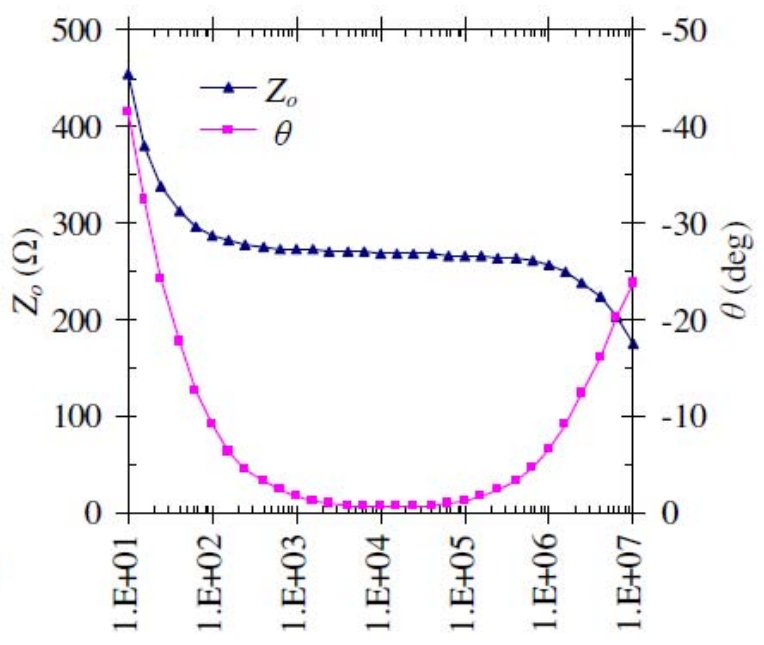

Freq $(\mathrm{Hz})$ 


\section{$\underline{7.3 \text { Objectives }}$}

The objective of this work was four-fold:

- First, the chapter outlines a procedure and sample geometry to collect field samples for possible Quality Control / Quality Assurance (QA/QC) practices. The EIS procedure will calculate similar properties yielded by the RCPT, but with more repeatable results, and more early age data.

- Second, the chapter reports experimental results of electrical impedance, temperature rise, degree of hydration, and pore solution conductivity as a function of time. The results indicate the effect of correcting conductivity measurements.

- Third, the chapter provides a systematic study of the effects of temperature on electrical conduction. This is essential to enable electrical measurements to be corrected for temperature.

- Fourth, a sensor will be evaluated that assesses changes in pore solution as a function of hydration.

\subsection{Experimental Program}

\subsubsection{Program Overview}

Nine different mixture designs were evaluated. After the samples were cast, they were monitored for the following week continuously. After the continuous first week, the samples were point measured once a week for the next three weeks. Finally, after the 
1 first four weeks, the samples were point measured once every four weeks till an age of 90

2 days was reached. This is explained in Table 7.1. The program itself can be broken

3 down into four main categories: equal paste comparison (Table 7.2), equal w/c

4 comparison (Table 7.3), increased water content comparison (Table 7.4), and admixture

5 comparison (Table 7.5).

6

7

Table 7.2: Equal Paste Content Testing Breakdown

9
Table 7.1: Testing Schedule

\begin{tabular}{|c|c|}
\hline Age (weeks) & Tested \\
\hline $0-1$ & Continuously \\
\hline $2-4$ & Weekly \\
\hline $5-12$ & Every 4 weeks \\
\hline
\end{tabular}

\begin{tabular}{|c|c|c|}
\hline \multicolumn{3}{|c|}{ Equal Paste Content Samples } \\
\hline Mixture \# & w/c & $\begin{array}{c}\text { Paste Content } \\
\text { (\% volume) }\end{array}$ \\
\hline 1 & 0.36 & 27.7 \\
\hline 2 & 0.42 & 27.7 \\
\hline 3 & 0.45 & 27.7 \\
\hline 4 & 0.50 & 27.7 \\
\hline
\end{tabular}


Table 7.3: Equal Water to Cement Ratio Testing Breakdown

\begin{tabular}{|c|c|c|}
\hline \multicolumn{3}{|c|}{ Equal Paste Content Samples } \\
\hline Mixture \# & w/c & $\begin{array}{c}\text { Paste Content } \\
\text { (\% volume) }\end{array}$ \\
\hline 2 & 0.42 & 27.7 \\
\hline 5 & 0.42 & 34.0 \\
\hline
\end{tabular}

Table 7.4: Increased Water Content Testing Breakdown

\begin{tabular}{|c|c|c|c|c|}
\hline \multicolumn{5}{|c|}{ Increased Water Content } \\
\hline Mixture \# & Original w/c & $\begin{array}{c}\mathrm{kg} \text { of water } \\
\text { added per } \mathrm{m}^{3}\end{array}$ & Final w/c & $\begin{array}{c}\text { Paste Content } \\
\text { (\% volume })\end{array}$ \\
\hline 2 & 0.42 & 0 & 0.42 & 27.7 \\
\hline 6 & 0.42 & 10.9 & 0.45 & 28.4 \\
\hline 7 & 0.42 & 29.1 & 0.50 & 29.7 \\
\hline
\end{tabular}

Table 7.5: Increased Admixture Testing Breakdown

\begin{tabular}{|c|c|c|c|c|}
\hline \multicolumn{5}{|c|}{ Admixture Addition } \\
\hline Mixture \# & w/c & Used additive & $\begin{array}{c}\text { g admixture per 100 } \\
\text { g of cement }\end{array}$ & $\begin{array}{c}\text { Paste Content (\% } \\
\text { volume) }\end{array}$ \\
\hline 2 & 0.42 & none & - & 27.7 \\
\hline 8 & 0.42 & air entrainer & 0.05 & 27.7 \\
\hline 9 & 0.42 & water reducer & 0.20 & 27.7 \\
\hline
\end{tabular}

9 practice. These practices are usually related to strength of the concrete. Additional

10 information is needed to find the properties that can be used to predict the service life of

11 the concrete. An early age prediction of fluid transport properties would give DOT's 
1 another criteria for acceptance of a concrete and overall better prediction of service life.

2 The specimen geometry used in this research was developed with a possible QC/QA

3 procedure in mind. A standard 6"x12" cylinder mold was outfitted with a pair of

4 electrodes used to conduct the EIS experiments. The use of a 12" cylinder mold should

5 make any future $\mathrm{QA} / \mathrm{QC}$ development a simple transition and is compatible with any

6 storage equipment designed to accommodate a standard cylinder. A detailed description

7 can be found in section 7.4.5.

8 The specimens were monitored continuously for one week after casting, weekly

9 from 2-4 weeks of age, and monthly from 2 months-90 days. The change in the

10 conductivity of a 3 day old concrete sample is very small. Because of this, the testing

11 regimen utilized provides a representative amount of data over the desired time scale.

\subsubsection{Mixture Proportions}

13 Four different w/c $(0.36,0.42,0.45$ and 0.50$)$ and four different paste volume

14 fractions $(27.7 \%, 28.4 \%, 29.7$ and $34.0 \%$ of the total volume) were used. These mixtures

15 were designated as $27.7 / 0.42,27.7 / 0.45,27.7 / 0.50,34.0 / 0.42,28.4 / 0.42$ and $29.7 / 0.42$

16 with the number on the left representing the volume fraction of cement paste and the

17 number on the right representing $w / c$. Mixtures 8 and 9 are have the same mixtures that

18 mixture 2, but adding air entrainer and water reducer respectively, as shown in Table 7.5.

19 A list of the mixture proportions can be found in Table 7.6. 
Table 7.6: Mixture Proportion for Electrical Conductivity Testing, in SSD Condition

\begin{tabular}{|l|r|r|r|r|r|r|r|}
\cline { 2 - 8 } \multicolumn{1}{c|}{} & \multicolumn{7}{c|}{ Mixtures } \\
\hline \multicolumn{1}{c|}{ Constituent } & $\mathbf{2 7 . 7 / 0 . 3 6}$ & $\mathbf{2 7 . 7 / 0 . 4 2}$ & $\mathbf{2 7 . 7 / 0 . 4 5}$ & $\mathbf{2 7 . 7 / 0 . 5 0}$ & $\mathbf{3 4 . 0 / 0 . 4 2}$ & $\mathbf{2 8 . 4 / 0 . 4 5}$ & $\mathbf{2 9 . 7 / 0 . 5 0}$ \\
\hline Coarse Aggregate, $\mathrm{IN} \# 8\left(\mathrm{~kg} / \mathrm{m}^{3}\right)$ & 1047.2 & 1047.2 & 1047.2 & 1047.2 & 944.6 & 1035.9 & 1017.6 \\
\hline Fine Aggregate, $\mathrm{IN} \# 23\left(\mathrm{~kg} / \mathrm{m}^{3}\right)$ & 880.4 & 880.4 & 880.4 & 880.4 & 786.6 & 870.9 & 855.5 \\
\hline Cement Type $1(\mathrm{~kg} / \mathrm{m} 3)$ & 396.4 & 364.2 & 350.2 & 328.7 & 464.5 & 360.3 & 353.9 \\
\hline Water, Public Source $(\mathrm{kg} / \mathrm{m} 3)$ & 142.7 & 152.9 & 157.5 & 164.3 & 195.4 & 162.1 & 176.9 \\
\hline Admixtures $(\mathrm{g} / 100 \mathrm{~g}$ of cement) & 0.0 & 0.0 & 0.0 & 0.0 & 0.0 & 0.0 & 0.0 \\
\hline
\end{tabular}

$4 \quad$ 7.4.4.1.Portland Cement

An ASTM C150 Type I ordinary portland cement (OPC) was used in this study,

6 with a Blaine fineness of $370 \mathrm{~m}^{2} / \mathrm{kg}$ and an estimated Bogue composition of $56 \% \mathrm{C}_{3} \mathrm{~S}$,

$7 \quad 16 \% \mathrm{C}_{2} \mathrm{~S}, 12 \% \mathrm{C}_{3} \mathrm{~A}, 7 \% \mathrm{C}_{4} \mathrm{AF}$ and a $\mathrm{Na}_{2} \mathrm{O}$ equivalent of $0.68 \%$ by mass.

9 The sand used was natural river sand with a fineness modulus of 2.71, an apparent

10 specific gravity of 2.58 , and an absorption of $1.8 \%$ by mass.

\section{$11 \quad$ 7.4.4.3 Coarse Aggregate}

12 The coarse aggregate used was limestone with an apparent specific gravity of 132.65 , and an absorption of $0.9 \%$ by mass. 


\subsubsection{Specimen Geometry and Casting}

2 The two major experiments in the research required two different sample

3 geometries. Both the EIS experiment and the late age pore solution extraction geometries 4 are detailed below.

\section{$5 \quad$ 7.4.5.1 EIS Concrete Samples}

6 The mold preparation starts with a 6" diameter, 3/8" thick, high density poly7 ethylene (HDPE) disk (Figure 7.2). The disk is cut using a hole saw with a 6" inner

8 diameter from a larger sheet of HDPE. Then, two holes are drilled four inches, on center, 9 away from each other. These holes are then tapped using a 3/8"-16 tap. Inside these

10 holes, 12" threaded stainless steel rods are screwed until the tip of the rod is flush with

11 one end of the HDPE disk.

12 It is at this time some type of 1" thick spacer material is placed at the bottom of a

13 standard 6"x12" concrete cylinder mold. In the current experiment, a 4.25" square of

14 extruded polystyrene insulation was used. Then, inside the cylinder mold, the disk with

15 the stainless steel rods threaded to it is placed, disk side down, until the disk is flush with

16 the spacer material (Figure 7.2). The rods should stick out of the top of the cylinder by

17 about an inch to accommodate the EIS equipment connection. A schematic representation

18 of the mold is shown in Figure 7.3. 


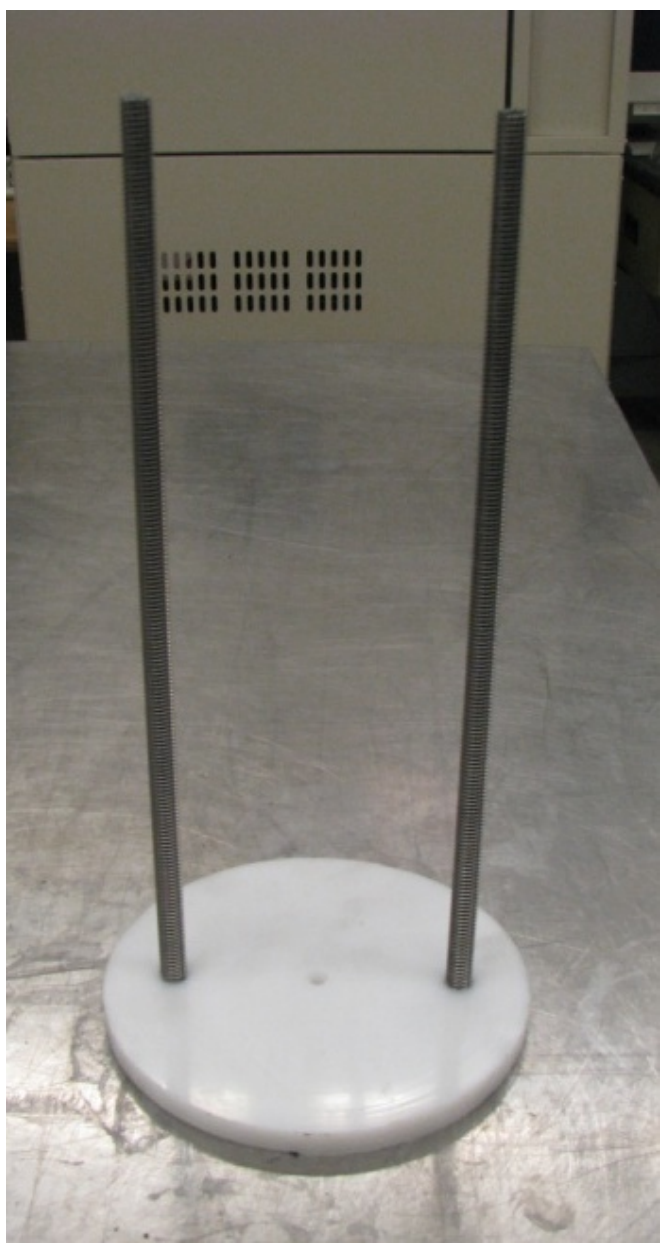

Figure 7.2: HDPE Disk and Threaded Rod Apparatus 
4 volume of the mold with each lift. The lifts are roded twenty five times each, paying

5 special attention to the area around the stainless steel rods. After each lift, a vibrating

6 table is used to vibrate the sample for 5-10 seconds. The top is finished with a trowel.

7 After the mold is full of material, a standard 6"x12" cylinder mold lid with two 3/8"

8 holes spaced 4 inches apart, on center, is placed on top (Figure 7.4). After the lid is

9 secure, the sample is again vibrated for 3-5 more seconds while being tapped with an

10 open hand around the outside. After the lid is place on top of the mold, be sure to wipe

11 off the threads of the portion of the rods that are sticking out of the mold so that screws 
1 can be threaded on to the rods and good connection can be made when the rods act as

2 terminals later.

3 Within one hour from the initial mixing, a thermocouple is placed in the cylinder

4 to monitor the temperature, and the electrodes are attached to the EIS analyzer to acquire 5 the impedance data.

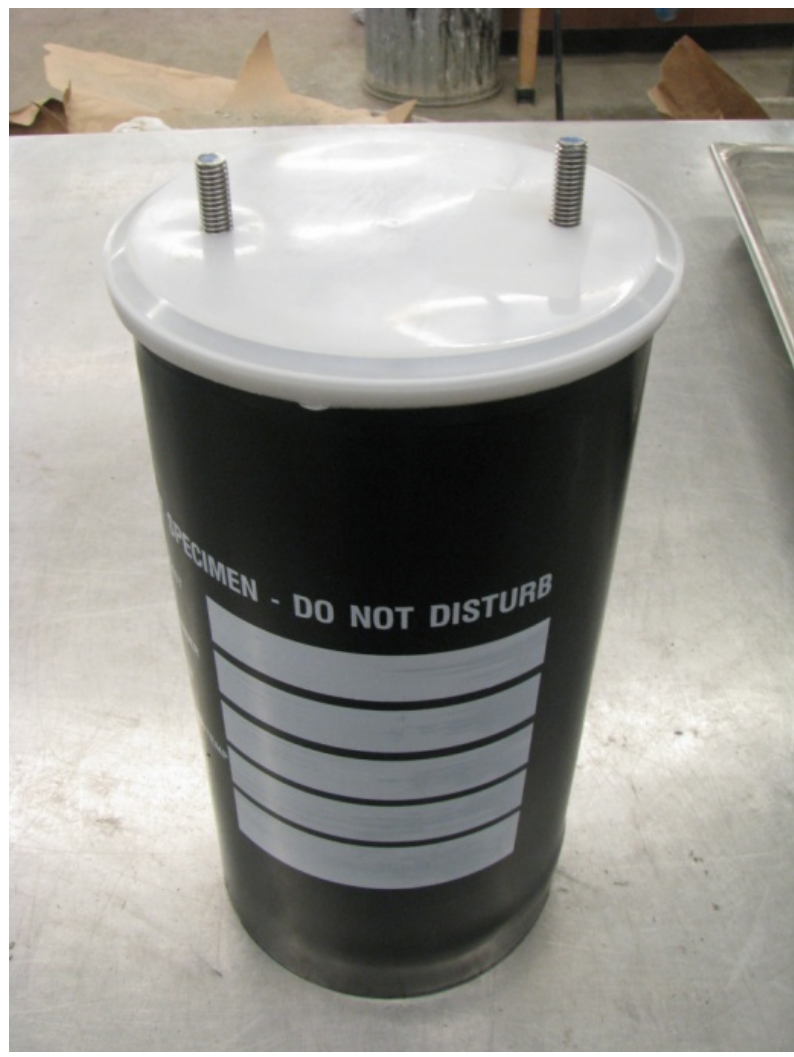

Figure 7.4: Finished Specimen with Rods and Lid in Place 
7 increasing ion concentration as the cement continues to hydrate and react with the water,

8 reducing the amount of pore solution. To characterize this increase in conductivity, the

9 pore solution must be extracted from the other media in order to determine its overall

10 contribution to the parallel model discussed earlier. The pore solution is extracted

11 through two different processes; one used for samples before set and the other for after 12 set has occurred.

13 Samples with four different water to cement ratios were created: $0.36,0.42,0.45$,

14 and 0.50 mirroring all the different $\mathrm{w} / \mathrm{c}$ tested in the EIS cylinder experiments. The early

15 age pore solution extractions are taken when the concrete is still in its plastic state, 16 according to the procedure by Penko (1983). Mortar samples are created and the

17 extractions are taken at predetermined times. The same mixtures proportion from Table

18 7-6 are used, but the coarse aggregate is not added in these mixtures. In general, the pore

19 solution extractions were taken at an age of 10 minutes and 1 hour. More pore solution

20 extractions were taken close to set time usually around 3-4 hours, and every hour

21 following until the concrete became to difficult to work with. The lower w/c mixtures

22 become harder to work with more quickly than the higher w/c mixtures, yielding less data 
1 overall. The pore solution samples are kept in air tight vials to protect against

2 carbonation until they undergo conductivity testing.

3 The later age pore solutions were extracted from pore solutions at 12 hour, 20

4 hour, 1 day, 3 day, and 8 day ages. The pore solution samples were obtained by a high

5 pressure piston and cylinder apparatus according to the procedure described by

6 Barneyback (1983). The test consists of placing the sample in a high strength steel

7 cylinder and crushing the sample with a high pressure piston, usually provided by a

8 standard compression testing machine. After the pore fluid is freed from the sample, the

9 fluid is extracted via a syringe. The samples used in this test were pastes only. Paste is

10 preferred when performing piston and cylinder extraction because of the higher yield of

11 pore solution per sample, reducing the time needed to obtain the minimum amount of

12 pore solution, and the lower chance of damaging the testing apparatus. Mortars can cause

13 scoring along the cylinder walls, leading to costly repairs and apparatus downtime.

\section{$\underline{7.5 \text { Experimental Techniques }}$}

16 The concrete sample was attached to a Solartron SI 1260 Impedance/Gain-Phase

17 Analyzer by the two stainless steel rods which act as electrodes. Copper wire is run from

18 the impedance analyzer to each terminal and secured by two stainless steel nuts where the

19 wire is place in between the two nuts and tightened to ensure good connection. The 
1 samples were monitored for a week continuously, once a week thereafter for twenty eight 2 days, and every four weeks until ninety days.

3 The data acquired by the EIS experiment is in the form of a resistance,

4 specifically, the units of ohms. Hereafter, all EIS experimental data will be expressed in

5 the form of conductivity, specifically in the unit of Siemens/meter. To go from resistance

6 value to conductivity value, Equation 7.1 can be used.

$$
\sigma=k / R_{B}
$$

Where:

8

$\sigma=$ The conductivity $(\mathrm{S} / \mathrm{m})$

$9 \quad k=$ The geometry factor $(1 / \mathrm{m})$

$10 \quad R_{g}=$ The bulk resistance $(\Omega)$

11 The geometry factor can be determined by using a solution of known

12 conductivity. This solution is placed in the mold just as the concrete itself is placed in the

13 mold during casting. The resistance is obtained through EIS. If the conductivity of the

14 solution is known, a geometry factor can then be back calculated using Equation 7.1. 


\subsubsection{Automation of Measurements}

2 EIS measurements can be used to monitor the concrete structure as it hydrates.

3 Poursaee and Weiss (2010) developed a user interface using LabView to allow

4 researchers to monitor multiple specimens simultaneously. By automating the process,

5 the experiment has become more much efficient in terms of man hours needed to perform

6 it, and yields better results by automatically acquiring data when it would highly

7 inconvenient to do so in person. Automation is also employed in the data analysis

8 portion of the testing. A program written in visual basic language scans the EIS data file

9 for resistance and relays that value along with the time of the measurement to a

10 spreadsheet file which can be sorted and further analyzed. With both the data acquisition

11 and data analysis portions of the experiment automated, the only part of the test that is

12 time intensive is the sample preparation.

\subsubsection{Pore Solution Conductivity Testing}

14 After the pore solution is extracted from the samples, the solution is tested for 15 conductivity. To test the conductivity of the solution, the fluid is placed in a conductivity 16 cell. The conductivity cell consists of two parallel plates placed on either side of a small

17 plastic cylinder, and can be seen in Figure 7.5. The plates are kept in place using a clamp

18 to apply pressure to both ends. The fluid is injected by a syringe into the cell using a pair

19 of holes drilled on into the wall of the cylinder. From there the cell has a current applied

20 to it using the EIS equipment and the resistance of the bulk material is found. The

21 resistance is converted to a conductivity value. A geometry factor was calculated exactly

22 the same as the EIS sample, by using a fluid of known conductivity, obtaining the 
1 resistance of the fluid in the cell through EIS testing, and back calculating a geometry

2 factor using Equation 7.1. A typical plot of pore solution conductivity versus time can be

3 seen later in Figure 7.9.

4

7 7.6. The conductivity is high initially for the fresh concrete. There is decrease in the

8 conductivity of nearly $90 \%$ during the first few days for this specific mixture design.

9 The high level of repeatability in the measurements can be seen by examining the error 10 bars in Figure 7.6.

A sample of the raw conductivity data from the concrete can be seen in Figure

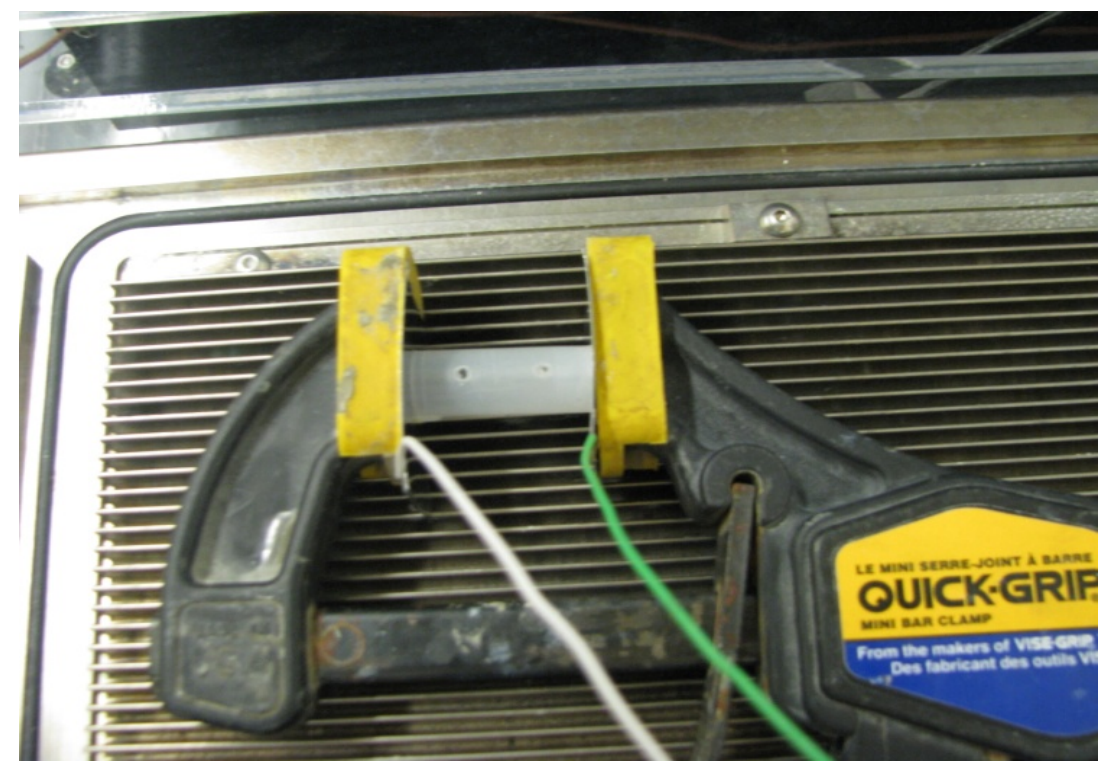

Figure 7.5: Pore Solution Conductivity Cell 
6 states that the conductivity of concrete can be attributed to three components: the

7 conductivity of the pore solution as it is the primary conductive phase in concrete, the

8 volume fraction of pore solution, and the connectivity of the system which reflects how

9 well an ion can pass from one place to another within the system. The model is

10 expressed in equation form in Equation 7.2.

Figure 7.6: Geometry Corrected EIS Conductivity Data

\subsection{Review of the Modified Parallel Law}

Concrete has been modeled by using the modified parallel law (Dullien 1979, McLachlan et al. 1990, Gu et al. 1992, Ford et al. 1995, Torquato 2002). The model

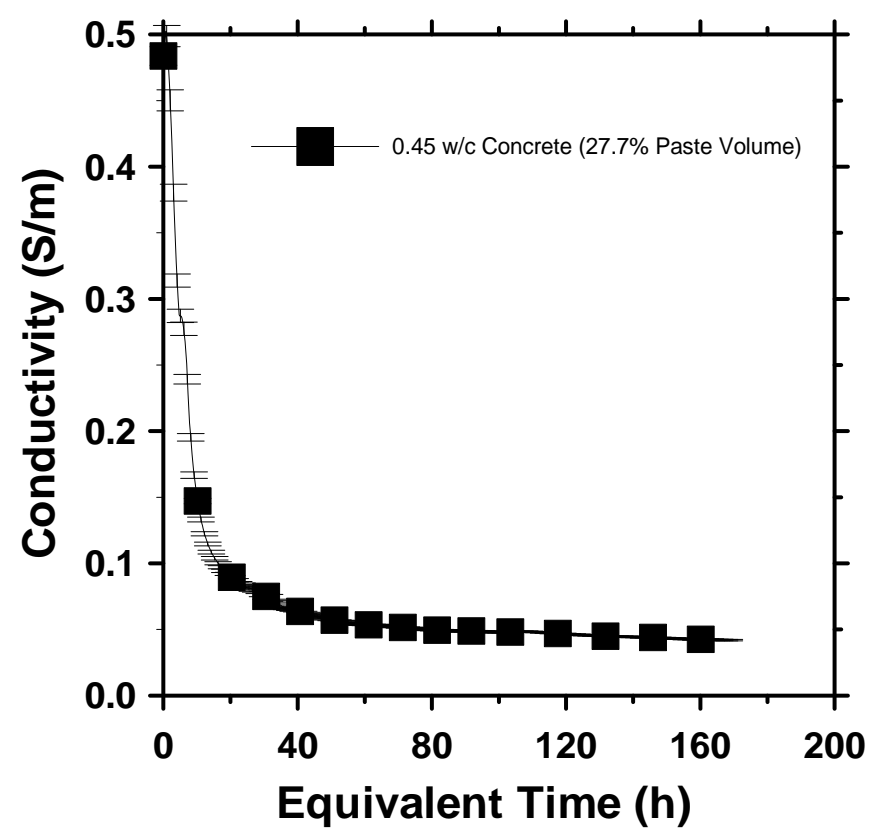




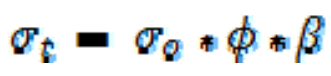

1

2

3

4

5

6

7 the hydration process. As the hydration of cement occurs, chemical changes occur in the

8 pore solution as it becomes more concentrated. Further, as hydration progresses the pore

9 fluid volume fraction decreases, $\varphi$. While the pore fluid volume is decreasing, it is also

10 become more concentrated as the ions in solution have less fluid to occupy making the

11 conductivity of the pore fluid, $\sigma_{0}$, increase. Lastly, the connectivity factor, $\beta$, of the

12 parallel model will change over the hydration process. When the concrete is in the plastic

13 state, ion mobility will be very easy through the sample due to the fact that there are very

14 little obstructions. However, as the hydration products start to form a pore structure, ion

15 transport paths will become much more tortuous and disconnected, making it much more

16 difficult for ions to move within the system, decreasing the connectivity factor. A

17 qualitative understanding of the components of the parallel model is understood, 
1 however, one of the main goals of this research is to quantify how the change each of

2 these components affects the overall system.

4 To determine the components of the modified parallel law it is essential that we

5 consider how these components evolve during hydration. Initially it may be tempting to

6 simply think of these parameters in terms of their change as a function of time; however

7 it is likely more fundamentally appropriate to consider them as a function of the degree of

8 hydration. Conceptually the degree of hydration can be thought of as the change in the

9 volume of water or unreacted cement. A degree of hydration of 0 means that none of the

10 cement has reacted while a degree of hydration of 1 implies that all of the cement has

11 reacted.

\section{7.6.1.1 Degree of Hydration}

13 Isothermal conduction calorimetry was used to estimate the degree of hydration

14 for the mixtures described in this report. Isothermal calorimetry experiments were 15 conducted on 2 samples of each of the four pastes that reflect the w/c of each of the EIS 16 samples: $0.36,0.42,0.45$, and 0.50 .

17 An isothermal conduction calorimeter is a device that measures how much energy 18 is released by a chemical process; in this case, the hydration of cement. The amount of 19 energy released during the cement hydration can be related to the degree of hydration of 20 the cement if the ultimate heat that is liberated by complete hydration of the cement is 
1 known. While this may be difficult to calculate it can be estimated as $475 \mathrm{~J} / \mathrm{g}$ for the

2 cement used in this study (Castro et al. 2010b).

3 The key assumption that is being made when correlating heat released at a certain

4 time and degree of hydration is that all the constituents of the cement are hydrating to

5 provide an average rate of hydration. The calorimeter returns a value of cumulative

6 energy over time as shown in Figure 7.7. For this energy to be converted to a degree of

7 hydration, the theoretical maximum energy of hydration must be calculated. This can be

8 done for a specific cement using a copy of the mill certification. The mill certification

9 will give a value of how much of each constituent is in the cement. From there, the

10 maximum amount of energy each constituent could produce during the reaction is

11 calculated from thermodynamic properties. The value of total energy released at any

12 time over the total maximum possible energy released is the degree of hydration value.

13

14

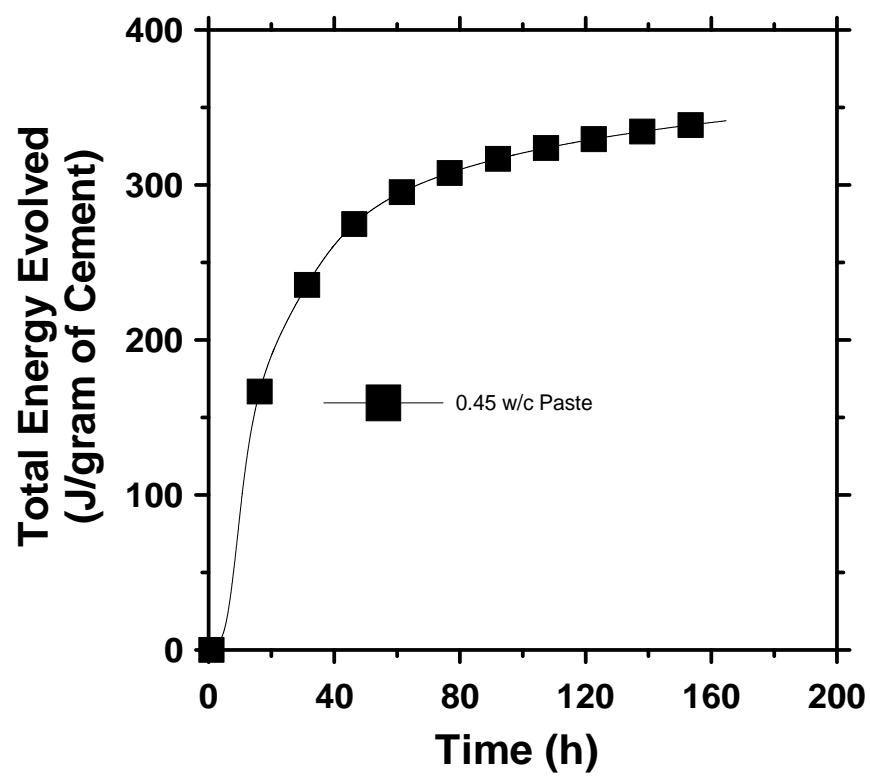

Figure 7.7: Total Energy Evolved from Calorimeter 
2 the cement paste as shown in Figure 7.8.

$11{ }^{\circ} \mathrm{C}$ oven for 24 hours. Then a known mass of sample is placed in a crucible of known

12 mass. The sample is placed in a muffle furnace at $1050{ }^{\circ} \mathrm{C}$ for three hours and then

13 weighed. The difference in the sample weight before and after the muffle furnace

mod consisted of crushing the cement paste into a fine powder and immersing this

dust in acetone to extract any pore fluid from within the pores to stop the hydration

9 process. The sample is then let sit in the acetone for 24 hours and then placed in a dish

0 for 6 hours to let the acetone evaporate off. The then dry sample is further dried in a 105

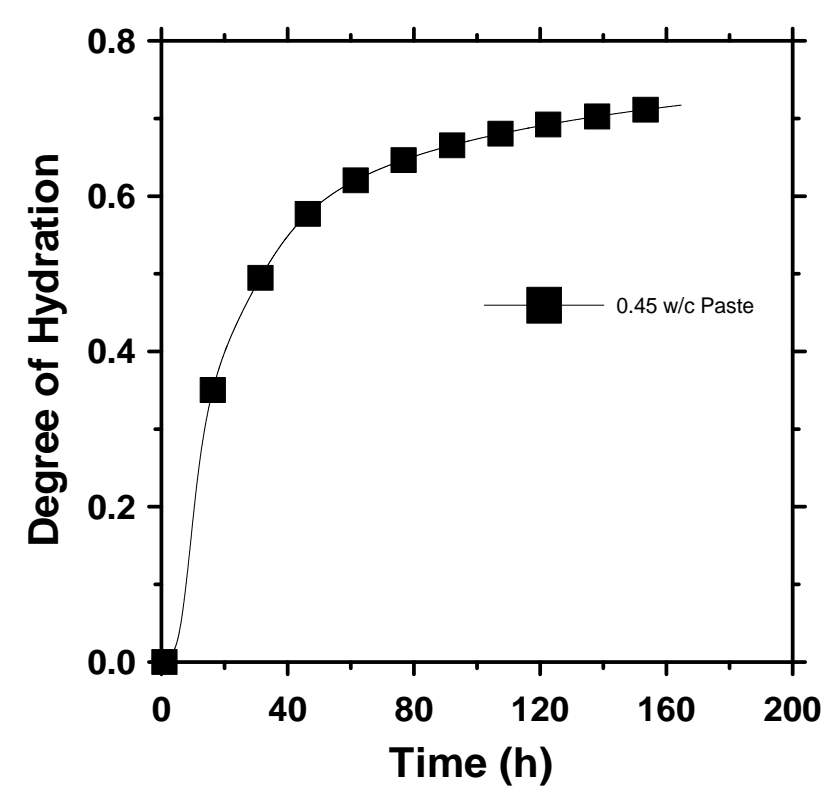

Figure 7.8: Degree of Hydration for 0.45 Paste 
1 ignition is the loss of non-evaporable water, which, through Powers model can be related 2 to degree of hydration.

4 The pore solution extractions were performed on samples that were small enough

5 to be considered isothermal, therefore there is no need to apply and equivalent time

6 maturity correction to the pore solution conductivity data. Using the degree of hydration

7 to measure a process can also give a better idea of what changes are occurring in the

8 structure itself. To get a graph of pore solution conductivity vs. degree of hydration, the

9 respective values at equal ages from Figure 7.8 and Figure 7.9 are compared and plotted

10 in Figure 7.10. It is seen from this plot that the pore solution conductivity of a cement

11 paste is linearly related to the degree of hydration of the sample. This is a key trend

12 when trying to quantify the relationships of all the different components of the parallel

13 model. 


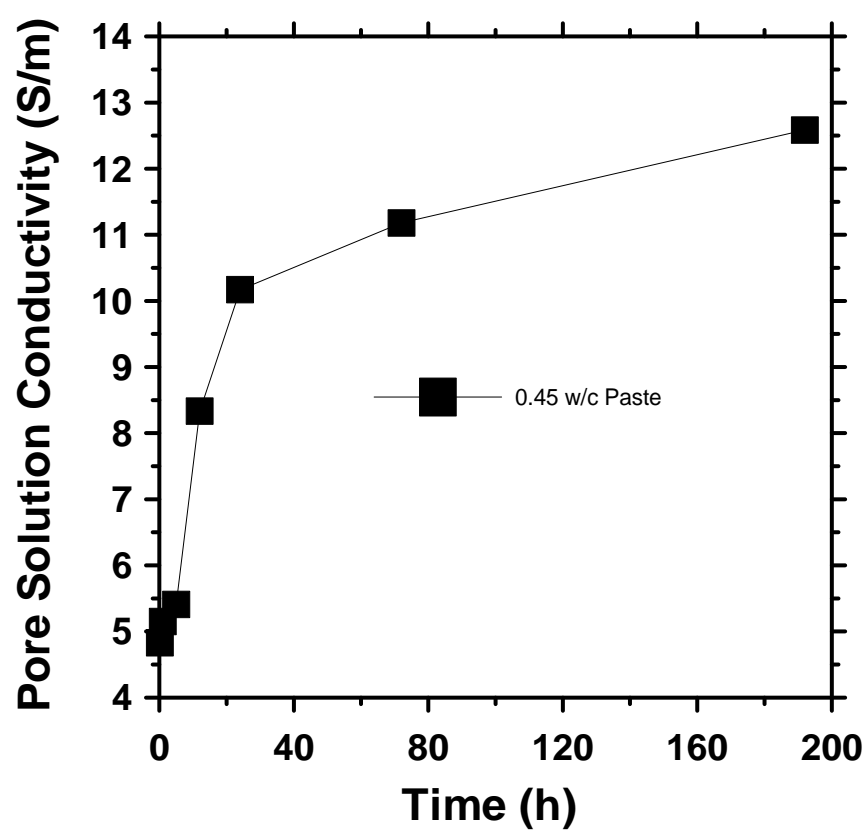

Figure 7.9: 0.45 w/c Pore Solution Conductivity vs. Time

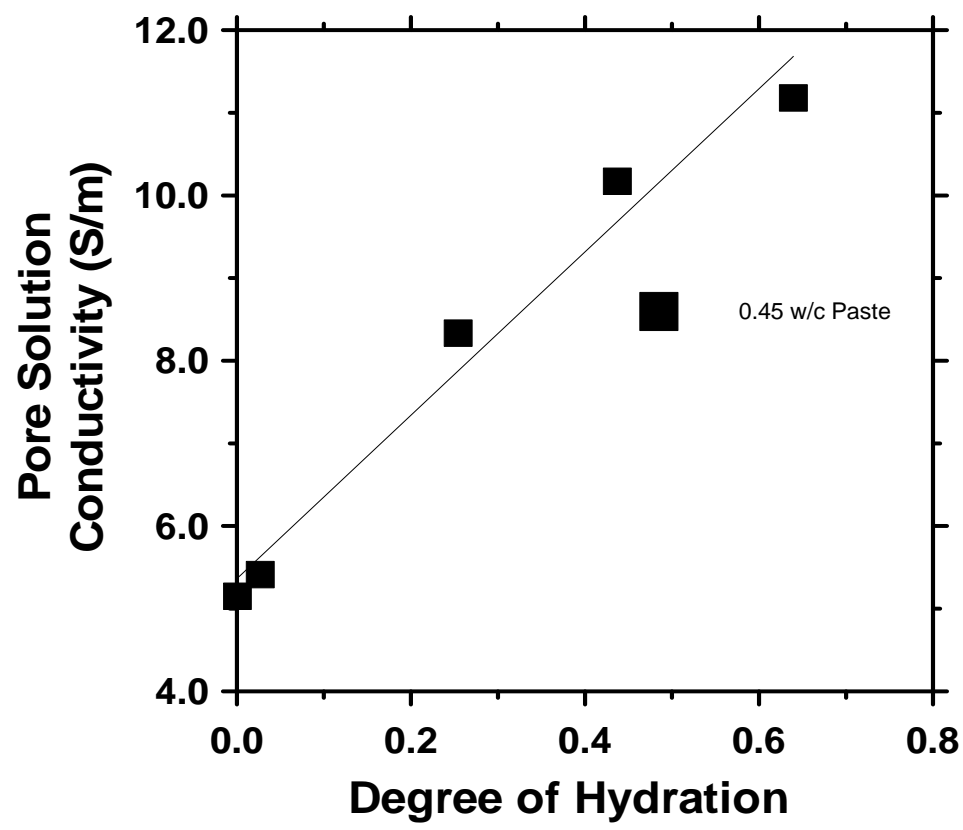

$4 \quad$ Figure 7.10: Pore Solution Conductivity vs. DOH for a Paste with a w/c of 0.45 
6 simple QC/QA use. Ideally, one would be able to measure the change in conductivity of

7 the pore solution over the hydration process, in real time. Rajabipour (2006) has made a

8 huge step towards this goal with the development of the Pore Solution Conductivity 9 Sensor.

12 chosen for its pore size and pore distribution. Its unique pore structure allows

13 surrounding pore solution to diffuse into the system, making the monitoring of real-time 14 changes in the pore solution possible.

15 The concept of the sensor follows the same logic as the EIS, where a saturated 16 material has a potential applied to it and a resistance is calculated. A small, prismatic, 17 sample of the stone, $8 \mathrm{~mm} \times 8 \mathrm{~mm} \times 2 \mathrm{~mm}$, has electrodes attached to each of the $8 \mathrm{~mm}$ 18 faces of the sample. These electrodes are covered with epoxy to eliminate the short 19 circuit that would develop (Figure 7.11). From there, the sensor is dried to $1 \% \mathrm{RH}$, then 20 vacuum saturated in a 0.4 molar potassium hydroxide solution. After these steps are 21 carried out, the sensors are ready to be placed in a fresh sample. 


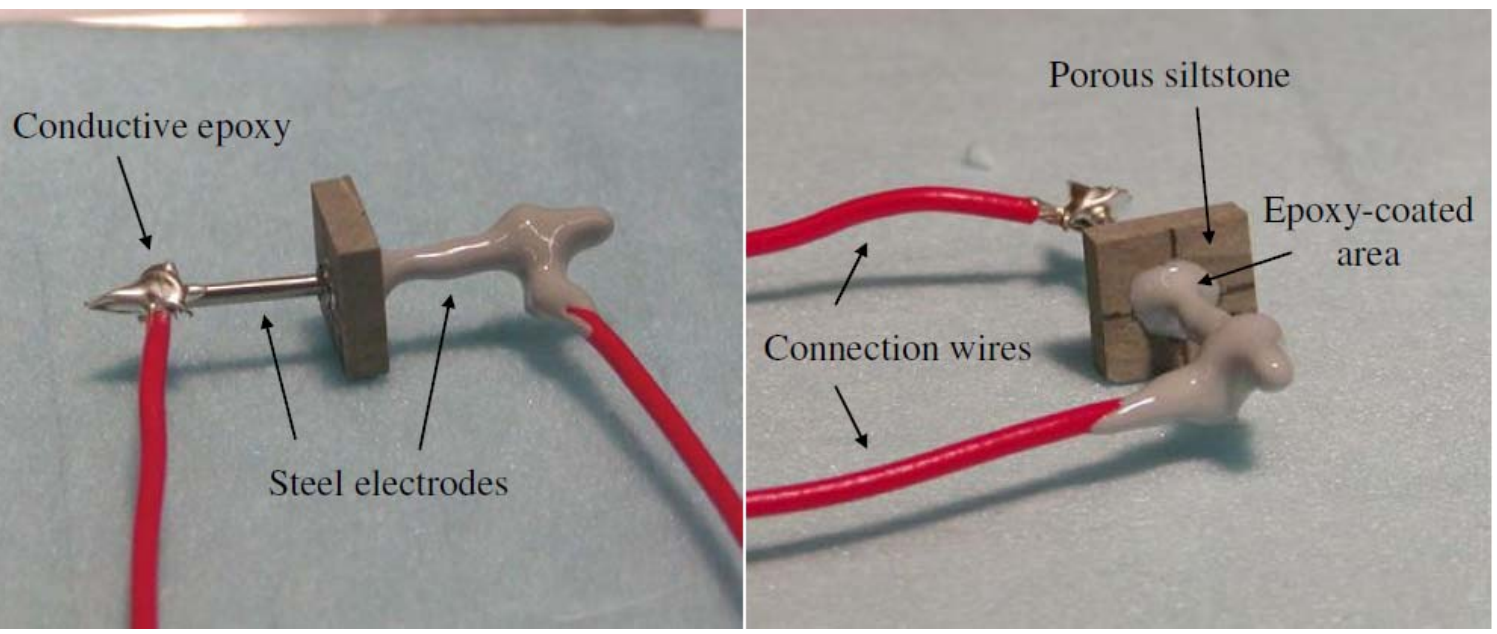

2 Figure 7.11: Front and Side View of the Rajabipour Developed Pore Solution Sensor

When EIS is applied to a concrete sample immediately after mixing, the system is

4 undergoing radical changes as the cement is undergoing hydration; the fluid volume is

5 decreasing, the pore solution is becoming more concentrated, and the pore network is

6 developing. Conversely, as long as the pore solution sensor stays saturated, the pore fluid

7 volume fraction and pore network are unchanging. The only variable in the pore solution

8 sensor system is the pore solution conductivity. A typical plot of the sensor data can be

9 seen in Figure 7.12. It is displayed as a function of the pore solution conductivity that

10 was acquired in the research discussed in 7.6.2. The acquisition of the data collected by

11 the sensor began within an hour of casting of the sample and ran at half hour intervals for

12 one week. The sample the sensor was imbedded in was a $0.45 \mathrm{w} / \mathrm{c} 45 \%$ paste by volume,

13 mortar. 


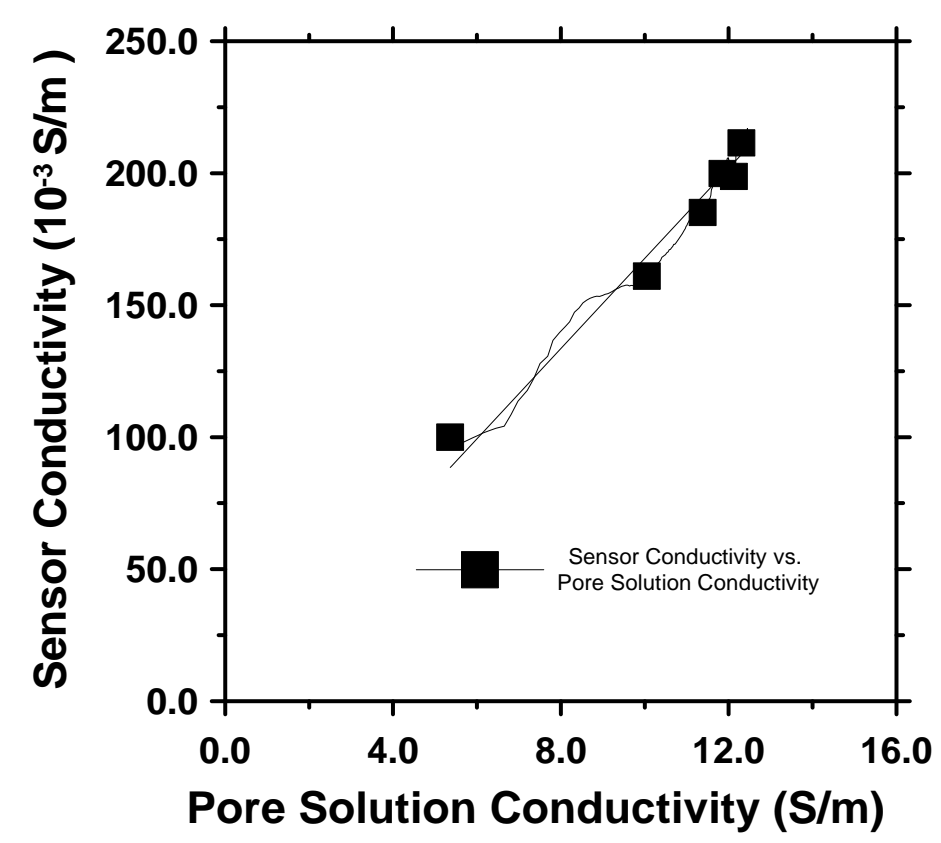

4 performed by Rajabipour. However, the slopes of the linear fit lines from the experiment

5 were similar in value with this experiments slope as 0.017 and Rajabipour's as 0.015 .

6 Further research is needed to determine the repeatability of the pore sensor as well as its

7 validity over a range of different mix designs.

9 The EIS concrete samples are a sealed system. The degree of hydration data can

10 be used to calculate the pore fluid volume fraction.

11 The pore fluid volume fraction itself can be defined as any fluid in the system that

12 can be used to transport ions in the system. This includes both fluid in the capillary pores

13 and the fluid within the gel pores. 
1 Using the equations developed by Jensen, a pore fluid fraction vs. degree of

2 hydration graph was developed and can be seen in Figure 7.13. This pore fluid fraction is

3 for the paste component only. However, concrete is composed of two other components;

4 air and aggregate. The pore fluid volume fraction of a concrete can be changed greatly

5 depending on the volume of aggregate found in your mixture design. Figure 7.14 shows

6 the volume fraction of the conductive phase in concrete.

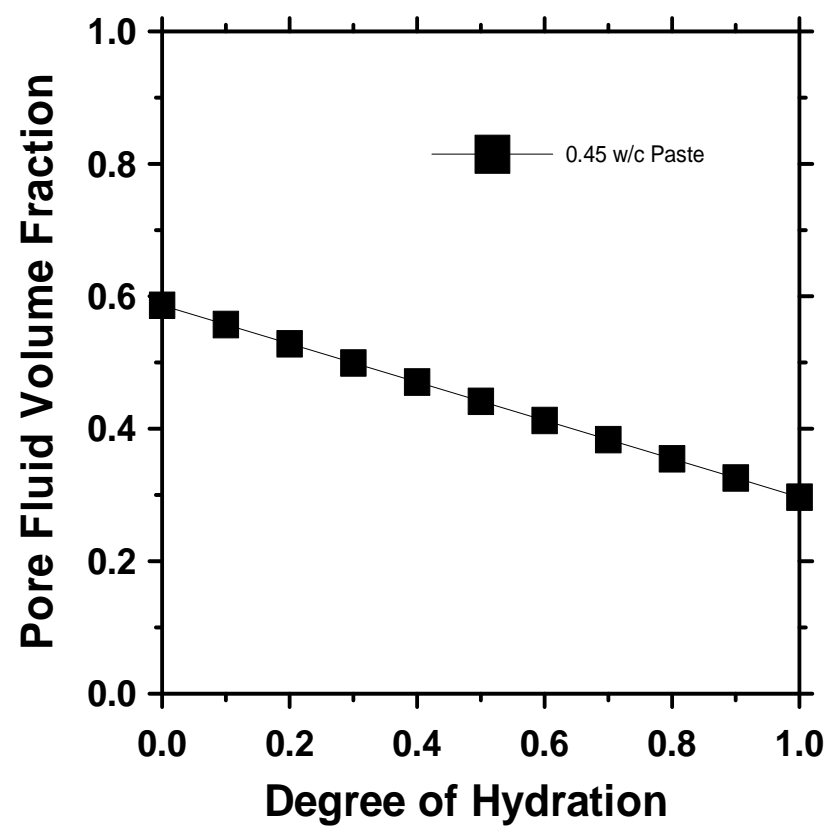

$8 \quad$ Figure 7.13: Pore Fluid Fraction vs. Degree of Hydration for $0.42 \mathrm{w} / \mathrm{c}$ Paste 


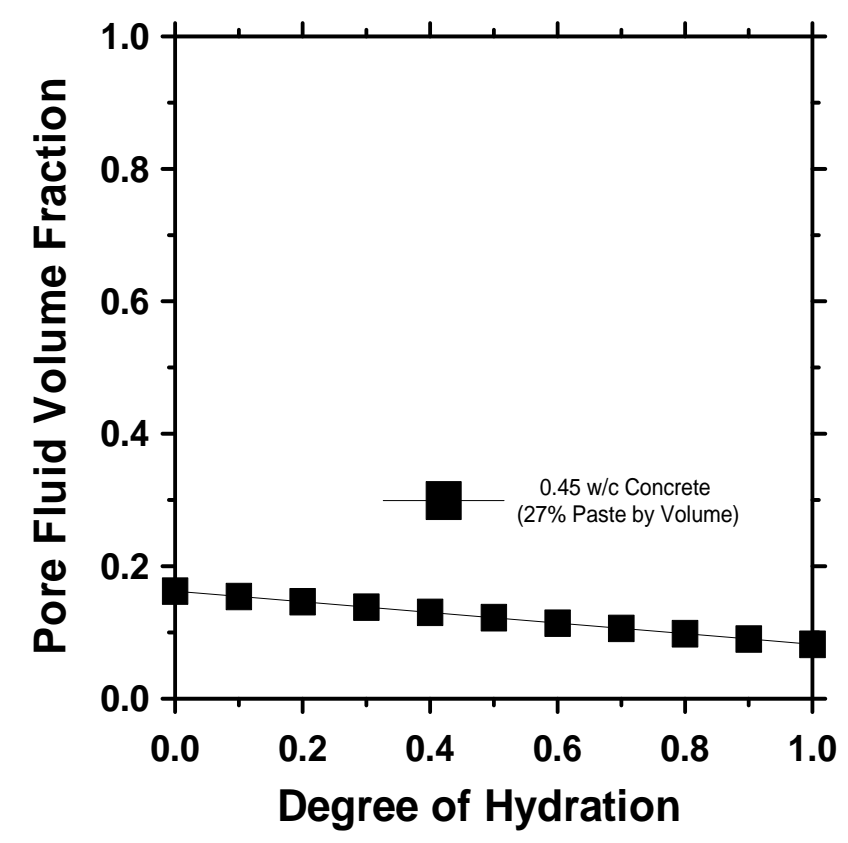

2 Figure 7.14: Pore Fluid Volume Fraction vs. DOH for a 65.7\% Agg. Fraction 0.42 w/c

Concrete

\subsubsection{Effect of Temperature on Pore Solution and Concrete Conductivity}

5 A change in the temperature of a pore solution has a profound effect on the

6 conductivity of the pore solution. Due to the heat of hydration experienced when a

7 cement grain undergoes hydration, the system always undergoes a temperature change in

8 ambient conditions. However this change in conductivity due to temperature can be

9 monitored and expressed through mathematical equations, primarily a modified version

10 of the Arrhenieus equation developed by Sant et al., seen in Equation 7.3 (Sant, 2008).

11 Since the temperature of the EIS samples are monitored throughout the experiment, all of

12 the values are known in Equation 7.3 except for $\mathrm{E}_{\mathrm{ac}}$, the activation energy of conduction.

13 This value can be obtained by performing temperature experiments on the pore solutions

14 samples themselves. 


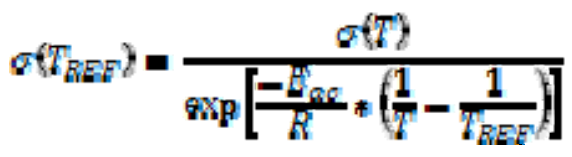

1 where $\sigma\left(T_{E E E}\right)=$ The Conductivity of the Material $(\mathrm{S} / \mathrm{m})$, at the Chosen Reference

2 Temperature $\mathrm{T}_{\mathrm{REF}}\left({ }^{\mathrm{o}} \mathrm{K}\right), \sigma(T)=$ The Conductivity of the Material at Temperature $(\mathrm{S} / \mathrm{m})$, 3 at the temperature $\mathrm{T}\left({ }^{\mathrm{o}} \mathrm{K}\right), E_{\propto \sigma}=$ The Activation Energy of Conduction $(\mathrm{J} / \mathrm{mole})$, and $\mathbb{R}$ $4=$ The Universal Gas Constant $(\mathrm{J} /(\mathrm{K} *$ mole $))$.

5 To obtain the $\mathrm{E}_{\mathrm{ac}}$ for a pore solution, the resistance of the pore solution must be 6 measured at three chosen separate temperatures. The resistances are converted to 7 conductivities through Equation 7.1. Then, because of the relationships of the Arrhenius 8 Equation, the natural $\log$ of the conductivities is plotted vs. in inverse of temperature. A 9 line is fit through these points, and the slope of this line gives the value of $E_{a c} / R$. A 10 typical graph created from this procedure can be seen in Figure 7.15. This procedure is 11 repeated for all the different age pore solutions to obtain an $\mathrm{E}_{\mathrm{ac}}$ at the different ages. An 12 interesting trend is found when comparing activation energies of conduction at different 13 times within the same mixture. There seems to be no trend in the change of $\mathrm{E}_{\mathrm{ac}}$ over 14 time. Furthermore, the values of $\mathrm{E}_{\mathrm{ac}}$ are all within a small standard deviation of each 15 other. For this reason, an average of the activation energies of conduction was 16 calculated, and this value was used as a constant over time for a specific mixture. The 17 range of values can be seen over the different w/c samples tested in Table 7.7. It can be 
1 seen that there is no trend across the different w/c which leads one to believe that the 2 activation energy of conduction is a constant for a specific cement.

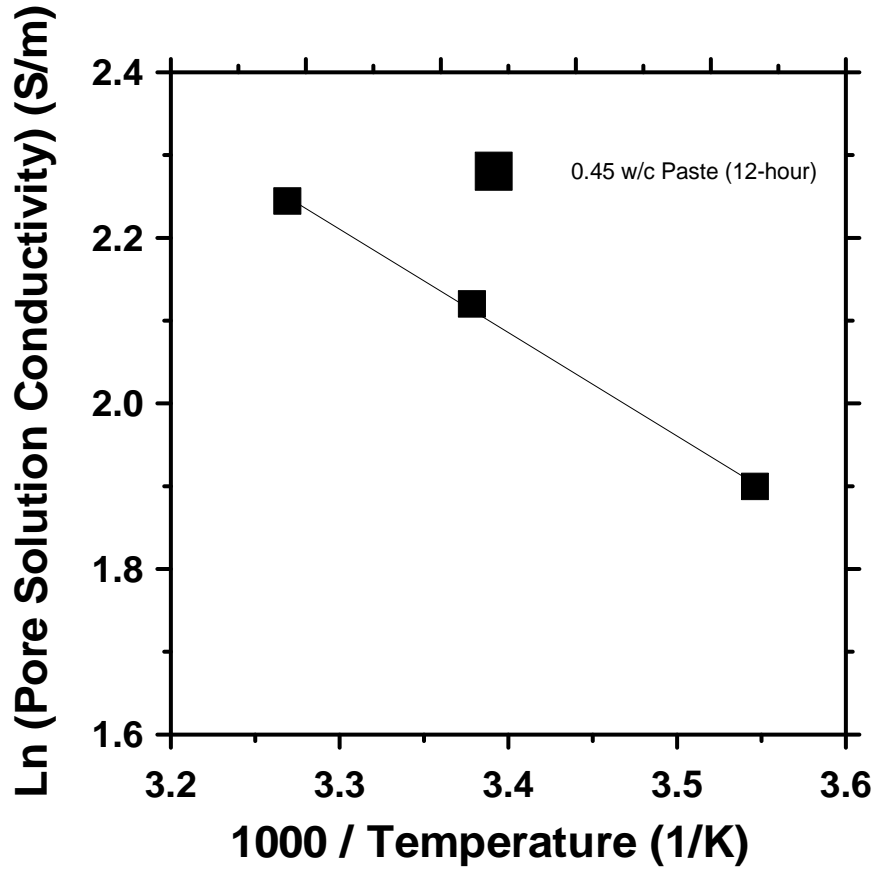

4 Figure 7.15: Natural Log of Pore Solution Conductiviy vs. the Inverse of the

Temperature

\begin{tabular}{|c|c|}
\hline $\mathrm{w} / \mathrm{c}$ & $\mathrm{Eac}(\mathrm{KJ} / \mathrm{mole})$ \\
\hline 0.36 & 9.39 \\
\hline 0.42 & 10.19 \\
\hline 0.45 & 10.06 \\
\hline 0.50 & 9.69 \\
\hline Average & 9.83 \\
\hline SD & 0.36 \\
\hline
\end{tabular}


1 Now that the activation energy of conduction is known, the temperature

2 correction for conductivity can be applied to the EIS data. The process starts with the

3 raw data that is acquired by the EIS experiment that can be seen in Figure 7.16. Because

4 of the increase in temperature of the samples due to heat of hydration, an equivalent time

5 must be calculated before the temperature correction can be employed. After the

6 equivalent time calculation is complete, the temperature correction can be applied to the

7 EIS data.

$8 \quad$ By looking at Figure 7.17, the end of the dormant stage of hydration can see by a

9 sharp increase in the conductivity around five hours, and characterized over time as a

10 "bump" in the data. Again, this is due to the increase in temperature during the

11 acceleration period. When looking at Figure 7.18, which has been corrected for

12 equivalent time and temperature effects, the bump has been almost completely removed.

13 This bump has been claimed to be an effect of many different chemical processes,

14 however, through this research, it seems to be only a temperature effect. 


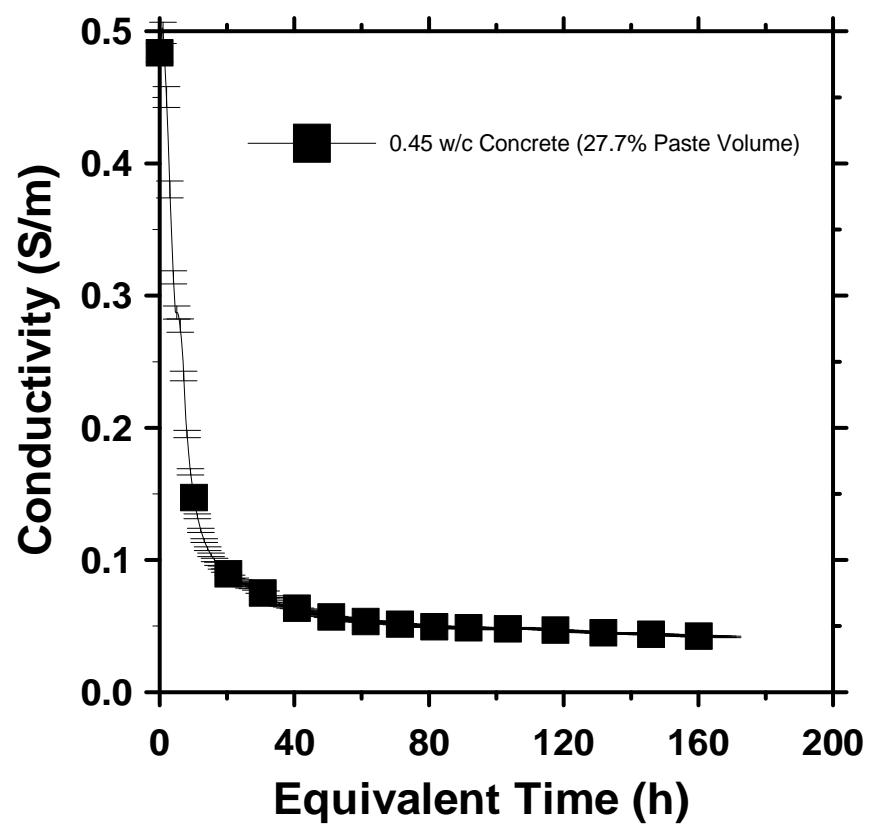

Figure 7.16: 0.45 w/c 27.7\% Raw EIS Data

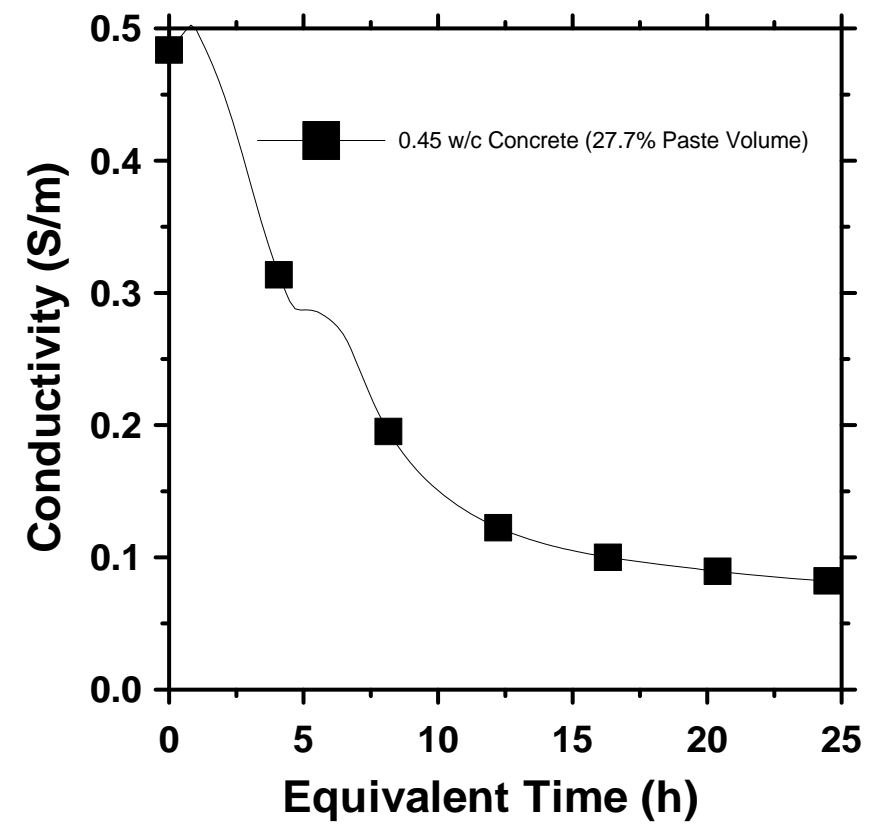

Figure 7.17: 0.45 w/c Concrete (27.7\% Paste Volume) - Early Age Data 


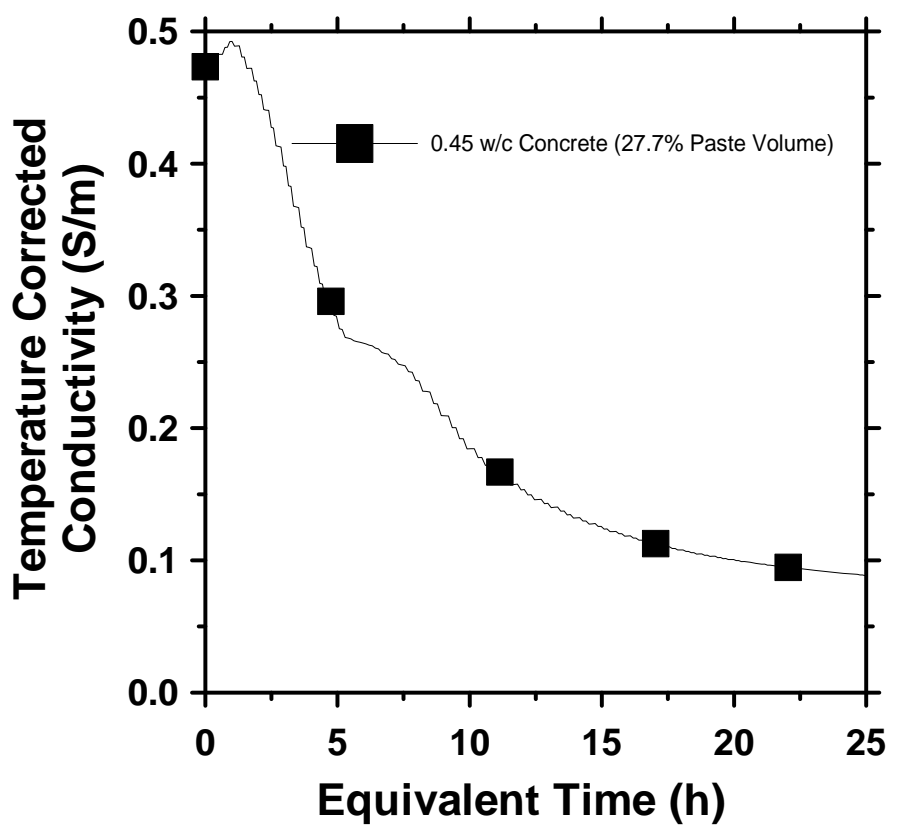

Figure 7.18: 0.45 w/c (27.7\% Paste Volume) - Time and Temperature Corrected

Conductivity Data - Early Age

5 With three out of four elements of the parallel model equation known, $\sigma, \sigma_{0}$, and $\varphi$, the

6 connectivity factor, $\beta$, can be calculated at any point in the data, giving a better

7 understanding of how the pore structure develops over time. But, another important factor

8 that comes from normalizing the concrete conductivity with the pore solution

9 conductivity is called the formation factor. The pore solution volume fraction and

10 conductivity factor are both unitless values that are indications of how the structure of the

11 concrete is changing. As the cement is hydrating and decreasing the fluid volume, the

12 pore system is becoming much more complex. This is causing both the variables to drop

13 simultaneously. This trend is expressed in Figure 7.19. By normalizing the graph in 
1 Figure 7.19 with the pore fluid volume fraction data, a graph of the connectivity 2 component of the parallel model can be viewed in Figure 7.20.

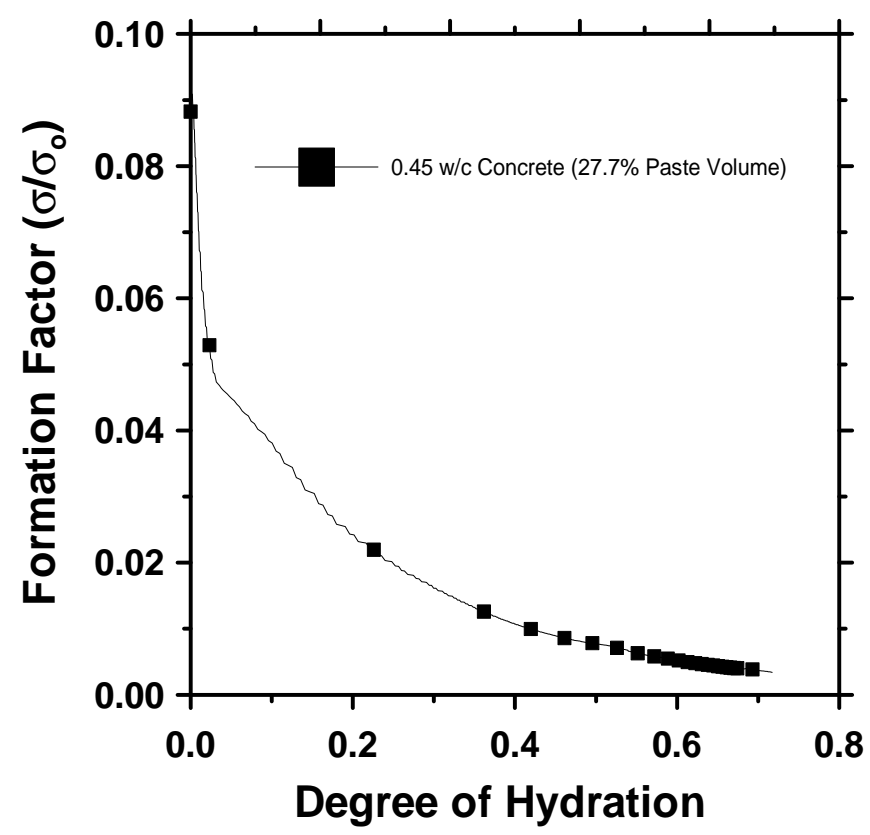

Figure 7.19: Formation Factor vs. Degree of Hydration for a 0.45 w/c 27.7\% Paste

Volume Concrete

6 The connectivity or "beta" factor as it is called, due to the Greek letter used to

7 describe it in the parallel model equation, is a measure of the connectivity of the pore

8 system in a cementitious material. It is a unitless value describing the connectivity of the

9 pores. It ranges in value from 1 to $0 ; 1$ meaning the system is totally connected, without

10 any obstructions, and 0 meaning the substance stops all ion transport.

11 As would be expected, the more difficult it is for an ion has to travel across a

12 material, the lower the conductivity of that material will be. An ion's travel can be made

13 difficult by both the length and size of the path traveled by the ion. As the pore system of 
1 a cementitious system develops, the pores become more tortuous, and less connected to

2 one another, making the path of the ion more difficult over time. Another phenomena of

3 the hydrating process is the pores become more constricted with increasing hydration. As

4 the pore constricts at a point, it creates a "bottleneck" where ions start to crowd allowing

5 a smaller amount of ions to pass through. This decreased flow of ions decreases the 6 conductivity of the system over time.

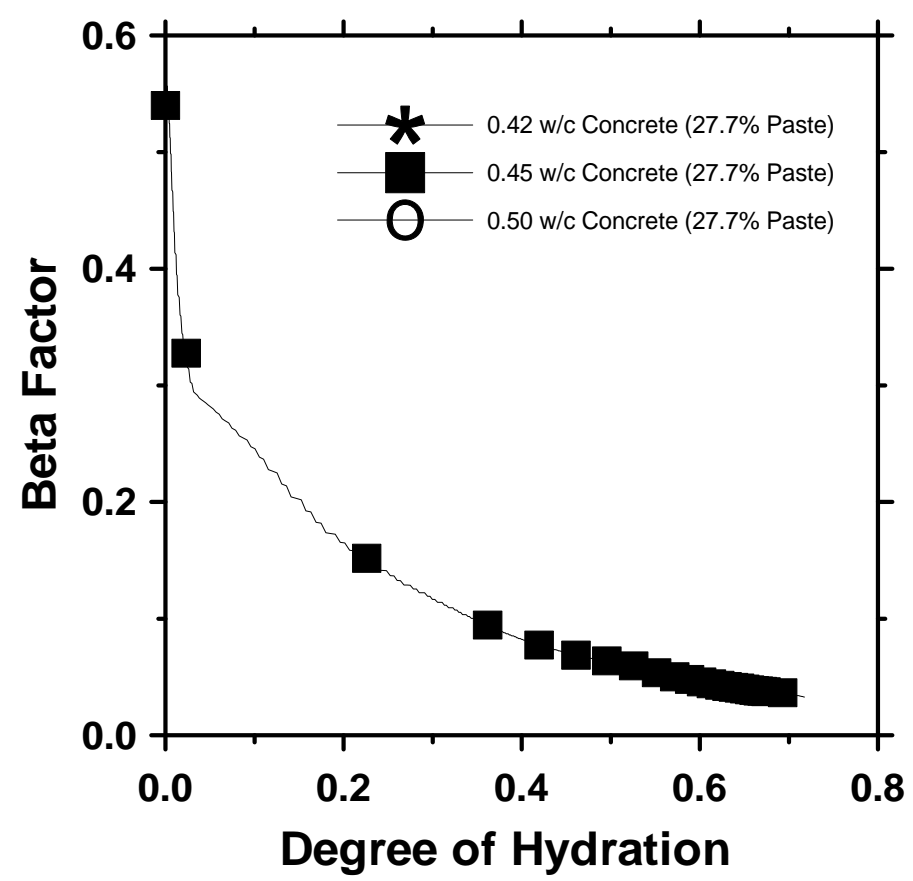

8 Figure 7.20: Beta Factor vs. Degree of Hydration for a 0.45 w/c 27.7\% Paste Volume

Concrete

11 If the EIS procedure explained above is ever to be implemented as a QA/QC

12 practice, a better understanding on how variations of mixture designs affect the results 
1 must be known. The following section is by no means a complete breakdown on the

2 most common changes made to mix design, however it is a good start to see how a

3 variable paste volume, water to cement ratio, basic admixtures, and water content affect

4 the electrical impedance of a concrete system. Certain components frequently added to

5 concrete, such as silica fume, have a profound effect on the EIS results. Further research

6 will need to be performed to test enough of the possible components of concrete to make

7 the EIS a viable choice for QA/QC testing.

10 display how four variables influence the measured electrical response. The four variables

11 are: water to cement ratio, paste volume, water content, and admixture addition.

\subsubsection{Equal Paste Comparison}

13 The first series of tests maintained a constant paste volume but varied the water to

14 cement ratio. Initially, after mixing the difference in the conductivity between the three

15 mixtures is not very significant. The $0.50 \mathrm{w} / \mathrm{c}$ mixture has a greater proposition of water

16 and as a result it has the highest conductivity immediately after mixing. This is followed

17 by the mixture with a w/c of 0.45 and 0.42 respectively. It is approximately around the

18 time of set when the values of conductivity between the three mixtures begin to deviate.

19 At nearly one week, the mixture with a w/c of 0.5 has a $50 \%$ higher conductivity 
1 compared to the mixture with a w/c of 0.42 . This trend can be seen in Figure 7.21 and

2 Figure 7.22.

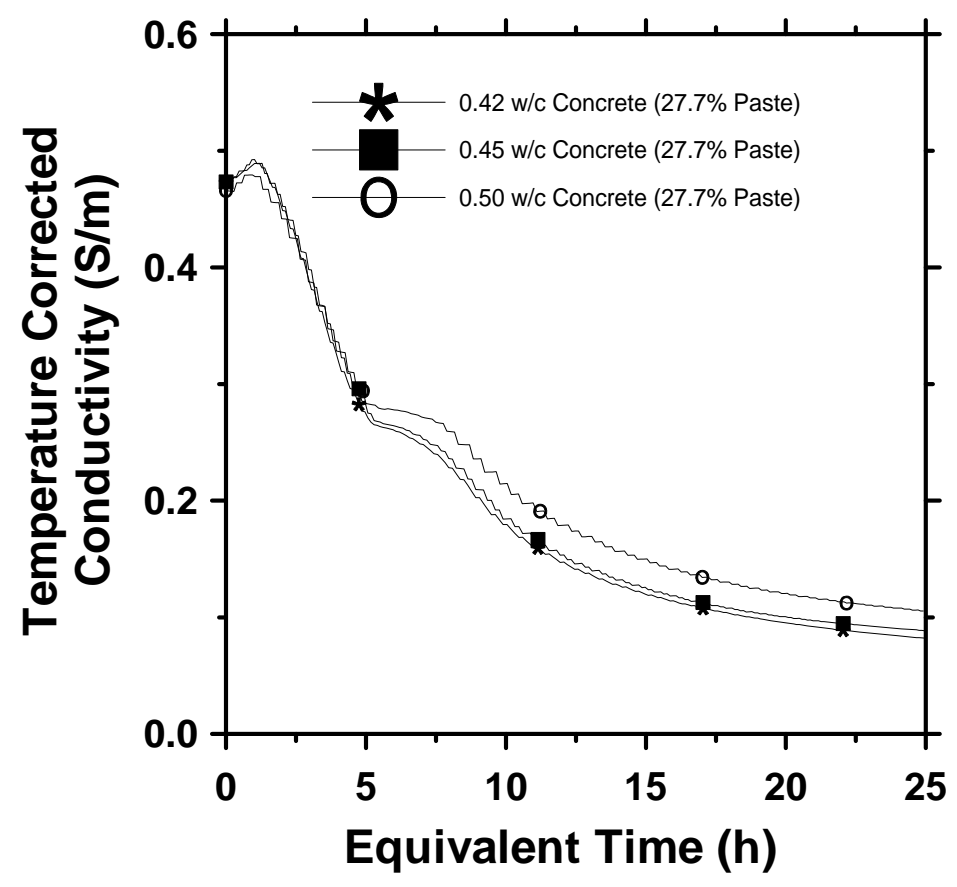

4 Figure 7.21: Temperature Corrected Conductivity vs. Equivalent Time - Early Age 


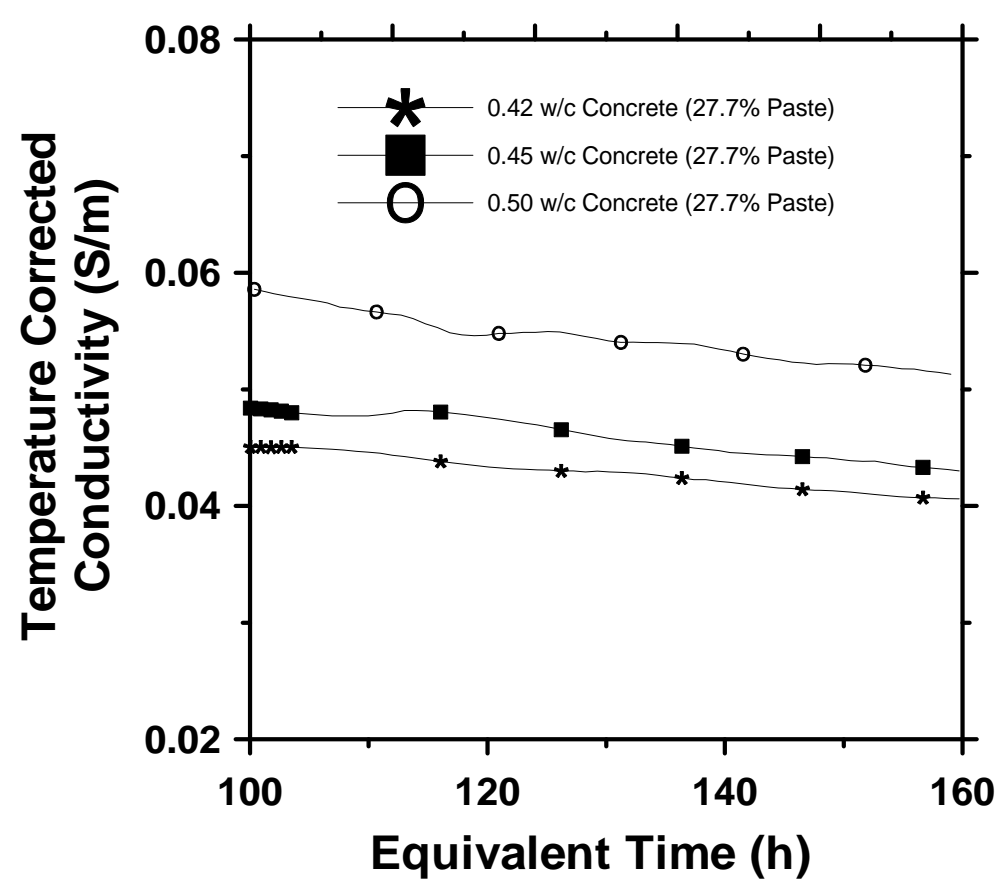

2 Figure 7.22: Temperature Corrected Conductivity vs. Equivalent Time - Late Age

3 Figure 7.23 plots the normalized conductivity of the parallel model as a function

4 of degree of hydration. It can be seen that the system with a higher w/c has a higher

5 conductivity. This is due to higher porosity $(\varphi)$ and a higher connectivity factor $(\beta)$.

6 Powers model can be used to estimate the porosity enabling the $\beta$ factor to be estimated

7 directly as shown in Figure 7.24. 


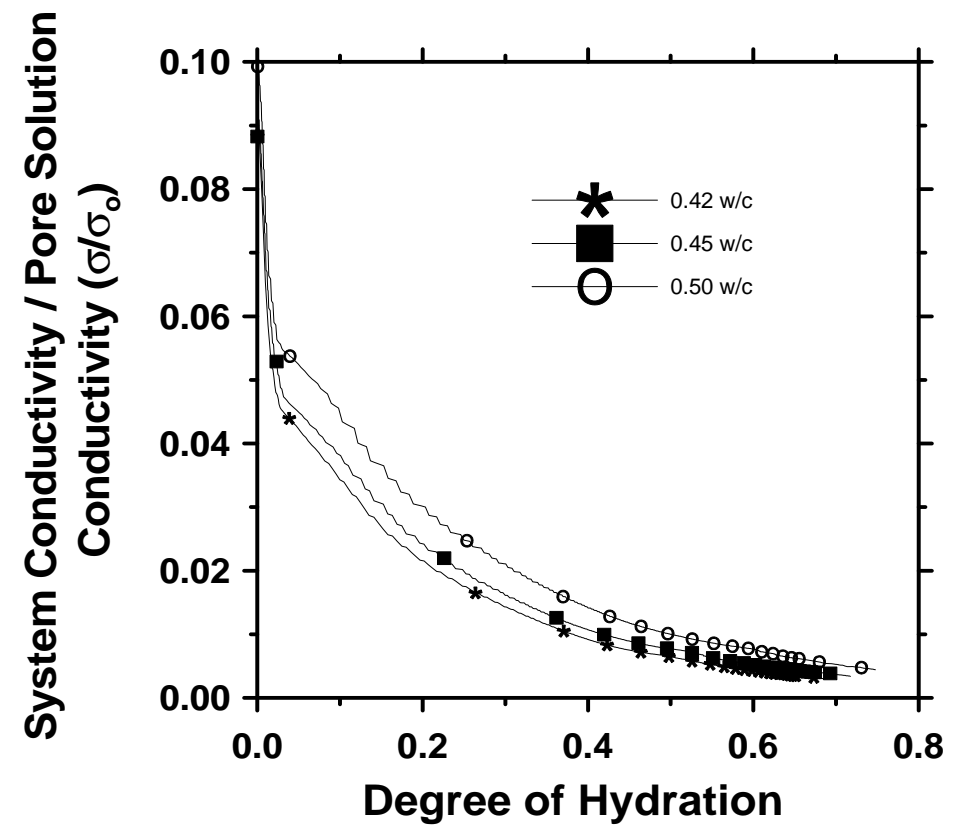

Figure 7.23: Equal Paste Comparison - Normalized Conductivity

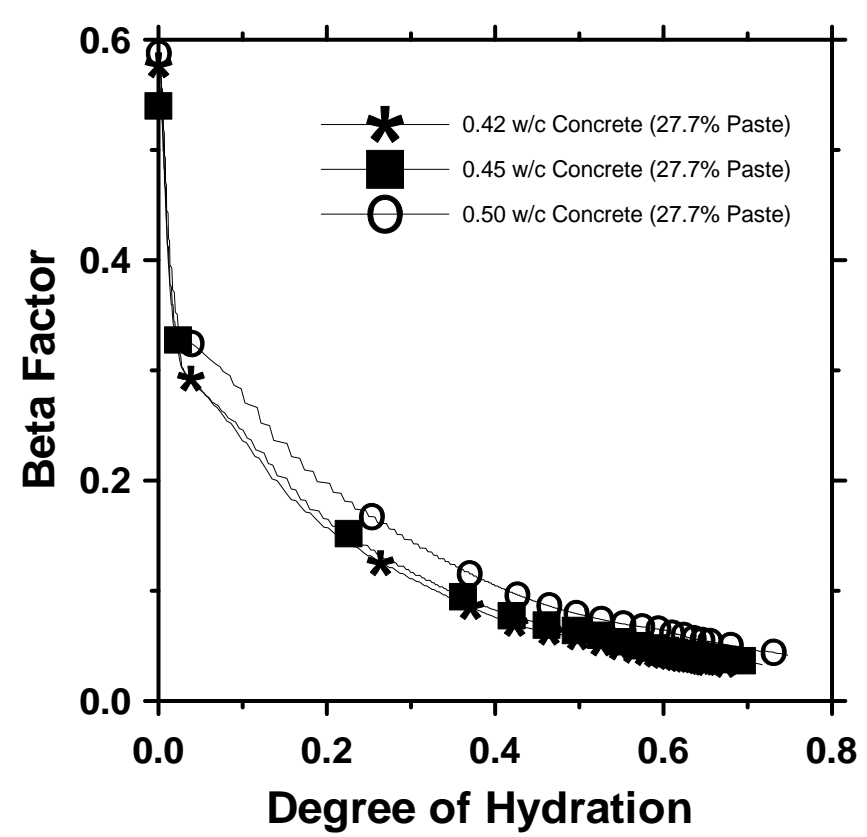

Figure 7.24: Equal Paste Comparsion - Beta Factor 


\subsubsection{Differing Paste Volumes}

2 The second series of experiments were performed to assess the influence of the

3 volume of paste in the concrete. The standard mixture with a w/c of 0.42 has a paste

4 volume of $27.7 \%$, which is a relatively low amount of paste. A second mixture was

5 prepared with a higher paste volume, a w/c of 0.42 paste by volume of $34 \%$. By

6 maintaining the w/c constant between the two mixtures in the experiment, the pore

7 solution conductivity will be the same throughout the hydration process, meaning the

8 other two parameters in the parallel model, $\varphi$ and $\beta$, will be the only factors affecting the

9 results of the experiments.

10 The increased water faction of the mixture with an increased paste volume results

11 in a higher initial conductivity since the pore solution conductivity is equal. This

12 difference is most pronounced at early ages (Figure 7.25) with the increased paste

13 mixture having around a $40 \%$ higher conductivity value compared to the standard

14 mixture. However, as the individual mixtures pass set, the difference in the values has a

15 sharp decrease, then a more steady decrease until a DOH value of 0.5 is achieved as

16 shown in Figure 7.26 when the decrease starts to increase again, presumably due to pore

17 refinement. 


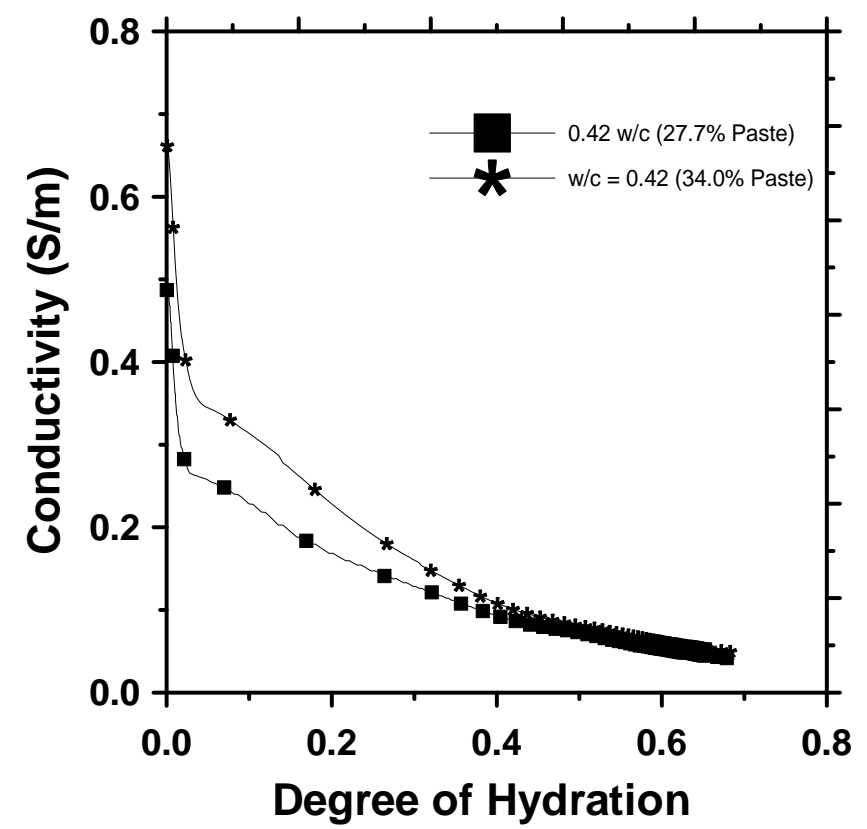

Figure 7.25: Conductivity vs. Degree of Hydration for Equal W/C Concretes with

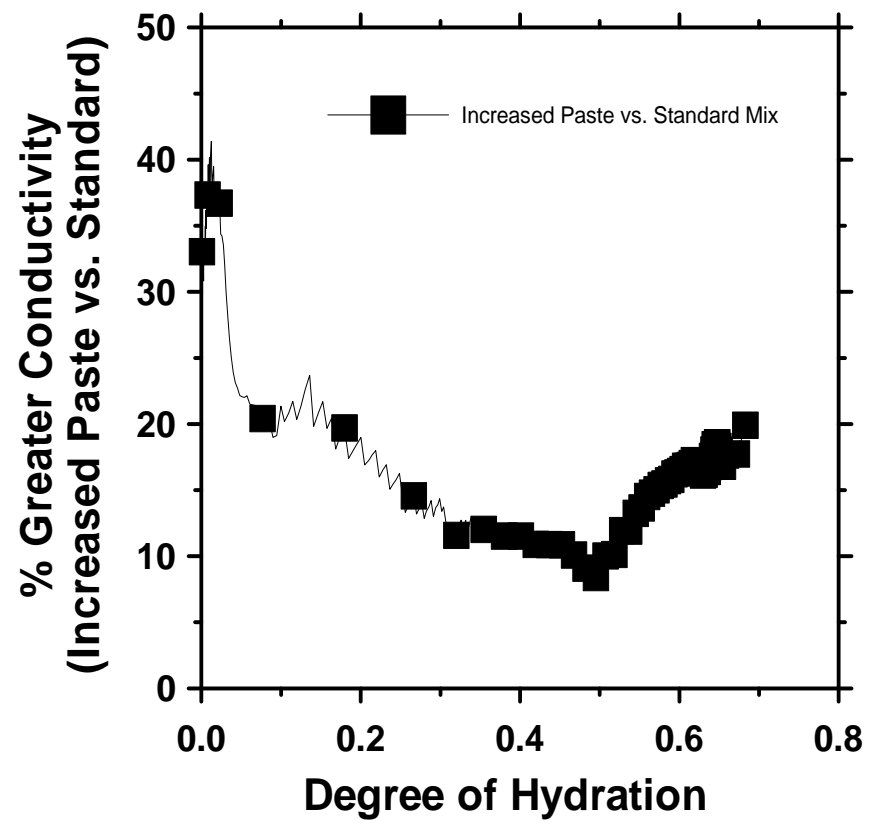

5 Figure 7.26: Percent Greater Conductivity of the Increased Paste Mixture Compared to 6 the Standard Mixture 


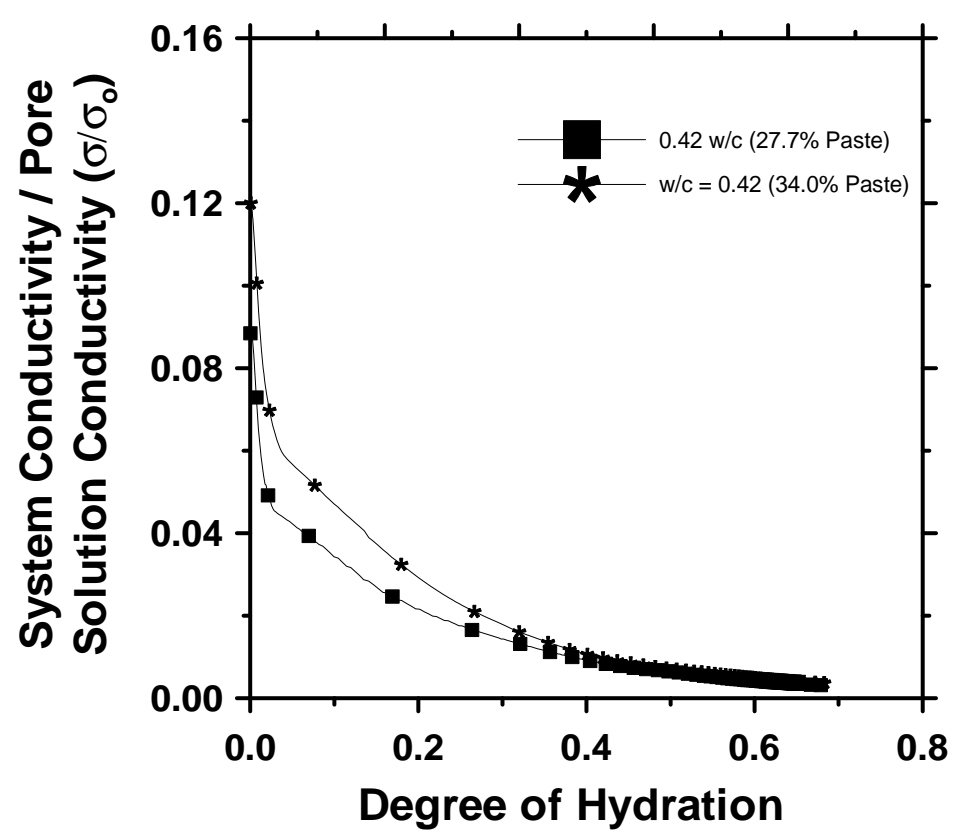

2 Figure 7.27: Conductivity of the System / Pore Solution Conductivity vs. Degree of Hydration for Equal W/C Concretes with Differing Paste Volumes

5 appears to be no correlation between an increased paste volume and connectivity. As can

6 be seen in Figure 7.28, the connectivity factor of the $27.7 \%$ paste samples and the 34.0

$7 \%$ paste samples are virtually identical throughout the process. An increase in the 8 amount of aggregate does little to increase the tortuosity of the pore system. 


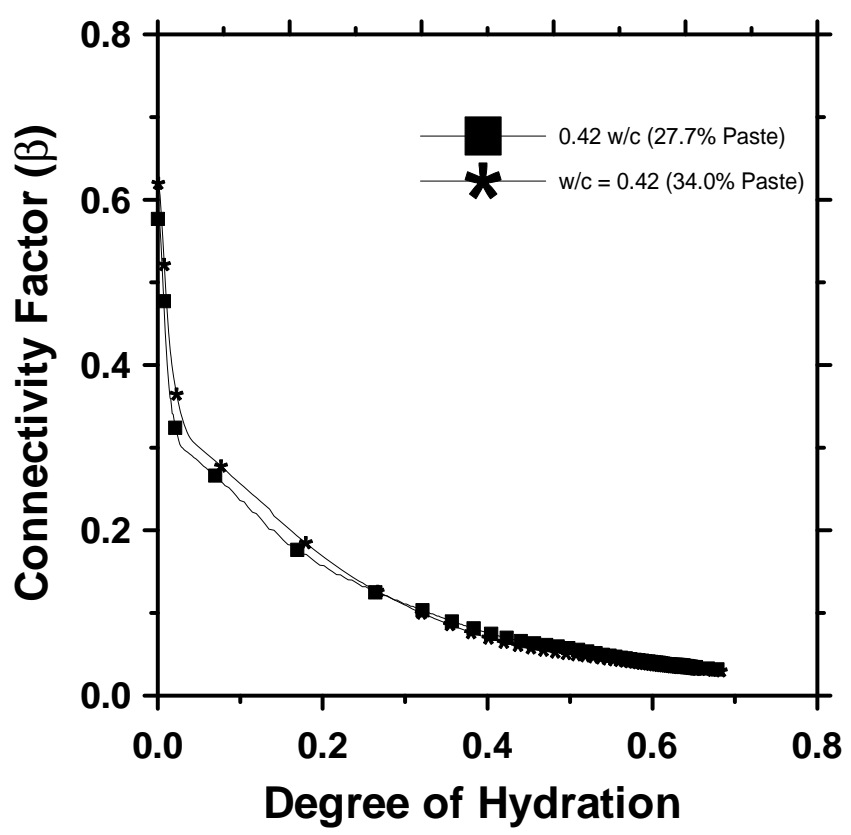

2 Figure 7.28: Connectivity Factor vs. Degree of Hydration Comparison of Equal w/c

Concretes with Different Paste Volumes

The comparison of water content was designed to simulate a situation that could

6 be encountered in the field. If the workability of an already delivered concrete batch

7 needed to be increased, how would adding water to the truck change the transport

8 properties of the mixture compared to the original concrete delivered. The control

9 mixture $(0.42 \mathrm{w} / \mathrm{c}-27.7 \%$ paste $)$ was used. However, additional water was added to the

10 mixture to increase the water to cement ratio to 0.45 and again to 0.50 . This does change

11 the water to cement ratio as well as the paste volume. As one would expect, the

12 increased water in the mixture increases the overall conductivity (Figures 7.29, 7.30, 13 7.31). 


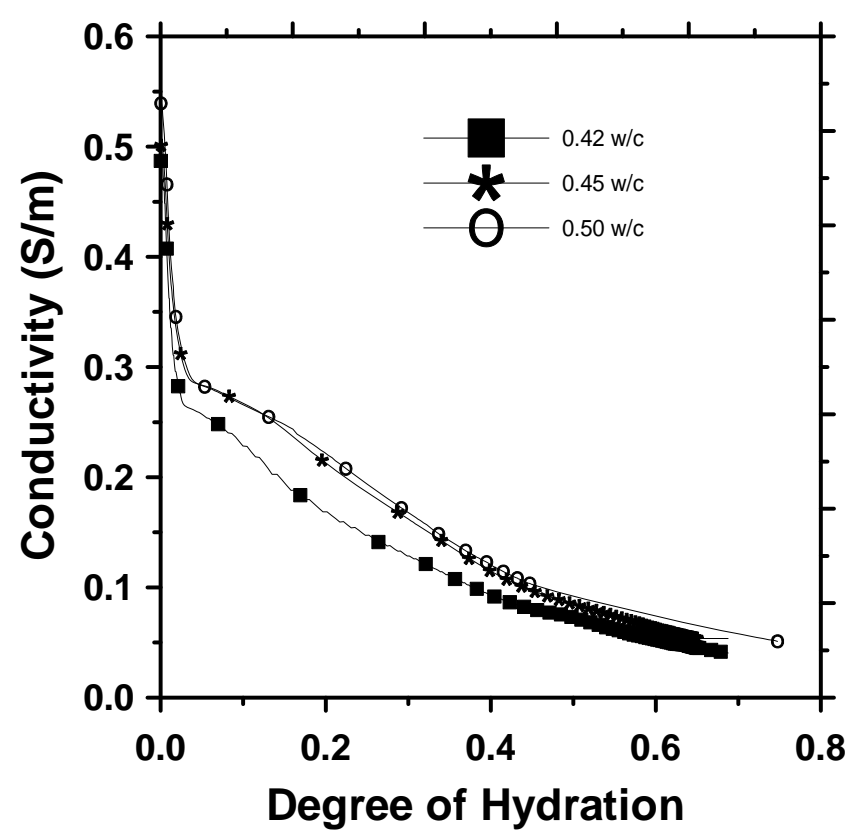

2 Figure 7.29: Temperature Corrected Conductivity vs. Degree of Hydration Over an 3 Increase in Water Content

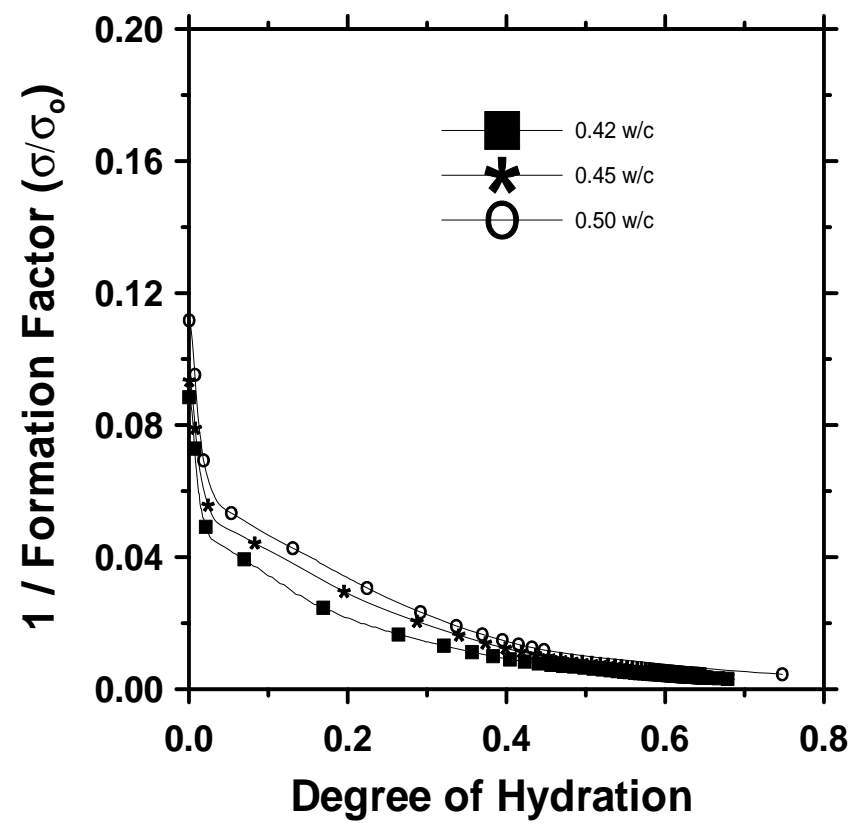

5 Figure 7.30: 1 / Formation Factor vs. Degree of Hydration Over an Increase in Water 6

Content 


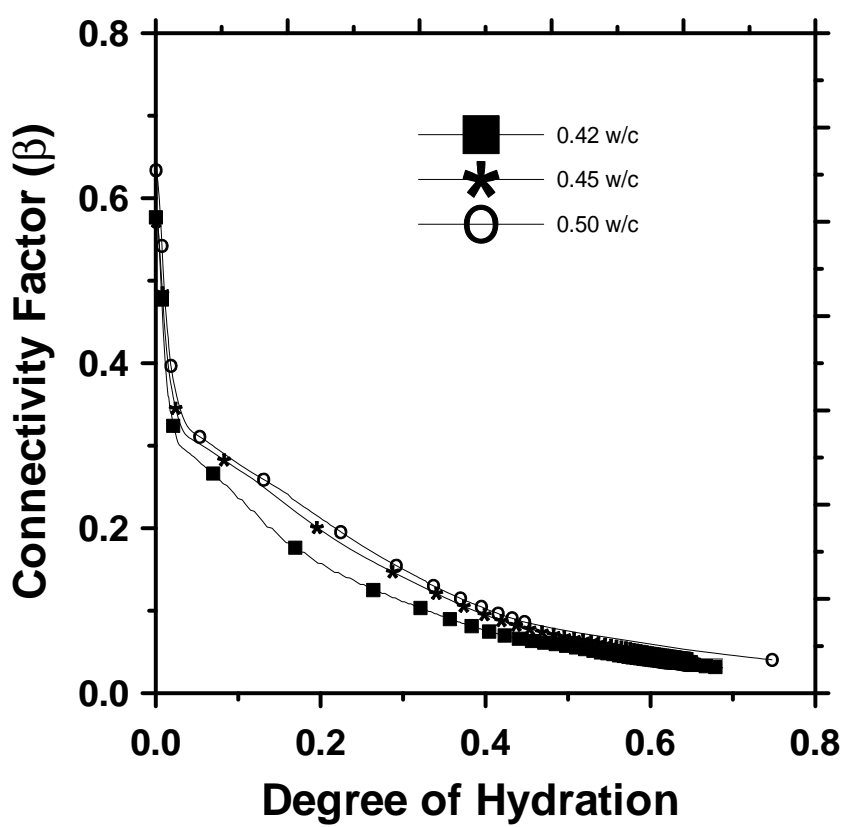

2 Figure 7.31: Connectivity Factor vs. Degree of Hydration over an Increase in Water

An alternative method was investigated to increase workability without the

5 addition of water, namely the use of a water reducing admixture. This is discussed in the

6 following section.

8 The mixture designs compared in the previous section did not have any 9 admixtures within their mixture design. This was to limit the number of variables that 10 could affect the results of the tests. However, nearly all commercial "Ready-Mix"

11 batches contain some sort of admixture. As a preliminary test, an air entrainer, as well as

12 a high-range water reducer were respectively added to the $0.42 \mathrm{w} / \mathrm{c} 27.7 \%$ paste standard

13 mixture. The results can be seen in Figures 7.32, 7.33 and 7.34. 


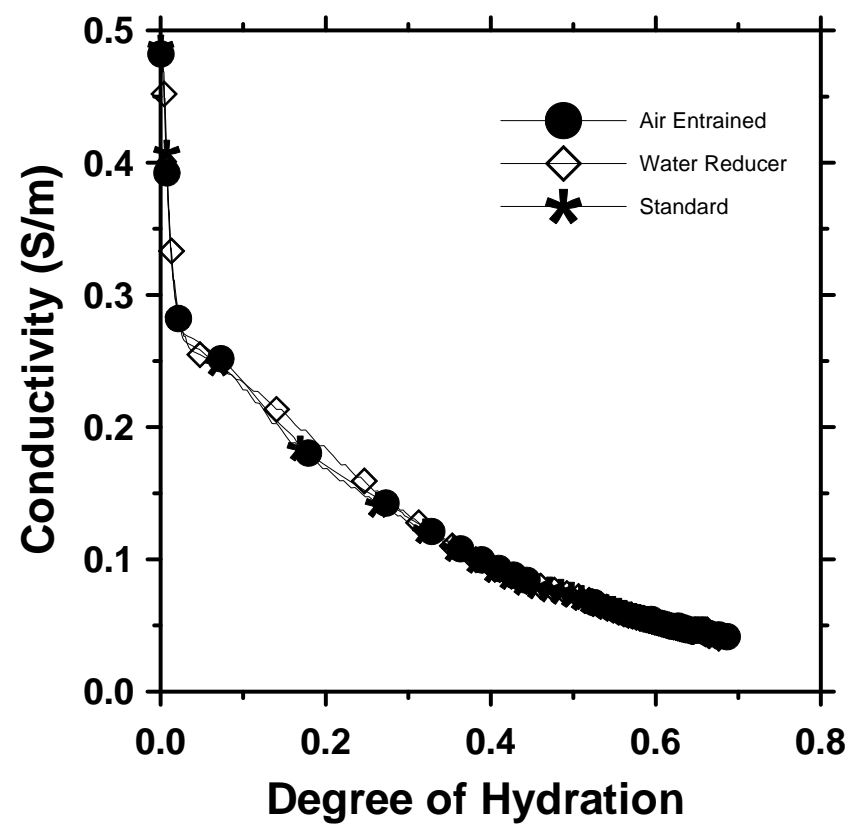

Figure 7.32: Comparison of Conductivity of Samples Containing Admixtures

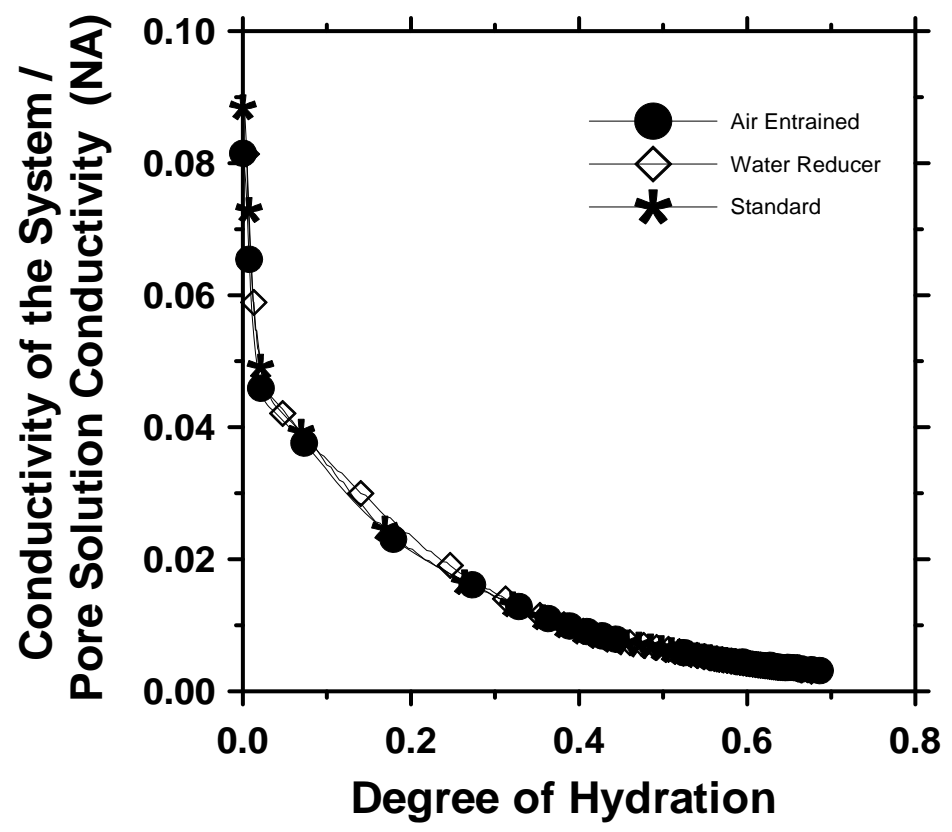

4 Figure 7.33: Conductivity of the System Normalized to the Pore Solution Conductivity 5 of the Admixture Comparison Samples 

9 concrete system.

12 fluid transport properties. The influence of temperature, paste volume and pore solution

Figure 7.34: Connectivity of the System vs. DOH for the Admixture Comparison Samples

It can be seen from all three graphs that neither air entrained nor the high-range water reducer samples deviate from the standard mix by a significant amount. It can be argued that air filled porosity will not transport electrical signal The behavior is the same for all three comparisons, highlighting the fact that in small amount, both air entrainer and high-range water reducer do not change the conductivity or transport properties of a

\subsection{Summary and Conclusions}

This chapter has reviewed the use of electrical measurement as a method to assess

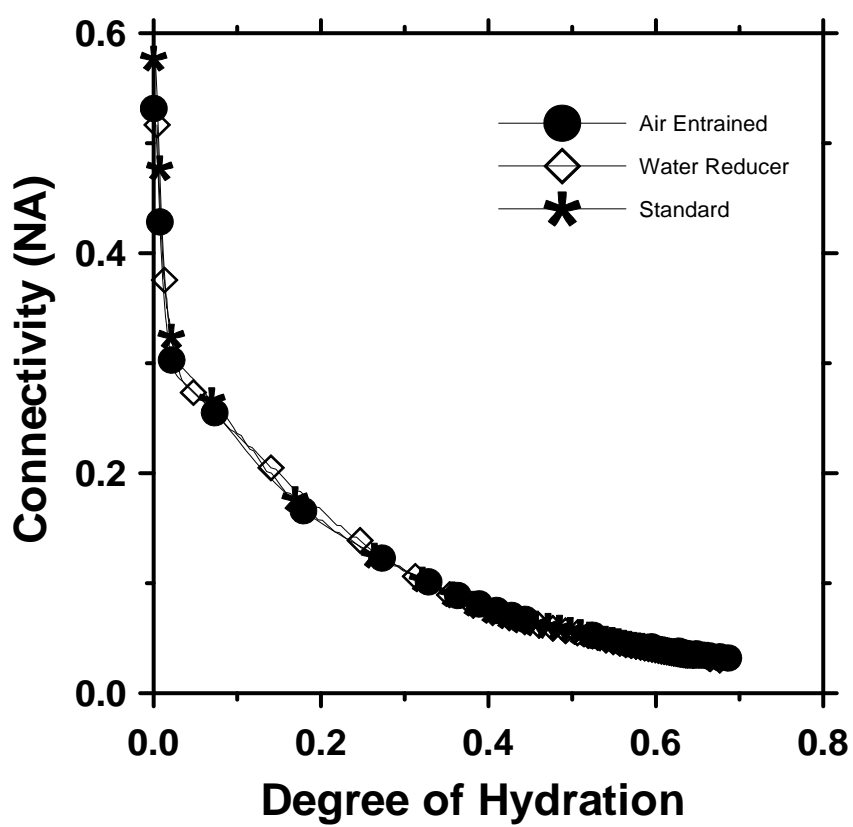


1 conductivity are discussed. This work will be a valuable addition to the background that 2 forms a new standardized method for newer electrical properties. 
To better understand the relative humidity that can be expected to develop in

5 concrete in the field, a series of instrumented slabs were tested with several different

6 exposure conditions. These tests considered the effects of boundary conditions on the

7 wetting and drying of concrete. Six exposure conditions were evaluated including:

8 1) a covered surface,

9 2) an exposed vertical surface,

10 3) a horizontal surface on a drainable base,

11 4) a horizontal surface on a non drainable base,

12 5) a completely submerged sample and

13 6) a slab stored at $23{ }^{\circ} \mathrm{C}$ and $50 \%$ relative humidity.

14 The slabs were prepared using a mixture that was similar to a typical INDOT

15 paving mixture; however hollow cylindrical shafts were placed in the sample to enable

16 sensors to be placed in the sample. By placing sensors at different depths in the exposed 
1 samples, the internal relative humidity $(\mathrm{RH})$ and temperature (T) profile that can be

2 expected in field concrete in Indiana is obtained.

3 The relative humidity of concrete in the field is important for evaluating the long-

4 term durability of this concrete. It is known that if the internal $\mathrm{RH}$ of a concrete drops

5 below approximately $80 \% \mathrm{RH}$, the hydration of cement particles slows substantially.

6 Improper curing can lead to problems such as excessive shrinkage and cracking or poor

7 strength development. Another reason to monitor the relative humidity in a sample

8 which is due to its influence on the moisture transport properties of concretes. If external

9 moisture application to a sample is known, an accompanying change in internal RH can

10 be correlated to the transportation properties of the sample itself (Castro, 2010).

\section{$\underline{8.2 \text { Boundary Conditions }}$}

12 Six different boundary conditions were proposed for this experiment. The slabs

13 prepared to evaluate the first five conditions mentioned in section 7.1 were prepared and

14 placed in an outdoor exposure site in West Lafayette, Indiana at the INDOT research

15 facility. The samples were exposed to the environment from November 8, 2009 to May

161,2010 . During this time the slabs were exposed to fall, winter, and spring conditions.

17 The weather conditions were monitored by wireless weather station positioned near the

18 samples. The samples were placed outside after approximately 48 days of curing at $23 \pm$

$191{ }^{\circ} \mathrm{C}$ and $98 \pm 2 \%$ relative humidity. The sixth sample was placed in a constant

20 temperature $\left(23 \pm 0.2{ }^{\circ} \mathrm{C}\right)$ and relative humidity $(50 \pm 2 \%)$ environmental chamber for an

21 equivalent exposure time as a control sample. This is shown in Figure 8.1. 


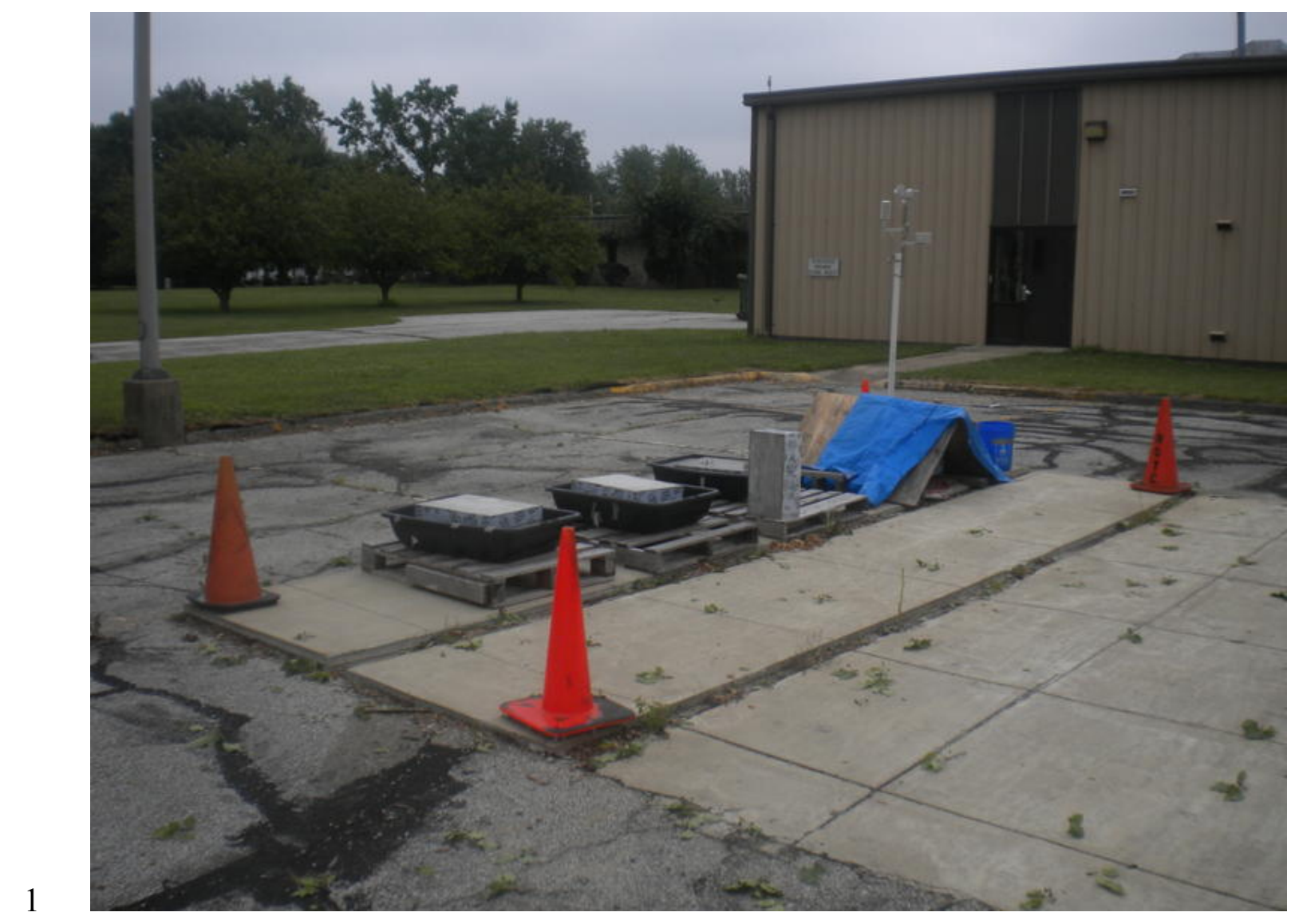

Figure 8.1: Exposure Samples at the INDOT Research Test Facility

Several attempts were made to obtain an agreement from the weather department

4 of the television station located near the INDOT research office. While it was initially

5 thought that this would be able to be done, the television stations unresponsiveness

6 caused the research team to install a weather station. Figure 8.2 shows the weather

7 station that was used in this study that was alongside the slabs being tested.

8 The first sample (a covered surface) was covered at the surface from direct

9 contact with surface moisture a tent like structure made out of two pieces of plywood,

10 and can be seen in Figure 8.3. Wood planks were used to raise the slab off the ground so 
1 any pooled water would not be in contact with the specimen. This boundary condition

2 would represent a concrete that is protected from the direct precipitation.

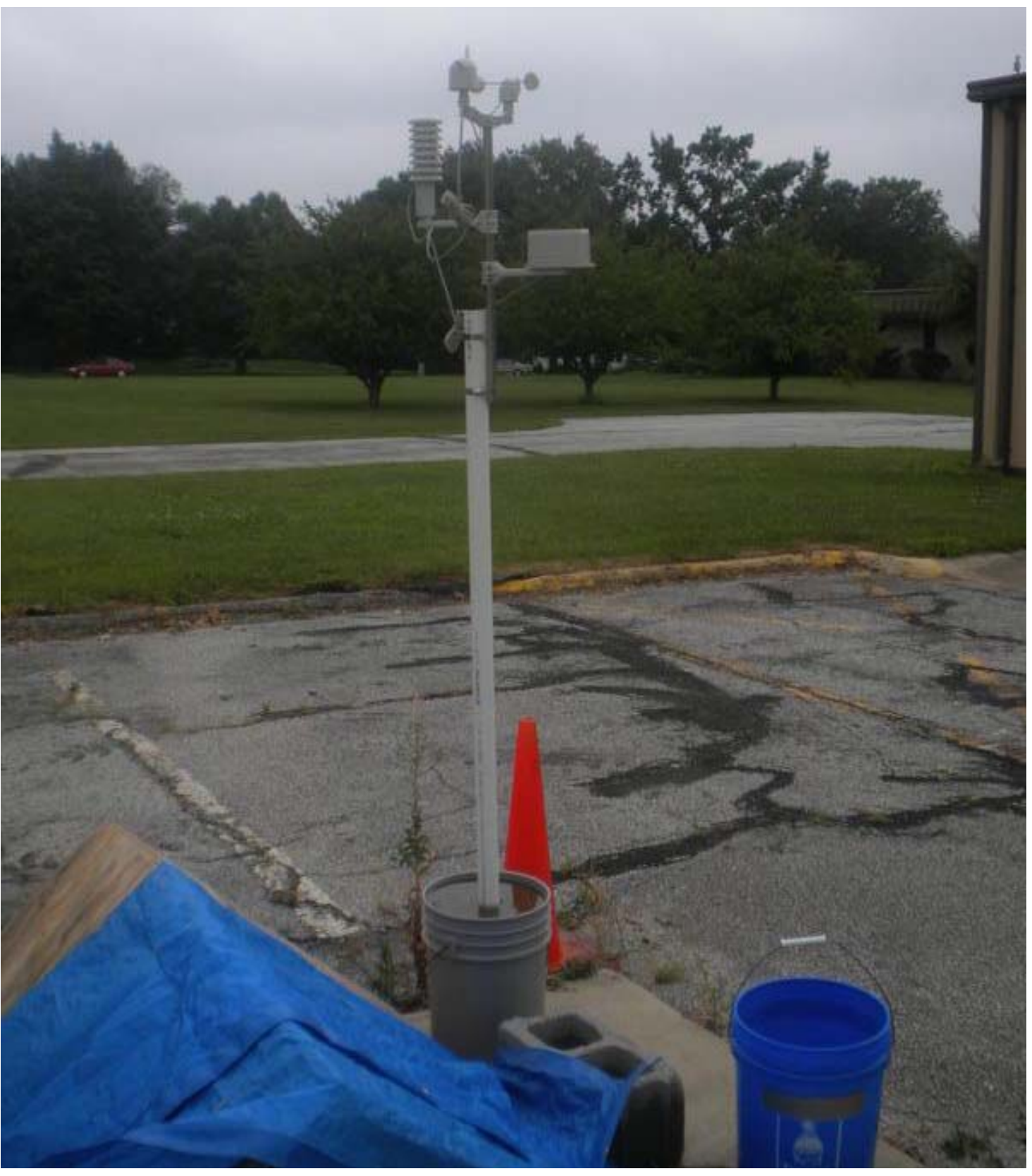

Figure 8.2: Weather Station at the INDOT Research Test Facility 


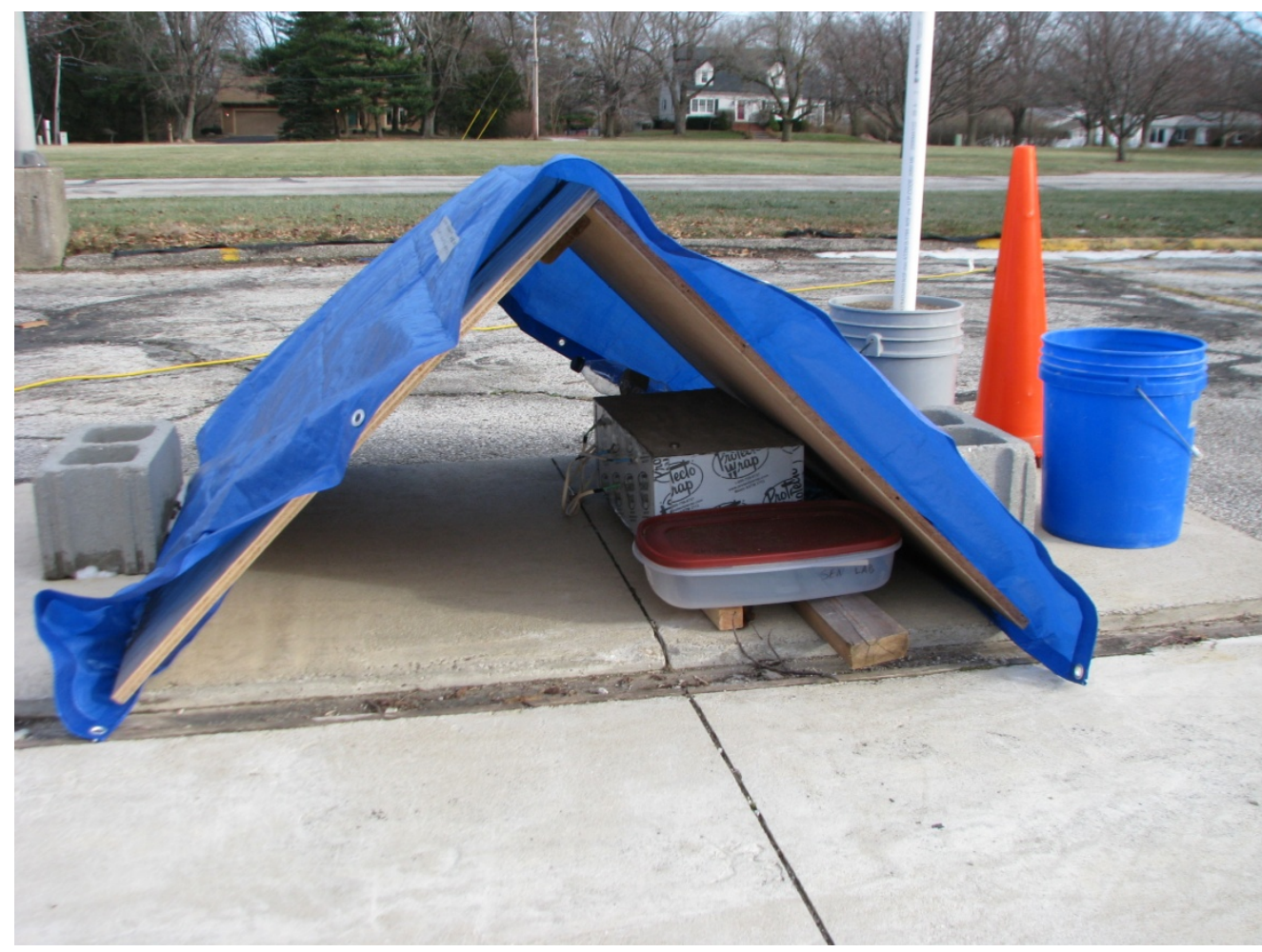

Figure 8.3: Covered Sample at the INDOT Research Test Facility

3 The second sample tested was the exposed vertical surface. To create this

4 boundary condition, a slab was placed on its end so the exposed surface was

5 perpendicular to the ground as shown in Figure 8.4. The slab was placed on a wooden

6 pallet so any pooled water would not be in contact with the specimen. This boundary

7 condition represents a concrete capable of shedding water like an exposed concrete

8 bridge column that may or may not always be directly contacted by precipitation

9 depending on the prevailing winds. 


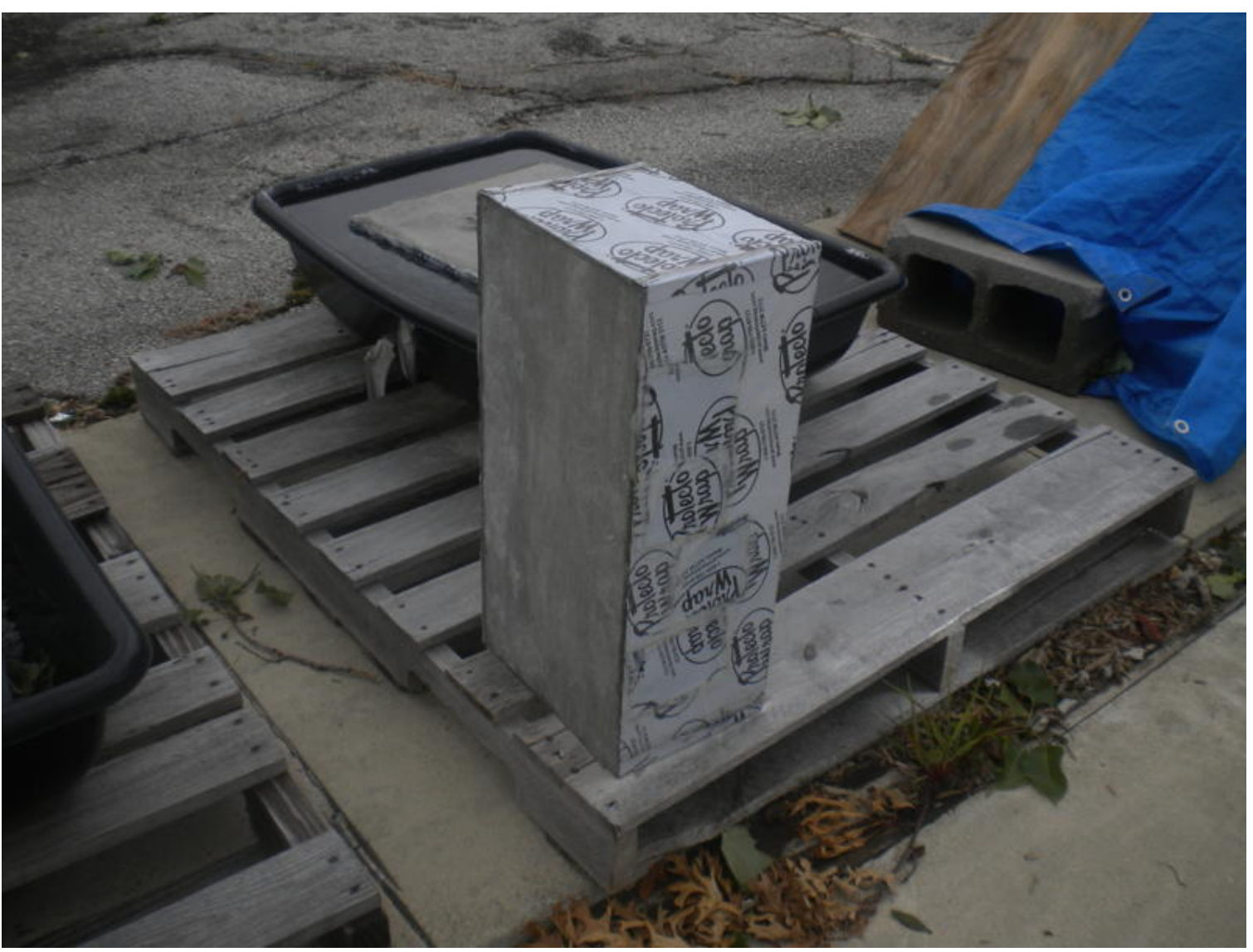

Figure 8.4: Exposed Vertical Surface Sample at the INDOT Research Test Facility

The third and fourth boundary conditions were designed to evaluate the conditions

4 of the base the concrete is placed on different subgrades (Figure 8.5 and Figure 8.6). The

5 third sample was placed on a drainable base while the fourth sample was placed on a non-

6 drainable based. These base conditions were created using a plastic tub with open graded

7 aggregate lining the bottom. The only difference between the third and fourth samples is

8 that the drainable base (sample three) had holes drilled in the bottom of the tub to

9 facilitate the water loss. The non-drainable condition started with a small amount of

10 water in the bottom of a fully intact tub. The non-drainable boundary condition had a 
1 varying water level in the tub according to the precipitation and evaporation. This was

2 designed to represent concrete pavements that may be on different subgrades.

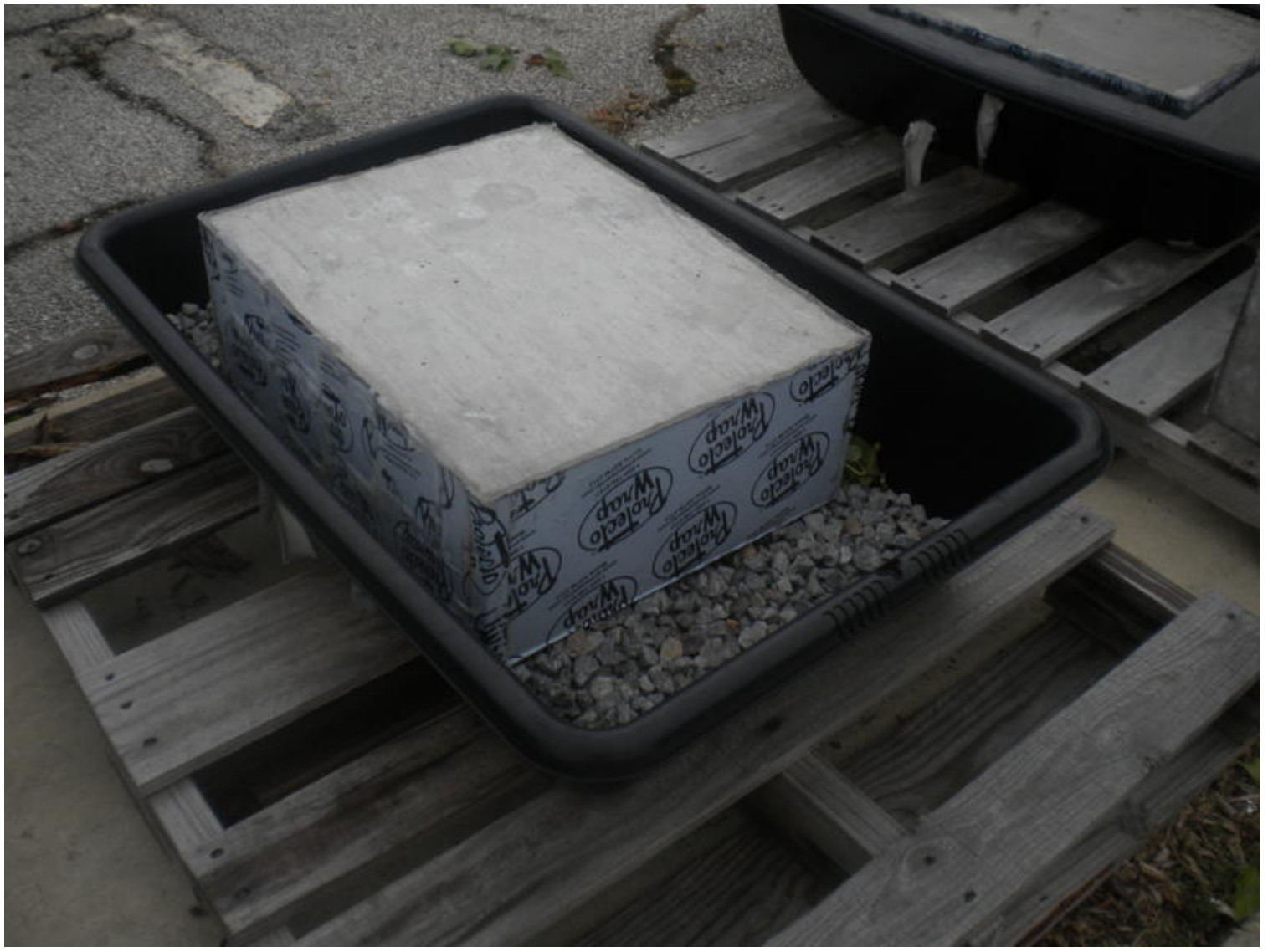

Figure 8.5: A Horizontal Surface on a Drainable Base at the INDOT Research Test Facility 


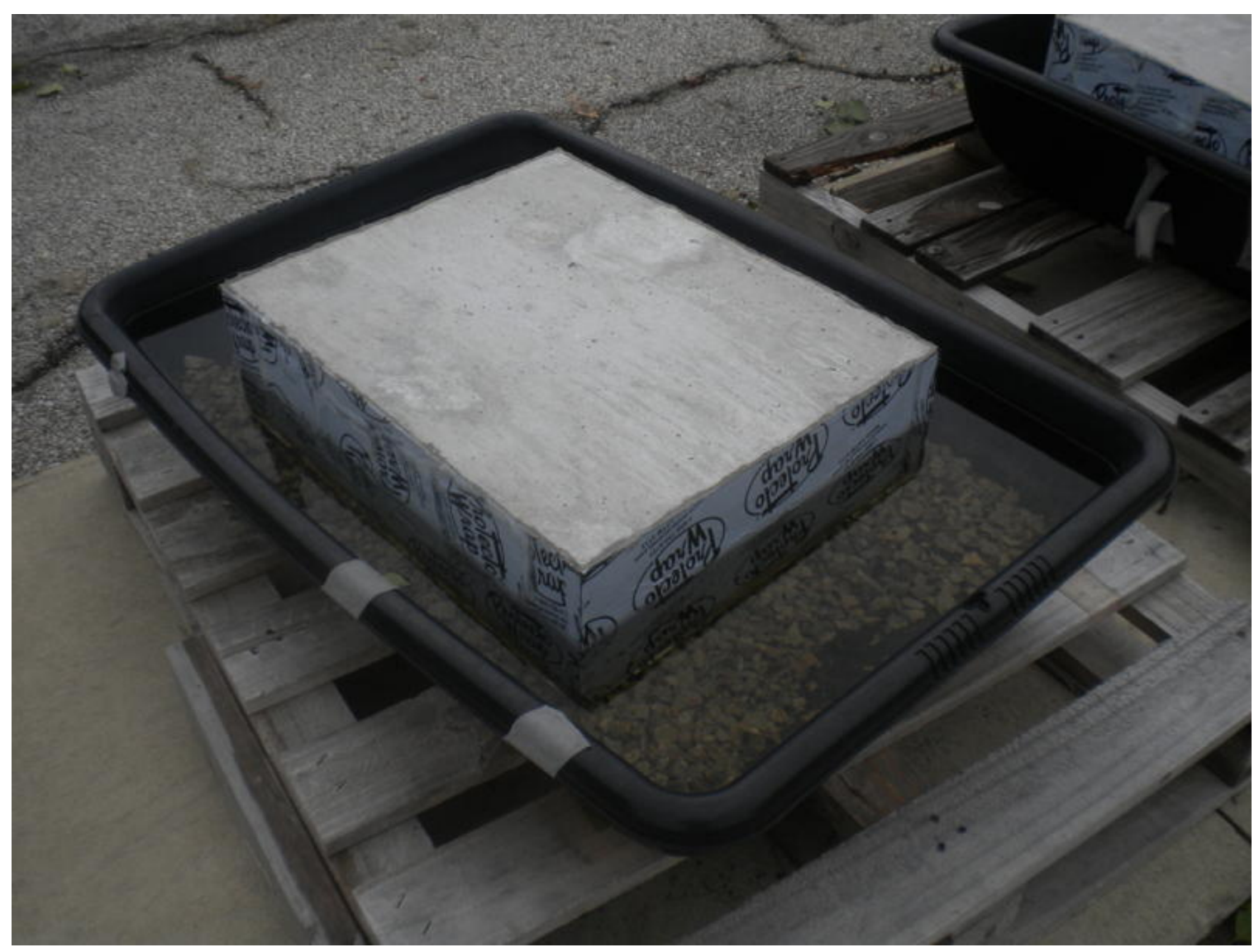

2 Figure 8.6: A Horizontal Surface on a Non-Drainable Base at the INDOT Research Test 3 Facility

The fifth boundary condition consisted of placing a sample was fully submerged

5 from the start of the experiment. This was done by placing the concrete in a plastic bin

6 under water as shown in Figure 8.7. This boundary condition represents a concrete that is

7 in the worst case scenario with respect to developing high internal relative humidity or

8 saturation. 


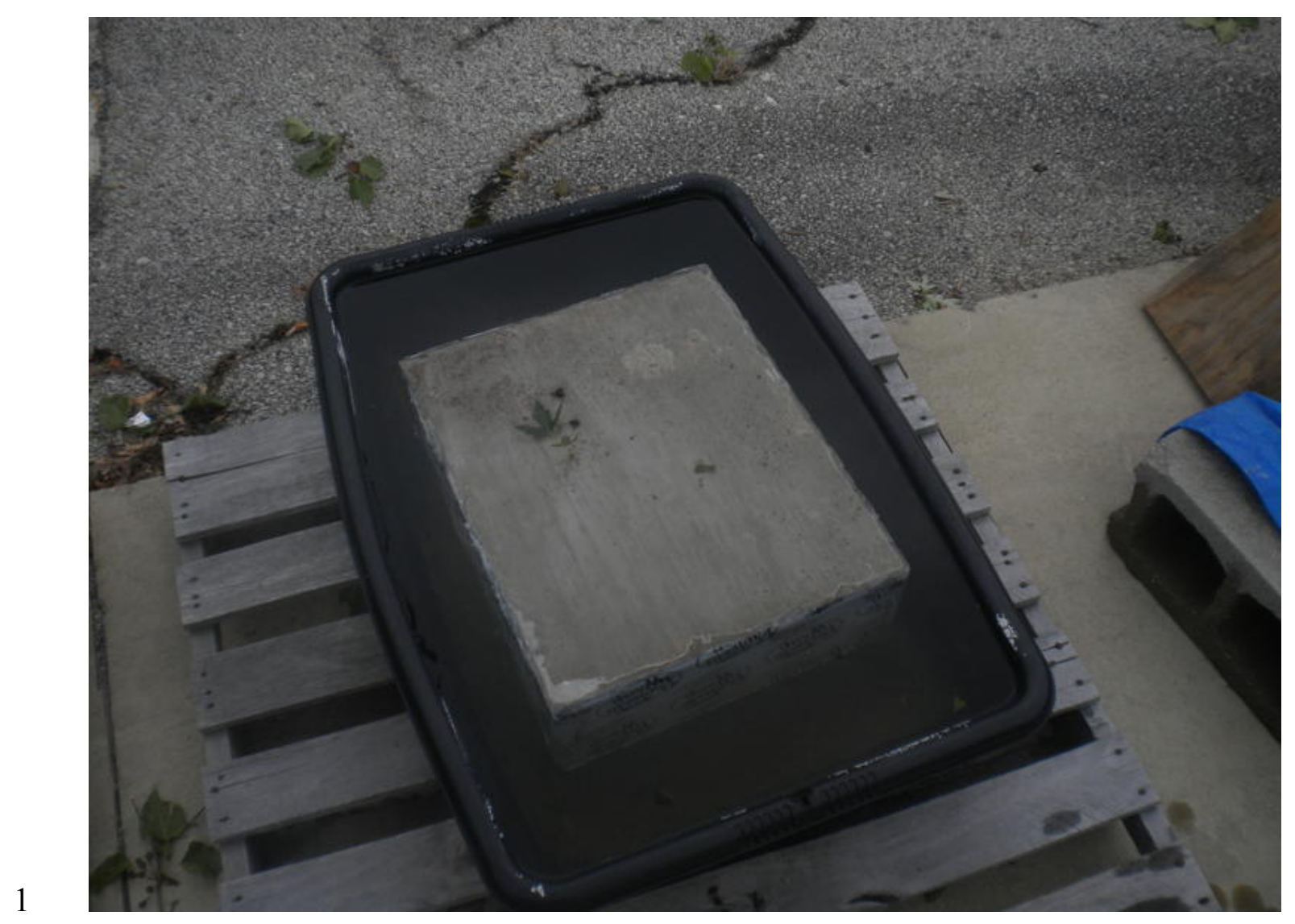

Figure 8.7: A Submerged Concrete Sample at the INDOT Research Test Facility

4 with a constant temperature $\left(23 \pm 1{ }^{\circ} \mathrm{C}\right), \mathrm{RH}(50 \pm 2 \%)$, or precipitation. This sample is

5 shown in Figure 8.8 and represents a sample that may exhibit a high level of drying. 


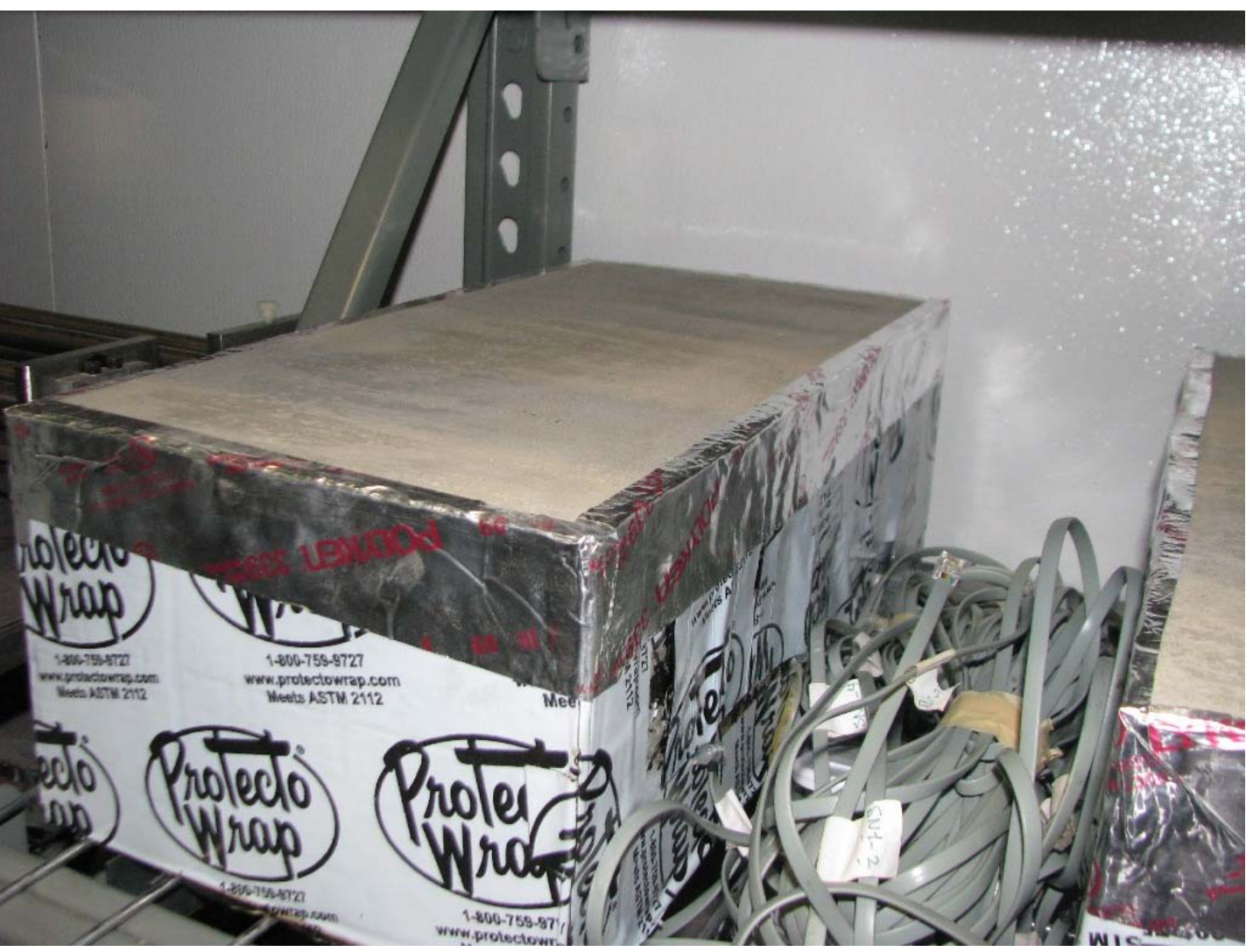

Figure 8.8: A Sample Exposed to a Constant Drying Environment

\section{$\underline{\text { 8.3 Evaluation of the Relative Humidity Sensors }}$}

To test the capabilities of the RH sensors, the sensors were placed above saturated

5 salt solutions according to ASTM E-104. The salt solutions were used to determine

6 whether the relative humidity $(\mathrm{RH})$ sensors could return a stable measurement for higher

7 relative humidities. The salts used to evaluate the sensors were potassium sulfate

8 (K2SO4), potassium chloride $(\mathrm{KCl})$, sodium chloride $(\mathrm{NaCl})$, and potassium iodide $(\mathrm{KI})$.

9 Tables 8.1 and 8.2 provide an illustration for typical salts and relative humidities

10 measured in this program. The humidity created by these salts is temperature dependant;

11 therefore all the salt solutions were maintained at $23 \pm 1{ }^{\circ} \mathrm{C}$. 
Table 8.1: Salt Solution Calibration of Humidity Sensor \#28

\begin{tabular}{|c|c|c|}
\cline { 2 - 3 } \multicolumn{1}{c|}{} & \multicolumn{2}{|c|}{ Humidity (\%) } \\
\hline \multirow{2}{*}{ Salt } & Sensor measurement & Actual RH over saturated salt \\
& 97.3 & solution \\
\hline $\mathrm{K}_{2} \mathrm{SO}_{4}$ & 84.7 & 97.4 \\
\hline $\mathrm{KCl}$ & 75.4 & 84.7 \\
\hline $\mathrm{NaCl}$ & 70.0 & 75.4 \\
\hline $\mathrm{KI}$ & & 69.3 \\
\hline
\end{tabular}

Table 8.2: Salt Solution Calibration of Humidity Sensor \#21

\begin{tabular}{|c|c|c|}
\cline { 2 - 3 } \multicolumn{1}{c|}{} & \multicolumn{2}{c|}{ Humidity (\%) } \\
\hline \multirow{2}{*}{ Salt } & Sensor measurement & Actual RH over saturated salt \\
& & solution \\
\hline $\mathrm{K}_{2} \mathrm{SO}_{4}$ & 96.7 & 97.4 \\
\hline $\mathrm{KCl}$ & 84.4 & 84.7 \\
\hline $\mathrm{NaCl}$ & 75.0 & 75.4 \\
\hline $\mathrm{KI}$ & 70.1 & 69.3 \\
\hline
\end{tabular}

3 


\section{$\underline{\text { 8.4 Selection of a Relative Humidity Sensor }}$}

There are many commercially available relative humidity $(\mathrm{RH})$ sensors. Testing

3 was needed to assess which sample would be the most appropriate for this study

4 considering durability, cost, precision, and accuracy, of the sensors. All sensors have a

5 recommended operating range for both temperature and relative humidity that must be

6 considered. Since the experiment was to be conducted for over a year, outdoors, and in

7 north-central Indiana, a sensor that could function in both freezing and classically warm

8 temperatures was needed. Also, when dealing with a sealed concrete specimen, one must

9 expect the humidity to be relatively high and in an alkaline environment in the sample

10 when the experiment first begins. Typically, as a sensor is subjected to a RH above 80

$11 \%$, the variance and error in the measurement become greater compared to a lower $\mathrm{RH}$

12 range. The following section describes the sensors that were evaluated as a part of this

13 study.

15 These relative humidity sensors cost approximately $\$ 20-\$ 25$. These sensors

16 (HIH-3610, HIH 4000) needed to be coupled with some type of temperature probe

17 running simultaneously with the $\mathrm{RH}$ sensor. The sensors were a capacitive type of

18 sensor. The RH sensor works by measuring a voltage, which, when coupled with the

19 accompanying temperature, can be used to ascertain a relative humidity measurement.

20 The sensors were soldered to standard copper wires and need to be continually connected

21 to data logging equipment. Since the concrete slabs had to be placed outside, there

22 would need to be shelter nearby so the cords run outside the building with the computers 
1 safely inside. When testing the environmental chamber type RH sensor over the

2 saturated salt solutions, the sensor read a maximum value of approximately $80 \% \mathrm{RH}$,

3 even though the sensor should have returned a reading over $97 \%$. This pointed out that

4 substantial corrections would be needed to make these sensors work in the range if this

5 was ever possible. Since the internal relative humidity for a sealed concrete system would

6 be expected to be between $80 \%$ and $100 \%$ and the sensors would require separate data

7 acquisition this sensor was not a good candidate for this research program.

\subsubsection{I button Sensors}

9 A commercial sensor called the I-button ${ }^{\circledR}$ produced by Maxim Products was

10 evaluated as a potential candidate for this research. The I-button is a single device that

11 measures both relative humidity and temperature. The device is a stand alone device

12 which means that the device will log data without being attached to any data logging

13 device. Because of the I-buttons internal battery and onboard memory, the device can

14 hold over 4000 data points (the data collection rate can be adjusted during the sensor set-

15 up) and that can be downloaded at any time. Because of the I-button's stand alone

16 capability, this introduced the possibility of easily placing the slabs outdoors to get real

17 weather exposure conditions.

18 The size of the I-button made it capable of being placed in a sample, though an

19 even smaller sensor would be optimal. Although, one specific limitation of the I-button's

20 set up needed to be overcome. As explained before, the I-button is a stand alone device,

21 meaning it will acquire data without being attached to a data acquisition system. 
The primary method for using the I-button was directly connecting an I-button to

2 a reader provided by the manufacturer. The reader consists of a molded plastic head with

3 electrical contacts inside to connect with the I-button. The other end of the cable is a

4 USB connection that enables the data to be downloaded to a computer. The I-button is

5 placed inside this head and can data can be obtained at any point during the acquisition.

6 The need to monitor the progress of the experiment without removing the sensors

7 from the slab was desired. Toward this end the direct connection to the I-button was

8 needed so that the sensor could be read while the experiment was in progress. As can be

9 seen in Figure 8.9, the size of the I-button by itself is approximately the size of a quarter

10 in diameter. If the I-button were connected with the reader, it would roughly double the

11 size of the I-button, negating any advantage gained by the small size of the I-button. To

12 get around using the oversized reader for direct connection, a procedure was created to

13 manufacture a custom "reader". A phone wire was connected to contacts located on the

14 top and bottom face of the I-button, respectively to enable the phone wire to protrude

15 from the specimen. 


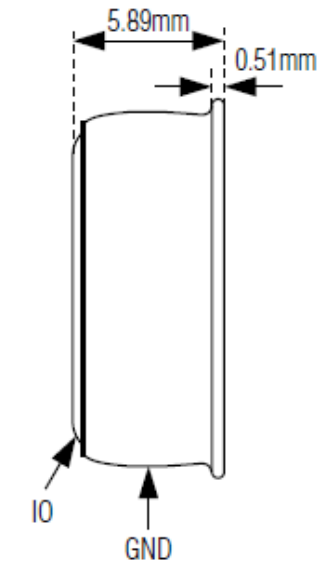

1

$497 \% \mathrm{RH}$ mark with an acceptable amount of variance. It should however be noted that

5 when the sensors were placed in fresh concrete there appeared to be a reaction with the

6 alkaline pore solution which caused several of the sensors to malfunction. One problem

7 with simply placing a sensor directly into concrete is the aggressive nature of fresh

8 concrete. With its highly basic chemistry, fresh paste can damage sensitive electronics

9 that come in contact with it. To overcome this limitation the sensor was not placed in

10 contact with the fresh concrete, but instead was placed in a cavity inside the concrete

11 after it hardened.. The cost of the sensor was between 60 and 100 dollars depending on

12 the quantity ordered. 


\section{$\underline{\text { 8.5 Design of the Slab Specimens }}$}

Due to the problems that were observed with placing the sensors in direct contact

3 with the pore solution, a decision was made to place cylindrical cavities in the sample to

4 monitor relative humidity (Parrot 1982, Grasley 2006). To obtain a relative humidity

5 profile throughout the depth of the sample several sensor locations were used. It was

6 decided early on in the research that the sample geometry should be a slab with one- or

7 two sided exposure to the environment. This was for ease of construction, handling, and

8 analyzing of the obtained data.

9 Many different depth configurations and designs were considered for this slab. It

10 was originally envisioned that an object would be cast in the concrete which could be

11 removed after set thereby creating a cavity in the concrete. The samples would then be

12 sealed and cured. When the experiment was set to begin, the sensors would be placed in

13 the cavity left in the sample and the ends of the cavity could be filled with some type of

14 material to prevent moisture flow through the ends of the cavity.

15 The type of material for which to fill the cavity was also a major point of

16 discussion. In order to get a good relative humidity measurement from the sensors, the

17 cavity in which the sensors rest must come to into equilibrium with the surrounding

18 concrete system. The original hypothesis was, the faster the equilibrium process, the

19 more reflective the measurement would be of the actual sample's state. It was also

20 logically assumed that a smaller cavity would come into equilibrium faster with the

21 surrounding system faster than a larger one.

22 Using this logic direction of thoughts, it was suggested to place some type of

23 cementitious material inside the sample cavity. A cementitious material would mimic the 
1 thermal and diffusive properties of the concrete from which the sample itself is

2 composed. However, there was a desired to keep the sensor away from the paste while it

3 was in the fresh state. Another problem with using a cementitious material to fill the

4 cavity was that it would add water to the sample. An alternate solution needed to be

5 explored. An advantage to not using cementitious filler is there is no permanent block on

6 the cavity, restricting access to the sensor if it somehow malfunctions during the test. A

$7 \quad$ sensor can easily be changed out without having to destroy the test specimen.

8 The wetting and drying occurs all in the vertical direction. As such the cavities

9 should not be placed in line with each other along the vertical direction. This means that

10 all sensors must have a horizontal offset from one another to avoid possible influence

11 from the other cavities. The sensors also need to be placed a sufficient distance from the

12 sample ends to avoid edge effects. As a result, the first sensor was placed at least half the

13 depth of the sample away from any boundary of the sample.

14 The creation of a cavity, or "tunnel" that runs completely through the sample

15 made the construction of the slab easier. It was also easier to ensure that the cavity was

16 placed at the correct location.

17 The final slab dimensions can be seen in Figure 8.10. The form was made using

182 " $x 8$ " (nominal dimensions) lumber as the sides of the mold, and plywood as the bottom

19 of the mold. The center of the cavity that is used for measuring relative humidity was as

20 follows: $0.5 ", 1$ ", 2", and 3.625" from the top surface. The samples were cast upside

21 down to have a consistent surface finish. 


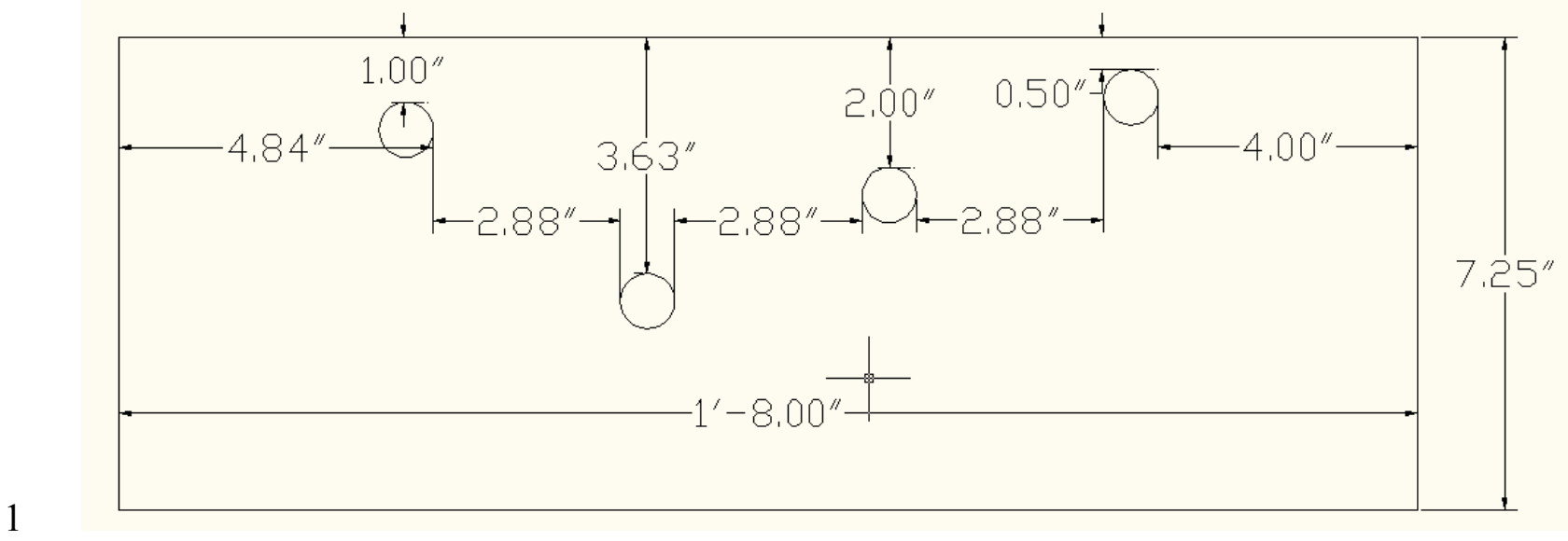

Figure 8.10: Test Slab Dimensions

The mold was prepared with the sides of the mold being covered with plastic

4 sheeting to ease the demolding process. In ASTM tests where concrete surface integrity is

5 needed it is preferred to not use form release agents to stay consistent with these

6 practices. The rectangular steel bars were not covered in form oil. The sample was cast

7 and let sit overnight to set, covered in a plastic tarp. The rectangular steel bar was not

8 able to be removed from the slab without cracking the slabs. Another approach was

9 attempted by first wrapping the steel bars in plastic wrap to help the release of the bars

10 upon demolding. This approach appeared to create a wedging effect that made it more

11 difficult to remove the bars. A similar experience was observed after coating the bar with

12 Teflon tape.

13 It was determined from these trials that the rectangular steel bar were poorly

14 suited for this application. A circular cross section would allow a torque to be applied to

15 break the initial static bond. This twisting action could be continued while a longitudinal 
1 force was applied in conjunction, to remove the piece from the tunnel. A PVC pipe with

2 a $1 / 2$ inch inner diameter fit these characteristics well.

Slabs were cast with the PVC pipe. Ample vibration was applied to make certain

4 material makes it between the PVC pipe and the plywood below. A picture of the casting

$5 \quad$ can be seen in Figure 8.11.

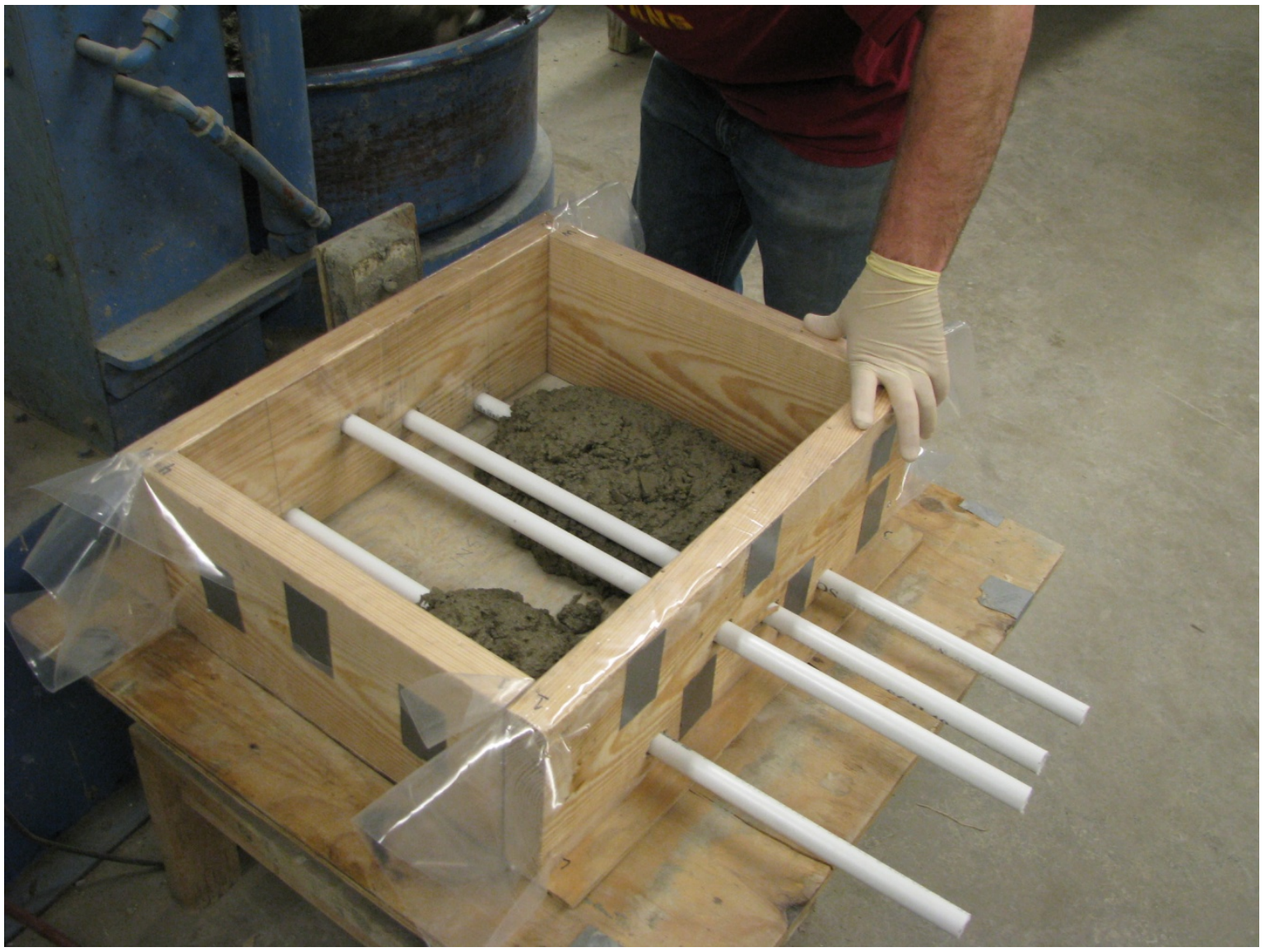

Figure 8.11: Casting of the Slabs

The PVC pipe was removed from the concrete using mechanical means of

9 removal using a tensile force of a Universal Testing Machine (UTM). The slab was 
1 secured to the base of the UTM and clamping the head of the UTM was secured to the

2 PVC pipe as seen in Figure 8.12. The UTM was used to apply a tensile load to the pipes

3 thereby removing the PVC pipes from the concrete.

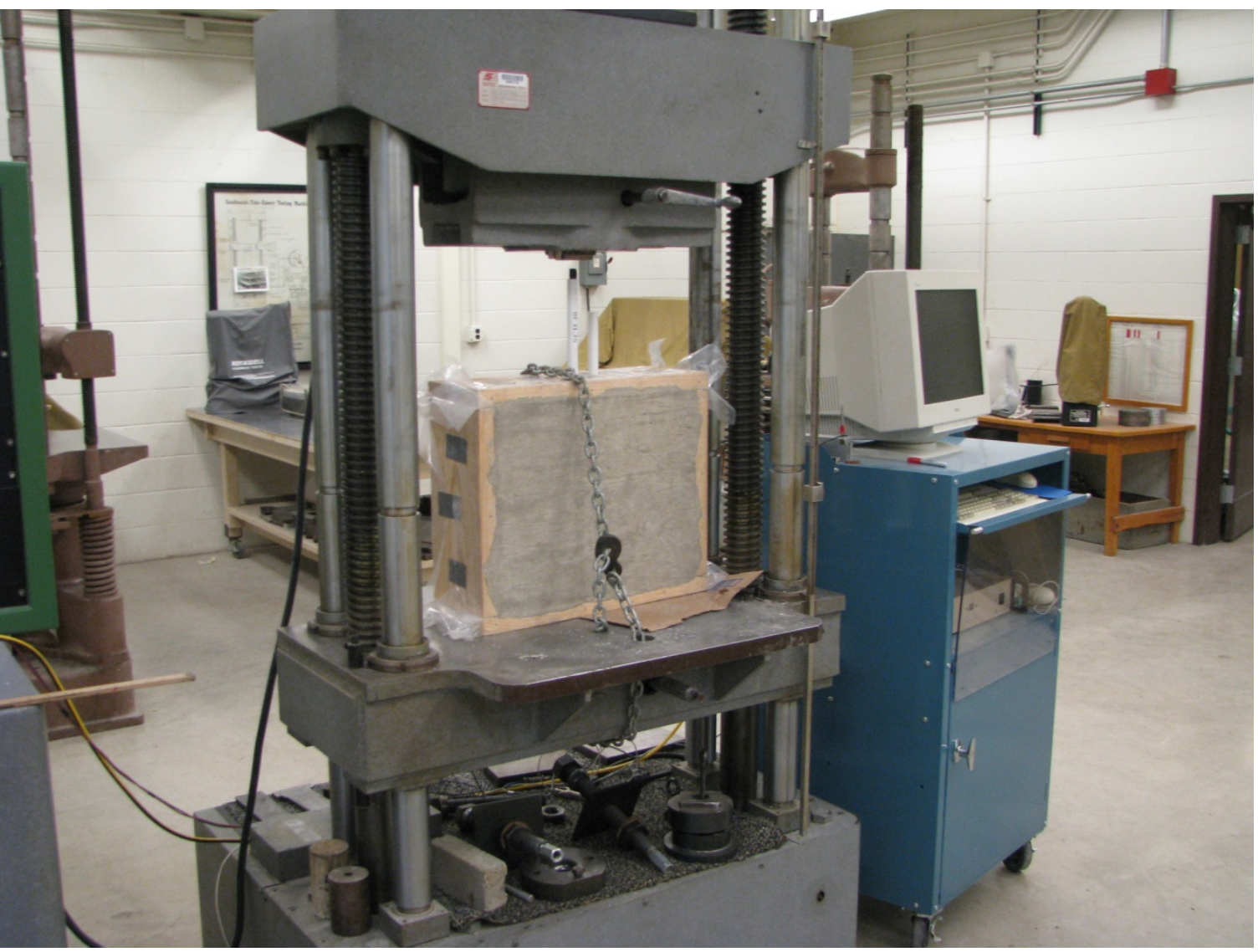

Figure 8.12: Removing PVC Pipes from Slab Using UTM

The samples were sealed and cured for approximately 60 days. Since there were

7 five exposure conditions being tested, a minimum of five slabs needed to be cast;

8 however only two molds used. To help minimize the effect of different ages, the slabs

9 were let cure for a long period of time to let the pore structures reach a point where a few 
1 days maturity would have little effect on the results (i.e., 59 days is similar to 60 days 2 which is similar to 61 days).

4 The I-buttons were attached to phone wires so that they could be placed in the

5 slab. The phone line had a phone line on one end that could be placed in an extension

6 cable that consisted of a phone jack and USB plug for attachment to the computer. The I-

7 button was wired to the free end of the telephone wire as seen in Figure 8.13. 


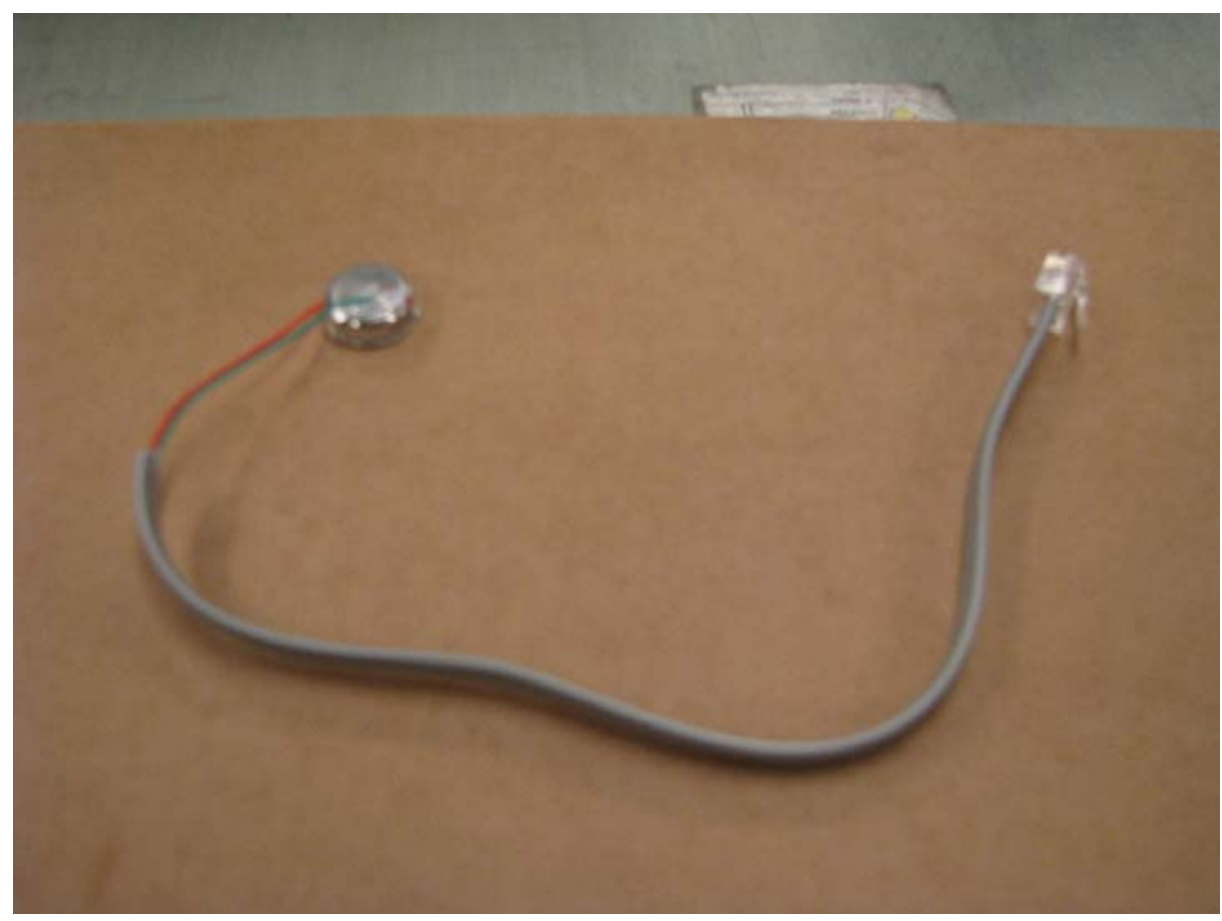

Figure 8.13: I-button Telephone Line Setup

4 soldered to the ends of the telephone wire. To prevent a short circuit from occurring,

5 each splice was adequately insulated with electrical tape shrink tube was placed over the

6 whole connection to help create a more continuous unit for easier handling.

\subsection{Placement of the Relative Humidity Sensor in the Cylindrical Hole}

The sensor was designed to be placed into the slab with a minimal potential for

9 leakage from the ends. They cylinder was prevented from being directly exposed to the

10 open atmosphere. A rubber stopper was used to seal the holes. A small hole was drilled

11 in a \#3 rubber stopper to enable the electrical wires to be fed through the hole. After the 
1 wire was passed through the hole it was injected with pure silicon sealer. After the

2 rubber stopper was placed in the tube end the outside of the rubber stopper was sealed to

3 the concrete interface to create a watertight seal. Since latex sealers release moisture as

4 they cure and silicon sealer hardens by sucking ambient moisture out of the air to cure;

5 both of these sealers could influence the moisture content. The silicon sealer was used on

6 the outside of the specimen since the sealers will cure in the open environment having

$7 \quad$ little effect on the sample results.

8 The electrical wire would typically be soldered to the I-button, however this was

9 not an option with the I-buttons since the housing of the I-button is made of stainless

10 steel. This difficulty arises since soldering stainless steel is difficult because of the

11 chemical composition; the stainless steel must become much hotter compared to mild

12 steel to have solder adhere to it. Further, the I-buttons internal electronics encased inside

13 the small unit, the danger of destroying the I-button with the intense heat was too great.

14 As a result a conductive adhesive was invested as a method to attach the wires. The

15 stainless steel cover displayed poor adhesion properties with the conductive adhesive.

16 The surface can be roughened to increase adhesion, but still does not meet the adhesion

17 of normal steel. To improve the adhesion and electrical properties of solder, a

18 combination of super glue and conductive paint was used. Most adhesives insulate

19 against electrical conduction, making the application of the glue to the wire and I-button

20 difficult. Special care was taken to ensure the super glue was applied to a portion of the

21 wire that still had the insulation attached (Rajabipour 2006). The superglue was left to

22 dry overnight to develop the full strength of the glue. After the glue had cured, the

23 electrical connection was installed from the wire to the I-button. This electrical 
1 connection was made using a silver conductive paint (Pour Ghaz et al. 2010). Before the

2 wires were attached to the I-buttons using super glue, a small piece of wire,

3 approximately one quarter of an inch long, was stripped from the end, exposing the

4 copper strands. With the super glue keeping the insulated part of the wire attached to the

5 I-button housing, the conductive paint could be applied to the area where the copper

6 strands and I-button met, completing the electrical connection and the I-button setup

7 itself.

8 An adaptable and customizable setup is another huge advantage when using the I-

9 button. The main reason a custom setup can be achieved is the I-buttons internal

10 memory. With many relative humidity sensors, a capacitor is used to monitor the

11 ambient humidity in the air. A current is applied across the capacitor and the voltage is

12 monitored by the attached system. This voltage can be converted into a relative

13 humidity. The drawback with this type of sensor is the setup itself must be taken into

14 account when the voltage is output. If too much resistance is intrinsically built into the

15 system, the data output can be incorrect. This is not the case when dealing with the I-

16 button. Since the I-button stores it's data on internal memory and, in turn, relays this

17 memory from the I-button to the user, all that is needed for data transfer is a sufficient

18 electrical connection. No attention needs to be paid to how long the wire is running from

19 the I-button, or how conductive the paint used to complete the connection is; the data is

20 passed by code not voltage output. 
A concrete that was similar to a typical Indiana Department of Transportation

3 (INDOT) paving mixture was used (Table 8.3). The mixture contained entrained air in

4 the range of $5-8 \%$ by volume. As it can be seen from Table 8.4 , the amount of entrained

5 air present in the slab is on the high side of this range at $8 \%$. The mixture had a water to

6 cement ratio of 0.42 with approximately $61 \%$ aggregate by volume. This relatively high

7 paste content made the concrete have a consistent 6" slump using the standard ASTM C-

8143.

Table 8.3: Field Slab Mixture Proportions by Mass in SSD Condition

\begin{tabular}{|c|c|}
\hline Constituent & Mass $\left(\mathrm{kg} / \mathrm{m}^{3}\right)$ \\
\hline Coarse Aggregate (IN \#8) & 944.6 \\
\hline Fine Aggregate (IN \#23) & 786.6 \\
\hline Cement (Type 1) & 464.5 \\
\hline Water (Public Source) & 195.4 \\
\hline Air Entrainer (Micro-Air) & 0.3 \\
\hline
\end{tabular}

10 
Table 8.4: Field Slab Mixture Proportions by Volume

\begin{tabular}{|c|c|}
\hline Constituent & Volume (\%) \\
\hline Coarse Aggregate (IN \#8) & 33.1 \\
\hline Fine Aggregate (IN \#23) & 27.7 \\
\hline Cement (Type 1) & 13.7 \\
\hline Water (Public Source) & 18.1 \\
\hline Air & 8.0 \\
\hline
\end{tabular}

3 Figures 8.14 to 8.17 provide an illustration of the temperature, rainfall, relative

4 humidity, and wind speed measured at the slab locations. It can be seen that during the

5 time of testing the temperature of the air underwent a fluctuation from $30{ }^{\circ} \mathrm{C}$ to $-20{ }^{\circ} \mathrm{C}$. It

6 can be noticed that the temperature swing of approximately $20^{\circ} \mathrm{C}$ is not uncommon. It

7 can be seen that during the time of monitoring there were approximately 30 weather

8 events that resulted in precipitation. While the average relative humidity in the state of

9 Indiana is $65 \%$ the humidity can change rapidly and swings over a wide range. This

10 implies that one can expect substantial drying and wetting hysteresis. It was also

11 observed that a reasonable maximum wind speed for design would be $20 \mathrm{~km} / \mathrm{hr}$ however

12 specific days may exist when the winds gust higher (e.g., during storms). This data will

13 be used to further examine the potential correlations with measured slab behavior. 


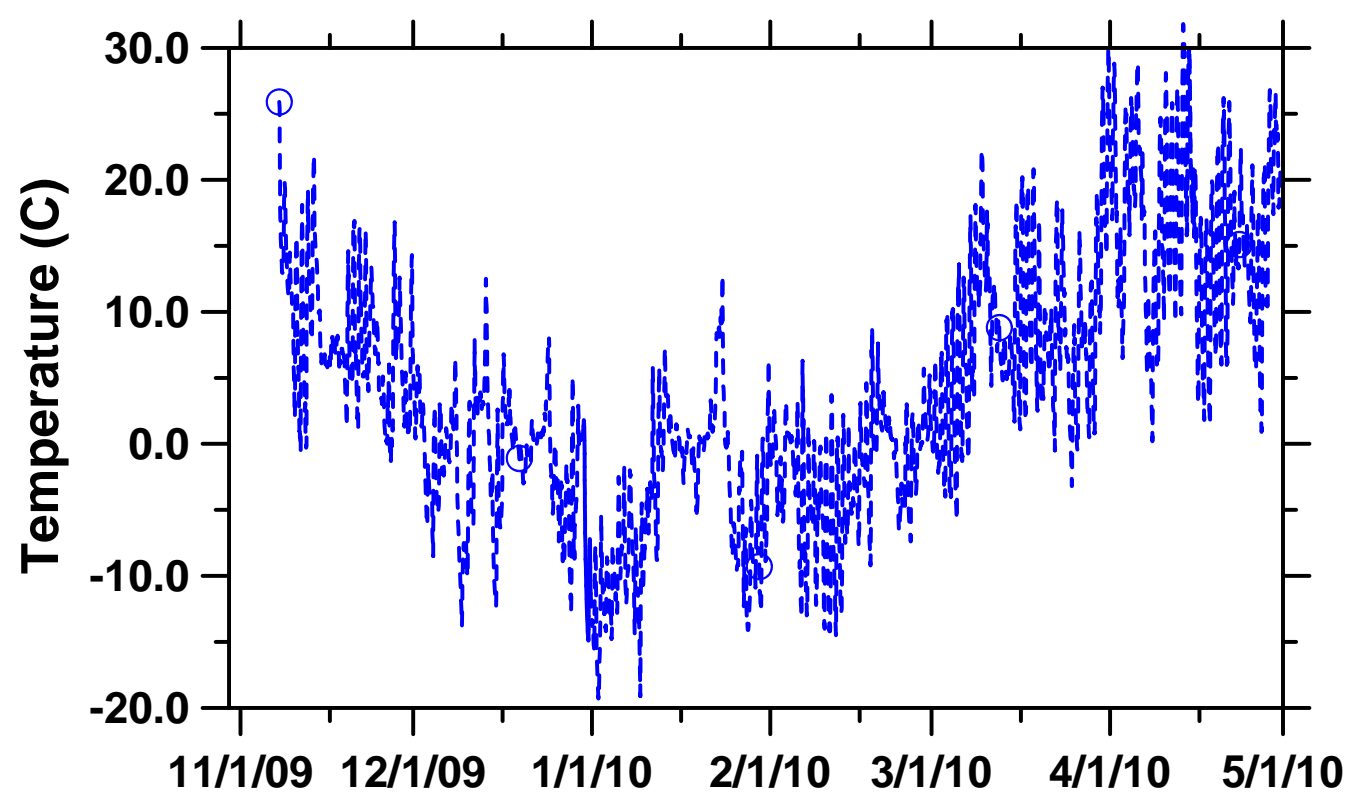

Figure 8.14: Temperature Response from the Weather Station

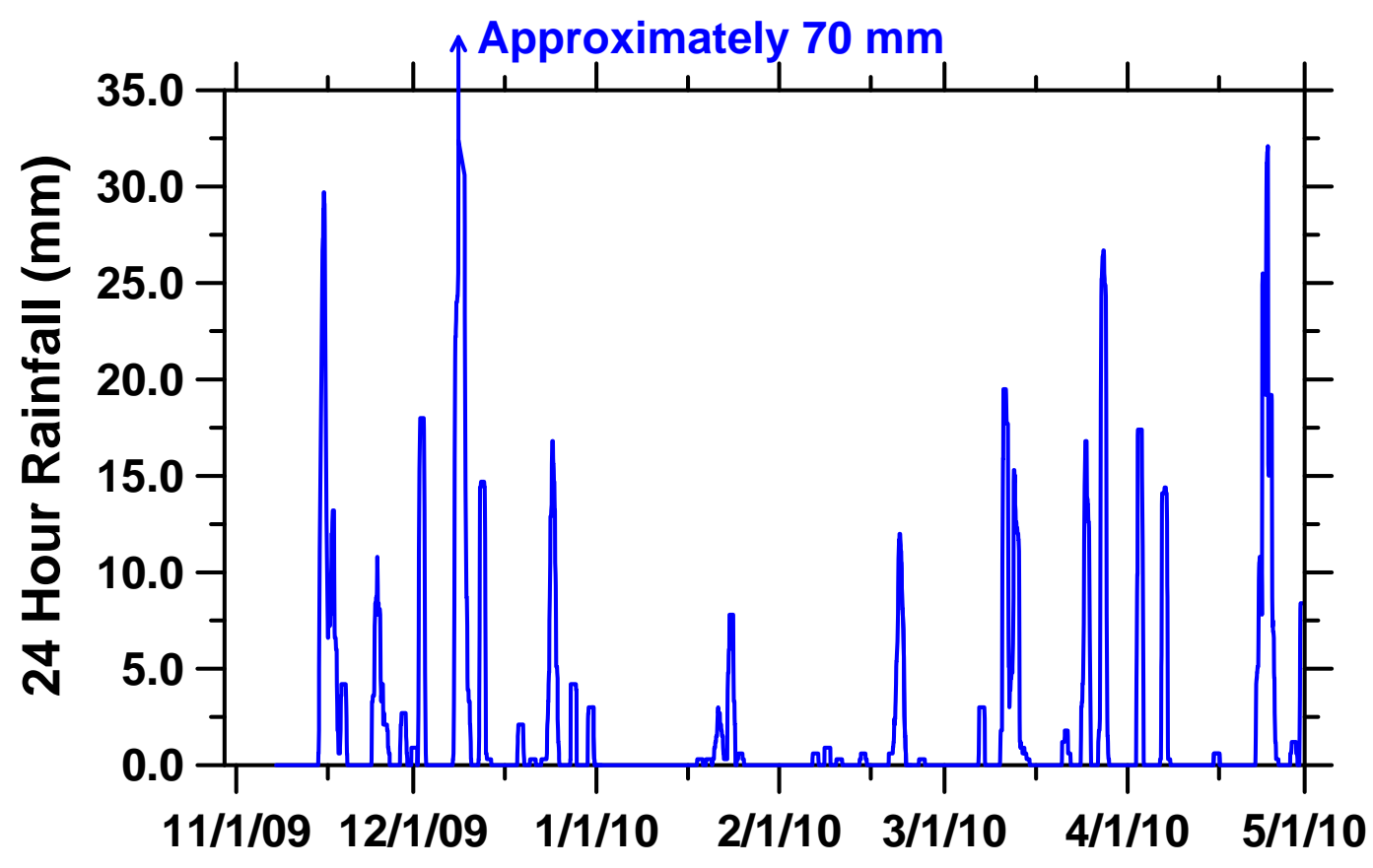

Figure 8.15: Rainfall Response from the Weather Station 


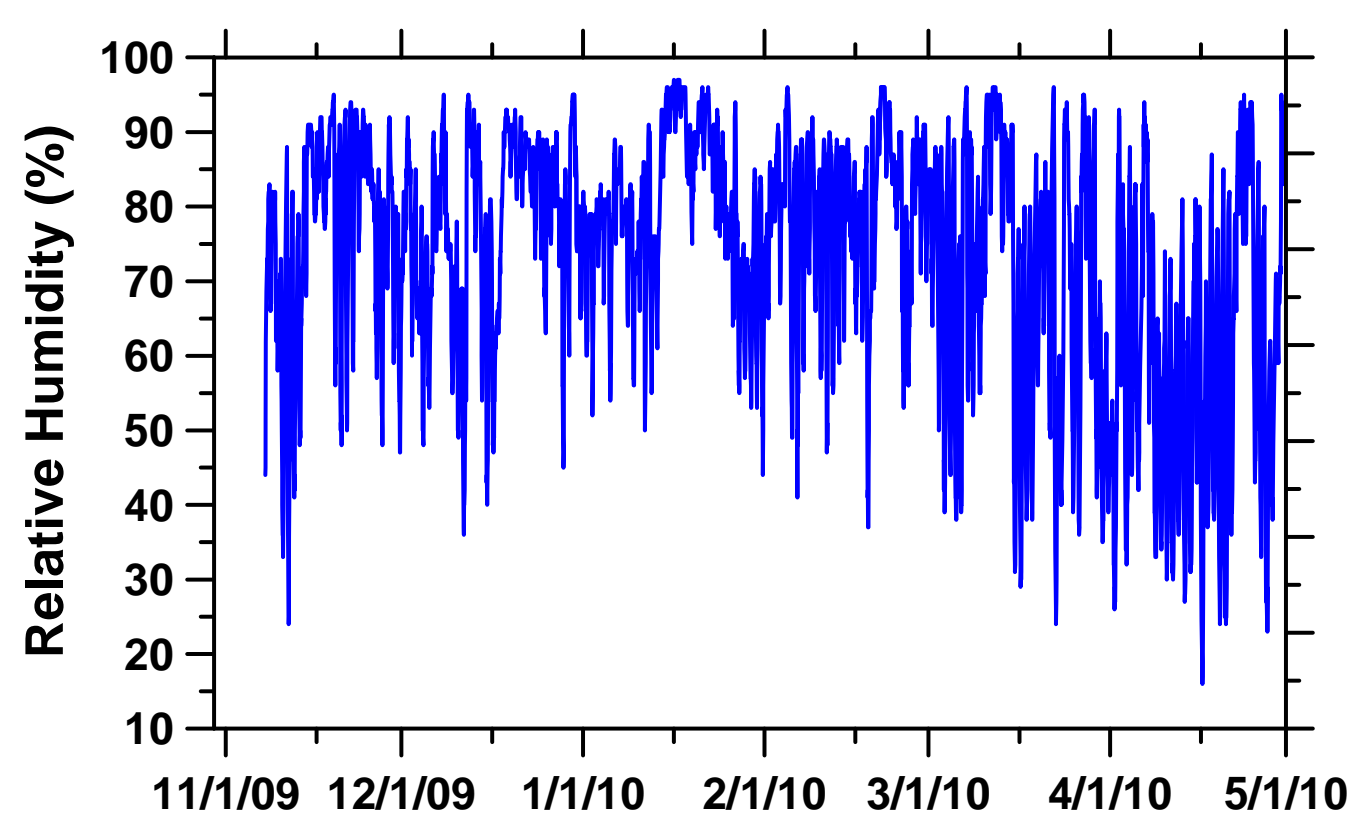

Figure 8.16: Relative Humidity Response from the Weather Station

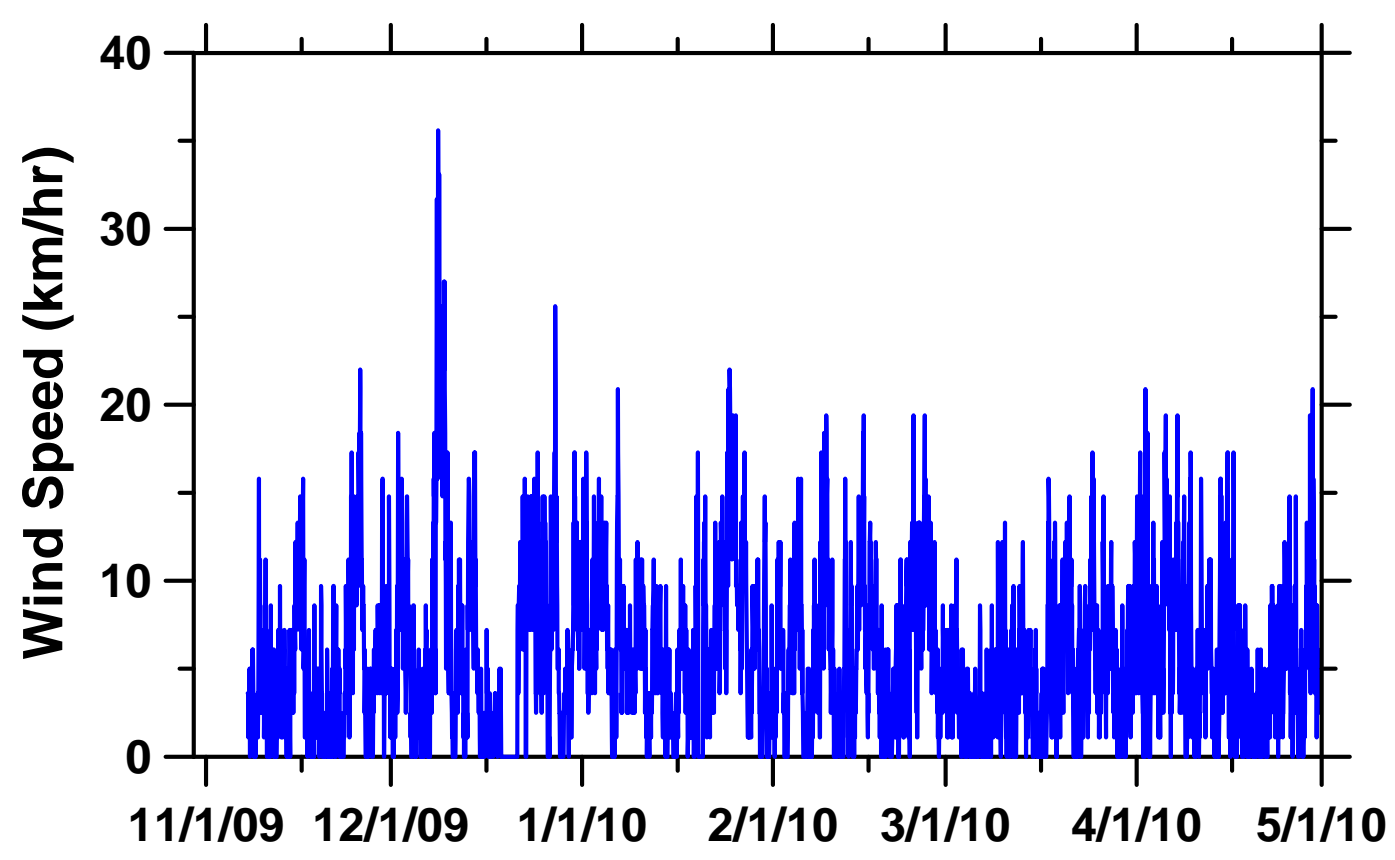

Figure 8.17: Wind Speed from the Weather Station 
1 The following section describes the relative humidity that was measured in the

2 slabs. Two items should be noted however. First, many of the sensors malfunctioned.

3 This points to serious questions with relying on the use of these sensors to provide

4 reliable results when they are placed in a slab for a long time exposure. It is believed that

5 the main reason for the malfunction was related to the potential for water condensation in

6 a high $\mathrm{pH}$ environment around the sensors. In addition, the sensors demonstrated drift

7 over time and needed to be recalibrated. This was done by removing the sensors from the

8 slab and recalibrating them and then reinserting them in the slab. The I-buttons were

9 removed from their cavities inside the slabs and recalibrated over the same saturated salts

10 as referenced earlier. An example of the recalibration can be seen in Table 8.5. A post-

11 experiment calibration correction was applied to all of the data collected in the entire

12 experiment.

13 Table 8.5: Recalibration of an I-button after the Test was Finished (i.e, Post Experiment Calibration Correction)

\begin{tabular}{|c|c|c|}
\hline Salt & Theoretical & Measured \\
\hline $\mathrm{KCl}$ & 84.6 & 91.4 \\
\hline $\mathrm{NaBr}$ & 58.2 & 68 \\
\hline $\mathrm{NaCl}$ & 75.4 & 84.2 \\
\hline
\end{tabular}

15

16 The following section provides results from the humidity sensor measurements

17 taken from the slabs (Figure 8.18 to 8.23 ). It can be observed that even in the slab that

18 was exposed to a constant drying of $50 \%$ relative humidity that the humidity in the core 
1 of the slab remains relatively high dropping to only $75 \% \mathrm{RH} 12 \mathrm{~mm}$ from the surface

2 while the core remains above $90 \% \mathrm{RH}$. The covered slab shows similar behavior with

3 the sensor near the surface remaining at a slightly higher relative humidity (i.e., $80 \%$ )

4 which is consistent with the fact that the average relative humidity in Indiana $(65 \%)$ is

5 higher than the humidity in the chamber (50\%). Similar behavior was observed with the

6 exposed vertical surface however the relative humidity appeared to increase at the end of

7 the test possibly due to rewetting. The remaining slabs consisted of horizontal surfaces

8 placed on a drainable or undrainable base or a slab that was submerged. In each of those

9 cases the relative humidity was higher $(>90 \%, 95 \%$ and $100 \% \mathrm{RH})$ than the constant

10 drying, non-exposed vertical surface and exposed vertical surface. As expected the

11 horizontal surfaces are more likely to collect water than the vertical surface causing the

$12 \mathrm{RH}$ to be higher. The sensors in the submerged sample all failed or read slightly higher

13 than $100 \%$ relative humidity suggesting that the concrete was on its way to saturation. It

14 was slightly surprising to see the drainable and undrainable bases providing similar 15 results. 


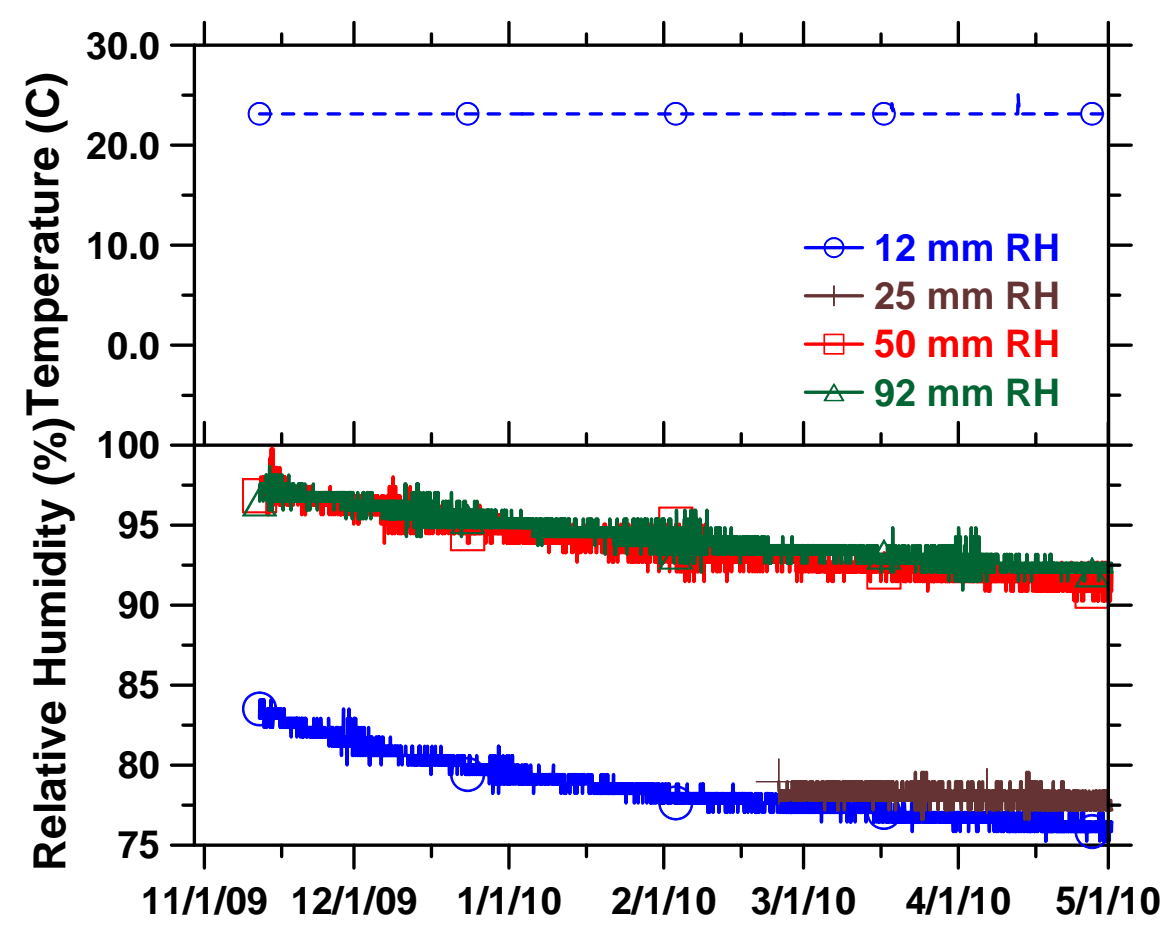

2 Figure 8.18: Relative Humidity Measured in the Concrete in the $50 \%$ Environment

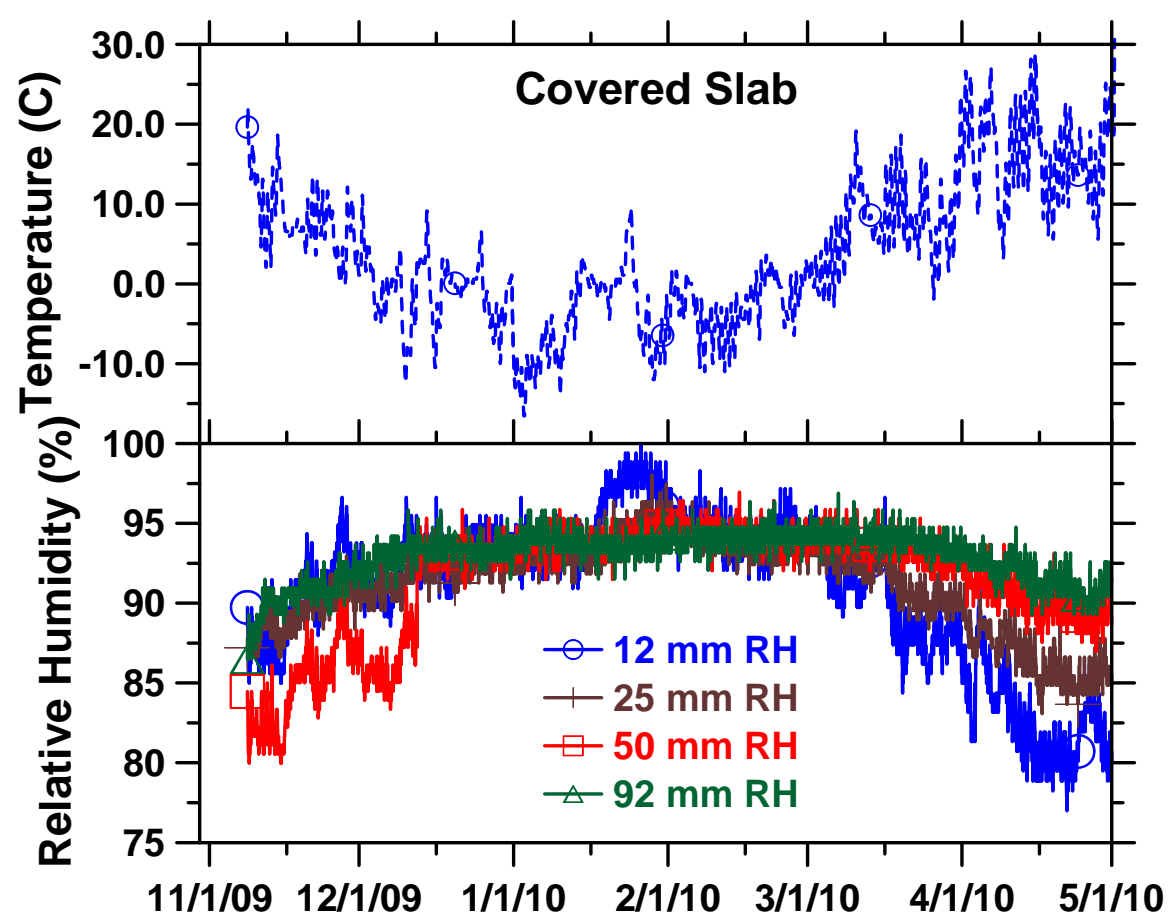

Figure 8.19: Relative Humidity Measured in the Concrete in the Covered Slab 


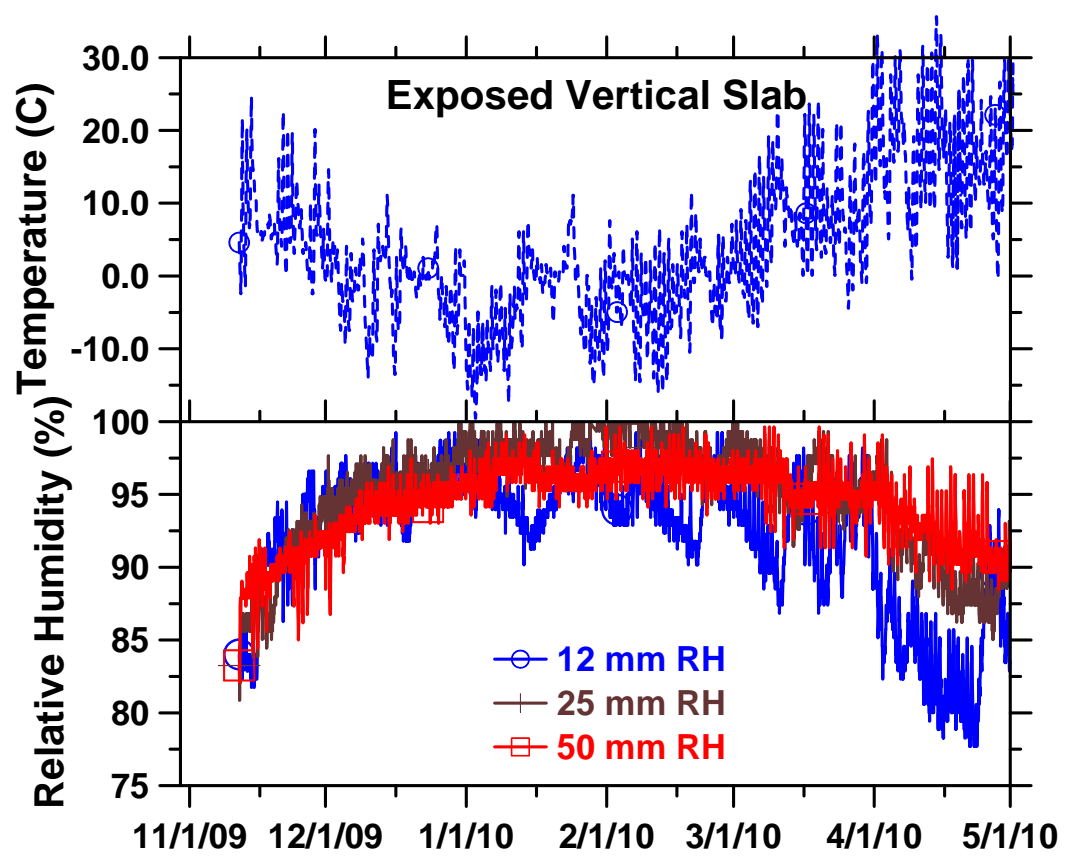

2 Figure 8.20: Relative Humidity Measured in the Concrete in the Exposed Vertical Slab

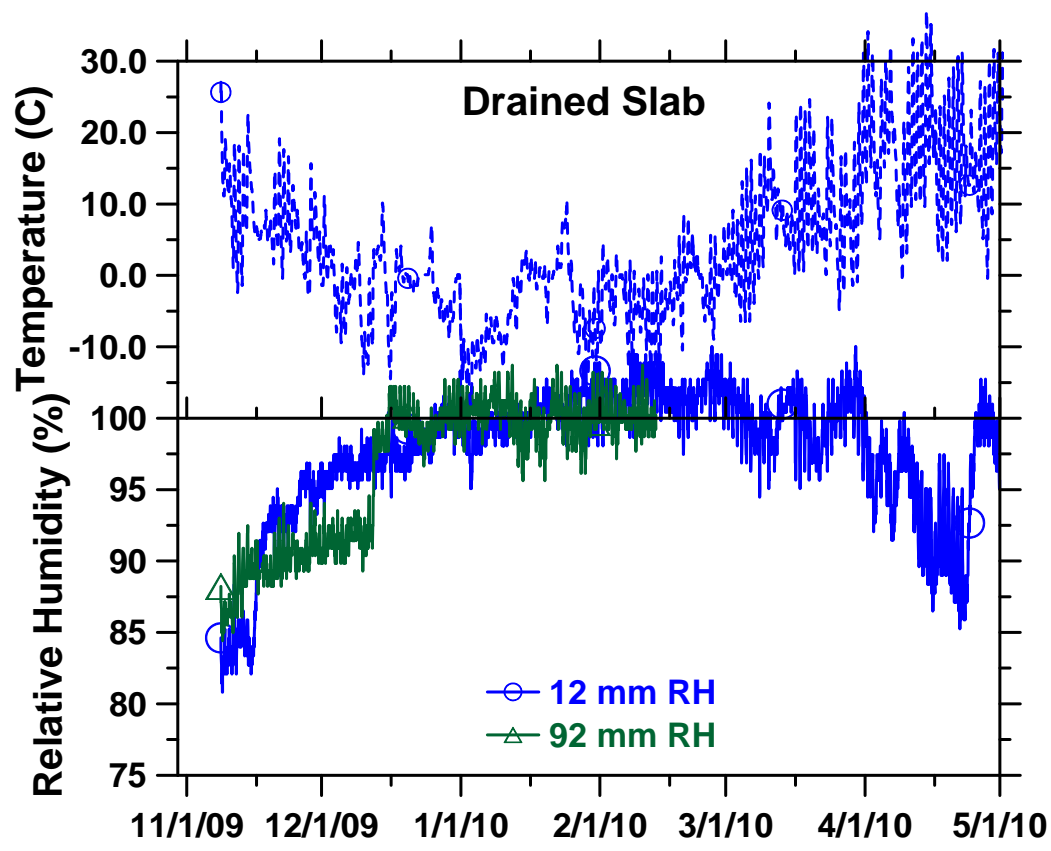

4 Figure 8.21: Relative Humidity in the Concrete in the Slab on a Drainable Base 


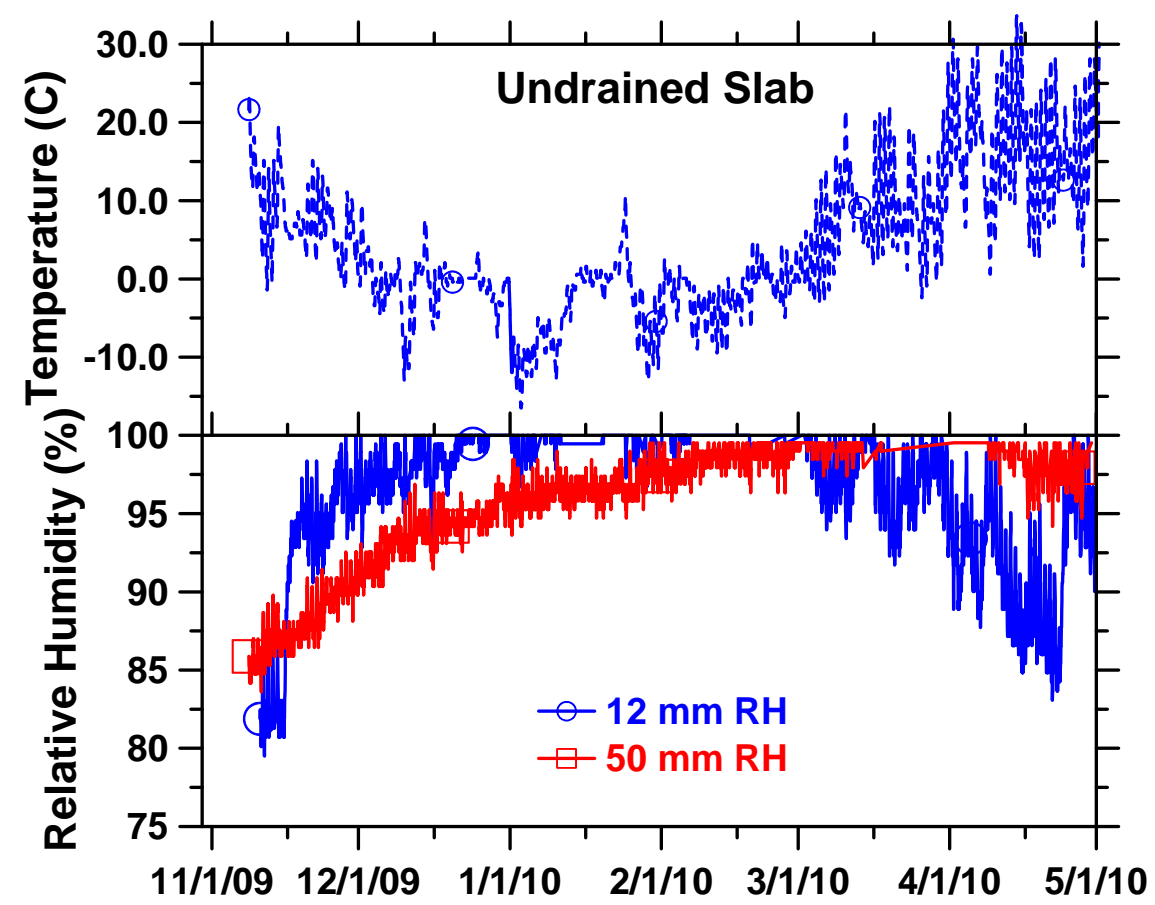

2 Figure 8.22: Relative Humidity in the Concrete in the Slab on an Undrainable Base

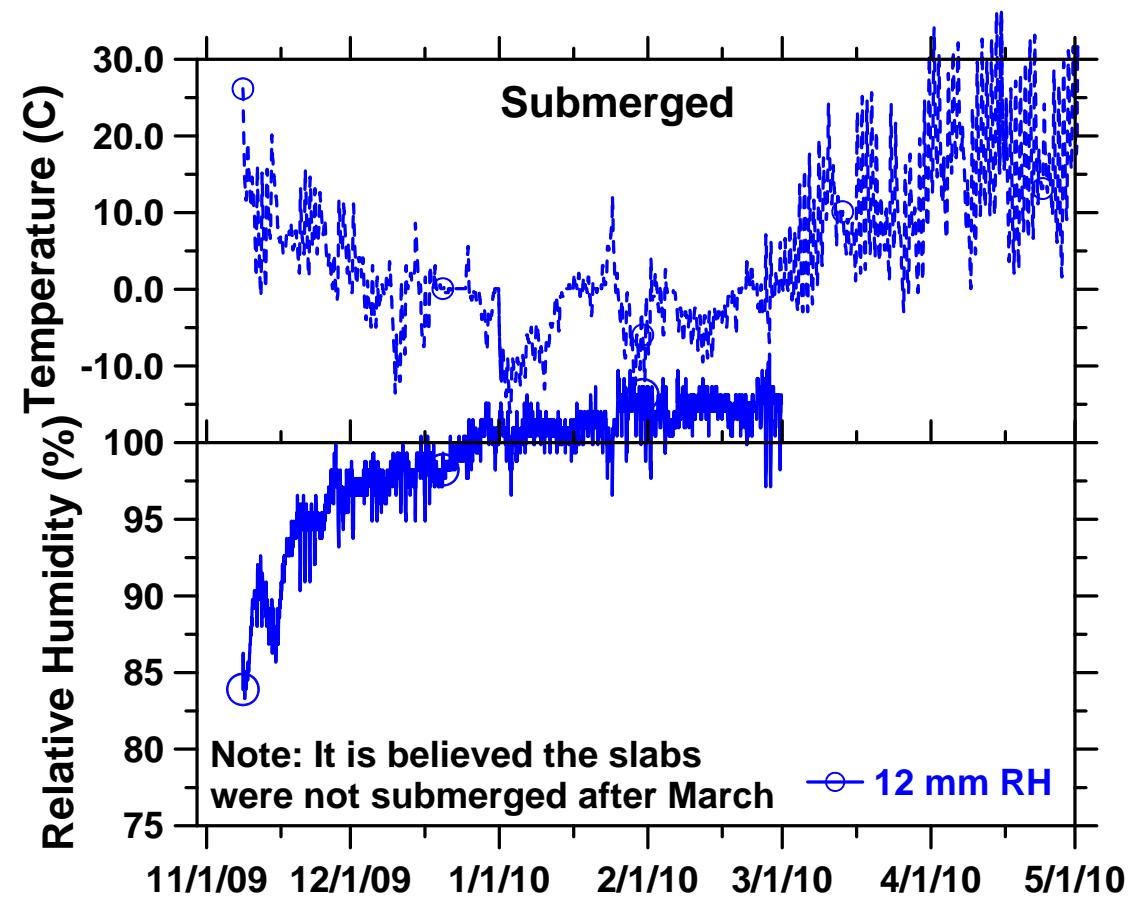

Figure 8.23: Relative Humidity Measured in the Concrete in the Submerged Slab 


\section{$\underline{\text { 8.10 Summary and Conclusions }}$}

This chapter has described the development and testing of concrete samples that

3 were used to monitor field concrete that may be exposed to a variety of environmental

4 conditions. As a part of this study five slabs were placed outdoors and one slab was

5 maintained in an environmental chamber at constant conditions. The slabs tested in this

6 program were used to examine different exposure conditions including: a covered

7 surface, an exposed vertical surface, a horizontal surface on a drainable base, a horizontal

8 surface on a non drainable base, a completely submerged sample and a slab stored at

$923^{\circ} \mathrm{C}$ and $50 \%$ relative humidity. The design of the samples is discussed as well as the

10 evaluation and use of relative humidity sensors. Not surprisingly, the sensors nearest the

11 surface showed the greatest variations in temperature and relative humidity. The main

12 conclusions of this research are that for slabs with a surface protected from direct

13 moisture experienced a relative humidity between $75 \%$ and $100 \%$ while samples that

14 were in direct contact with moisture had a much higher relative humidity. This has

15 implication on the water absorption and diffusion that may be expected in the field. 


\section{CHAPTER 9: SUMMARY, CONCLUSIONS AND RECOMMENDATIONS} 8 testing procedures.

11 of transport properties on concrete pavement in the state of Indiana. This chapter provides

12 an overall summary of the study including the results of an extensive literature review

13 and analysis of the experimental results.

\subsection{Introduction}

This research addressed several issues raised concerning the evaluation of transport properties on concrete pavement in the state of Indiana. This chapter provides a summary of the research study including the results of an extensive literature review and analysis of the experimental results. The results of this study will be used along with results of the Pooled Fund Study that finishes in 2012 to develop and establish new

\subsection{Summary and Conclusions from Experimental Studies}

This report addresses several issues raised concerning the appropriate evaluation 9.2.1 Transport Properties on Concrete Pavement from the State of Indiana The properties of currently used Indiana concrete mixtures were evaluated in terms of water absorption, porosity, and electrical conductivity. A series of concrete paving mixtures were tested to provide a range of values that were typical for the state of 
1 Indiana. While similar mixture proportions were used for the mixtures in Indiana

2 differences in the magnitude of water absorbed occurred.

16 relative humidities: $0 \%$ (oven dry at $105{ }^{\circ} \mathrm{C}$ ), $50 \%, 65 \%$ and $80 \%$. Results show that

17 samples conditioned at a $50 \%$ relative humidity can show a total absorption that is

18 approximately six times greater than similar samples conditioned at $80 \%$ relative

19 humidity. Initial sorptivity, secondary sorptivity and total absorption at 8 days for

20 samples conditioned between $50 \%$ and $80 \%$ relative humidity show a linear trend

21 related to the $\mathrm{w} / \mathrm{c}$ and the relative humidity at which samples were conditioned. However

22 samples that are conditioned by drying in an oven at $105^{\circ} \mathrm{C}$ o not follow the same trend 
1 as samples conditioned in other approaches. This is attributed to two factors: emptying of

2 a wider range of pores, and the potential for microcracking. Therefore, oven drying

3 samples is not recommended as a proper conditioning procedure for this test. Currently

4 work is examining the use of ovens at $50{ }^{\circ} \mathrm{C}$ and different relative humidity and the

5 results will appear in the Pooled Fund Study.

6 Comparing samples containing different volumes of aggregate can also lead to a

7 misunderstanding of the actual absorption behavior. Samples containing higher volumes

8 of cement paste will absorb more water. When the results are normalized by the volume

9 of cement paste, the sample containing lower volumes of cement paste will absorb more

10 water. However, for the materials examined in this study, this difference can be mainly

11 explained by the amount of water absorbed by the aggregates in the sample.

13 This portion of the report describes experimental results from aqueous solution

14 absorption measurements and drying measurements for concrete in the presence of

15 deicing solutions. It was observed that the deicing solutions reduce the rate of fluid

16 absorption. This reduction can be related to the square root of the ratio of surface tension

17 and viscosity of the absorbed solutions. It was also noted that the drying of concrete

18 containing aqueous solutions (with deicers) differs from that of water. The equilibrium

19 relative humidity of the aqueous solution used as absorbent fluid plays an important role

20 on limiting drying.

21 According with these observations, the presence of deicing salts in field samples

22 may considerably impact the fluid absorption when field samples are tested in the lab 
1 using water. This suggests that care must be taken in analyzing field concrete exposed to

2 deicing salt solutions. 14 are similar to those of the ASTM standard test. water are used.

\subsubsection{Automated Water Absorption Test}

ASTM C1585-04 is currently used to determine the rate of water absorption of water by hydraulic cement concretes. While this test is easy to perform, it can be time consuming and can require the timing of data collection to be altered if fluids other than

This study described a procedure which allows for continuous and automated data acquisition. This enables data to be collected at a much more frequent rate and eliminate the influence of the operator. This additional data can provide information for further analysis of the absorption behavior of the samples.

Both the standard and automated procedures were performed on different specimens. The proposed procedure yield reproducible values of rates of absorption that

\subsubsection{Electrical Conductivity on Concrete}

Electrical conductivity tests were performed as a potential method to develop the understanding of rapid test techniques for quality control. This method is directly applicable to concrete in three ways: 1) wenner probe measurements, 2) direct current measurements, and 3) embedded sensors. This study used a modified parallel law to relate the electrical conductivity to the pore volume, pore solution conductivity and the 
1 tortuosity through the pore network. The influence water addition was able to be

2 determined using electrical conductivity. The results also indicated sensitivity to the

3 volume of paste. In addition, the pore solution was observed to be approximately linearly

4 related to the degree of hydration. It was also shown that it is critical that a correction be

5 applied to samples tested at different temperatures. An activation energy of conduction

6 was observed that was approximately $10 \mathrm{~kL} / \mathrm{mol}$ irrespective of water to cement ratio fro

7 the cement tested.

\subsubsection{Effect of Exposure Conditions}

9 In addition to the measurement of transport properties, the relative humidity and

10 temperature were assessed for concrete exposed to different field conditions. The

11 samples considered in this investigation included a sample stored at $50 \%$ relative

12 humidity, covered concrete, a concrete with an exposed vertical surface, a concrete on a

13 drainable base, a concrete on a non-drainable base, and concrete that was submerged in

14 water. The samples showed that for practical field samples the relative humidity in the

15 concrete was always above $80 \%$ for the samples tested. The samples that were exposed

16 to precipitation events demonstrated higher relative humidities and approached $100 \%$.

18 1) If ASTM C1585 is used by INDOT for mixtures that may have dried, it is 19 suggests that the samples be saturated prior to test. 
1 2) If concrete is tested from the field that contains salts, the influence of salts on 2 fluids transport should be considered.

3 3) Electrical methods show promise as a rapid QC/QA technique. They are sensitive $4 \quad$ to $\mathrm{w} / \mathrm{c}$ and paste content.

5 4) The results of this study will be used along with results of the Pooled Fund Study 6 that finishes in 2012 to develop and establish new testing procedures. 


\section{LIST OF REFERENCES}

Abbas, A., Carcasses, M. and Ollivier, J. P. (1999). "Gas Permeability of Concrete in Relation to its Degree of Saturation." Material and Structures 32: 3-8.

Anderberg, A, Wadso, L. (2008), "Method for simultaneaos determination of sorption isotherms and diffusivity of cement-based materials". Cement and Concrete Research, Vol 38, pp: 89-94.

Alhozaimy, A., Soroushian, P. and Mirza, F. (1996). "Effects of curing conditions and age on chloride permeability of fly ash mortar." ACI Material Journal 93(1): 87-95.

Amphora NDT Limited (2009). "Autoclam permeability system" http://www.amphorandt.com/files/products/file/Amphora\%20Leaflet.pdf.

ASTM International, ASTM C305. Standard practice for mechanical mixing of hydraulic cement pastes and mortars of plastic consistency, 2006.

ASTM International ASTM C642. Standard test method for density, absorption, and voids in hardened concrete, 2006.

ASTM International (2004). Standard test method for interfacial of oil against water by the ring method. ASTM International D971.

ASTM International (2009). Standard test method for air content of freshly mixed concrete by the pressure method. ASTM International C231.

ASTM C 1202 (2005), “Standard Test Method for Electrical Indication of Concrete's Ability to Resist Chloride Ion Penetration", ASTM International.

ASTM International (2004). Standard test method for measurement of rate of absorption of water by hydraulic cement concretes. ASTM International C1585.

ASTM International (2006). Standard test method for determining the water absorption of hardened concreted treated with a water repellent coating. ASTM D6489.

Afzai, M., M. Saleem, and M. Tariq Mahmood (1989). Temperature and Concentration Dependence of Viscosity of Aqueous Electrolytes from 20 to 50C. Chlorides of $\mathrm{Na}+$, $\mathrm{K}+, \mathrm{MG} 2+, \mathrm{Ca} 2+, \mathrm{Ba} 2+, \mathrm{SR} 2+\mathrm{Co} 2+, \mathrm{Ni} 2+, \mathrm{Cu} 2+$, and $\mathrm{Cr} 3+$. Journal of Chemical Engineering Data, Vol. 34, pp 339-346. 
Bamforth, P. B. (1987). "The relationship between permeability coefficient for concrete obtained using liquid and gas." Megazine of Concrete Research 38(138): 3-11.

Basheer, P. A. M. (1993). A brief review of methods for measuring the permeation properties of concrete in-situ. Inst. Civ. Eng. Struct. Bldgs.

Basheer, P. A. M. (2001). Permeation analysis, William Andrew Publishing/Noyes Publications.

Basheer, P. A. M., Long, A. E. and Montgomery, F. R. (April 14-16, 1993). The Autoclam Permeability System for Measuring the In-Situ Permeation Properties of Concrete. NDT in Civil Engineering. Proceedings of the British Institute of NonDestructive Testing International Conference, University of Liverpool, UK British Institute of NDT, Northampton.

Barde, V. "Relating Material Properties to Exposure Conditions for Predicting Service Life of Concrete Bridge Decks in Indiana", MS Thesis, Purdue University, 2006

Bazant, Z.P. and Najjar, L.J. (1972), 'Nonlinear water diffusion in nonsaturated concrete,' Mater. Struct., 5 (25): 3-20.

Bazant, Z.P., Ed., Fourth RILEM International symposium on Creep and Shrinkage of Concrete: Mathematical Modeling Northwestern University, 1986.

Bégué P., Gagné R. et Marquis A. (2004) Effects of covercrete quality on the sorption behaviour of concrete, CRIB, Université de Sherbrooke, Sherbrooke, Canada, 21 p.

Bentz DP, Ehlen MA, Ferraris CF, Garboczi EJ. Sorptivity-based service life predictions for concrete pavements. Proceedings. 7th international conference on concrete pavements, Orlando FL, 2001; p. 181-193.

Bentz, D.P., M.A. Peltz, K.A. Snyder, and J.M. Davis (2009). VERDiCT: Viscosity Enhancers Reducing Diffusion in Concrete Technology. Concrete International, Vol. 31, No. 1, pp. 31-36.

Bentz, D. (2000). "Influence of silica fume on diffusivity in cement-based materials II: multi-scale modeling of concrete diffusivity." Cement and Concrete Research 30: 11211129.

Bentz, D., Clifton, J., Ferraris, C. and Garboczi, E. J. (1999). Transport Properties and Durability of Concrete: Literature Review and Research Plan. NIST, Gaithersburg, Maryland. 
Bentz, D. "Influence of internal curing using lightweight aggregates on interfacial transition zone percolation and chloride ingress in mortars." Cement and Concrete Composites Under review.

Bentz, D. P., Quenard, D. A., Baroghel-Bouny, V., Garboczi, E. J. and Jennings, H. M. (1995). "Modelling drying shrinkage of cement paste and mortar Part 1. Structural models from nanometres to millimetres." Materials and Structures 28(8): 450.

Beynerback, R.S. (1983), "Alkali-Silica Reactions in Portland Cement Concrete”, Ph.D. Dissertation, Purdue University, West Lafayette, Indiana.

Bisschop J. Van Mier J. How to study drying shrinkage microcracking in cement-based materials using optical and scanning electron microscopy? Cement Concrete Res 2002; 32(2): 279-287.

Bleszynski, R., Hooton, R. D., Thomas, M. D. and Rogers, C. A. (2002). "Durability of ternary blend concrete with silica fume and blast-furnace slag: Laboratory and outdoor exposure site studies." ACI Materials Journal 99(5): 499-508.

Bockris, J. and Reddy, A. (1970), "Modern Electrochemestry", Vol. 2, Sec. 7.1, Plenun Press, New York.

Boddy, A., Bentz, E., Thomas, M. D. A. and Hooton, R. D. (1999). "An overview and sensitivity study of a multimechanistic chloride transport model." Cement and Concrete Research 29: 827-837.

British Standard Institution (1990). Methods of testing concrete Part 5: Methods of testing hardened concrete for other than strength. BS 1881.

Cabrera, J. (1996). "Deterioration of concrete due to reinforcement steel corrosion." Cement \& Concrete Composite 18: 47-59.

Castro, J., Lura, P., Rajabipour, F., Henkensiefken, R. and Weiss, J., "Internal curing: Discussion of the role of pore solution on relative humidity measurements and desorption of lightweight aggregate", American Concrete Institute, Special Publication 270-8 (2010a), 89-100.

Castro, J., Henkensiefken, R., Bentz, D., Nantung, T. and Weiss, J., "LWA absorption and desorption: the influence on transport properties of internally cured mortars", Sixth International Conference on Concrete under Severe Conditions, Environment and Loading. CONSEC 10, pp 1543-1550, June 7-9, Merida, Yucatan, Mexico (2010b).

Cerny, R. (2009). "Time-domain reflectometry method and its application for measuring moisture content in porous materials: A review." Measurement 42: 329-336. 
Chatterji S. (1976). "Drying shrinkage of cement paste and concrete: a reappraisal of the measurement technique and its significance. Cement Concrete Res 1976; 6(1): 145-148.

Christensen, B. J., R. T. Coverdale, R. A. Olson, S. J. Ford, E. J. Garboczi, H. M. Jennings, and T. O. Mason. "Impedance Spectroscopy of Hydrating Cement-Eased Materials - Measurement, Interpretation, and Application." Journal of the American Ceramic Society 77, no. 11 (1994): 2789-804.

Coates, K., S. Mohtar, B. Tao, and J. Weiss (2009). Can Soy Methyl Esters Reduce Fluid Transport and Improve the Durability of Concrete? Transportation Research Board, Volume 2113, pp. 22-30

Conde, M. (2004). Aqueous Solutions of Lithium and Calcium Chlorides: Property Formulations for Use in Air Conditioning Equipment Design. International Journal of Material Sciences, Vol. 43, No. 4, pp 367-382.

Crank, J., The Mathematics of Diffusion, 2nd ed. New York: Oxford University Press, 1980.

DeSouza SJ, Hooton RD, Bickley JA. Evaluation of laboratory drying procedures relevant to field conditions for concrete sorptivity measurements. Cem Concrete Agg 1997; 19(2): 59-63

DeSouza SJ, Hooton RD, Bickley JA. A field test for evaluating high performance concrete covercrete quality. Can J Civil Eng 1998; 25(3): 551-556.

Dhir, R. K., Hewlett, P. C. and Chan, Y. N. (1989). "Near Surface Characterizatics of Concrete: Intrinsic Permeability." Magazine of Concrete Research 41(147): 87-97.

Dullien, F. (1979), "Porous Media: Fluid Transport and Pore Structure”, Academic Press, New York.

Elsharief, A., Cohen, M. D. and Olek, J. (2005). "Influence of lightweight aggregate on the microstructure and durability of mortar." Cement and Concrete Research 35(7): 1368.

Fagerlund, G., "Modeling the Service Life of Concrete Exposed to Frost," International Conference on Ion and Mass Transport in Cement-Based Materials, University of Toronto, October 1999

Fagerlund G. Predicting the service-life of concrete exposed to frost action through a modeling of the water absorption process in the air pore system. The Modeling of Microstructure and its Potential for Studying transport Properties and Durability, Jennings H, Kropp J, Scrivener K, editors. The Netherlands, 1996; p. 503-539. 
1 Figg, J. W. (1973). "Methods of measuring the air and water permeability of ocncrete."

2 Magazine of Concrete Research 25(85): 213-219.

3

4
Ford, S. J., T. O. Mason, B. J. Christensen, R. T. Coverdale, H. M. Jennings, and E. J. Garboczi. "Electrode Configurations and Impedance Spectra of Cement Pastes." Journal of Materials Science 30, no. 5 (1995): 1217-24.

Ford, S. J., and T. O. Mason. "Combined Bulk and Interfacial Studies of the Cement/Steel System by Impedance Spectroscopy." Techniques to Assess the Corrosion Activity of Steel Reinforced Concrete Structures 1276 (1996): 146-57.

Garboczi, E. J. (1990). "Permeability, diffusivity, and microstructural parameters: A critical review." Cement and Concrete Research 20(4): 591.

Geiseler, J., Kollo, H. and Lang, E. (1995). "nfluence of blast furnace cements on durability of concrete structures." ACI Materials Journal 92(3): 252-257.

Germann Instruments Inc. (2009). "Germann Gas Permeation Test." http://www.germann.org/Products/Guardian/Guardian.pdf.

Germann Instruments Inc. (2009). "Germann Water Permeability Test (GWT)." http://www.germann.org/Products/GWT/GWT.pdf.

Gowripalan, N., Cabrera, J. G., Cusens, A. R. and Wainwright, P. J. (Feb. 1990). "Effect of Curing on Durability." Concrete International: 47-54.

Greenspan, L. (1977). Humidity Fixed Points of Binary Saturated Aqueous Solutions. Journal of Research of the National Bureau of Standards - A, Physics and Chemistry, Vol. 81A, No. 1, pp. 89-96.

Grube, H. and Lawrence, C. D. (1984). Permeability of concrete to oxigen. RILEM Seminar on the durability of concrete under normal outdoor exposure, Hannover University.

Gu, P., and J. J. Beaudoin. "Dielectric Behaviour of Hardened Cement Paste Systems." Journal of Materials Science Letters 15, no. 2 (1996): 182-84.

Gu, P., P. Xie, J. J. Beaudoin, and R. Brousseau. "Ac Impedance Spectroscopy .1. A New Equivalent-Circuit Model for Hydrated Portland-Cement Paste." Cement and Concrete Research 22, no. 5 (1992): 833-40.

Hall, C. (1994). "Barrier Performance of Concrete: A Review of Fluids Transport Theory." Material and Structures 27: 291-306. 
Hall, C. and Yau, M. H. R. (1987). "Water movement in porous building materials - IX. The water absorption and sorptivity of concretes." Building Environment 22: 77-82.

Hall, C., J. Marchand, G. Gerard, and M. Sosoro (1997). Chapter 2: Transport of fluids in homogenous isotropic cementitious composites. In Penetration and Permeability of Concrete, Ed H. W. Reinhardt, RILEM report 16, pp. 5-79.

Hall, C., and W.D. Hoff (2002). Water-Transport in Brick, Stone and Concrete. Spon Press, London.

Hall C. Water sorptivity of mortars and concretes: a review. Mag Concrete Res 1989; 41(146): 51-61.

Hall C. Anomalous diffusion in unsaturated flow: fact or fiction?, Cement Concrete Res 2007; 37(3): 378-385.

Hanson, A. J., Ottosen, N. S. and Peterson, C. G. (1984). Gas permeability of concrete in situ: theory and practice. ACI SP-82, In-situ Nondestructive Testing of Concrete, Detroit.

Hearn N, Hooton D, Mills R. Pore structure and permeability. Significance of Tests and Properties of Concrete and Concrete-Making Materials, ASTM STP 169C. Klieger P, Lamond J, editors; 1994; 240-262.

Henderson, G. D., Basheer, P. A. M. and Long, A. E. (2004). Pull-off test and permeation tests. Handbook on non-detructive tesing on concrete. V. M. Malhotra and N. J. Carino, CRC Press.

Henkensiefken R, Castro J, Bentz D, Nantung T, Weiss J. Water Absorption in Internally Cured Mortar Made with Water-Filled Lightweight Aggregate. Cement Concrete Res 2009; 39(10): 883-892.

Hong, K., and R.D. Hooton (1999). Effect of cyclic chloride exposure on penetration of concrete cover. Cement and Concrete Research, Vol. 29, No. 9, pp 1379-1386.

Hooton RD, Mesisc T, Beal DL. (1993). Sorptivity testing of concrete as an indicator of concrete durability and curing efficiency. Proceedings of the Third Canadian Symposium on Cement and Concrete, Ottawa, Ontario, 1993; p. 264-275.

Hooton R.D., Geiker M. and Bentz E.C. (2002). "Effects of Curing on Chloride Ingress and Implications on Service Life." ACI Materials Journal 99(2): 201-206.

Hwang C, Young J. Drying shrinkage of portland cement pastes. microcracking during drying. Cement Concrete Res 1984; 14(4): 585-594. 
1 Janusz, A (2010). Investigation of Deicing Chemicals and Their Interactions with

2 Concrete Materials. Master Thesis, Purdue University, West Lafayette, IN.

3

4

Katz, A. and Thompson, A. H. (1986). "Quantitaive prediction of permeability in porous rock." Physical Review B 34(11): 8179-8181.

Kelham, M. (1988). A Water absorption test for concrete. Magazine of Concrete Research, Vol. 40, No. 143, pp. 106-110.

Kollek, J. J. (1986). "The determination of the permeability of concrete to oxygen by the Cembureau method - a recommendation " Materials and Structures 22(3): 225-230.

Lane, S., "Laboratory Comparison of Several Tests for Evaluating the Transport Properties of Concrete", VTRC 06-R38, 2006

Levitte, M. (1971). "The ISAT: A non-destructive test for the durability of concrete." British Journal of NDT July: 106-112.

Lo, T. Y. and Cui, H. Z. (2004). "Effect of porous lightweight aggregate on strength of concrete." Materials Letters 58(6): 916.

Lockington D, Parlange JY, Dux P. Sorptivity and the estimation of water penetration into unsaturated concrete. Mater Struct 1999; 32(5): 342-347.

Lu, B. and Torquato, S. (1992). "Nearest-surface distribution functions for polydispersed particle systems." Physical Review A 45(8): 5530.

Macdonald, J. R., and R. K. William, R.K. (1987), "Impedance Spectroscopy: Emphasizing Solid Materials and Systems”. New York: Wiley-Interscience.

MacInnis, C., and Y. R. Nathawad (1980). The Effect of a Deicing Agent on the Absorption and Permeability of Various Concretes. In ASTM Special Technical Publication STP-691, Proceeding of the First International Conference on Durability of Buildings Materials and Components, Ottawa, Canada, pp 485-496.

Maekawa, K., Chaube, R. and Kishi, T. (1999). Modelling of concrete performance: Hydration, microstructure and mass transport. London, Taylor and Francis.

Maltais Y, Samson E, Marchand J. Predicting the durability of Portland cement systems in aggressive environments - laboratory validation. Cement Concrete Res 2004, 34(9); 1579-1589.

Martys NS. Diffusion in partially saturated porous materials. Mater Struct 1999, 32(8): 991-1004. 
1 Martys N. Ferraris CF. Capillary transport in mortar and concrete. Cement Concrete Res

1997; 27(5): 747-760

Martys, N. S. (1995). Survey of concrete transport properties and their measurments. NISTIR 5592, U.S. Departament of Commerce.

Mastrad Limited (2009). "TORRENT Permability tester." http://www.mastrad.com/torrent.htm.

McCarter, W.J., and Curran, P.N. (1984), "The Electrical Response Characteristics of Setting Cement Paste", Magazine of Concrete Research, Vol. 36, No. 126, 42-29.

McCarter, W.J. and Brousseau, R. (1990), "The A.C. Response of Hardened Cement Paste", Cement and Concrete Research, Vol. 20, No. 6, 891-900.

McLachlan, D.S., Blaszkiewics, M., and Newnham, R.E. (1990), "Electrical Resisitivity of Composites", Journal of the American Ceramic Society, Vol. 73, No. 8, 2187-2203.

Mehta, P. K. (1994). Concrete technology at the crossroads - problems and opportunities. Concrete Technology in Past, Present and Future, ACI SP-144.

Meletiou, C. A., Bloomquist, D. G. and Tia, M. (1993). Method and apparatus for testing water permeability of concrete. 5219388. U. S. Patent.

Meletiou, C. A., Tia, M. and Bloomquist, D. G. (1992). "Development of a field permeability test apparatus and method for concrete." ACI Materials Journal Jan-Feb: 8389.

Nantung, T.E., and Byers M. E., "Portland Cement Concrete Pavement Performance Relative to Permeability", Research Need Statement 27, 2006

Neville, A. M. (1995). Properties of Concrete. New York, N. Y., Longman Scientific \& Technical.

Newton, C. J. and Sykes, J. M. (1988). "A galvanostatic pulse technique for investigation of steel corrosion in concrete." Corrosion Science 28(11): 1051-1074.

Nilsson LO. Hygroscopic moisture in concrete - drying, measurements and related material properties. PhD thesis. Lund University, 1980.

Nokken, M. and Hooton, R. D. (2002). "Dependence of Rate of Absorption on Degree of Saturation of Concrete." Cement and Concrete Research 24(1): 20-24.

Nokken, M. and Hooton, R. D. (2004). Discontinuous capillary porosity in concrete Does it exist? Advanced in Concrete through Science and Engineering, ACBM RILEM, Northwestern University, Chicago. 
Otsuki, N., Sagataki, S. and Nakashita, K. (1992). "Evaluation of AgNO3 solution spray method for measurement of chloride penetration into hardened cementitiuos matrix materials." ACI Materials Journal 89(6): 587-592.

Ozylindrim, C., "Fabricating and Testing Low Permeability Concrete for Transportation Structures," VTRC 99-R6 1998

Parrot LJ. Water absorption in cover concrete. Mater Struct 1992; 25(5): 284-292.

Parrott LJ. Moisture conditioning and transport properties of concrete test specimens. Mater Struct 1994; 27(8): 460-468.

Parrott LJ. Factor influencing relative humidity in concrete. Mag Concrete Res 1991; 43(154): 45-52.

Parrott, L. J. and Hong, C. Z. (1991). "Some factors influencing air permeation measurements in cover concrete." Materials and Structures 24: 403-408.

Pellinen, T., Weiss, W. J., Kuczek, T., and Duksas, G., "Comparison of Various INDOT Testing methods and Procedures to Quanfity Variability in Measured Bituminous and Concrete Properties, “ 2005

Perraton, D. and Aitcin, P.C. (1992). Permeability as seen by the researcher. High Performance Concrete: From Material to Structure, London, E. \& F.N. Spon.

Penko, M. (1983), "Some early Hydration Processes in Cement Pastes as Monitored by Liquid Phase Composition Measurements", Ph.D. Dissertation, Purdue University, West Lafayette, Indiana.

Poursaee, A., Weiss, J. (2010), "An automated electrical monitoring system (AEMS) to assess property development in concrete", Automation in Construction, Vol. 19, No 4, pp 485-490.

Peterson, K.W., R.A. Swartz, L.L. Sutter, T.J. Van Dam (2001). Hardened Concrete Air Void Analysis with a Flatbed Scanner. In Transportation Research Record: Journal of the Transportation Research Board, No. 1775, Transportation Research Board of the National Academies, Washington, D.C., pp. 36-43.

Phang, S. and R.H. Stokes (1980). Density, Viscosity, Conductance and transference numbers of concentrated aqueous magnesium chloride at 25C. Journal of Solution Chemistry, Vol. 9, No. 7, pp. 497-505.

Pour-Ghaz, M., F. Rajabipour, J. Couch, and J. Weiss (2009). Numerical and experimental assessment of unsaturated fluid transport in saw-cut (notched) concrete 
elements. In 'Modeling as a solution to concrete problems', SP-266 American Concrete Institute.

Powers TC, Brownyard TL. Studies of the physical properties of hardened Portland cement paste. American Concrete Institute Proceedings 1946; 43: 469-482.

Powers TC, Copeland LI, Mann HM. Capillary continuity or discontinuity in cement pastes. J. PCA Res. Dev. Lab 1959; 1(2): 38-48.

Proceq (2009). "Torrent permeability tester." http://www.proceq.com/fileadmin/documents/proceq/products/Concrete/Torrent/English/ Proceq_Brochure.

Rajabipour, F. (2006), "In Situ Electrical Sensing and Material Helath Monitoring in Concrete Structures", PhD Dissertation, Purdue University p. 173.

Rajabipour, F., Weiss, W. J., and Abraham, D. M. (2004). "Insitu Electrical Conductivity Measurements to Assess Moisture and Ionic Transport in Concrete", The advances in Concrete through Science and Engineering, a RILEM International Symposium, Evanston, Illinois.

Rajabipour, F., and Weiss, W. J., (2006) "Electrical Conductivity of Drying Cement Paste", RILEM Materials and Structures, Vol. 40, pp. 1143-1160

Sabir B, Wild S, O'Farrell M. A water sorptivity test for mortar and concrete. Mater Struct 1998; 31(8): 568-574.

Samaha H. Hover K. Influence of microcracking on the mass transport properties of concrete. ACI Mater J 1992; 89(4): 416-424.

Sant, G., Rajabipour, F., and Weiss, W.J., (2008) 'tHE Influence of Temperature on Electrical Conductivity Measurements and Maturity Predictions in Cementitious Materials during Hydration', The Indian Concrete Journal, pp. 1-10.

Sant, G., A. Eberhardt, D.P. Bentz, and J. Weiss (2010). Influence of ShrinkageReducing Admixtures on Moisture Absorption in Cementitious Materials at Early Ages. Journal of Materials in Civil Engineering, Vol. 22, No. 3, pp 277-286.

Scherer, G., and G.S. Wheeler (2009). Silicate Consolidants for Stone. Key Engineering Materials, Vol. 391, pp. 1-25.

Schönlin, K. and Hilsdorf, H. (1987). Evaluation of effectiveness of curing concrete structures. Katharine and Bryant Mather Int. Conf. on Concrete Durability, Farmington Hills, MI, ACI SP-100, American Concrete Institute. 
Shane, J. D., Hwang, J.-H., Sohn, D., Mason, T. O. and Jennings, H. M. (1997). "Recent developments in the measurement of transport properties in cement-based materials. in mechanism of chemical degradation of cement-based systems." Emerging transport property measurement techniques.

Shafiq, N. and Cabrera, J. G. (2004). "Effects of initial curing condition on the fluid transport properties in OPC and fly ash blended cement concrete." Cement and Concrete Composites 26: 381-387.

Shi, X., L., Fay, M.M. Peterson, and Z. Yang (2010). Freeze-Thaw damage and chemical change of a Portland cement concrete in the presence of diluted deicers. Materials and Structures, Vol. 43, pp. 933-946.

Sosoro, M. (1998). "Transport of organic fluids through concrete." Material and Structures 31: 162-169.

Spragg R. Castro J. Li W, Pour-Ghaz M, Huang P, Weiss J. Wetting and drying of concrete in the presence of deicing salt solutions. Submitted to Cement and Concrete Composite, 2010.

Stanish, K. D., Hooton, R. D. and Thomas, M. D. A. (1997). Testing the chloride penetration resistance of concrete: a literature review. Prediction of Chloride Penetration in Concrete, FHWA Contract DTFH61-97-R-00022

Sutter, L., K. Peterson, G. Julio-Betancourt, D. Hooton, T. Van Dam, and K. Smith (2008). The deleterious chemical effects of concentrated deicing solutions on Portland cement concrete. South Dakota Department of Transportation, Final Report.

Tang, L. and Nilsson, L. O. (1992). "Chloride Diffusivity in High Strength Concrete." Nordic Concrete Research 11: 162-170.

The Concrete Society (1988). Permeability testing of site concrete - a review of methods and experiences, Technical Report 3. London.

Torquato, S. (2002), "Random Heterogeneous Materials; Microestructure and Macroscopic Properties", Springer - Verlag, New York.

Torrent, R., Basheer, M. and Goncaves, A. F. (2007). Non-destructive methods to measure gas permeability. Non-destructive evaluation of the penetrability and thickness of the concrete cover. R. Torrent and L. F. Luco, RILEM, Report 40.

Torrent, R. J. (1992). "A two-chamber vacuum cell for measuring the coefficient of permeability to air of the concrete cover on site." Materials and Structures 25: 358-365. 
Tremblay M-H, Jolin M., Willem X. and Marchand J, 'La résistance à l'écaillage des bétons contenant des ajouts cimentaires', ACI Seminar progress in concrete field, Boucherville, Québec, Canada, 30 November 2005

Wang, K., Cable, J. K. and Ge, Z. (2006). "Evaluation of Pavement Curing Effectiveness and Curing Effects on Concrete Properties." Journal of Materials in Civil Engineering 18(3): 377-389.

Wang, K., D. Nelsen, W. Nixon (2005). Damaging effects of deicing chemicals on concrete materials. Cement and Concrete Composite, Vol. 28, pp 173-188.

Weiss, W. J. (1999) Prediction of Early-Age Shrinkage Cracking in Concrete. PhD Dissertation, Northwestern University, Evanston, IL.

Weiss (1999). Lecture notes of CE 530 Properties of Concrete. Purdue University, West Lafayette, IN.

Wenner, F. (1916). A Method of Measuring Earth Resistivity. Bulletin of the Bureau of Standards. 12: 469-478.

Whiting D. and Cady P.D. (1992). Method of field measurement of concrete permeability. Condition Evaluation of Concrete Bridges Relative to Reinforcement Corrosion, SHRP-S/FR-92-109, Washington, D.C., National Research Council.

Wiley, G. and Coulson, D. C. (1939). "A simple test for water permeability of concrete." Journal of the American Concrete Institute 34: 65-75.

Winslow, D. and Liu, D. (1990). "The pore structure of paste in concrete." Cement and Concrete Research 20(2): 227.

28

29

Yang, Z, Weiss, W. J., and Olek, J., "Interaction between Micro-Cracking and Reduced Durability of Concrete: Developing Methods for Quantifying the Influence of Cummulative Damage in Life-Cycle Modeling", 2005.

Yang Z, Weiss J, Olek J. Water absorption in partially saturated fracture concrete. RILEM workshop: transport mechanism in cracked concrete. Ghent, 2007.

Yang Z, Weiss J, Olek J. Water transport on concrete damaged by tensile loading and freeze-thaw cycling. J Mater Civil Eng 2006; 18(3): 424-434.

Yang Z. Assessing cumulative damage in concrete and quantifying its influence on life cycle performance modeling. PhD thesis, Purdue University, West Lafayette, Indiana, 2004.

Zhang, M.-H. and Gjørv, O. E. (1990). "Microstructure of the interfacial zone between lightweight aggregate and cement paste." Cement and Concrete Research 20(4): 610. 
1

2 Zia, P., Ahmad, S. and Leming, M. (1994). High-Performance Concretes, A State-of-Art 3 Report FHWA, FHWA-RD-97-030 